\title{
Mikro- und makroskopische Eigenschaften von statistisch und nicht-statistisch aufgebauten Copolymeren
}

\section{Radikalische Polymerisationen in einem weiten Zustandsbereich bis hin zu hohen Drücken und Temperaturen}

\author{
Dissertation \\ zur Erlangung des Doktorgrades \\ der Mathematisch-Naturwissenschaftlichen Fakultäten \\ der Georg-August-Universität zu Göttingen
}

vorgelegt von

Björn Steisel

aus Kassel

Göttingen 2007 
D 7

Referent: Prof. Dr. M. Buback

Korreferent: Prof. Dr. K. Samwer

Tag der mündlichen Prüfung: 01.11.2007 
Wo es an Beratung fehlt, da scheitern die Pläne, wo viele Ratgeber sind, gibt es Erfolg.

Die Bibel, Altes Testament, Sprichwörter 15, 22 



\section{Inhaltsverzeichnis}

1

3

3.1

3.1 .1

3.1 .2

3.1.3

3.1 .4

3.1 .5

3.1 .6

3.1 .7
Zusammenfassung...................................................................... 1

Literaturverzeichnis Kapitel 1............................................... 2

Einleitung............................................................................... 3

Literaturverzeichnis Kapitel 2............................................. 4

Materialien und Experimentelles............................................... 5

Hochdrucksynthese von Polyethylencopolymeren.................... 5

Verwendete Chemikalien.............................................................. 5

Experimentelle Anordnung zur Hochdruck-Copolymerisation.. 5

Auswahl der Messbedingungen.............................................. 13

Durchführung der Polymerisationsexperimente....................... 13

Dosierung des Comonomers (Meth)Acrylsäure....................... 16

Dosierung der Initiatorlösung............................................... 18

Durchführung von Copolymerisationsexperimenten mit

(Meth)Acrylsäure als Comonomer.......................................... 18

3.1.7.1 Durchführung der Copolymerisation........................................ 18

3.1.7.2 Hochdruckproben.............................................................. 19

3.1.7.3 Niederdruckproben.......................................................... 20

3.1.7.4 Initiatorlösung................................................................. 22

3.1.7.5 Verwendung eines Initiatorcocktails...................................... 24

3.1.7.6 Initiatorverbrauch bei Hoch- und Niederdruckproben.............. 24

3.1.7.7 Probenherstellung unter adiabatischen Bedingungen................ 25

3.1.7.8 Hergestellte Polymerproben.................................................... 25

3.2 Experimentelle Methoden zur Ermittlung mikro-struktureller

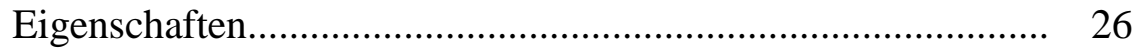

3.2.1 Kalorimetrische Untersuchungen......................................... 26

3.2.2 FT-IR-spektroskopische Untersuchungen.............................. 29

3.2.3 Dynamisch-mechanische Analyse.......................................... 32

3.2.3.1 Aufbau der DMA.............................................................. 32

3.2.3.2 Kalibrierung................................................................... 34

3.2.3.3 Die Messmodi uniaxiale Kompression und Dehnung............... 34 
3.2.3.4 Präparation von zylindrischen Proben..................................... 35

3.2.4 Dielektrische Spektroskopie................................................ 37

3.2.5 Bestimmung der Molmassenverteilung................................ 38

3.2.6 Gepulste Laserdeposition.................................................. 39

3.2.7 Rasterelektronenmikroskopie........................................... 40

3.2.8 $\quad{ }^{1} \mathrm{H}$ - und ${ }^{13} \mathrm{C}-\mathrm{NMR}-$ Spektroskopie in fester Phase.................... 40

3.2.8.1 Aufbau des NMR-Spektometers........................................... 41

3.2.8.2 Bestimmung der Mikrostruktur........................................... 42

3.2.9 Bestimmung der Mikrohärte.................................................. 46

3.2.10 Thermogravimetrische Analyse............................................ 46

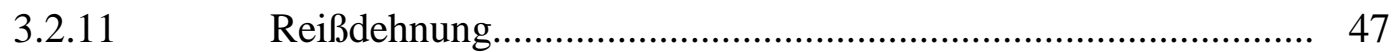

3.2.12 Optische Messungen an Polymeren..................................... 47

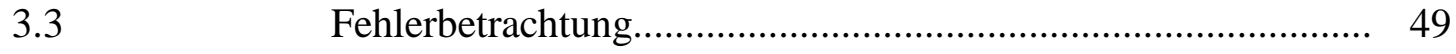

3.3.1 Unsicherheit der Messgeräte.................................................. 49

Literaturverzeichnis Kapitel 3.......................................... 50

Untersuchungen an Ethen-Methacrylsäure-Copolymeren... 53

Bestimmung der Copolymerzusammensetzung....

Bestimmung der Molmassenverteilungen der

Copolymerproben.

Weiterführende ATR-FT-IR-Spektroskopieuntersuchungen..... 68

4.3.1 Vergleich von ATR-FT-IR-Spektren für Hoch- und

Niederdruckproben.

4.3.2 Auswirkung des MAA-Gehalts auf das Verhältnis der

integrierten Absorbanzen Nieder-/Hochdruckprobe

4.3.3 Auswirkung der Synthesetemperatur und des

Monomerumsatzes auf das Verhältnis der integrierten

Absorbanzen Nieder-/Hochdruckprobe.

4.3.4 IR-spektroskopische Untersuchungen an zyklischen

Anhydriden der Ethen-Methacrylsäure-Copolymere................. 74

4.4

Berechnung der Dichte der Reaktionsmischung im Reaktor.... 75

4.1.1 Vergleich der Dichte im Reaktor für Hoch- und

Niederdruckproben....................................................... 75 


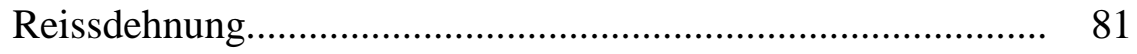

Optische Messungen an Polymeren........................................ 83

Relaxationen im Polymer........................................................ 85

Kurbelwellenbewegungen......

DSC-Analysen an den Homopolymersystemen PE und PMAA und an Copolymerproben EMAA mit verschiedenem

Methacrylsäuregehalt.

Unter adiabatischen Bedingungen hergestellte Copolymere......

Dielektrische Spektroskopie an Copolymeren...

4.13.2 Zweidimensionale NMR-Spektroskopie an EMAA-

Copolymeren zur speziellen Strukturaufklärung der

Niederdruckproben

Ergebnisse der gepulsten Laserdeposition. 
4.14.4 Oberflächenstrukturanalyse von mittels PLD hergestellten PE-Schichten.

4.14.5 Oberflächenstrukturanalyse von mittels PLD hergestellten

EMAA-Schichten............................................................ 140

4.14.6 Molmassenverteilung einer deponierten Copolymerprobe........ 142

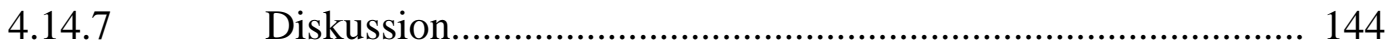

4.15 Vergleich der Systeme Ethen/Methacrylsäure und

Ethen/Methylmethacrylat.................................................... 145

Literaturverzeichnis Kapitel 4.......................................... 151

5

Konzept des "Chemical Confinements" .................................. 153

5.1 Definition und Ursachen des "Chemical Confinements". 153

5.2

Überprüfung der Theorie des "Chemical Confinements"

anhand der erzielten Analyseergebnissen...

5.2.1 DSC-Analysen: Chemical Confinement in Niederdruckproben 157

5.2.2 Chemical Confinement bei der mechanischen Spektroskopie... 160

5.2.3 Chemical Confinement in der dielektrischen Spektroskopie..... 161

Literaturverzeichnis Kapitel 5............................................ 161

6

Ausblick.............................................................................. 163

Literaturverzeichnis Kapitel 6............................................ 164

Anhang......................................................................................................... 165

7.1

Abkürzungsverzeichnis..................................................... 165

7.2

TÜV-Überprüfung der Ethenreinigungstürme........................ 168

7.2.1 Allgemeine Vorbereitungen................................................ 168

7.2.2 Deaktivierung des Katalysators............................................ 168

7.2.3 Abbau der Türme........................................................... 170

7.2.4 Wiederbefüllung und Reinstallation der Reinigungstürme........ 171

7.2.5 Reduktion des Katalysators............................................... 171

7.2.6 Regenerierung des Molsiebs............................................... 172

7.3 Überblick über maßgebliche Parameter der synthetisierten

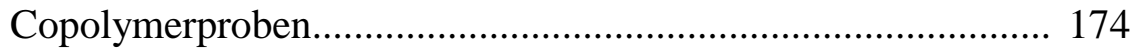




\section{Zusammenfassung}

Die Verknüpfung von mikroskopischen und makroskopischen Eigenschaften stellt seit jeher ein interessantes Themengebiet dar. Die vorliegende Arbeit beschäftigt sich mit der Synthese von Ethen-Methacrylsäurecopolymeren im Bereich der Phasengrenze, wo ein nicht-statistischer Einbau der Methacrylsäuregruppen nachgewiesen wurde ${ }^{[1,2]}$ und mit der detaillierten Untersuchung der anwendungsrelevanten Eigenschaften. Als direkte Referenz zu diesen Proben wurden Copolymere in erheblichem Abstand zur Phasengrenze unter Beibehaltung möglichst vieler Syntheseparameter hergestellt, bei denen eine statistische Verteilung der Säuregruppen im Copolymer vorliegt. Dieser signifikante Unterschied konnte mittels zweidimensionaler NMR-Spektroskopie aufgezeigt werden. Die bereits unmittelbar nach der Synthese visuell festzustellenden Unterschiede in der Erscheinungsform der bei verschiedenen Drücken synthetisierten Proben wurden mittels zahlreicher Analyseverfahren quantifiziert. Härtemessungen und Reissfestigkeitsuntersuchungen bescheinigten den statistisch aufgebauten Proben eine höhere mechanische Festigkeit als den nicht-statistisch aufgebauten. Optische Messungen zeigten für diese Proben eine geringere Klarheit und höhere Trübung im Vergleich zu den nicht-statistisch aufgebauten Proben auf.

Die signifikanten Unterschiede der Hoch- und Niederdruckproben (weit entfernt und nahe der Phasengrenze synthetisiert) wurden in dieser Arbeit erstmalig in einer zusätzlichen Relaxation beobachtet. So zeigten sich bei kalorimetrischen Untersuchungen für die Niederdruckproben zusätzliche Minima im Wärmefluss bei tieferen Temperaturen als der Glasübergang. Diese Relaxation beruht auf einer zusätzlichen Segmentbewegung, die für Hochdruckproben nicht beobachtet werden konnte. Die dynamisch mechanische Analyse (DMA) zeigte analoge Tendenzen auf. Für die Niederdruckproben ergaben sich hier ebenfalls zusätzliche Signale. Dieser Übergang ergab sich wiederum bei tieferen Temperaturen als der beobachtete Glasübergang der Copolymere. Aufgrund dieser aussagekräftigen Analysen konnte ein theoretisches Modell für eine chemische Behinderung der Segmentbewegung aufgestellt werden. Dieses „Chemical Confinement“ wurde anhand der hergestellten Ethen-Methacrylsäurecopolymere definiert und kann in späteren Arbeiten auf ähnliche Comonomere, wie zum Beispiel Acrylsäure, angewendet werden. In den DMA-Messungen konnte eine erhebliche Temperaturabhängigkeit der 
zusätzlichen Relaxation der Niederdruckproben von den verwendeten Kräften beobachtet werden. Dieser Effekt wurde ausführlich durch Mehrfachvariation der Kräfte aufgezeigt.

Eine weitere Analysemethode für nicht leitende Materialien stellt die dielektrische Spektroskopie (DES) dar. Hier wurden ebenfalls für die Niederdruckproben zusätzliche Relaxationen gefunden. Die signifikanten Unterschiede, die Hoch- und Niederdruckproben aufgrund ihres unterschiedlichen strukturellen Aufbaus aufweisen, konnten in dieser Arbeit anhand einer Vielzahl unabhängiger Analysemethoden gezeigt werden. Die Unterschiede der makroskopischen Eigenschaften können auf den nachweislich unterschiedlichen Aufbau zurückgeführt werden.

Keine Unterscheidungsmöglichkeit für statistisch und nicht-statistisch aufgebaute Copolymere aber dennoch hoch interessante Ergebnisse lieferten Untersuchungen an mittels gepulster Laserdeposition hergestellten dünnen Schichten. Es wurden signifikante Unterschiede für aus polaren und aus unpolaren Monomeren bestehende Polymere herausgearbeitet und diese Erkenntnisse auf die hier hergestellten Copolymere übertragen.

\section{Literaturverzeichnis Kapitel 1}

[1] United States Patent 4,248,990

[2] European Patent 0146620B1 


\section{Einleitung}

Wie so oft begann auch die Geschichte der Polymere durch einen Zufall. Bereits im Jahre 1838 setzte der Franzose Henri Victor Regnault das von ihm entdeckte Vinylchlorid Sonnenlicht aus und erhielt Polyvinylchlorid. Da die Tragweite dieser Reaktion noch nicht richtig eingeschätzt wurde, bestimmten zunächst die aus der Natur gewonnenen polymeren Stoffe das 19. Jahrhundert. Charles Nelson Goodyear stellte 1851 ein Verfahren vor, welches auf der Vermengung von Schwefel mit dem weißen Milchsaft des Kautschukbaumes beruht und zu einem elastischen Gummi führt. ${ }^{[1]}$

Die synthetische Herstellung von Polymeren, basierend auf Erdölprodukten, begann mit Baekeland im Jahr 1907. Das erste Verfahren zu Hochdrucksynthese von Polyethylen wurde 1939 durch die Firma ISI in Großbritannien angewendet. Seit der großindustriellen Herstellung der Polymere haben diese Einzug in das alltägliche Leben gehalten. Dort sind Polymere heutzutage in allen Anwendungsbereichen $\mathrm{zu}$ finden und ersetzen beziehungsweise ergänzen eine Vielzahl traditioneller Werkstoffe wie zum Beispiel Holz, Metall, Glas und Naturprodukte.

Polyethylen ist einer der wichtigsten Kunststoffe. Die Verbesserung der anwendungstechnischen Eigenschaften ist weiterhin Gegenstand der Forschung. Zu einer erheblichen Erweiterung der Anwendungsgebiete hat die Copolymerisation von Ethen geführt. Die in dieser Arbeit untersuchten Ethen-(Meth)Acrylsäure-Copolymere besitzen hervorragende Haftfähigkeit auf Metallen, Papier, Glas und Kunststoffen. Zu Filmen verarbeit sind sie stark formbar und belastbar. Es besteht eine Resistenz gegen Säuren, Basen und organische Lösungsmittel.

Aufgrund dieser hervorragenden Produkteigenschaften werden diese Copolymere großindustriell hergestellt. Ein Studium über die Copolymerisation bei einem Reaktionsdruck von 2000 bar findet sich bei Wittkowski. ${ }^{[2]}$ In der vorliegenden Arbeit soll der Einfluss der Phasengrenze auf die Produkteigenschaften untersucht werden. Aus der Patentliteratur ist bekannt, dass sich Proben, die in einem weiten Abstand von der Phasengrenze, also tief im homogenen Bereich synthetisiert sind, andere Eigenschaften aufweisen, als Proben, welche nahe der Phasengrenze, aber noch im homogenen Bereich, hergestellt werden. ${ }^{[3]}$ Es werden Proben bei maximalem Druck des in Kapitel 3 beschriebenen Minitechnikums hergestellt. Diese Proben werden als Hochdruckproben bezeichnet. Die Niederdruckproben werden bei gleichen Bedingungen, nur bei minimalst 
möglichem Synthesedruck hergestellt, so dass eine Einphasigkeit der Reaktion weiterhin gegeben ist. Für die Hochdruckproben wird eine statistische Anordnung der Monomerbausteine im Polymer erwartet. Die Einbauhäufigkeit der Säuregruppen wird durch die bekannten Copolymerisationsparameter bestimmt. ${ }^{[2]}$ Für die Niederdruckproben wird eine nicht-statistische Anordnung der Säuregruppen erwartet. Der Patentliteratur zufolge sollten hier konjugierte Säuregruppen vorliegen. ${ }^{[3]}$ Das kann zu eingebauten Säuredimeren oder $\mathrm{zu}$ höheren Säureoligomeren im Copolymer führen. Diese nicht-statistische Dimerbildung führt aufgrund der erhöhten Möglichkeit zur Ausbildung von Wasserstoffbrückenbindungen zu einer Veränderung der Materialeigenschaften. Die Unterschiede zwischen Hoch- und Niederdruckproben werden mit einer Vielzahl von analytischen Methoden untersucht. Als hervorragende Möglichkeiten sind hier die DSC („Differential Scanning Calorimetry“) und die DMA (dynamisch-mechanische Analyse) zur Untersuchung von zusätzlichen Phasenübergängen (Relaxationen) zu nennen. Darüber hinaus wurden weitere Analysen ausgeführt, die in Kapitel 3 detailliert erläutert sind. Es wurden häufig signifikante Unterschiede in den unter verschiedenen Bedingungen hergestellten Copolymerproben gefunden (Kapitel 4).

\section{Literaturverzeichnis Kapitel 2}

[1] http://www.seilnacht.com/Lexikon/k_gesch.html

[2] L. Wittkowski, Dissertation, Göttingen (1998)

[3] United States Patent 4,248,990 


\section{$3 \quad$ Materialien und Experimentelles}

\subsection{Hochdrucksynthese von Polyethylencopolymeren}

\subsubsection{Verwendete Chemikalien}

Die zur Polymersynthese verwendeten Chemikalien sind mit dem vom Hersteller angegebenen Reinheitsgrad in Tabelle 3.1 aufgeführt. Ethen wurde innerhalb der Anlage zur Copolymersynthese weiter aufgereinigt (vgl. Kapitel 3.1.2). (Meth)Acrylsäure und die Initiatorlösung wurden unmittelbar vor der Polymerisation mittels eines Degassers (ERC3415 Degasser, ERC, Altengolfsheim (Regensburg)) entgast (vgl. Abbildung 3.1). Die übrigen Chemikalien wurden ohne weitere Reinigung eingesetzt.

\begin{tabular}{ccc}
\hline \hline Chemikalie & Reinheitsgrad & Hersteller \\
\hline \hline Ethen & $99.9 \%$ & Linde \\
Methacrylsäure & $\geq 99 \%$ & Merck-Schuchardt \\
Acrylsäure & $99.5 \%$ & Acros Organics \\
Cyclohexan & $\geq 99 \%$ & Fluka \\
Di-tert-butylperoxid & $\geq 98 \%$ & Merck-Schuchardt \\
tert-Butylperoxyacetat & $50 \%$ in iso- & Akzo Nobel \\
& Dodecan &
\end{tabular}

\begin{tabular}{lllll} 
& Stickstoff & \multicolumn{2}{c}{4.6} & Linde \\
\hline Tabelle 3.1 & $\begin{array}{l}\text { Verwendete } \\
\text { Reinheitsgrad. }\end{array}$ & Chemikalien & mit dem vom & Hersteller angegebenen
\end{tabular}

\subsubsection{Experimentelle Anordnung zur Hochdruck-Copolymerisation}

Die im Rahmen der vorliegenden Arbeit durchgeführten Polymerisationsexperimente wurden bei kontinuierlicher Reaktionsführung mit einer von Busch ${ }^{[1]}$ entwickelten und auch in Arbeiten von Buback et al. ${ }^{[2,3]}$, Lovis ${ }^{[4]}$ und Wittkowski ${ }^{[5]}$ genutzten und beschriebenen Mini-Technikumsanlage zur radikalischen Hochdruck-Polymerisation 
ausgeführt. In diesem Kapitel wird eine Übersicht des Aufbaus des Mini-Technikums gegeben und es werden einzelne Bauteile näher erläutert.

Um eine gleichbleibend hochreine Qualität des eingesetzten Ethens sicherzustellen, wird das Gas innerhalb der Anlage aufgereinigt. Hierzu sind in das Strömungssystem unmittelbar vor der ersten Kompressionsstufe zwei zylindrische Druckbehälter mit einem Volumen von jeweils 11.71 eingebaut. Diese Autoklaven, bestehend aus je einem Edelstahlrohr von $1000 \mathrm{~mm}$ Länge, sind bis zu einem Druck von 50 bar vom TÜVzugelassenen und werden in Abständen von 5 Jahren einer Prüfung unterzogen (siehe Kapitel 7.2). Die Rohre haben einen Außendurchmesser von $141.3 \mathrm{~mm}$ und sind mit aufgeschraubten Flanschen $(200$ x $30 \mathrm{~mm})$ versehen. Die Wandstärke der Behälter beträgt $9.5 \mathrm{~mm}$. Die stirnseitigen Deckel (200 x $30 \mathrm{~mm})$ sind mit den Behältern über jeweils 12 Bolzen (M 12) verschraubt, wobei zur Dichtung O-Ringe aus Viton ${ }^{\circledR}$ verwendet werden. Um den Austrag der Gasreinigungsschüttungen $\mathrm{zu}$ verhindern, sind vor den Auslassbohrungen am Boden der Behälter Sinterfilter angebracht. Der zuerst von Ethen durchströmte Autoklav ist mit einem Katalysator auf Kupfer/Kupferoxidbasis gefüllt (BASF, R3-15), an dem Sauerstoffspuren adsorbiert werden. Im zweiten, mit Molsieb (BASF, EPG 3A) beschickten Druckbehälter werden Wasserspuren entfernt. Der Katalysator muss vor der eigentlichen Verwendung als auch nach erfolgter Sättigung bei einer Temperatur von 200 bis $250{ }^{\circ} \mathrm{C}$ mit Wasserstoff reduziert werden. Das Molsieb wird anschließend bei möglichst hohen Temperaturen (ca. $200{ }^{\circ} \mathrm{C}$ ) und unter reduziertem Druck getrocknet. Für die Einstellung der zur Aktivierung der Schüttungen benötigten Temperatur sind die beiden Behälter jeweils mit einem in einer Messingmatrix eingelassenen Widerstandsheizleiter ausgerüstet. Eine detaillierte Vorschrift zur Deaktivierung und Aktivierung befindet sich in Kapitel 7.2.

In Abbildung 3.1 ist der Gesamtplan des Minitechnikums schematisch dargestellt. Kommerzielles Ethen der Reinheit 3.0 aus einer Druckgasflaschenbatterie (F1-F5) wird durch ein auf $28^{\circ} \mathrm{C}$ geheiztes Reduzierventil von Flaschendruck auf 13 bis 15 bar entspannt. Aufgrund von Sicherheitsaspekten werden lediglich 3 Gasflaschen vorgehalten. Nach Durchströmen der Aufreinigungseinheit (siehe oben) hält ein nachgeschalteter Hochdrucksinterfilter (Swagelock 10-TF, Porenweite $15 \mu \mathrm{m}$ ) eventuelle Staubreste aus der Katalysator- bzw. Molsiebschüttung zurück. 

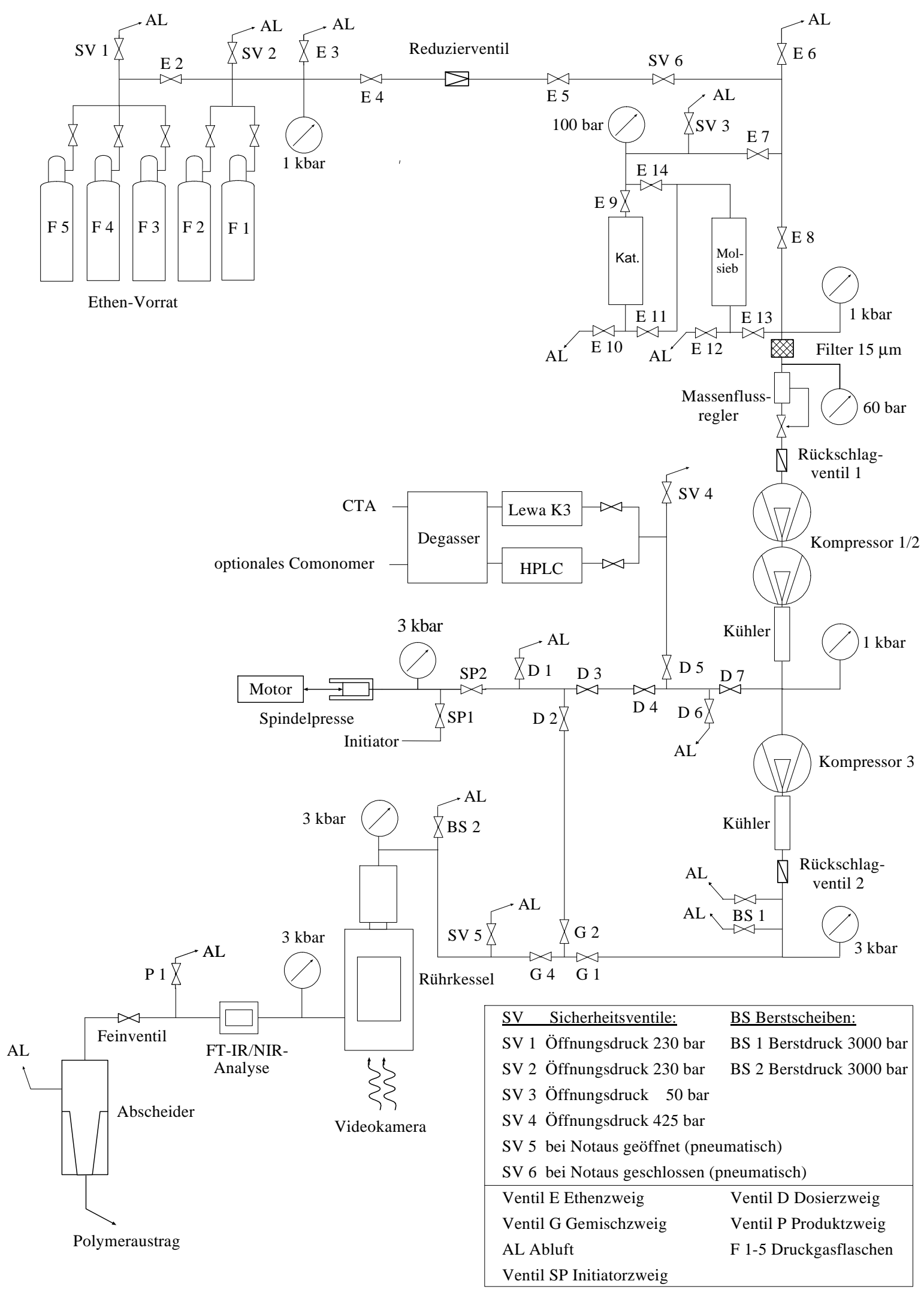

Abbildung 3.1 Schematische Darstellung der Anlage zur Hochdruck-Polymerisation. ${ }^{[7]}$ 
Das nunmehr hochreine Ethen $\left(\mathrm{O}_{2}<0.1 \mathrm{ppm}\right.$, nach Angabe des Katalysatorherstellers) strömt durch einen Massenflussregler (5851E, Brooks-Instrument, Mannheim), welcher den Massenfluss misst und entsprechend einer externen Sollwertvorgabe regelt. Anschließend wird das Ethen von einem zweistufigen Membrankompressor (Hofer, Mühlheim / Ruhr) auf ca. 250 bar verdichtet, abhängig vom Endreaktionsdruck. Durch einen einstufigen Nachverdichter (Hofer, Mühlheim / Ruhr) wird der gewünschte Druck von bis zu 2500 bar erreicht. Vor dieser dritten Kompressionsstufe können mittels einer computergesteuerten Kolbenmembranpumpe (Typ K3, Lewa) und einer HPLC-Pumpe (Typ Maxi-Star 1000, Knauer) Flüssigkeiten in die Saugseite des Nachverdichters gefördert werden, wobei 10 vol\% nicht überschritten werden dürfen. Das Comonomer, Methacrylsäure oder Acrylsäure, wird mittels der Kolbenmembranpumpe gefördert, da eine erhöhte Festigkeit gegen korrodierende Substanzen besteht. Die Initiatorlösung, bestehend aus den unter Tabelle 3.1 angegeben Initiatoren und Cyclohexan, wird mittels der HPLC-Pumpe ebenfalls vor der dritten Verdichtungsstufe eingeleitet. Eine eventuell auftretende vorzeitige Polymerisation, die sich in Verstopfung des Kompressors und der Kapillaren geäußert hätte, wurde nicht beobachtet. Zum Entfernen der in den Initiatorlösungen und im Comonomer gelösten Gase, wird der Saugstrom der Dosierpumpen durch einen Degasser (siehe Kapitel 3.1.1) geleitet.

Alternativ kann der Initiator auch mittels einer durch Schrittmotoren gesteuerten Spindelpresse ${ }^{[6]}$ sowohl vor der dritten Kompressorstufe bei etwa 250 bar als auch nach derselben bei dem gewählten Reaktionsdruck eindosiert werden. Bei dieser Copolymerisation im Vergleich zur Homopolymerisation ${ }^{[7]}$ von Ethen wird eine erhebliche Menge Initiator benötigt. Um den Einsatz der Spindelpresse versuchstechnisch effizient zu gestalten, würde somit eine hohe Konzentration des Initiators benötigt. Durch hohe Druckschwankungen könnte eine erhebliche Menge Initiator über ein kleines Zeitintervall in den Reaktor gelangen, was zu einer Zersetzung der Reaktionsmischung führen kann. Auf die Eindosierung mittels Spindelpresse wurde in dieser Arbeit verzichtet. Die so hergestellte Mischung gelangt anschließend über 1/4 Zoll-Hochdruckkapillaren in den Hochdruck-Hochtemperatur-Rührautoklaven, in dem die Polymerisation stattfindet. Für die Eindosierung der Reaktionsmischung in den Rührkessel gibt es zwei Möglichkeiten. Zum einen kann die Dosierung seitlich erfolgen. Dies hat zum Vorteil, dass eine frühstmögliche Durchmischung der Reaktanden gewährleistet ist. Die zweite Variante ist die Einspeisung durch den Rührantrieb selbst. ${ }^{[1,5,6]}$ Hierdurch wird vermieden, dass bei Druckschwankungen Reaktionsprodukte in den Innenraum des Rührantriebs eingespült 
werden und sich dort ablagern. Des Weiteren wird der Totraum, also der Raum des Reaktors, in dem kein ständiger Fluss neuer Reaktanden stattfindet, minimiert. Die Homogenität der Reaktionsmischung kann durch ein auf der Unterseite des Reaktors eingelassenes Saphirfenster visuell über eine Videokamera mit angeschlossenem Monitor kontrolliert werden. Optional kann die Reaktionsmischung nach Verlassen des Rührkessels durch eine optische Hochdruckzelle ${ }^{[8]}$, die im Strahlengang eines FT-IR/NIRSpektrometers (IFS 88, Bruker) fixiert ist, geleitet werden.

Durch anschließendes Entspannen der Reaktionsmischung an einem speziellen Feinventil ${ }^{[2]}$ wird das Polymer in einem Normaldruckabscheider ${ }^{[5]}$ von den gasförmigen Bestandteilen separiert und quantitativ in mit Schlitzen zur Gasableitung versehenen Schraubdeckelflaschen aus Polyethylen aufgefangen.

Die Temperaturen im Reaktor, in der optischen Hochdruckzelle, an den Kapillaren und Ventilen, werden mit Mantelthermoelementen (Nickel-Chrom gegen Nickel-ChromAlumel, CIA S250, CGE Alsthom) gemessen. Im Reaktor sind dazu an drei Positionen Messstellen vorgesehen (siehe unten), so dass eventuell auftretende Temperaturgradienten leicht erkannt werden können. Der Reaktor, die Feinventile und die IR-Hochdruckzelle werden elektrisch mit Mantelheizleitern (Pyrolon-M. $16 \Omega \mathrm{m}^{-1}$, Les Cables de Lyon) beheizt, die in jeweils über die Bauteile geschobenen Messingmatrizen eingelassen sind. Für die Temperaturregelkreise werden PID-Regler (Eurotherm, Typ 19, Typ 815 und Typ 2116) eingesetzt. Im Hochdruckteil der Anlage werden die Drücke mit DMS-DruckUmwandlern (HBM-Messtechnik, Klasse 0.2, Maximaldruck $3 \mathrm{kbar}$ ) an folgenden Positionen gemessen: nach dem Nachverdichter, am Eingang des Rührantriebs und optional vor der IR-Zelle.

Um mögliche Gefahren durch versagende Hochdruckbauteile abzuwenden, sind alle Anlagekomponenten mit Überdruckventilen bzw. Berstscheiben ausgestattet (siehe Abbildung 3.1). Darüber hinaus sind in der Druckschutzkabine und außerhalb Notausschalter angebracht, mit denen über elektropneumatisch gesteuerte Ventile gleichzeitig die Ethenversorgung unterbrochen und die Anlage in die Abluft druckentlastet werden kann.

Um sicherzustellen, dass jederzeit ein ausreichender Abluftstrom vorhanden ist wurden elektrische Überwachungseinheiten installiert (ifm electronic, SN 0150). Um ein nicht zündfähiges Gemisch zu erhalten, wird ein Abluftstrom von mindestens $4 \mathrm{~m} \cdot \mathrm{s}^{-1}$ bei einem Förderstrom von $1 \mathrm{~kg} \cdot \mathrm{h}^{-1}$ Ethen eingehalten. Das entstehende Ethen-Luftgemisch liegt somit als zu mageres Gemisch für eine Durchzündung vor. Beim Unterschreiten des 
Grenzwertes ist das Experiment unter Einhaltung der notwendigen Sorgfalt umgehend zu beenden und die Fehlerursache zu beheben.

\section{Hochdruck-Rührkessel}

Der Hochdruck-Rührkessel stellt das Kernstück der Minitechnikumsanlage dar. Im Folgenden werden die wesentlichen Baugruppen beschrieben. Detaillierte Informationen über die Konstruktion und Entwicklung des Autoklaven finden sich bei Busch ${ }^{[1]}$, Buback et al. ${ }^{[2]}$ und Lovis ${ }^{[4]}$. In Abbildung 3.2 ist der Hochdruck-Hochtemperatur-Autoklav dargestellt. Der Autoklav besitzt unter Berücksichtigung aller Einbauten ein Innenvolumen von ca. $50 \mathrm{~cm}^{3}$. Er besteht aus einem zylindrischen Hohlkörper von $170 \mathrm{~mm}$ Länge und besitzt einen Außendurchmesser von 150 mm. Der Innendurchmesser beträgt $42 \mathrm{~mm}$.

Der Körper des Autoklaven sowie der obere und untere Flansch sind aus einer hochwarmfesten Nickelbasislegierung (RGT 601, Werkstoff-Nr. 2.4668, Arbed-Saarstahl) gefertigt. Das Verhältnis von Innen- zu Außendurchmesser erlaubt unter Berücksichtigung eines Sicherheitsbeiwertes von 2.5 in Verbindung mit dem gewählten Material eine maximale Druckbelastung von 3000 bar bei $300{ }^{\circ} \mathrm{C}$.

Die Unterseite des Reaktors wird durch einen Stempel (RGT 12, Werkstoff-Nr. 2.4969, Arbed-Saarstahl) mittels einer Metall-Metall-Konusdichtung verschlossen. Der Stempel wird durch den unteren Flansch mit zwölf Dehnbolzen mit einem Drehmoment von jeweils $90 \mathrm{Nm}$ auf den Autoklaven-Hauptkörper gepresst. Der Einsatz von Dehnbolzen verhindert, dass die Gewindebohrungen im Reaktorblock bei hohen Drucksprüngen beschädigt werden. Der untere Dichtstempel besitzt eine zentrale Bohrung von $21.2 \mathrm{~mm}$ Durchmesser. Dieser Stempel trägt zur optischen Kontrolle des Reaktorinnenraums ein Fenster aus synthetischem Saphir (UV-Grade, Roditi, Union Carbide). Der Durchmesser des Saphirfensters beträgt $38.1 \mathrm{~mm}$, bei einer Höhe von $22.4 \mathrm{~mm}$. Auf der Seite des Rührantriebs wird der Reaktor durch eine Dichtlinse (Werkstoff RGT 12) ebenfalls über Konusdichtungen abgeschlossen. Die Linse ist axial durchbohrt, um den Rührkäfig über eine Welle mit dem Rührantrieb zu verbinden. Der Innendurchmesser der Linse beträgt $11 \mathrm{~mm}$. An der Unterseite der Linse befindet sich ein Edelstahlkugellager (SS-6000-2 ZJ), von dem zum besseren Durchgang des Eduktstromes die seitlichen Dichtungsscheiben (soweit vorhanden) entfernt sind. Das Lager wird mit einer Überwurfmutter in der Dichtlinse mit Linksgewinde fixiert.

Der Antrieb des Rührers erfolgt über eine Hochdruck-Magnetkupplung (Typ 0,75-4-50 KMP 35N Spec., Autoclave Engineers). Um einen Wärmefluss vom Reaktor zur 
Magnetkupplung zu minimieren, ist der Verbindungsflansch wassergekühlt. Die äußeren Permanentmagnete werden über eine Riemenscheibe von einem Syncro-Servo-Motor (Seidel) mit entsprechender Regelelektronik angetrieben. Die bis zu $1500 \mathrm{~min}^{-1}$ stufenlos einstellbaren Drehzahlen des Rührantriebs werden über eine Hallsonde kontrolliert. Als Rührkörper wurde bei den Polymersynthesen der von Lovis ${ }^{[4]}$ entwickelte KäfigPropeller-Rührer verwendet. Anhand von Messungen der Verweilzeitverteilung wurde belegt, dass der Reaktor mit dem verwendeten Rührertyp und dem beschriebenen Dosierweg als annähernd ideal vermischter Rührkesselreaktor beschrieben werden kann. ${ }^{[9]}$ Der Rührkessel besitzt 6 Bohrungen mit einem Durchmesser von jeweils $1.8 \mathrm{~mm}$, die im Winkel von $90^{\circ}$ in drei unterschiedlichen Höhen radial eingelassen sind. In jeweils einer der oberen und unteren Bohrungen wird die Temperatur der Reaktorwand gemessen. Die beiden mittleren Bohrungen sind so angeordnet, dass sie sich gerade über dem Saphirfenster, also direkt oberhalb des Reaktorbodens, befinden. Eine der Bohrungen dient als Auslass der Reaktionsmischung, die andere wird zur Einführung eines Mantelthermoelements genutzt, mit welchem die Temperatur der Reaktionsmischung in der Mitte des Reaktorinneren gemessen werden kann. Ein weiteres Thermoelement schließt in einer der oberen Bohrungen passgenau mit der Reaktorwand ab. Hier wird ein weiterer Messwert für die Innentemperatur des Reaktors erhalten. Zur Vermeidung einer Umspülung des Thermoelements mit der Reaktionsmischung wurde ein exakt in die Bohrung passender Durchmesser des Thermoelements gewählt. 


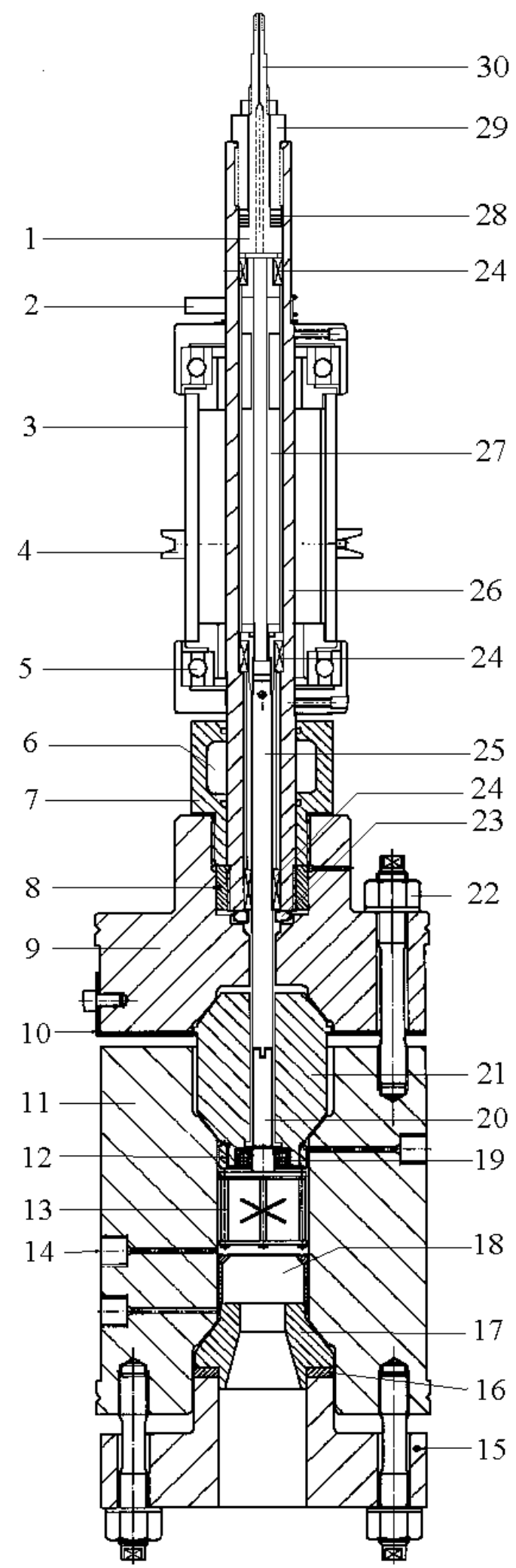

1 Deckel Bridgman-Verschluss

2 Hall-Sonde

3 Gehäuse Rührantrieb

4 Riemenscheibe

5 Kugellager Rührantrieb

6 Wasserkühlung

7 Andruckmutter

8 Kontermutter

9 oberer Flansch

10 Halteblech für Dichtlinse

11 Autoklavenkörper

12 Überwurfmutter

13 Rührer

14 Auslass bzw. Bohrung Thermoelement 15 unterer Flansch

16 Gleitring Rührerlager

17 Stempel

18 Saphirfenster

19 Lager für Antriebswelle

20 Antriebswelle

21 Dichtlinse I

22 Mutter

23 Dichtlinse II

24 Graphitlager Rührwelle

25 Rührwelle

26 Druckmantel Rührantrieb

27 Magnetwelle

28 Bridgeman-Packungen

29 Konterschraube Bridgman-Verschluss 30 Einlasskapillare

Abbildung 3.2: Hochdruck-Rührkessel ${ }^{\text {17] }}$ 


\subsubsection{Auswahl der Messbedingungen}

Der experimentell zugängliche Druck- und Temperaturbereich ist für die Copolymerisation von Ethen mit (Meth)Acrylsäure aufgrund folgender Aspekte eingeschränkt:

\section{(1) Apparative Druck- und Temperaturgrenzen:}

Der für die Synthesen eingesetzte Hochdruckautoklav ist inklusive der Anbauteile bis zu maximalen Reaktionsdrücken von 3000 bar und Temperaturen von $300{ }^{\circ} \mathrm{C}$ einsetzbar.

\section{(2) Phasenverhalten:}

Der zugängliche Druck- und Temperaturbereich wird zu geringen $p$ - und $T$-Werten durch die Inhomogenität der Reaktionsmischung begrenzt. Während der Synthese sollte Mehrphasigkeit vermieden werden, da ansonsten die Reaktionsführung durch verstärktes Reaktorfouling erschwert und das Risiko einer thermischen Zersetzungsreaktion des Ethens erhöht wird. Zudem kann nicht mehr garantiert werden, dass es sich um Copolymere handelt, die unter chemisch wohldefinierten und einheitlichen Bedingungen hergestellt wurden. In dieser Arbeit wurden die Copolymere hinsichtlich ihrer statistischen Abfolge von Monomerbausteinen in Copolymer betrachtet. In mehrphasigen Systemen liegt gerade bei dieser Art von Copolymerisation, wo ein polares und ein unpolares Monomer benutzt wurde, eine Anhäufung der Monomere in einzelnen Phasen vor, was zu einer erheblichen Veränderung der Monomerbausteinabfolge führt.

Das Phasenverhalten für die Systeme Ethen/Poly(Ethen-co-(Meth)Acrylsäure) ist literaturbekannt. ${ }^{[10]}$ Aufgrund eines eventuellen Cosolvenseffektes des Comonomers und der Umsatzabhängigkeit des „cloud point pressure“, wo sich erste ausfallende Wolken des Polymers bilden, dient das literaturbekannte Phasendiagramm als Richtwert für die Synthesebedingungen.

\subsubsection{Durchführung der Polymerisationsexperimente}

\section{Vorbereitung eines Syntheseexperiments}

Vor jedem Experiment werden der Reaktor und alle nachfolgenden Komponenten der Anlage zerlegt und gründlich gereinigt. Hierzu werden eventuelle Polymeranhaftungen nach Aufweichen bei Temperaturen bis $180^{\circ} \mathrm{C}$ (in flüssigem Isododekan) mechanisch entfernt und Lösungsmittelreste mit Aceton abgespült. Polymerreste in zugesetzten 
Hochdruckbauteilen und Kapillaren werden auf bis $\mathrm{zu} 300{ }^{\circ} \mathrm{C}$ erhitzt und mit komprimiertem Kohlendioxid oder Heptan ausgetragen. Am Vortag des Experiments wird die Anlage mehrfach mit Ethen gespült und mindestens 12 Stunden unter einem Ethendruck von 2500 bar belassen. Dies dient zum einen der Prüfung der Dichtigkeit, zum anderen können sich während dieser Zeitspanne Sauerstoffspuren und andere Verunreinigungen im überkritischen Ethen lösen und vor dem Experiment ausgetragen werden. Vor Beginn eines Experiments werden alle Sicherheitseinrichtungen geprüft und die Anlage optisch auf eventuelle Fehlfunktionen untersucht. Ein besonderes Augenmerk ist hierbei auf die Strömungskontrolle der Abluft zu richten, wo vor jedem Experiment die Alarmeinrichtung durch Schließen der Abluft zu kontrollieren ist.

\section{Durchführung eines Syntheseexperiments}

Unmittelbar vor dem Versuch wird der Druck in der Anlage abgelassen. Der Dosierzweig für Molmassenregler und Initiatoren wird mit den jeweiligen Substanzen gespült. Der Kühlwasserkreislauf der Kompressoren und des Rührantriebs wird geöffnet. Alle heizbaren Komponenten werden auf die jeweils gewählte Temperatur gebracht. Die dritte Kompressionsstufe wurde im Bypassbetrieb auf Betriebstemperatur gebracht. Ein zusätzlicher Heizkreislauf, der eine Temperierung der Kolbenmembranpumpe Lewa K3 am Pumpenkopf sicherstellt, wird auf ca. $40{ }^{\circ} \mathrm{C}$ geheizt, um eine Kristallisation der Methacrylsäure bzw. der Acrylsäure zu verhindern. Bei tieferen Temperaturen und im Druckbereich von bereits 300 bar kann es zu einem Ausfallen der Säure kommen, was zu einem Ausfall der Pumpe führen würde. Höhere Temperaturen könnten eine thermische Initiierung der Säure herbeiführen, was ebenfalls zum Ausfall des Dosierzweiges führen würde. ${ }^{[5]}$ Für eine detaillierte Beschreibung der Säuredosierung siehe Kapitel 3.1.5.

Zum Start des Copolymerisationsexperiments wird die entsprechende Säure in die dritte Kompressorstufe gefördert. Direkt daran anschließend wird der größtmögliche Ethenfluss gefördert, um möglichst schnell den Synthesedruck von bis zu 2500 bar zu erhalten. Bei geöffnetem Ventil des Massenflussreglers werden ca. $2 \mathrm{~kg} \cdot \mathrm{h}^{-1}$ Ethen gefördert. Das Feinventil wird soweit geschlossen, dass sich der Synthesedruck aufbauen kann. Der Rührmotor wird zu Beginn der Druckaufbauphase eingeschaltet. Der Massenflussregler wird auf den entsprechenden Sollwert geschaltet, in dieser Arbeit meist $1.8 \mathrm{~kg} \cdot \mathrm{h}^{-1}$, sobald ein Reaktionsdruck erreicht wird, der 500 bar unterhalb des gewünschten Synthesedrucks liegt. Aus Sicherheitsgründen wird bereits hier das Feinventil soweit geöffnet, dass sich ein 
signifikanter Druckverlust einstellt. Das dient der Überprüfung der Kapillaren auf Durchlässigkeit und gleichzeitig wird die Funktion des Feinventils sichergestellt. Der Reaktionsdruck wird manuell mit Hilfe des Feinventils reguliert. Die Initiatordosierung wird nun bei dem entsprechenden Synthesedruck ebenfalls in die dritte Kompressorstufe eindosiert. Nach Erreichen stationärer Betriebsbedingungen werden mindestens weitere 5 Verweilzeiten abgewartet (insgesamt ca. 15 Minuten) Anschließend wird innerhalb eines definierten Zeitraums eine Polymerprobe in der Abscheideeinheit aufgefangen.

\section{Beendigung eines Experiments}

Zur Beendigung der Polymersynthese wird zunächst die Initiatordosierung ausgestellt und gewartet bis die Reaktionstemperatur deutlich absinkt und sich im Bereich der Manteltemperatur des Reaktors stabilisiert. Darauf hin wird die Säureförderung gestoppt. Nach 5 Verweilzeiten werden die Kompressoren auf Bypass-Förderung geschaltet, und die Anlage wird über das Feinventil druckentlastet. Die Heizungen und Kompressoren werden abgeschaltet. Die Säure-Dosierpumpen und der Initiatorzweig werden mit einer Mischung aus Aceton und Heptan bei hohem Fluss gespült.

\section{Reinigung}

Das teilweise intensive Anhaften der untersuchten Ethen-(Meth)Acrylsäurecopolymere nach dem Erstarren auf Metalloberflächen, eine ansonsten anwendungstechnisch interessante Eigenschaft dieser Materialien ${ }^{[11]}$, erschwert die Reinigung der Hochdruckapparaturen. Die stärker (meth)acrylsäurehaltigen Probenreste lassen sich gut mit Aceton entfernen. Als günstige Reinigungsmethode bei Proben niedrigeren (Meth)Acrylsäuregehalts erwies es sich, die Polymerreste zunächst mindestens 30 Minuten in Isododekan bei möglichst hoher Temperatur aufquellen zu lassen. Danach können die Reste mit einer zylindrischen Messingdrahtbürste mechanisch entfernt werden. In früheren Experimenten ${ }^{[12]}$ konnte ein initiierender Einfluss des Isododekans technischer Reinheit nicht ausgeschlossen werden, deswegen wird die Anlage anschließend mit Aceton gespült und gegebenenfalls mit Pressluft nachgetrocknet. 


\subsubsection{Dosierung des Comonomers (Meth)Acrylsäure}

Die Dosierung von (Meth)Acrylsäure erfolgt im Regelfall bei einem Druck von bis zu 300 bar. Je nach Schwankungen des Reaktordrucks sind Dosierdrücke von bis zu 400 bar beobachtet worden. Ab einem Druck von 480 bar schaltet sich die Kolbenmembranpumpe automatisch auf Störung und stoppt die Förderung. Die Dosiereinheit der Säure ist mit einer optischen und akustischen Warneinrichtung ausgestattet. Das Ansprechen dieser Warnvorrichtung (Überschreiten des Maximaldrucks von 480 bar) ist über das computergesteuerte Menü der Kolbenmembranpumpe programmierbar. Für den Betrieb des Mini-Technikums zur Ethen-(Meth)Acrylsäure-Polymerisation ist diese Warnvorrichtung unerlässlich, da ein nicht bemerktes Pumpenversagen bei diesen Synthesebedingungen zu einer thermischen Zersetzung der Reaktanden führen kann (siehe Kapitel 3.1.7). Der zu erbringende Förderdruck der beiden Dosierpumpen ist abhängig davon welcher Reaktordruck eingestellt wird. Bei Polymerisationen im Bereich eines Reaktionsdrucks von 2500 bar steigt der Vordruck der dritten Kompressorstufe, also der Ausgangsdruck nach der zweiten Kompressionsstufe, teilweise auf über 400 bar an. Dieser Druck muss auch von den Dosierpumpen erreicht werden, was zu einigen Problemen und Ausfällen führen kann. Aufgrund dieser Tatsache wurden für weitere Experimente Drücke von maximal 2300 bar gewählt. Diese Entscheidung trug erheblich zur Stabilisierung der Messbedingungen bei, da Pumpenausfälle aufgrund eines zu hohen Vordrucks minimiert wurden.

Eine direkte Eindosierung in den Reaktor, wie es zum Beispiel mittels einer schrittmotorgesteuerten Spindelpresse möglich ist, ist bei Drücken von über 1000 bar, dem niedrigsten in dieser Arbeit verwendetem Druck, nicht möglich, da die Säuren bei Raumtemperatur und Drücken von 700 bar in fester Form vorliegen. ${ }^{[5]}$

Spezielle Herausforderungen an die Dosierpumpe werden in dieser Arbeit durch die zu fördernden Säuren gestellt. Mittels der im Arbeitskreis weit verbreiteten HPLC-Pumpen lassen sich diese Arten von Säuren nicht fördern. ${ }^{[5]}$ Auch bei der speziell für die Säuren beschafften Kolbenmembranpumpe ergeben sich zahlreiche Probleme. Der Dosierstrom reißt in vielen Fällen komplett ab. Die Pumpe zeigt einen Fehler „Störung Saugseite“ an. Das beruht auf einer Fehlfunktion der Kolbenrückstellung. Beim Ansaugvorgang strömt die Flüssigkeit nicht schnell genug in die Pumpe. Das hat zur Folge, dass die Pumpe die Förderung automatisch abbricht und somit wiederum kein kontinuierlicher Comonomerfluss mehr gewährleistet werden kann. Nach mehrmaliger Reinigung und 
Austausch der Ein- und Auslassventile der Kolbenmembranpumpe wurde keine Verbesserung erreicht. Nach Rückfrage bei der Herstellerfirma wurde eine Generalinspektion und ein Update der Software durchgeführt. Der Fehler „Störung Saugseite“ tritt nun zwar auch noch vereinzelt auf, aber ein großer Vorteil des SoftwareUpdates liegt darin, dass nun der Fehler „Störung Saugseite“ zwar angezeigt wird, die Pumpe aber weiterhin fördert. Der konstante Fluss konnte zweifelsfrei mittels einer gravimetrischen Überwachung belegt werden.

Methacrylsäure lässt sich in reiner Form über lange Zeiträume ohne größere Probleme fördern. Bei Komplettausfall der Pumpe führt ein Zerlegen des Pumpenkopfes und eine sehr sorgfältige Reinigung der Ein- und Auslassventile mit Aceton zur erneuten Einsatzbereitschaft. Dies kann mit etwas handwerklichem Geschick auch während eines laufenden Experiments durchgeführt werden. Es ist darauf zu achten, dass der Initiatorfluss gestoppt wird und der Säurezweig nach der Reinigung erneut gespült werden muss, da sich noch Rückstände des Acetons und Luft in den Kapillaren befinden können. Aufgrund des erheblichen Zeitverlusts und des Risikos, dass sich nach bereits langandauernden Experimenten der Reaktor zusetzen kann, ist diese Art der Reinigung nur zu Beginn eines Versuchstages empfehlenswert.

Acrylsäure stellt bekannter Weise erheblich größere Probleme dar. Das von Wittkowski ${ }^{[5]}$ vorgeschlagene Verfahren mittels eines Druckluftstroms das System auf $25{ }^{\circ} \mathrm{C}$ zu kühlen brachte hier keinen Erfolg. Auch der Einbau einer Kühlschleife, die aus einem spiralförmigen Plastikschlauch in einem mit Eiswasser gefüllten Erlenmeyerkolben besteht, bringt keine spürbare Besserung. Eine Erwärmung der Pumpe auf bis zu $70{ }^{\circ} \mathrm{C}$ führt zwar nicht zur Polymerisation der Acrylsäure, beeinflusst aber die Förderbarkeit ebenfalls nicht. Es erweißt sich hier als zweckmässig, eine Lösung von Acrylsäure in einem geeigneten Lösungsmittel anzusetzen. Da Cyclohexan als Lösungsmittel für den Initiator dient und möglichst wenige zusätzliche Komponenten dem System zugeführt werden sollen, wird hier eine Lösung bestehend aus jeweils gleichen Massenanteilen an Acrylsäure und Cyclohexan verwendet.

Als 50 \%ige Lösung ist eine Förderung über ein ausreichendes Zeitintervall möglich. Im Falle der Acrylsäure sollte das Experiment aufgrund von Zersetzungsreaktion nach spätestens 3 Stunden Säuredosierung beendet werden, da mehrmals nach exakt diesem Zeitraum eine Zersetzung aus noch ungeklärten Ursachen beobachtet wurde. 


\subsubsection{Dosierung der Initiatorlösung}

Üblicherweise wird bei der Homopolymerisation der Initiator mittels einer motorgesteuerten Spindelpresse direkt bei dem Synthesedruck, in den meisten Fällen bei 2000 bar $^{[7]}$ in den Reaktor gefördert. Bei Druckschwankungen führt das zu veränderlichen Initiatorzuflüssen. Gerade dieses System Ethen-(Meth)Acrylsäure neigt zur Zersetzung, sollte eine $\mathrm{zu}$ hohe Radikalkonzentration im Reaktor vorliegen. Deswegen wurde die Dosierung des Initiators ebenfalls wie die Säure bereits vor der dritten Kompressorstufe vorgenommen. Der Hauptgrund hierfür liegt in der geringen Schwankung des Vordrucks (Vordruck Stufe 3). Ein weiterer positiver Effekt ergibt sich nun durch die gleichen Druckbedingungen für die Säureförderung und für die Initiatorförderung. Erhöht sich der Vordruck, so müssen sowohl die Kolbenmembranpumpe (Säuredosierung) als auch die HPLC-Pumpe (Initiatordosierung) gegen diesen höheren Druck fördern. Die Überwachung des Initiatorflusses erfolgt gravimetrisch. Bei Verwendung der Spindelpresse wäre die Messgröße lediglich das verdrängte Volumen pro Zeiteinheit, wobei ein größerer Fehler in der Berechnung des Dosierstroms zu erwarten ist.

Die HPLC-Pumpe bietet weitere Vorteile gegenüber der Spindelpresse. Eine Dosierung ist auch bei höheren Flüssen über einen langen Zeitraum möglich und aufgrund der einfachen Nachfüllbarkeit der Initiatorlösung in einer Vorlage (Schraubdeckelglas) ist somit keine Unterbrechung der Dosierung notwendig, wie es im Falle der nur ca. $12 \mathrm{ml}$ fassenden Spindelpresse auftritt.

\subsubsection{Durchführung von Copolymerisationsexperimenten mit (Meth)Acryl- säure als Comonomer}

Nachdem in Kapitel 3.1.4 allgemein die Durchführung einer Hochdruckpolymerisation beschrieben ist, wird in diesem Abschnitt auf Besonderheiten der hier durchgeführten Copolymerisation eingegangen. Die Auswahl der Messbedingungen und Beobachtungen während des Experiments werden geschildert.

\subsubsection{Durchführung der Copolymerisation}

Ziel eines Versuchstages ist es, mindestens zwei Proben bei möglichst stark verschiedenen Drücken zu erhalten. Wie bereits in Kapitel 2 erwähnt, werden in dieser Arbeit 
Copolymere auf ihre statistische Anordnung von Monomerbausteinen untersucht. Proben, die in weiter Entfernung zur Phasengrenze, also weit im homogenen Bereich synthetisiert werden, zeigen eine rein statistische Anordnung der Monomerbausteine. Der Einbau erfolgt also zufällig unter Berücksichtigung der Copolymerisationsparameter. ${ }^{[13,14]}$ Für nahe an der Phasengrenze synthetisierte Proben wird eine nicht-statistische Anordnung der Monomerbausteine erwartet. ${ }^{[15,16]}$ Die (Meth)Acrylsäurebausteine werden häufiger als Dimerblock (oder höhere Oligomere) in das Copolymer eingebaut als die Statistik erwarten lässt.

\subsubsection{Hochdruckproben}

Im Normalfall wird jeweils mit einer „Hochdruckprobe“ begonnen, um Fouling zu vermeiden (siehe Kapitel 3.1.7.3). Hochdruckprobe bedeutet in diesem Zusammenhang, dass der Maximaldruck der Anlage ausgenutzt wird, um ein Polymer unter Bedingungen zu produzieren, die deutlich oberhalb der ermittelten Phasengrenze ${ }^{[10]}$ liegen. Wie bereits in Kapitel 3.1.5 beschrieben, werden für Hochdruckproben meistens Drücke von 2300 bar gewählt. Bei hohen Reaktionstemperaturen kann somit eine homogene Reaktionsführung sichergestellt werden. Auch bei eventuell einsetzenden Drucksprüngen besteht keine Gefahr, dass das System in einen heterogen Zustand verfällt. Die homogene Reaktionsführung wird auch mittels des eingelassenen Saphirfensters optisch überwacht. Zu Beginn der Reaktion ist der Reaktor innen klar und es lässt sich deutlich die Bewegung des Rührwerkes beobachten. Nach Zugabe von ausreichend Initiator kommt es zu einer deutlichen Temperaturerhöhung. Die Reaktionstemperatur, die direkt in der Reaktionsmischung gemessen wird, liegt ca. $30^{\circ} \mathrm{C}$ oberhalb der Manteltemperatur. $\mathrm{Zu}$ diesem Zeitpunkt weisen auch zwei andere Faktoren auf den Start der Reaktion hin. Zum Einem färbt sich der Reaktorinhalt nun schwarz und der Rührer ist nur noch schemenhaft zu erkennen. Dennoch lässt sich dieser Zustand optisch von einer heterogenen Reaktionsführung unterscheiden (siehe Niederdruck). Zum Anderen entstehen erhebliche Geräusche durch den Abscheidevorgang des entstehenden Copolymers, da bei Gesamtmonomerumsätzen von mehr als $10 \%$ synthetisiert wird. Der Umsatz ergibt sich automatisch aus dem zugegebenen Initiator und der Reaktionstemperatur. 


\subsubsection{Niederdruckproben}

Als Niederdruckproben werden Copolymere bezeichnet, die nahe der Phasengrenze synthetisiert werden. Der Druck wird ausreichend hoch gewählt, sodass gerade noch eine homogene Reaktionsmischung vorliegt. Der Reaktionsdruck soll aber auch möglichst tief liegen, um einen hohen Druckunterschied zwischen den Hoch- und Niederdruckproben zu erreichen. Für die meisten Copolymere stellt ein Druck von 1300 bar einen guten Kompromiss dar. Hierfür war es notwendig, die „Stufe“ der dritten Verdichtungseinheit zu verändern. Der Kompressor baut bis zu dieser Stufe kontinuierlich Druck auf. Danach wird mittels eines öldruckgesteuerten Ventils ein weiterer Druckaufbau verhindert. Erst wenn die zweite Kompressorstufe einen ausreichend hohen Druck aufgebaut hat (ca. 200 bar), öffnet dieses Ventil und ein Druckaufbau bis zu 3000 bar ist möglich. Während dieser Stufe ist es nicht möglich den Reaktordruck mittels Feinventil zu regulieren. Die Stufe lag anfänglich bei 1500 bar und somit nahe des für die in dieser Arbeit untersuchten Polymere interessanten Druckbereiches. Um keinerlei Störung durch diesen Effekt zu erhalten, wurde die Federvorspannung, mit der das Ventil in den Sitz gedrückt wird, geändert. Die Stufe wurde somit auf ca. 900 bar verlagert und eine problemlose Druckregulierung wurde realisiert.

Bei Reaktionstemperaturen von unter $240{ }^{\circ} \mathrm{C}$ ist es notwendig, aufgrund des Phasenverhaltens, einen höheren Synthesedruck zu wählen. Das kann visuell festgestellt werden. Die installierte Kamera liefert Bilder aus dem Inneren des Reaktors während der Polymerisation. Bei der Verwendung von Methacrylsäure färbt sich, wie oben bereits beschrieben, die Reaktionsmischung schwarz. Es ist deutlich zu erkennen, wenn eine heterogene Reaktionsführung vorliegt. Es kommt zum Ausfallen des entstehenden Copolymers, da es sich unter den gegebenen Reaktionsbedingungen nicht mehr in der Reaktionsmischung löst. Der Reaktor wird von kleinen weißen Wolken durchzogen. Aus diesem Phänomen lässt sich der englische Name „cloud point pressure“ ableiten. Des Weiteren kann ein minimaler Farbumschlag von schwarz zu schwarz-grau beobachtet werden. Bei ausreichender Druckerhöhung kommt es zur Auflösung der Wolken und die Farbe orientiert sich wieder in Richtung schwarz. Es ist darauf zu achten, dass der Zeitraum, in dem die heterogen Reaktionsführung vorliegt, minimiert wird, da es, wie beschrieben, zu einem Ausfall an Polymer kommt. Diese Polymere führen zum Reaktorfouling. Als Fouling bezeichnet man die Ablagerung von Polymer an den Reaktorwänden und am Rührkäfig. Diese Ablagerungen führen zur Verkleinerung des Reaktionsvolumens. Um nun einen konstanten Umsatz zu erhalten, bedarf es mehr 
Initiator. Das führt wiederum zu Veränderungen der Mikrostruktur der Polymere. Diese Beläge sind nur in sehr wenigen Fällen durch eine Druck- oder Temperaturerhöhung zu reduzieren und die vollständige Entfernung ist während eines Versuches nahezu unmöglich.

Bei sehr hohen Reaktionstemperaturen ist es möglich einen Druck von weniger als 1300 bar für die Niederdruckprobe zu wählen. Das Phasendiagramm ${ }^{[10]}$ dient hier als Anhaltspunkt für die zu erwartende Phasengrenze. Die Literaturdaten beruhen auf Messungen, in denen lediglich das Copolymer und Ethen vorlagen. Es wurden Mischungen hergestellt, die den ungünstigsten Fall widerspiegeln. Das bedeutet, der Gewichtsanteil an Polymer wurde so gewählt, dass die Werte für Temperatur und Druck, die zum Erreichen der Phasengrenze führen, maximal werden. In den hier durchgeführten Versuchen kommen Einflüsse durch das Comonomer und den Initiator zum Tragen. Der Gewichtsanteil des Polymers liegt in einem Bereich, in dem eine bessere Mischbarkeit der Komponenten vorliegt. Es ist also eine zusätzlich Absenkung der Druck- und Temperaturwerte für die Phasengrenze bei den hier durchgeführten Versuchen zu erwarten.

Mit diesem Vorgehen lässt sich der Temperaturbereich von 230 bis $300{ }^{\circ} \mathrm{C}$ abdecken. Bei tieferen Temperaturen zeigt das Phasendiagramm einen erheblichen Anstieg des für eine homogene Reaktionsführung benötigten Druckes. Im Bereich von 200 bis $210{ }^{\circ} \mathrm{C}$ sollte es theoretisch möglich sein, eine unstatistische Probe, also eine „Niederdruckprobe“ bei 2300 bar, herzustellen.

Bei geplanten Reaktionstemperaturen von $200{ }^{\circ} \mathrm{C}$ ist es notwendig eine Manteltemperatur von ca. $160^{\circ} \mathrm{C}$ einzustellen. Bei dieser Temperatur lässt sich die Reaktion mittels $\mathrm{TxF}$ praktisch nicht starten. Es wäre eine erheblich Menge an Initiator erforderlich, was vom Aufbau her auch realisierbar wäre, sobald aber die Reaktion startet, erhöht sich die Temperatur aufgrund der Reaktionswärme schlagartig und die Initiatoreffektivität steigt ebenfalls rasant an. Auch eine sofortige komplette Abschaltung der Initiatordosierung würde hier keine Verbesserung bringen, da die Konzentration an nicht zerfallenem Initiator im Reaktor sehr hoch ist. Man kann von aufgestautem nicht zerfallenem Initiator sprechen, der nun schlagartig zerfällt und Ketten startet. Das Risiko einer Zersetzung ist hier viel zu hoch und die Versuchsdurchführung so nicht realisierbar.

Eine Option wäre hier einen Initiator zu wählen, der eine optimale Temperatur im Bereich von $150{ }^{\circ} \mathrm{C}$ besitzt. Aufgrund der Vergleichbarkeit der Proben wurde davon abgesehen einen weiteren Initiator zu verwenden. 
Als praktikable Lösung erscheint ein Start der Reaktion bei einer hohen Temperatur. Die Manteltemperatur wurde zwischen 240 und $250{ }^{\circ} \mathrm{C}$ gewählt und ausreichend Initiator zugegeben um eine Reaktionstemperatur von ca. $260{ }^{\circ} \mathrm{C}$ zu erreichen. Nun wird sukzessive die Manteltemperatur verringert und der Initiatorfluss angepasst, so dass die Reaktion nicht erlischt. Reaktionstemperaturen von $210^{\circ} \mathrm{C}$ werden auch erreicht. Es handelt sich aber nicht um leicht $\mathrm{zu}$ kontrollierende Bedingungen. Sobald nicht ausreichend Initiator vorhanden ist, kommt es zu einem schnellen Abbruch der Reaktion, der sich in einem abrupten Temperaturabfall bemerkbar macht. Das verschiebt die Phasengrenze zu höheren Drücken. Um das Polymer weiterhin lösen zu können muss eine Druckerhöhung stattfinden, was allerdings hier nur sehr bedingt realisierbar ist, da bei höheren Drücken, die Dosierpumpen ausfallen (siehe Kapitel 3.1.5). Können die Druckänderungen die Reaktion nicht unmittelbar wieder starten, so kommt es unweigerlich zum Ausfall des Polymers und ein erneutes Überschreiten der Phasengrenze ist praktisch unmöglich.

Es gelang zwar, bei zwei Messungen jeweils Proben zu nehmen, aber die Temperatur variierte von 197 bis $256^{\circ} \mathrm{C}$ und Umsatzkonstanz war auch nicht gegeben. Aus diesem Grund wurden die Proben nur bedingt für weitere Auswertungen verwendet.

Erschwerend kam hinzu, das beide Messungen beendet werden mussten, da der Rührkäfig aufgrund des ausgefallenen Polymers sich aus seiner Verankerung riss und erheblichen Schaden anrichtete. Deswegen wurde von weiteren Versuchen bei niedrigen Temperaturen abgesehen.

\subsubsection{Initiatorlösung}

Bei den ersten Versuchsreihen wurde ein Initiatorcocktail verwendet, bestehend aus tertButylperoxyacetat $(\mathrm{TxF})$ und Di-tert-butylperoxid $(\mathrm{TxB})$. Diese beiden Initiatoren unterscheiden sich in ihrer optimalen Temperatur, $T_{\mathrm{opt}}$, TxF weist bei ca. $220^{\circ} \mathrm{C}$ die höchste Initiatoreffektivität auf, wo hingegen TxB erst bei $260^{\circ} \mathrm{C}$ die höchste Effektivität besitzt. Der Initiatorcocktail besteht also aus einer Komponente, die bereits bei der gewählten Manteltemperatur von $220{ }^{\circ} \mathrm{C}(\mathrm{TxF})$ die höchste Effektivität besitzt und somit zu Beginn der Reaktion für ausreichend Initiatorradikale sorgt. Der zweite Initiator (TxB) initiiert maximal bei einer Temperatur von $260{ }^{\circ} \mathrm{C}$, was bei den ersten Versuchsreihen (EMAA 1-12) der Reaktionstemperatur entspricht. Der Einsatz von lediglich einem Initiator kann Probleme mit sich bringen. Verwendet man ausschließlich TxB, so ist es erforderlich zu Beginn des Experiments relativ viel Initiator in das System zu geben. Nach 
dem Start der Reaktion erwärmt sich das Gemisch aufgrund der Reaktionsenthalpie. Das führt zu einer Zunahme der Initiatoreffektivität. Somit werden noch mehr Ketten gestartet, was wiederum zu einer höheren Temperatur führt. Die Reaktion beschleunigt sich selbst und es besteht das Risiko der Unkontrollierbarkeit. Dieser Verlust der Kontrolle endet in vielen Fällen mit der Zersetzung des Monomers Ethen zu Kohlenstoff. Diese Zersetzung belastet das Material des Technikums erheblich, da die Bauteile für eine kurze Zeit mit einem höherem Druck als beim normalen Betrieb belastet werden. Die Sicherheitsberstscheiben zerreißen bei einem Druck von 3009 bis 3289 bar (Herstellerangaben). Deswegen muss nach jeder Zersetzung die Länge der Reaktorbolzen kontrolliert werden. Sollten diese Bolzen sich um mehr als $1 \%$ ihres ursprünglichen Maßes gelängt haben, so sind sie durch neue Bolzen zu ersetzen. Als zeitaufwendigste Komponente einer Zersetzung ergibt sich die Reinigung. Alle Bauteile, die durch den entstandenen Kohlenstoff verunreinigt sind, müssen weitestgehend zerlegt und mittels eines geeigneten Reinigungsmittels, hier Aceton, gespült werden. Auch der Reaktor muss komplett zerlegt und gereinigt werden. Nach der Reinigung sind die Dichtflächen zu kontrollieren und das eingelassene Saphirfenster ist mittels Teflon zu dichten. Nach der Reinstallation der Hochdruckanlage ist diese unter erhöhter Vorsicht und mit großer Sorgfalt auf Dichtigkeit zu prüfen. Die Erfahrungen zeigen hier, das Polymere, die bei Experimenten erhalten werden, denen eine Zersetzungsreaktion vorausging, meist verunreinigt sind. Nach einem mehrstündigen Experiments und einer erneuten routinemäßigen Reinigung ist dieses Problem meistens behoben.

Bei einer gewählten Manteltemperatur von $240{ }^{\circ} \mathrm{C}$ und einem Comonomergehalt im Dosierstrom von $f_{\mathrm{MAA}}=0.006$ stellt sich nach Initiatorzugabe eine Reaktionstemperatur im Bereich von 260 bis $270{ }^{\circ} \mathrm{C}$ ein. Bemerkenswert ist hier, dass bei einer Rücknahme des Initiatorflusses die Temperatur erwartungsgemäß fällt, sich aber kein stabiler Betriebspunkt ergibt. Das bedeutet, die Reaktion ,geht aus“ und lässt sich nicht mehr mit ausreichenden Umsätzen durchführen. Die Umsätze fallen signifikant auf einen Bereich deutlich unter $10 \%$ ab und die Reaktionstemperatur stabilisiert sich in Temperaturbereichen, die teilweise unterhalb der Manteltemperatur liegen. Bei Zugabe größerer Initiatormengen zeigt sich ein ähnlicher Effekt nur diesmal mit einem Temperaturanstieg. Das bedeutet, die Reaktionstemperatur steigt schnell an und strebt zielgerichtet gegen den apparativen Grenzwert von $300{ }^{\circ} \mathrm{C}$. Hier droht eine oben beschriebene Zersetzung. Aus diesem Grund ist es sinnvoll, den Initiatorfluss zu reduzieren, so dass man eine stabile 
Reaktionsführung im Bereich von 260 bis $270{ }^{\circ} \mathrm{C}$ erhält. Der Umsatz stellt sich somit automatisch ein und kann nur minimal durch die Regulierung des Initiatorflusses kontrolliert werden. Andernfalls lässt sich kein stabiler Betriebspunkt finden und das bedeutet, dass sich im Reaktor keine stationären Bedingungen ergeben. Diese stationären Bedingungen sind für den gleichmäßigen Probenaufbau unerlässlich und werden mittels Umsatzkonstanz über zwei bis drei Proben ermittelt. Einen Hinweis auf stationäre Reaktionsführung bietet auch die Reaktionstemperatur. Eine gewisse Fluktuation der Temperatur lässt sich unter diesen, im Vergleich zur Homopolymerisation oder Copolymerisation bei differentiellem Umsatz, anspruchsvollen Polymerisationsbedingungen, wie zum Beispiel hoher Umsatz und hoher Comonomerfluss, nicht vermeiden. Diese Schwankungen können im Bereich von bis $\mathrm{zu} \pm 8^{\circ} \mathrm{C}$ liegen. Sollte sich aber eine Tendenz in den Temperaturschwankungen abzeichnen, ein permanenter Temperaturanstieg oder -abfall, so ist davon auszugehen, dass sich keine stationären Bedingungen eingestellt haben.

\subsubsection{Verwendung eines Initiatorcocktails}

Die Verwendung eines Initiatorcocktail kann unter Umständen Temperaturschwankungen und den Verlust des stabilen Betriebspunktes noch begünstigen, da bereits geringe Temperaturänderungen zu einer Aktivierung eines verwendeten Initiators führen können. Aus diesem Grund wurde nach 6 durchgeführten Experimenten auf die Verwendung eines Cocktails verzichtet. Stattdessen wurde meistens TxF als Initiator gewählt. Somit führen Temperaturerhöhungen, induziert durch zum Beipiel Druckschwankungen, nicht mehr zu einer Erhöhung der Initiatoreffektivität.

\subsubsection{Initiatorverbrauch bei Hoch- und Niederdruckproben}

Es zeigt sich während der Polymerisation, dass eine Druckabhängigkeit des Initiatorverbrauchs vorliegt. Bei Druckreduktion um 1000 bar, kann sich der Initiatorverbrauch verdoppeln. Genaueres befindet sich im Kapitel 4.2 und 7.3. Bei einigen Polymerisationen ist es sinnvoll einer Temperaturänderung entgegen zu wirken, indem eine leichte Variation des Reaktionsdruckes vorgenommen wird. Hierbei wird davon 
ausgegangen, dass sich Druckschwankungen von \pm 50 bar nicht im signifikanten Maß auf das Produkt auswirken.

\subsubsection{Probenherstellung unter adiabatischen Bedingungen}

Es wurden Copolymerproben unter adiabatischen Bedingungen hergestellt. Der Vorteil dieser Reaktionsführung liegt in der Minimierung der Foulingprozesse. Des Weiteren ist der Umsatz hier nur von der Temperatur abhängig. Als Temperaturen wurden 240 und $260{ }^{\circ} \mathrm{C}$ gewählt. Für beide Fälle wurde TxF als Initiator gewählt. Bei einer Temperatur von $240{ }^{\circ} \mathrm{C}$ zeigte sich keine Druckabhängigkeit des Umsatzes. Für die höhere Temperatur ergaben sich bei Druckvariation Differenzen im Umsatz. Das könnte daran liegen, dass hier in einem Temperaturbereich gearbeitet wird, der deutlich über der optimalen Temperatur von TxF liegt und eventuell keine optimale Durchmischung der Reaktionslösung mehr vorliegt. TxB sollte für diese Bedingungen ein besser geeigneter Initiator sein. Die durchgeführten Experimente mit TxB als Initiator blieben aber erfolglos.

\subsubsection{Hergestellte Polymerproben}

Die in dieser Arbeit synthetisierten Proben lassen sich in vier Bereiche gliedern. Begonnen wurde mit einer Variation des Methacrylsäuregehalts bei einer möglichst gleichbleibenden Reaktionstemperatur von $260{ }^{\circ} \mathrm{C}$. Hier konnten drei verwertbare Probenreihen, bestehend aus mindestens jeweils einer Hoch- und Niederdruckprobe, erhalten werden. Auf diese Resultate aufbauend wurden zwei Probenreihen unter adiabatischen Bedingungen hergestellt. Zum Abschluss des Methacrylsäurekapitels wurde eine Temperaturvariation von 200 bis $300{ }^{\circ} \mathrm{C}$ durchgeführt.

Als Vergleichssystem für die Methacrylsäure dienen die im letzten Hauptbereich hergestellten Ethen-Acrylsäure Copolymere. Die Analyse dieser Acrylsäureproben ist der Inhalt einer parallel angefertigten Arbeit. 


\subsection{Experimentelle Methoden zur Ermittlung mikro- struktureller Eigenschaften}

\subsubsection{Kalorimetrische Untersuchungen}

Die DSC-Analyse („Differential Scanning Calometry“) gehört wie die TGA (Thermogravimetrie) und DMA (Dynamisch-mechanische Analyse) zu den thermischen Analysemethoden. Hierbei werden physikalische und chemische Eigenschaften einer Substanz als Funktion der Temperatur oder Zeit gemessen.

Die DSC, auch als Leistungs-Differenz-Kalorimetrie bezeichnete thermische Analysenmethode, wurde erstmals 1964 von Watson und O`Neil beschrieben. ${ }^{[17]}$ Das Kalorimeter besteht im Wesentlichen aus einer Ofenzelle, in welcher parallel eine Probe und eine Referenz vermessen werden (siehe Abbildung 3.3).

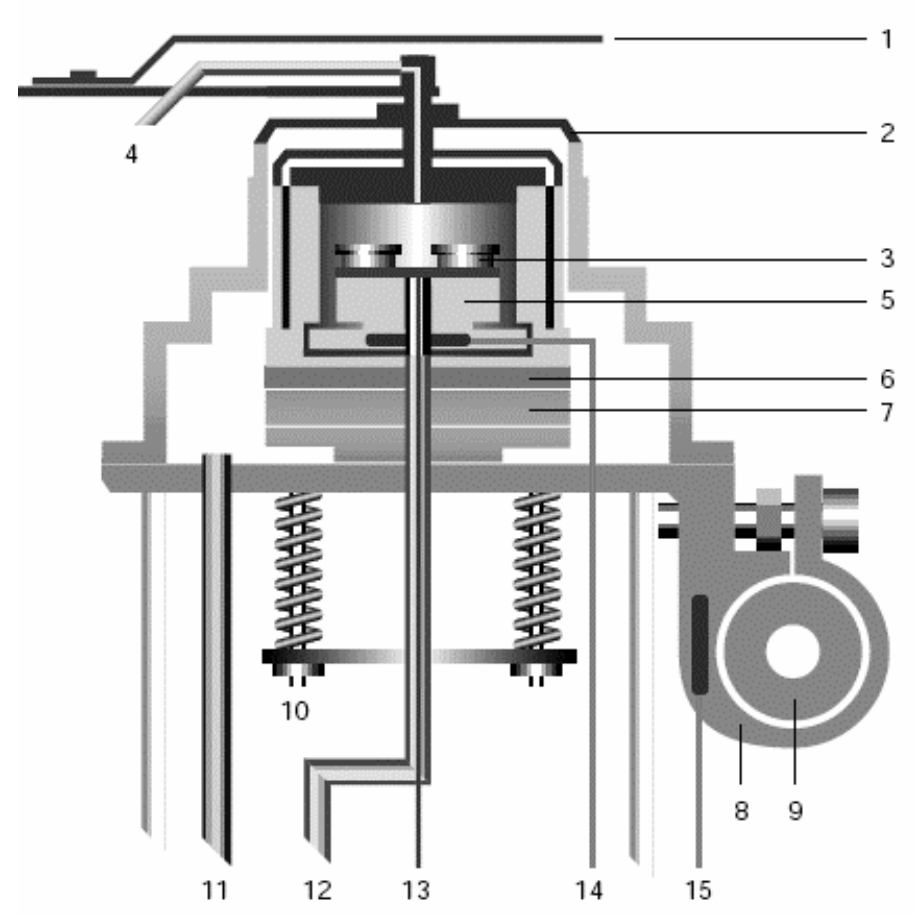

1 Hitzeschutzschild

2 autom. Ofendeckel.

3 Tiegel auf DSC-Sensor (links:

Probe, rechts: Referenz)

4 Spülgasanschluss

5 Silberofen

6 Flachheizung

7 Wärmewiderstand

8 Kühlflansch

9 Kühlfinger

10 Druckfeder

11 Trockengaseinlass

12 Spülgaseinlass

13 DSC-Signal

14 Pt100 Ofen

15 Pt100 Kühlung

Abbildung 3.3: Schematische Darstellung der elementaren Bestandteile einer DSCApparatur. ${ }^{[7]}$ 
Beide werden durch jeweils eine getrennte elektrische Widerstandsheizung zeitgleich aufgeheizt bzw. abgekühlt. Die gesuchte Messgröße, der differentielle Wärmefluss als Funktion der Zeit, wird aus der Differenzleistung der Widerstandsheizungen (Probenheizung und Referenzheizung) ermittelt. Die Messergebnisse werden in Form eines Thermogramms, welches für die untersuchten Substanzen spezifisch ist, protokolliert. Das Thermogramm gibt Auskunft über die Änderung der spezifischen Wärmekapazitäten der vermessenen Materialien.

Im Gegensatz zu Metallen besitzen teilkristalline Polymere keine scharfen thermodynamischen Phasenübergänge 1. Ordnung (Schmelzen, endotherm; Kristallisation, exotherm), sondern relativ breite Übergangsbereiche. Ursache dafür ist, dass teilkristalline Polymere aus Kristalliten mit unterschiedlichen Lamellendicken aufgebaut sind. Zu Beginn des Schmelzbereichs, etwa bei der Anfangstemperatur des Schmelzpeaks, schmelzen zuerst die weniger perfekten, also die dünneren Kristallite auf. Die Lage des Peakmaximums stellt dann die Temperatur dar, bei der die meisten Kristallite aufschmelzen. An der Endtemperatur des Schmelzpeaks sind schließlich alle Kristallite aufgeschmolzen, unabhängig von ihren Lamellendicken. Am Schmelzpeak eines teilkristallinen Polymers lässt sich also keine Schmelztemperatur im eigentlichen Sinne ermitteln. Zur Charakterisierung werden stattdessen Punkte wie beispielsweise die Anfangstemperatur des Schmelzpeaks, die Temperatur des Peakmaximums oder die Endtemperatur des Schmelzpeaks ermittelt. In der Praxis bedient man sich häufig der Temperatur des Peakmaximums als Schmelztemperatur, da sie meist charakteristisch für das untersuchte Polymer ist. Auch die Temperatur des Glasübergangs lässt sich nicht trivial aus dem Peakmaximum ermitteln. In dieser Arbeit werden Tangenten (Abbildung 3.4) an der Kurvenverlauf angelegt und aus dem Schnittpunkt dieser Tangenten wird die Glasübergangstemperatur bestimmt. 


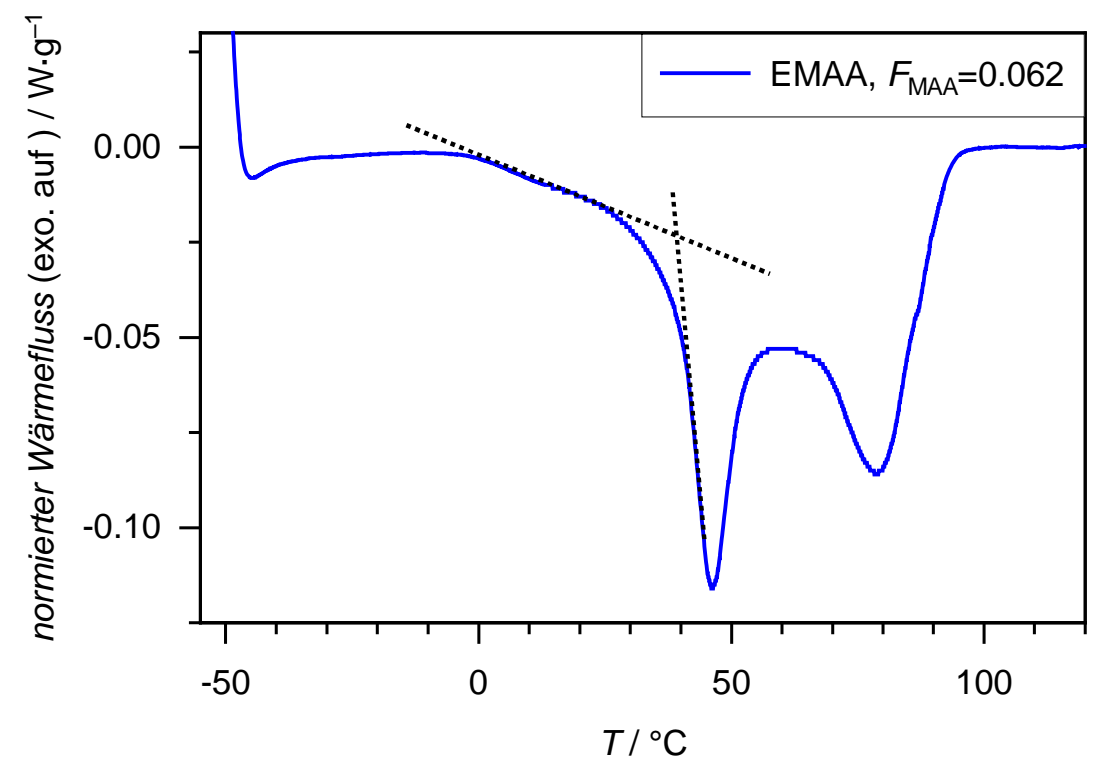

Abbildung 3.4: Typisches Thermogramm für die in dieser Arbeit synthetisierten Copolymere (EMAA 3.4, 2300 bar). Für den Glasübergang lässt sich mit Hilfe der perforierten Linien eine Temperatur bestimmen.

Alle in dieser Arbeit vorgestellten DSC-Daten wurden auf einem Gerät (DSC 820) des Herstellers Mettler-Toledo mit angeschlossenem Kryomat RUK 90 (Lauda) bestimmt (kalibriert gegen einen Indiumstandard bei einem Temperaturgradienten von $5{ }^{\circ} \mathrm{C} \cdot \mathrm{min}^{-1}$ : $156.6{ }^{\circ} \mathrm{C}, 28.71 \mathrm{~J} \cdot \mathrm{g}^{-1}$ ). Die Messzelle wurde mit gasförmigem Stickstoff der Reinheit 4.6 (Linde) bei einem Fluss von $14 \mathrm{ml} \cdot \mathrm{min}^{-1}$ gespült. Es wurden jeweils Probenmengen zwischen 10 bis $30 \mathrm{mg}$, typischerweise $15 \mathrm{mg}$, in $40 \mu \mathrm{l}$ Aluminiumtiegeln (Mettler-Toledo) vermessen.

Es wurde eine Vielzahl von Messprogrammen entworfen. Das geeignetste Programm sieht eine schnelle Abkühlung auf $-45^{\circ} \mathrm{C}$ (Starttemperatur) vor. Anschließend wird die Polymerprobe für $180 \mathrm{~s}$ bei der Starttemperatur gehalten. Nun wird bis zum Schmelzpunkt, der in den meisten Fällen im Bereich von $120{ }^{\circ} \mathrm{C}$ liegt, mit einer Heizrate von $5{ }^{\circ} \mathrm{C} \cdot \mathrm{min}^{-1}$ der Wärmefluss als Funktion der Temperatur gemessen. Auf ein vorheriges Aufschmelzen der Polymerproben wird in den meisten Fällen verzichtet. Die Angleichung der thermischen Vorgeschichte vor der eigentlichen Messung ist besonders in Fällen sinnvoll, wenn die Proben aus unterschiedlichen Herstellungsverfahren stammen. Beispielsweise kristallisiert ein Polymer beim Abscheidevorgang während der Produktion anders, als wenn es aus der Schmelze unter Druck extrudiert wird oder aus Lösung auskristallisiert. Aufgrund der weitestgehend gleichen Vorgeschichte der Polymerproben ist es in dieser Arbeit nicht notwendig eine Ausgleichung vorzunehmen. Das ist anhand von durchgeführten Messungen, wo die Heiz- und Kühlzyklen mehrmals durchlaufen wurden, 
nachgewiesen (Kapitel 4.10.4). Um eine direkte Vergleichbarkeit der Proben zu erreichen, wurden Normierungen an den erhaltenen Thermogrammen durchgeführt, die in Kapitel 4.10 beschrieben sind.

\subsubsection{FT-IR-spektroskopische Untersuchungen}

Als eine wichtige Technik der Polymeranalytik ist die FT-IR-Spektroskopie geeignet, um Informationen über Verzweigungen, Vernetzungen, Kristallinität und Restmonomergehalt zu erhalten. ${ }^{[18,19,20]}$ Für Copolymere ist es zudem möglich, die Zusammensetzung IR-spektroskopisch zu ermitteln.

In dieser Arbeit wurden von den untersuchten Copolymersystemen IR/NIR-Spektren aufgenommen, um die Copolymerzusammensetzungen zu ermitteln.

Zur Ermittlung der Zusammensetzung wurde in dieser Arbeit analog der Methode von Buback et al. ${ }^{[21]}$ das Verhältnis charakteristischer Absorbanzen der Comonomergruppen im IR-Spektrum verwendet. Die Spektren wurden anfänglich an zu Folien verarbeiteten Polymerproben aufgenommen. Diese Polymerfolien wurden mit einer beheizten, hydraulischen Presse (Specac Ltd.) hergestellt. Hierzu werden 20 bis 100 mg des Polymers zwischen zwei Teflonfolien von $0.1 \mathrm{~mm}$ Stärke in ein Presswerkzeug gelegt und zwischen die Druckplatten der Presse gebracht. Das Polymer wird darin auf $150{ }^{\circ} \mathrm{C}$ erhitzt und mit einer Kraft von $3 \cdot 10^{6} \mathrm{~N}$ gepresst. Nach etwa 2 Minuten wird das Presswerkzeug entnommen und in einem wassergekühlten Einsatz auf Zimmertemperatur temperiert. Die erhaltene Polymerfolie wird vorsichtig von den Teflonfolien getrennt und auf einem Kartonträger fixiert. Im Fall von Proben mit hohem Gehalt an Methacrylsäure tritt z. T. das Problem auf, dass sich die Teflonfolien aufgrund des zähflüssig klebrigen Charakters dieser Copolymere nicht abziehen lassen, ohne die gepressten Folien gleich wieder zu zerstören. Zur Umgehung dieser Problematik haben sich folgende Vorgehensweisen bewährt:

a) In einigen Fällen gelingt es, die Proben mitsamt den beiden Teflonfolien durch Eintauchen in flüssigen Stickstoff kurzzeitig so weit $\mathrm{zu}$ verhärten, dass sie vom Teflonmaterial getrennt werden können.

b) Spektren von sehr weichen Proben können an Probenmaterial aufgenommen werden, welches durch Zusammenpressen zwischen zwei $\mathrm{CaF}_{2}$-Fenstern in Filmform vorliegt.

c) Die Proben lassen sich mit den anhaftenden Teflonfolien vermessen. Hierbei muss das Ergebnisspektrum noch um die Absorbanz der Teflonfolien korrigiert werden. Da die 
Teflonfolien zum Teil durch das Pressen ebenfalls dünner werden, kann es erforderlich sein, eine Gewichtung der Teflonspektren vorzunehmen.

Die vorbereiteten Polymerfilme werden auf einem Halter in den Probenraum des Spektrometers gebracht. Die IR-Spektren werden mit einem Fourier-TransformSpektrometer (Bruker IFS 88), dessen Innenraum kontinuierlich mit wasser- und kohlendioxidarmer Pressluft gespült wird, aufgenommen. Es wird folgender optischer Aufbau verwendet: Globar-Lichtquelle, Silicium-beschichteter Calciumfluorid-Strahlteiler und DTGS-Detektor. Die Spektrenaufnahme und -verarbeitung wird über einen PC mit dem Programm OPUS (Spektrometer-Software, Bruker) gesteuert. Die im Wellenzahlenbereich von 1300 bis $8000 \mathrm{~cm}^{-1}$ ermittelten Spektren werden durch Coaddition von 100 Interferogrammen, mit anschließender Fourier-Transformation (Blackman-Harris 3Term Apodisierungsfunktion) zur Bestimmung des Einkanalspektrums und Berechnung des Absorbanzspektrums mit dem Leerkanalspektrum erhalten.

Aufgrund der erwähnten Probleme der Probenpräparation wurde nach Alternativen gesucht. Zu einem späteren Zeitpunkt dieser Arbeit stand eine weitere Messmethode, die ATR-FT-IR-Spektroskopie zur Verfügung. ATR steht für abgeschwächte Totalreflexion (Attenuated Total Reflection). Da hier nicht in Transmission sondern in Reflexion gemessen wird, ist die Dicke der zu untersuchenden Probe nicht mehr relevant. Als Träger für die Substanzen dient ein Diamantkristall, der fest in der ATR-Einheit (MVP 2 StarTM, $^{\mathrm{TM}}$ Harrick) verbaut ist. ${ }^{[2]}$ Die Probe wird mittels einer drehmomentüberwachten Schraubvorrichtung arretiert. Da es sich bei den Polymerproben um Festkörper handelt, bedarf es hier keinerlei Probenpräparation.

Buback et al. haben eine Methode vorgestellt, die das Verhältnis zweier Absorbanzen mit unabhängig bestimmten Copolymerzusammensetzungen korreliert. ${ }^{[21]}$

Hierbei werden die $\mathrm{C}-\mathrm{H}$ Absorbanz und die $\mathrm{C}=\mathrm{O}$ Absorbanz der Polymere im Bereich zwischen 1800 und $1400 \mathrm{~cm}^{-1}$ verwendet. Bei $1700 \mathrm{~cm}^{-1}$ ist der $\mathrm{C}=\mathrm{O}$ Grundton der Säuren zu erkennen. Die Absorbanz um $1480 \mathrm{~cm}^{-1}$ ist auf eine C-H Schwingung, und zu einem geringerem Anteil auf die Absorbanz von O-H Gruppen (Maximum bei ca. $1410 \mathrm{~cm}^{-1}$ ) zurückzuführen.

Für die Korrelation von Absorbanzdaten und der aus der Elementaranalyse ermittelten Copolymerzusammensetzung eignet sich folgende Gleichung. ${ }^{[23]}$

$$
\frac{\int(\mathrm{CO})}{\int(\mathrm{CH}+\mathrm{OH})}=\frac{F_{\mathrm{MAA}}}{a+F_{\mathrm{MAA}} \cdot b}+c \quad \text { Gleichung } 3.1
$$




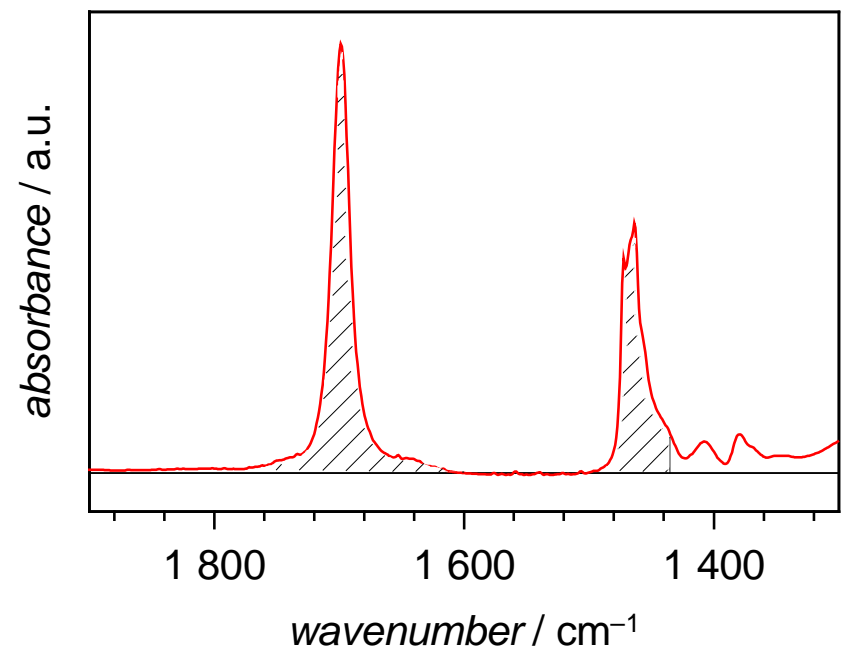

Abbildung 3.5: IR/NIR-Absorbanzspektrum von Poly(Ethen-co-Methacrylsäure).

In Tabelle 3.2 sind die verwendeten Integrationsmethoden angegeben. Die Integration erfolgt ebenfalls mittels des Programms OPUS mit der Methode E, das bedeutet, die Integrationsgrenzen und die vier Grundlinienpunkte für die Basislinie werden festgelegt.

\begin{tabular}{ccc}
\hline Copolymer & \multicolumn{2}{c}{ Integrationsgrenzen } \\
& $\mathrm{C}=\mathrm{O}$ Bande $/ \mathrm{cm}^{-1}$ & $\mathrm{CH}, \mathrm{OH}$ Bande $/ \mathrm{cm}^{-1}$ \\
\hline EMAA & 1950,1550 & 1530,1435 \\
EAA & 1850,1550 & 1530,1435 \\
\hline
\end{tabular}

\begin{tabular}{ccccc}
\hline Copolymer & \multicolumn{4}{c}{ Grundlinienpunkte } \\
& $\mathrm{C}=\mathrm{O}$ Bande $/ \mathrm{cm}^{-1}$ & $\mathrm{CH}, \mathrm{OH}$ Bande $/ \mathrm{cm}^{-1}$ \\
\hline EMAA & $1970,1950,1550,1520$ & $1550,1545,1535,1530$ \\
EAA & $1850,1820,1550,1520$ & $1550,1545,1535,1530$ \\
\hline Copolymer & \multicolumn{4}{c}{ Koeffizienten } \\
& $\mathrm{a}$ & $\mathrm{b}$ & $\mathrm{c}$ & $F_{(\mathrm{M}) \mathrm{AA}}$ Bereich \\
\hline EMAA & 0.0272 & 0.0474 & 0.4117 & $0-0.15$ \\
EAA & 0.0089 & 0.1665 & -0.574 & $0.025-0.08$ \\
\hline
\end{tabular}

Tabelle 3.2: Integrationsmethoden und Koeffizienten nach Gleichung 3.1 zur Korrelation von IR-Spektren und Copolymerzusammensetzung, $F_{(M) A A}$, für Poly(Ethen-coMethacrylsäure) (EMAA) und Poly(Ethen-co-Acrylsäure) (EAA). ${ }^{[5]}$ 


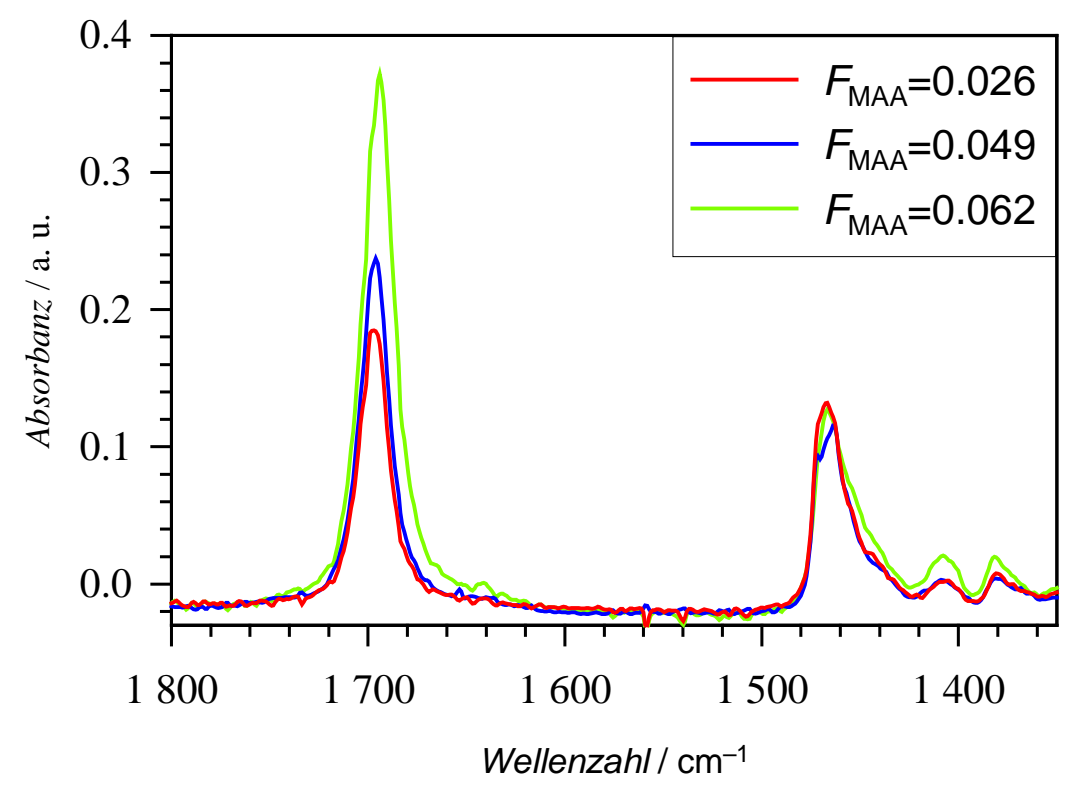

Abbildung 3.6: ATR-FT-IR Spektren für Copolymere mit variierendem Methacrylsäuregehalt.

\subsubsection{Dynamisch-mechanische Analyse}

\subsubsection{Aufbau der DMA}

Die dynamisch-mechanische Analyse (DMA) dient zur Bestimmung der viskoelastischen Eigenschaften einer makroskopischen Probe. Die Untersuchungen an Polymerproben wurden im Rahmen des Graduiertenkollegs „Spektroskopie und Dynamik molekularer Knäuel und Aggregate“ in Zusammenarbeit mit der Arbeitsgruppe von Herrn Prof. Samwer, I. Physikalisches Institut, in Göttingen durchgeführt.

Die verwendete DMA 7 der Firma Perkin-Elmer bietet eine Vielzahl von Geometrien für Proben im festen Zustand. Das Funktionsprinzip beruht stets darauf, dass eine Probe einer statischen, oszillierenden oder sich zeitlich ändernden mechanischen Spannung ausgesetzt und der Verlauf ihrer Deformation beobachtet wird. Im Folgenden soll die Handhabung der DMA beschrieben werden, mit besonderem Augenmerk auf Methoden, bei denen die Anweisungen im Handbuch ${ }^{[24]}$ verändert oder erweitert worden sind, sowie die benutzten Messmodi uniaxiale Dehnung und Kompression. Den Umgang mit der Windows ${ }^{\circledR}$ basierten Software beschreibt die in das Messprogramm integrierte Hilfsfunktion. 


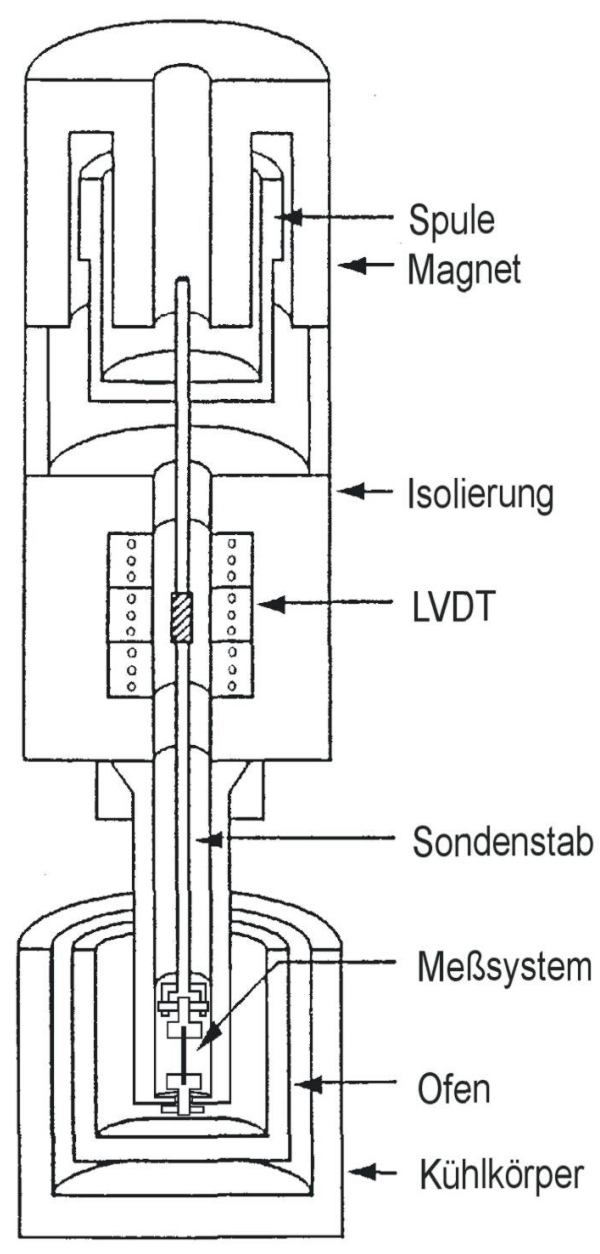

Abbildung 3.7: Schematischer Aufbau der DMA ${ }^{[25]}$

Der Aufbau der DMA 7 ist in Abbildung 3.7 schematisch dargestellt. Die Probe ist in einem variabel wählbaren Meßsystem fixiert und über den Sondenstab mit dem Linearmotor (Spule und Magnet) verbunden. Dieser kann dynamisch mit 0.01 bis $51 \mathrm{~Hz}$ oder mit konstanter Kraft von bis zu $1 \mathrm{~N}$ an der Probe ziehen oder diese komprimieren. Beste Ergebnisse lassen sich erzielen im Frequenzbereich zwischen 1 bis $20 \mathrm{~Hz}$ bei Kräften oberhalb $100 \mathrm{mN}$. Die resultierende Amplitude und Dehnung wird mittels eines linear variablen Differential-Transformators (LVDT) detektiert. Dieser arbeitet bei einer konstanten Temperatur von $45^{\circ} \mathrm{C}$, um ein Driften des Positionssignals zu minimieren und muss daher zunächst 45 Minuten warmlaufen und gut isoliert werden. Die Probe selbst kann definiert gekühlt oder geheizt werden, wobei ein Argon-Strom im Sondenrohr vom Ofen entkoppelt. Die den LVDT maximal erreichbare Temperatur liegt bei $500{ }^{\circ} \mathrm{C}$ im Ofen mit $28 \mathrm{~mm}$ Durchmesser und $1000{ }^{\circ} \mathrm{C}$ im Ofen mit

$15 \mathrm{~mm}$ Durchmesser. Die Kühlung erfolgt durch flüssigen Stickstoff, wodurch Probentemperaturen von bis $\mathrm{zu}-150{ }^{\circ} \mathrm{C}$ erzielt werden. Bei der Stickstoffkühlung erfolgt die Durchflussreglung automatisch durch ein Steuergerät mit Temperaturfühler vom Typ CCA 7. Zur Vermeidung von Kondenswasser ist es unabdingbar die DMA mit Stickstoffkühlung in einer mit Stickstoff oder Argon gefüllten Handschuhbox zu betreiben. Dies dient zusätzlich zum Argon-Strom im Sondenrohr zur Vermeidung von Oxidationseffekten. Die Kontrolle des Wasser- und Sauerstoffgehalts sowie die Abscheidung dieser Substanzen erfolgt durch eine Anlage vom Typ MB 20G der Firma MBraun. Da in einer wasserarmen Schutzgasatmosphäre (Wassergehalt $<5 \mathrm{ppm}$ ) Silikonschläuche auch ohne mechanische Beanspruchung brechen, wird die StickstoffZuleitung durch nicht kunststoffgedichtete Heißwasserschläuche aus Metallgewebe realisiert, die ökonomische Vorteile gegenüber der Faltenschlauch-Variante, bei akzeptabler Gasdichtigkeit, bieten. 
Die Ansteuerung der DMA und das Auslesen der Messgrößen erfolgt durch ein Steuergerät vom Typ TAC 7/DX, das seinerseits mit einem Computer verbunden ist. Die verwendete Version 4 der Pyris Software von Perkin Elmer benötigt dabei zwingend das Betriebssystem Windows 2000 mit Servicepaket 2. Auf diese Weise werden alle programmierbaren Parameter (Kraft, Frequenz, Heizrate) und die detektierten Größen (Änderung und Amplitude des Positionssignals des LVDT, Temperatur) aufgezeichnet. Anregung und Positionsänderung sind bei dynamischen Messungen um den Winkel $\delta$ phasenverschoben. Der Phasenwinkel ist damit direkt messbar, alle anderen Größen, die zur Charakterisierung der viskoelastischen Eigenschaften der Probe berechnet werden, benötigen eine genaue Kenntnis der Probengeometrie und deren Abmessungen.

\subsubsection{Kalibrierung}

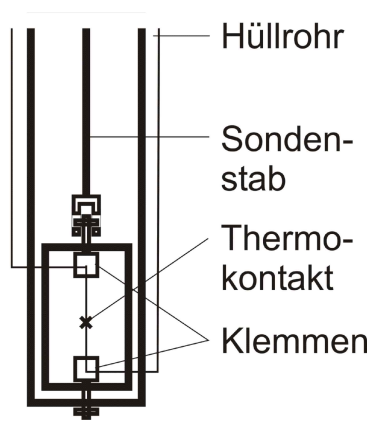

Abbildung 3.8:

Kalibriervorgang ${ }^{[25]}$
Die Kalibrierung der DMA erfolgt in sechs Schritten. Bei der „DMA Calibration“ werden neben grundsätzlichen Funktionstests auch die Reibung und die Trägheit der DMA bestimmt. Dazu wird ein 3-Punkt-Biegegestänge benutzt. Beim Verwenden von Quarzgestängen für die eigentlichen Messungen sollten auch während der Kalibrierung Quarzgestänge benutzt werden. Des Weiteren ist die Kalibrierung temperaturabhängig und sollte bei der Basistemperatur durchgeführt werden. Zur Maximierung der Empfindlichkeit des System wird die Reibung minimiert. Eine genaue Beschreibung des Verfahrens findet sich bei Hachenberg. ${ }^{[25]}$ Die Kalibrierung der Temperatur beim Arbeiten mit Stickstoff wird durch eine Kalibrierung mit einem Thermoelement am Probenort korrigiert. Den Aufbau zeigt Abbildung 3.8. Es ist zu beachten, dass für jede benutzte Heizrate eine Kalibrierung der Temperatur durchgeführt werden muss. Die Ofenkalibrierung zur Verringerung des Unterschieds zwischen Soll- und Ist-Temperatur wird gemäß Handbuch durchgeführt.

\subsubsection{Die Messmodi uniaxiale Kompression und Dehnung}

Je nach zu messendem Material bieten sich unterschiedliche Messmodi der DMA an. Eine Einführung, welcher Modus zu welcher Probe passt, gibt ${ }^{[26]}$. 
Für ein Polymer bietet sich die Kompression zwischen zwei parallelen Platten an, solange es nicht zu hart ist. Die uniaxiale Dehnung wäre für dünne, homogene Filme anwendbar. Weit unterhalb der Glastemperatur wäre auch eine Biegemethode erfolgversprechend, dies stellt aber hohe Ansprüche an die Qualität eines Films oder Biegebalkens, und der Einbau sollte bei Temperaturen unter der Glastemperatur stattfinden. Aus diesem Grund und wegen der Einsetzbarkeit oberhalb der Glastemperatur, wird für EMAA hauptsächlich die Kompression zwischen zwei parallelen Platten verwendet (Abbildung 3.9b). Nach erfolgreichen Kompressionmessungen werden auch Zugversuche durchgeführt. Die Probenpräparation für die verschiedenen Messmodi findet sich in Kapitel 3.2.3.4.

a)

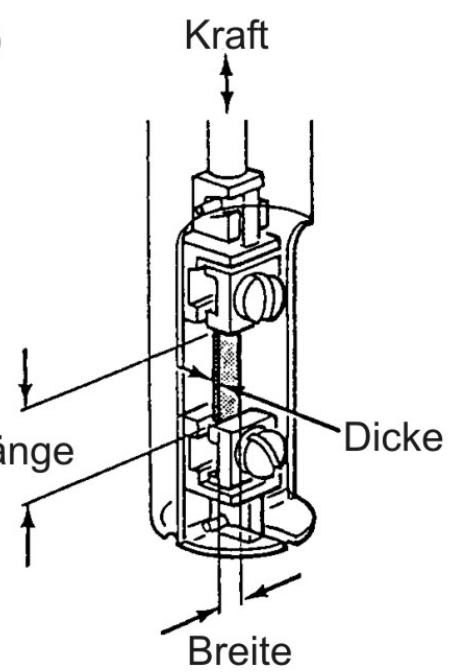

c)

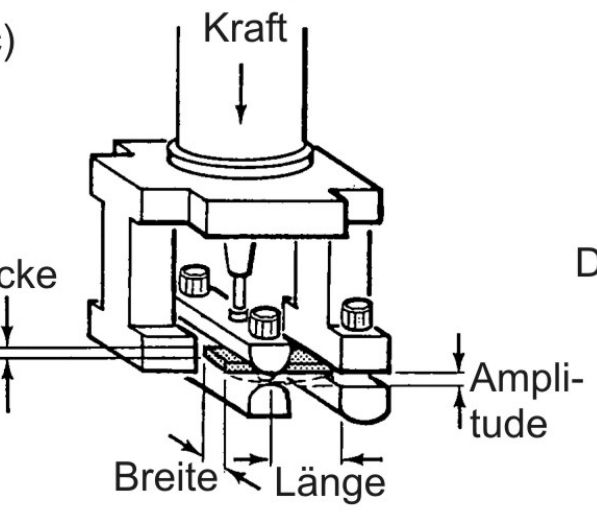

b)

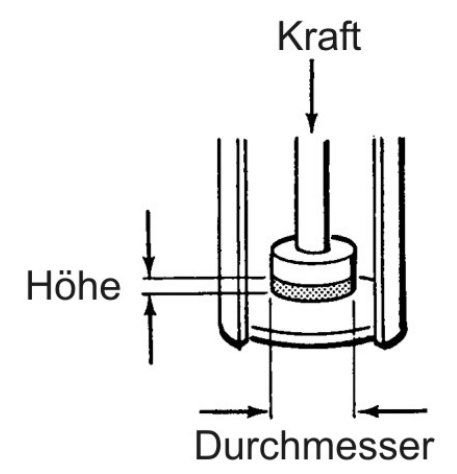

d)

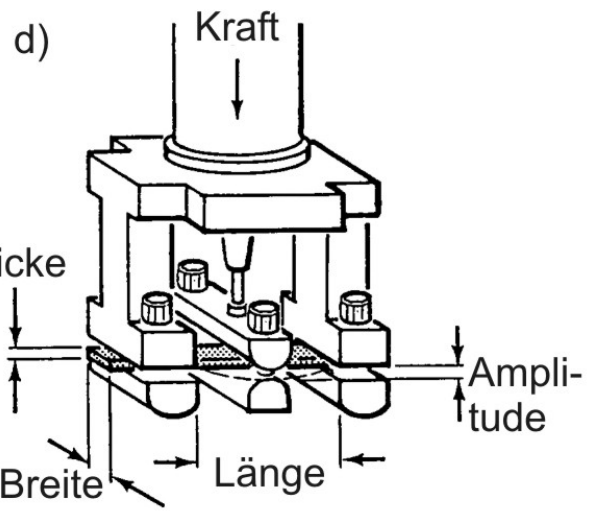

Abbildung 3.9: $\quad$ Schematische Darstellung der Messmodi: a) Uniaxiale Dehnung, b) Kompression, c) Biegebalken mit einseitiger und d) beidseitiger Fixierung. ${ }^{[24]}$

\subsubsection{Präparation von zylindrischen Proben}

Für die mechanische Spektroskopie von EMAA-Proben ist es nötig, diese in eine wohl definierte Probenform zu überführen. Eine praktische Geometrie für die Messung der 


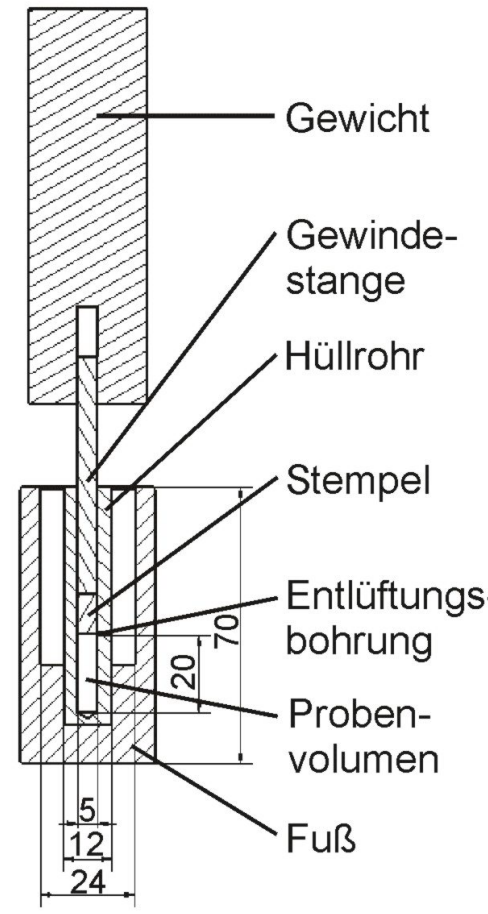

Abbildung 3.10: EMAA

Probenpresse $/ \mathrm{mm}^{[25]}$

elastischen Eigenschaften von Polymerproben in einem Temperaturbereich von der Flüssig-Stickstofftemperatur bis zum Einsetzen des viskosen Fliesens ist die eines Zylinders. ${ }^{[26]}$ Um diese frei von Blasen herzustellen erfolgt das Heißpressen unter Vakuum bei Drücken kleiner als $5 \cdot 10^{-5}$ mbar und etwa $120^{\circ} \mathrm{C}$ in einer eigens dafür hergestellten Probenpresse. Dazu wird das zerkleinerte Probenmaterial in ein Teflon (PTFE)-Hüllrohr gefüllt und dieses mit einem Stempel aus PTFE verschlossen (siehe Abbildung 3.10). Das Hüllrohr wird von einem in das evakuierbare Ofenrohr passenden Fuß aufgenommen und über eine Gewindestange mit einem Stahlgewicht belastet, das eine Kraft von 19.4 N ausübt. Die in den Hohlräumen verbliebene Luft kann beim Evakuieren über eine Entlüftungsbohrung entweichen. Nach Erreichen des Vakuum-Enddrucks wird die Probe 2 Stunden lang aufgeheizt, wobei sich der Stempel senkt und überschüssiges Polymer durch die Entlüftungsbohrung presst. Ist diese verschlossen, stoppt im Regelfall die Senkbewegung. Diese kann auch durch eine Gewindestange mit variabler Länge eingestellt werden. Die entstandene Probe von etwa $20 \mathrm{~mm}$ Länge und $5 \mathrm{~mm}$ Durchmesser kann nun in der Drehmaschine aus dem Hüllrohr geschält werden. Bei sehr weichen Proben empfiehlt es sich, Hüllrohr und Probe zuvor in flüssigem Stickstoff zu kühlen. Aufgrund der Herstellungsverfahren der Teflonzylinder ist es unmöglich eine planparallele Auflagefläche der Polymerproben zu schaffen. Die Enden der Probenzylinder müssen somit mittels Skalpell nachgearbeitet werden. Als effizienteste Lösung eignet sich hier das Einspannen in eine Drehmaschine und das Schneiden unter hoher Rotationsgeschwindigkeit. Auch hier muss gegebenenfalls mit flüssigem Stickstoff gekühlt werden. Falls in der Mitte der Auflagefläche eine kleine Erhöhung zurück bleibt, so ist diese anschließend unbedingt zu entfernen, da ansonsten der Zylinder nicht vollständig in der Halterung aufliegen kann. Die fertigen Probenzylinder werden zum Schutz vor Wasser in einem Exsikkator bei Zimmertemperatur aufbewahrt. 


\subsubsection{Dielektrische Spektroskopie}

Die dielektrische Spektroskopie (DES) bietet eine zur mechanischen Spektroskopie alternative Methode der Messung der mechanischen Eigenschaften und der Dynamik in einem nicht leitenden System. Die Durchführung dieser Messungen erfolgt in Zusammenarbeit mit dem Arbeitskreis von Herrn Dr. Lunkenheimer am Institut für Experimentalphysik V in Augsburg.

Die prinzipielle Funktionsweise ist dabei wie folgt. ${ }^{[27]}$ Eine Probe wird in ein elektrisches Wechselfeld gebracht und dann die Polarisation des Materials als Funktion der Frequenz gemessen. Elektrisches Feld $E$ und Polarisation $P$ sind außer Phase, wenn Relaxationen im Material stattfinden. Eine Änderung des dielektrischen Verlusts, $\varepsilon^{\prime \prime}$, deutet auf Relaxationen im Material hin.

Ein schematisiertes Beispiel für ein Relaxationsspektrum zeigt Abbildung 3.11.

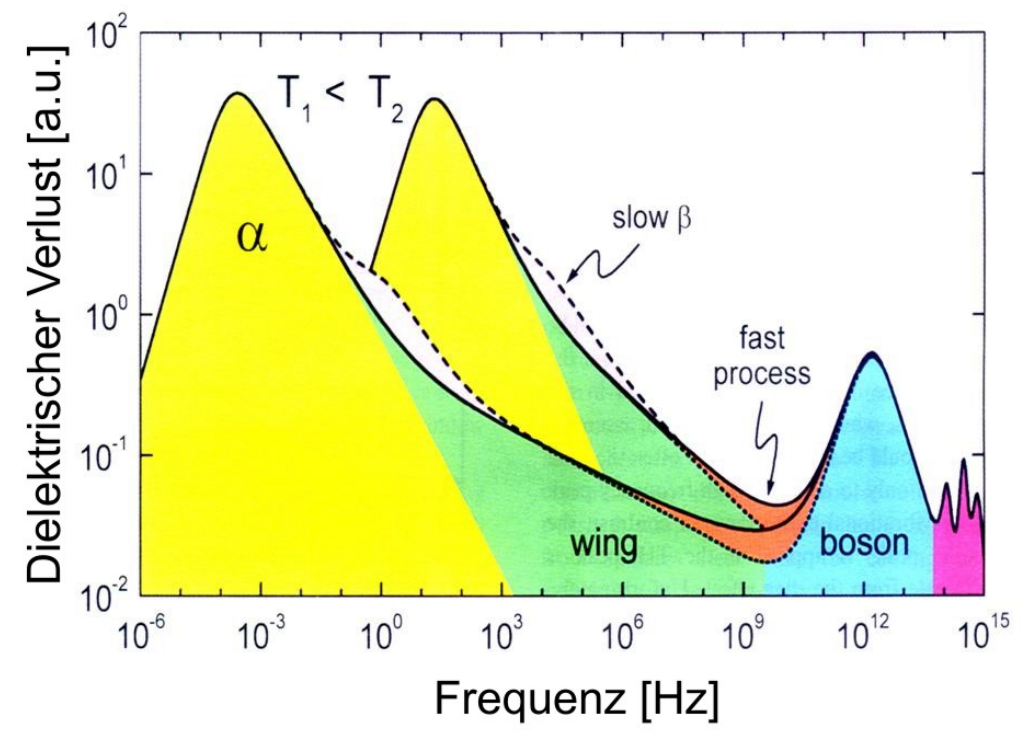

Abbildung 3.11: Lunkenheimer-Loidl-Plot ${ }^{[28]}$ : Schematische Darstellung des Relaxationsspektrum eines amorphen Systems in Form einer schematischen Messung des dielektrischen Verlusts bei zwei Temperaturen als Funktion der Frequenz, die dabei 21 Dekaden überspannt. Die einzelnen Relaxationen sind dabei unterschiedlich eingefärbt.

Da die dynamischen Prozesse auf einander aufbauen, ist es sinnvoll das Spektrum von rechts nach links zu beschreiben.

Oberhalb $10^{14} \mathrm{~Hz}$ wird die Dynamik durch Molkülschwingungen im Infrarotbereich bestimmt. ${ }^{[29]}$ Unterhalb, bis etwa $10^{10} \mathrm{~Hz}$, wird sie durch "Gitter"-Schwingungen 
dominiert. Diese kooperative Bewegung führt zum so genannten "Boson-Peak" im Verlustspektrum. ${ }^{[30,31]}$ Darunter beobachtet man ein Minimum, gefolgt von einem nahezu konstanten Bereich („Nearly Constant Loss“ (NCL)). Das kann im Rahmen der Modenkopplungstheorie erklärt werden. ${ }^{[32]}$ Bei noch tieferen Frequenzen kommt man in den Bereich des "Wing", wobei nicht klar ist, ob es sich um eine Flanke oder ein Maximum handelt, das von der angrenzenden $\alpha$-Relaxation überlagert wird. ${ }^{[33]}$ Bei einigen Glasformern wird in diesem Bereich dieses Maximum beobachtet, die so genannte langsame $\beta$-Relaxation. ${ }^{[34]}$ Wie stark $\alpha$ - und $\beta$-Relaxation einander überdecken hängt dabei stark von der Temperatur bzw. Frequenz ab. ${ }^{[35,36]}$ Die beiden Prozesse sind bei tiefen Temperaturen, bzw. niedrigen Frequenzen besser getrennt. Die $\alpha$-Relaxation stellt schließlich den Übergang ins viskose Fließen dar, wobei die Probe makroskopisch deformiert wird.

Die in den DMA-Untersuchungen gemachten Erkenntnisse könnten sich auch in DESRelaxationsspektren zeigen. Hier sollte eine zusätzliche Relaxation bei etwas höheren Frequenzen als der $\alpha$-Übergang für unstatistische Proben zu beobachten sein.

\subsubsection{Bestimmung der Molmassenverteilung}

Die Bestimmung der Molmassenverteilung für die Ethen-Methacrylsäurecopolymere erfolgt mit Hilfe der Gel-Permeations-Chromatographie (GPC). In der Arbeitsgruppe steht hierfür eine GPC-Anlage bestehend aus einem Waters Inline-Degasser, einer Waters 515 Doppelkolbenpumpe, einem JASCO AS-2055 Autosampler und einem Waters 2410 Differential Refraktometer zur Verfügung. Eine Vorsäule und drei Trennsäulen der Firma PSS werden verwendet.

(1) PSS SDV, $8 \times 50 \mathrm{~mm}, 5 \mu$

(2) PSS SDV, $8 \times 300 \mathrm{~mm}, 5 \mu, 10^{5} \AA$

(3) PSS SDV, $8 \times 300 \mathrm{~mm}, 5 \mu, 10^{3} \AA$

(4) PSS SDV, $8 \times 300 \mathrm{~mm}, 5 \mu, 10^{2} \AA$

Die Arbeitstemperatur der Anlage liegt bei $35^{\circ} \mathrm{C}$. Als Eluent wird Tetrahydrofuran (THF) bei einer Flussgeschwindigkeit von $1.0 \mathrm{ml} \cdot \mathrm{min}^{-1}$ verwendet. Als interner Standard dient Toluol. Eine Kalibrierung erfolgt mit eng verteilten Polystyrol (PS)-Standards ( $M=410$ bis $\left.2 \cdot 10^{6} \mathrm{~g} \cdot \mathrm{mol}^{-1}\right)$. Die erhaltenen Daten werden mit der Software "PSS WinGPC" für 
Windows weiterverarbeitet. Aufgrund des hohen unpolaren Ethenanteils in den Copolymerproben sind nicht alle Proben unter diesen Bedingungen löslich.

Die Bestimmung der Molmassenverteilungen der unlöslichen Proben erfolgte über eine Hochtemperatur-GPC in Zusammenarbeit mit der Arbeitsgruppe von Herrn Prof. Busch am Institut für Technische und Makromolekulare Chemie in Darmstadt. Hier wird eine Waters Hochtemperatur GPC 150-C plus mit RI-Detektor bei einer Mess-Temperatur von $140{ }^{\circ} \mathrm{C}$ verwendet und besteht ebenfalls aus einer Vor- und drei Hauptsäulen.

(1) PSS Polefin, $8 \times 50 \mathrm{~mm}, 10 \mu$

(2) PSS Polefin, $8 \times 300 \mathrm{~mm}, 10^{6} \AA$

(3) PSS Polefin, $8 \times 300 \mathrm{~mm}, 10^{4} \AA$

(4) PSS Polefin, $8 \times 300 \mathrm{~mm}, 10^{3} \AA$

Als Eluent dient hier 1,2,4-Trichlorbenzol (TCB) bei einer Flussrate von $0.95 \mathrm{ml} \cdot \mathrm{min}^{-1}$. Das Injektionsvolumen beträgt $200 \mu \mathrm{l}$. Als interner Standard findet o-Xylol zur Korrektur der Flussrate Verwendung. Zur Kalibrierung können Polyethylen der Firma PSS Polymer Standards Service und Polystyrolstandards der Firma Polymer Laborities Ltd benutzt werden.

Die Proben müssen vor der Messung verestert werden, um die Polarität der Proben zu reduzieren. Dazu werden die Copolymere in $25 \mathrm{ml}$ Standgläser mit Normschliff eingewogen. Nach Zugabe von $20 \mathrm{ml}$ TCB werden die Proben ca. zwei Stunden im Wärmeschrank bei $140{ }^{\circ} \mathrm{C}$ temperiert. Anschließend wird die berechnete Menge Silazan zugegeben und mindestens eine weitere Stunde bei $140{ }^{\circ} \mathrm{C}$ temperiert. Während dieser Phase ist es ratsam, die Proben zweimal umzuschwenken, aber nicht zu schütteln. Die heißen Proben werden danach durch einen $1 \mu \mathrm{m}$ Teflonmembranfilter in $4 \mathrm{ml}$ Autosamplergläser filtriert. Die Filtrationseinheit wird beheizt.

\subsubsection{Gepulste Laserdeposition}

Die gepulste Laserdeposition (Pulsed Laser Deposition, PLD) ist eine Methode zur Herstellung sehr dünner Filme und wurde in Zusammenarbeit mit dem Arbeitskreis von Prof. H.-U. Krebs am Institut für Materialphysik in Göttingen durchgeführt. Interessant für die in dieser Arbeit hergestellten Filme ist eine anschließende Analyse der Depositionsprodukte, um eventuelle Einflüsse der Synthesebedingungen auch nach einer Deposition zu beobachten. Für die Deposition wird ein Strahl eines gepulsten Lasers (KrF Excimer-Laser (Lambda Physik LPX 110i) mit 248 nm Wellenlänge und einer Pulsdauer 
(FWHM) von $30 \mathrm{~ns}$ ) mit hoher Energie durch ein Fenster in eine Vakuumkammer mit einem Druck von weniger als $5 \cdot 10^{-8}$ mbar geleitet und dort auf ein Target, welches aus dem zu deponierenden Material besteht, fokussiert. Dieses wird durch die Energie des Lasers (ca. $60 \mathrm{~mJ} \cdot \mathrm{Puls}^{-1}$ ) erhitzt und teilweise abgetragen. Die bestrahlte Fläche hat eine ungefähre Größe von $2 \mathrm{~mm}^{2}$. Das abgetragene Material schlägt sich auf dem einige Zentimeter entfernt gegenüberliegenden Substrat, einem polierten (111)-Siliziumeinkristall, nieder und bildet dort einen Film. Aufgrund der komplizierten Wechselwirkungen des UV-Lichts mit dem Polymer ist die Deposition von Polymeren immer noch Gegenstand von Diskussionen. ${ }^{[37]}$ Eine detaillierte Beschreibung des Prozesses am Beispiel von PMMA findet sich bei ${ }^{[38]}$.

Die Herstellung der Polymertargets erfolgte mit einer beheizbaren Presse. Die Bedingungen sind so zu wählen, dass das Polymer für ca. eine Stunde oberhalb seiner Glastemperatur bei $3 \cdot 10^{7} \mathrm{~N} \cdot \mathrm{cm}^{-2}$ gepresst wird. Als Temperatur wurde üblicherweise $100{ }^{\circ} \mathrm{C}$ gewählt. Auf diese Weise ergeben sich klare, feste Targets.

\subsubsection{Rasterelektronenmikroskopie}

Die Oberfläche der deponierten Polymerfilme wurde mit Hilfe eines Rasterelektronenmikroskops vom Typ Cambridge Instruments S360 (W-Kathode, 20 kV, Sekundärelektronendetektor) in Zusammenarbeit mit der Arbeitsgruppe von Herrn Prof. Krebs am Institut für Materialwissenschaften Göttingen untersucht. Grundzüge der Rasterelektronenmikroskopie (REM) sind zum Beispiel bei Lüth ${ }^{[39]}$ beschrieben. Um starke Aufladungserscheinungen der elektrisch isolierenden Polymerschichten zu vermeiden, wurden die Proben mit einer 15 bis $20 \mathrm{~nm}$ dicken Goldschicht überzogen.

\subsection{8 $\quad{ }^{1} \mathrm{H}$ - und ${ }^{13} \mathrm{C}$-NMR-Spektroskopie in fester Phase}

Die NMR Spektroskopie dient dazu, die chemische Zusammensetzung der Copolymere zu bestimmen. Informationen über die Mikrostruktur der Polymere lassen sich durch quantitative ${ }^{13} \mathrm{C}-\mathrm{NMR}$ Spektroskopie erzielen. Weitere Experimente, darunter auch mehrdimensionale, tragen zum tieferen Verständnis der Kopplungsmuster im Copolymeren bei. Alle Experimente wurden am Max Planck Institut für Biophysikalische Chemie in Göttingen in Zusammenarbeit mit der Arbeitsgruppe von Herrn Dr. Baldus durchgeführt. 
Die in dieser Arbeit synthetisierten Proben lösen sich zum größten Teil nicht bei Zimmertemperatur in Standardlösungsmitteln, die in der Flüssigphasen-NMRSpektroskopie verwendet werden, wie zum Beispiel Trichlormethan $\mathrm{d}^{1}\left(\mathrm{CDCl}_{3}\right)$ oder Tetrachlorethan $\mathrm{d}^{2}\left(\mathrm{C}_{2} \mathrm{D}_{2} \mathrm{Cl}_{4}\right)$. Aus diesem Grund müssen die Copolymerproben bei einer Temperatur oberhalb $120{ }^{\circ} \mathrm{C}$ in Lösung gebracht werden und auch bei mindestens dieser Temperatur gemessen werden. Um eine quantitative Aussage treffen zu können, werden aufgrund der hohen Scananzahl ca. 9 Stunden Messzeit benötigt. Eine vielversprechende und gerade für schwerlösliche Polymere gut anzuwendende Technik bietet hier die Festkörper-NMR-Spektroskopie. Die Probe wird hier ohne spezielle Vorbehandlung vermessen. Lediglich das Einfüllen in einen Rotor, der für das Spektrometer geeignet sein muss, ist notwendig.

\subsubsection{Aufbau des NMR-Spektometers}

${ }^{13} \mathrm{C}$-NMR-Spektren wurden auf einem Bruker Avance $400 \mathrm{MHz}$ wide-bore-Spektrometer mit $2.5 \mathrm{~mm}$ triple-resonance MAS-Probenkopf aufgenommen. Bei dem Experiment handelt es sich um eine H-C CP Kreuzpolarisierung ${ }^{[40]}$, die bei $+5{ }^{\circ} \mathrm{C}$ durchgeführt wird. Die Probe rotiert unter einem Winkel von $54.74^{\circ}$ („Magic Angle Spinning“, MAS) ${ }^{[41]}$ bei einer Drehfrequenz von $20 \mathrm{kHz}$. Es werden jeweils 5120 Scans mit einer Wartezeit zwischen den Scans (Delay) von $2 \mathrm{~s}$ aufgenommen. Als durchschnittliche Messzeit ergeben sich somit ca. drei Stunden. Die ${ }^{1} \mathrm{H}$-Feldstärke bei einem $90^{\circ}$-Puls beträgt $83 \mathrm{kHz}$. Bei der Kreuzpolarisierung wurde die ${ }^{1} \mathrm{H}$-Feldstärke von 68 bis $54 \mathrm{kHz}$ variiert. Die ${ }^{13} \mathrm{C}$ Feldstärke für die Kreuz-polarisierung ist $33 \mathrm{kHz}$. Eine ${ }^{1} \mathrm{H}$-Entkopplung während der Akquisition (Akquisitionszeit $10.3 \mathrm{~ms}$ ) wurde mit der SPINAL64-Sequenz ${ }^{[42]}$ bei ca. $79 \mathrm{kHz}$ durchgeführt.

Für die zweidimensionalen ${ }^{1} \mathrm{H}-{ }^{1} \mathrm{H}$-Doppelquanten Spektren findet ein Bruker Avance II $800 \mathrm{MHz}$ standard-bore-Spektrometer mit triple-resonance $3.2 \mathrm{~mm}$ MAS-Probenkopf Verwendung. Das bei $0{ }^{\circ} \mathrm{C}$ aufgenommene 2D (2Q, 1Q)-Korrelationsspektrum beruht auf der von Hohwy et al. entworfenen SPC5-Sequenz ${ }^{[43]}$ zur Anregung von ${ }^{1} \mathrm{H}-{ }^{1} \mathrm{H}-$ Doppelquantenkohärenz. Der Rotor wird mit einer Drehfrequenz von 20 kHz MAS rotiert. Die ${ }^{1} \mathrm{H}$-Feldstärke ist $100 \mathrm{kHz}$. Die SPC5-Anregung und -Rekonversionszeit beträgt jeweils $100 \mu \mathrm{s}$. Es werden 32 Scans pro $t_{1}$-Inkrement bei einer Wartezeit zwischen den Scans von $2 \mathrm{~s}$ aufgenommen. Die spektrale Weite in $\mathrm{t}_{1}$ beträgt $41 \mathrm{ppm}$, die Akquisitionszeit 
in $t_{2} 25.2 \mathrm{~ms}$. Insgesamt werden $50 \mathrm{t}_{1}$ Experimente aufgenommen, was $\mathrm{zu}$ einer durchschnittlichen Messzeit von einer Stunde führt.

\subsubsection{Bestimmung der Mikrostruktur}

Die quantitative ${ }^{13} \mathrm{C}-\mathrm{NMR}$-Spektroskopie eignet sich zur Bestimmung der chemischen Zusammensetzung. Es ist darauf zu achten, das eine Pulsfolge verwendet wird, die nicht auf dem Transfer der Magnetisierung von leicht anzuregenden Protonen auf die Kohlenstoffatome beruht, da so die Integrale nicht mehr in Relation zum molaren Gehalt zu setzen sind. Liegt ein unterschiedlicher Protonierungsgrad der ${ }^{13} \mathrm{C}$-Kerne vor, kommt es zu einer unterschiedlich starken Magnetisierung. Ein Kohlenstoffatom einer Carbonylgruppe erfährt zum Beispiel eine andere Magnetisierung wie ein Kohlenstoffatom einer Methylengruppe, wenn die Magnetisierung von den Protonen übertragen wird. Da die Experimente, die auf einer direkten Anregung der ${ }^{13} \mathrm{C}$-Atome beruhen sehr zeitintensiv sind und nicht verstärkt zur Strukturaufklärung beitragen würden, ist auf diese Experimente verzichtet worden.

Es wurden Experimente durchgeführt, bei denen durch eine Kreuzpolarisierung maximale Übertragung der Magnetisierung von Protonen zu Kohlenstoffatomen gegeben ist. Die Anregung von Protonen ist aufgrund des höheren gyromagnetischen Verhältnisses und der Häufigkeit der NMR-aktiven Kerne um ein Vielfaches intensiver als bei Kohlenstoffkernen.

Das Ziel dieser Experimente ist es, mittels einer bei van Boxtel ${ }^{[44]}$ aufgestellten Signalzuordnung des ${ }^{13} \mathrm{C}-\mathrm{NMR}$ Spektrums für das Copolymer Poly(Ethen-coMethylacrylat) (EMA) Information über die Nachbarschaft einer Säuregruppe im Copolymer zu erhalten. 


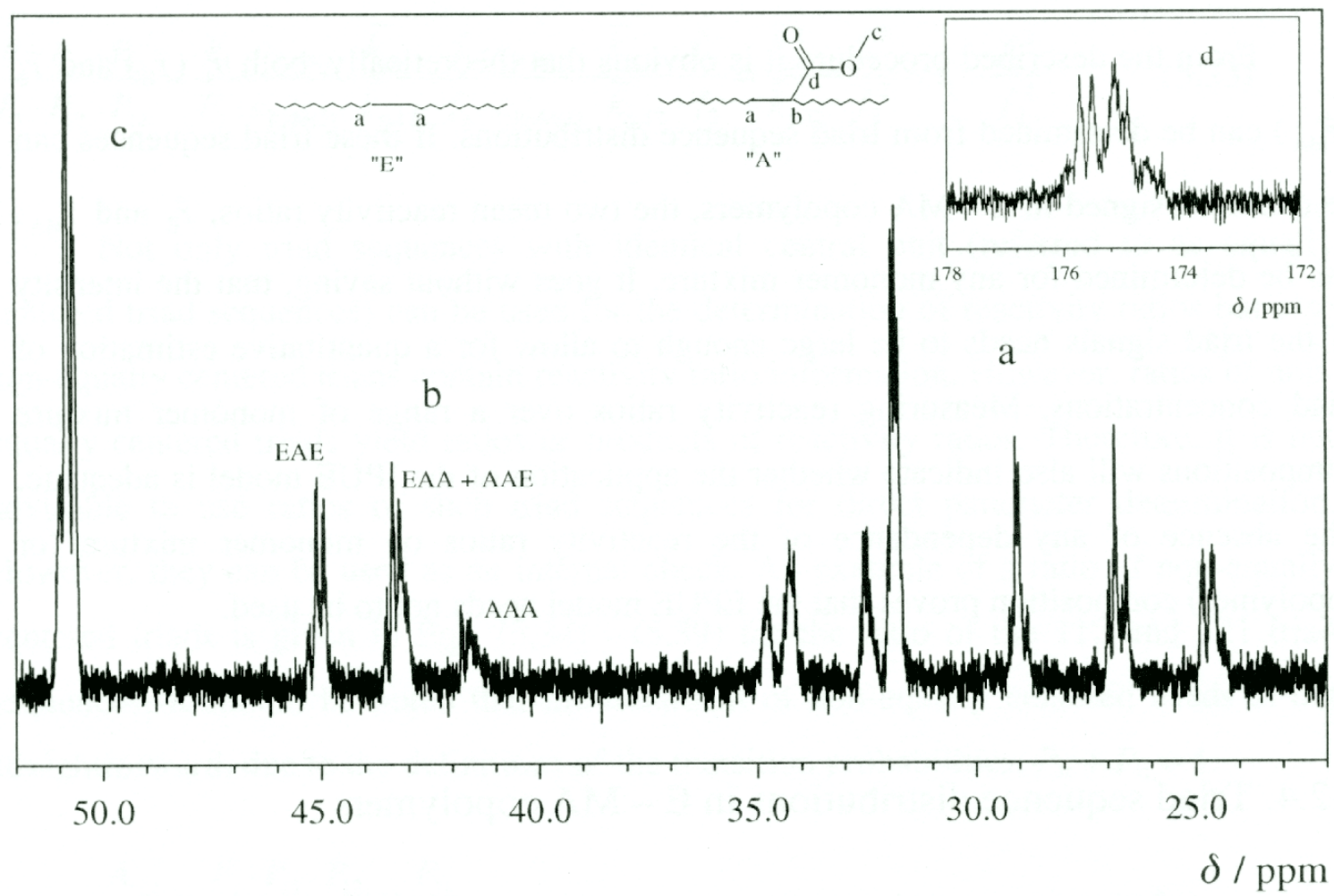

Abbildung 3.12: ${ }^{13}$ C-NMR-Spektrum (Flüssigphase) eines EMA Copolymers $\left(F_{M A}=0.54\right)$ referenziert auf Tetrachlorethan $d_{2}$ bei 74 ppm. ${ }^{[44]}$

Van Boxtel führte die Untersuchungen für das System Poly(Ethen-co-Methacrylat) durch. Da es sich bei dem Methylacrylat um die methylveresterte Form der Acrylsäure handelt, sind die Einwirkungen der Esterseitenkette auf das C-Atom der Hauptkette zu vernachlässigen. Für EMAA ist an diesem C-Atom eine Methylgruppe vorhanden, die zu einer Veränderung der chemischen Verschiebung beitragen kann. Ein weiterer Unterschied besteht in der Durchführung der Messung. Das literaturbekannte Spektrum wurde mittels Flüssigphasen NMR aufgenommen, die in dieser Arbeit verwendeten Spektren sind, wie bereits erwähnt, ausschließlich Festkörper-NMR-Aufnahmen. Dieser Unterschied könnte theoretisch zu einer Signalverschiebung führen, ändert aber nicht die Relationen der Signale. Das bedeutet ein durch Elektronen weniger vom äußeren Feld abgeschirmterer Kern weißt eine höhere Resonanzfrequenz auf. Das entsprechende Signal erscheint also bei einer höheren chemischen Verschiebung $\delta(\mathrm{ppm})$, im Vergleich $\mathrm{zu}$ einem weniger abgeschirmten Kern.

Abbildung 3.12 zeigt ein Spektrum für EMA, was als Modellsystem herangezogen werden kann. Das Signal bei 45 ppm wird einer isolierten Methacrylsäuremethylestereinheit zu geordnet. Kommen nun weitere Säureeinheiten in der Nachbarschaft hinzu, so führt dieses zu einer zusätzlichen Abschirmung des quartären Kohlenstoffkern und die entsprechende 
chemische Verschiebung ändert sich zu höherem Feld. In der Flüssigphasen-NMR kann sogar zwischen zwei und drei zusammenhängenden Säuregruppen unterschieden werden. Für die in dieser Arbeit verwendeten Copolymere, die in fester Phase analysiert werden, lässt sich ableiten, dass eine isolierte Säureeinheit im Bereich von ca. 45 ppm ein Signal zeigen sollte und dass Säuregruppen, die dem Einfluss einer benachbarten Säuregruppe unterliegen sich zu höherem Feld verschieben.

Bei der radikalischen Polymerisation von Ethen kommt es bekannter Weise zu Verzweigungen durch verschiedene Transferreaktionen. ${ }^{[45]}$ Diese Verzweigungen führen zu einem tertiären Kohlenstoffatom. Durch eine sehr große Linienbreite der FestkörperNMR-Spektren ist ein möglicher Einfluss der Verzweigungen zu berücksichtigen. Aus der Literatur ist bekannt, dass zum Beispiel bei Butylacrylat ${ }^{[9]}$ (Abbildung 3.13) das Signal für verzweigte Kohlenstoffatome im Bereich von 47 bis 48 ppm liegt. In Arbeiten über Methylacrylat ${ }^{[46]}$ wurde ebenfalls ein Signal bei 48 ppm einem quartären Kohlenstoffatom zugeordnet.

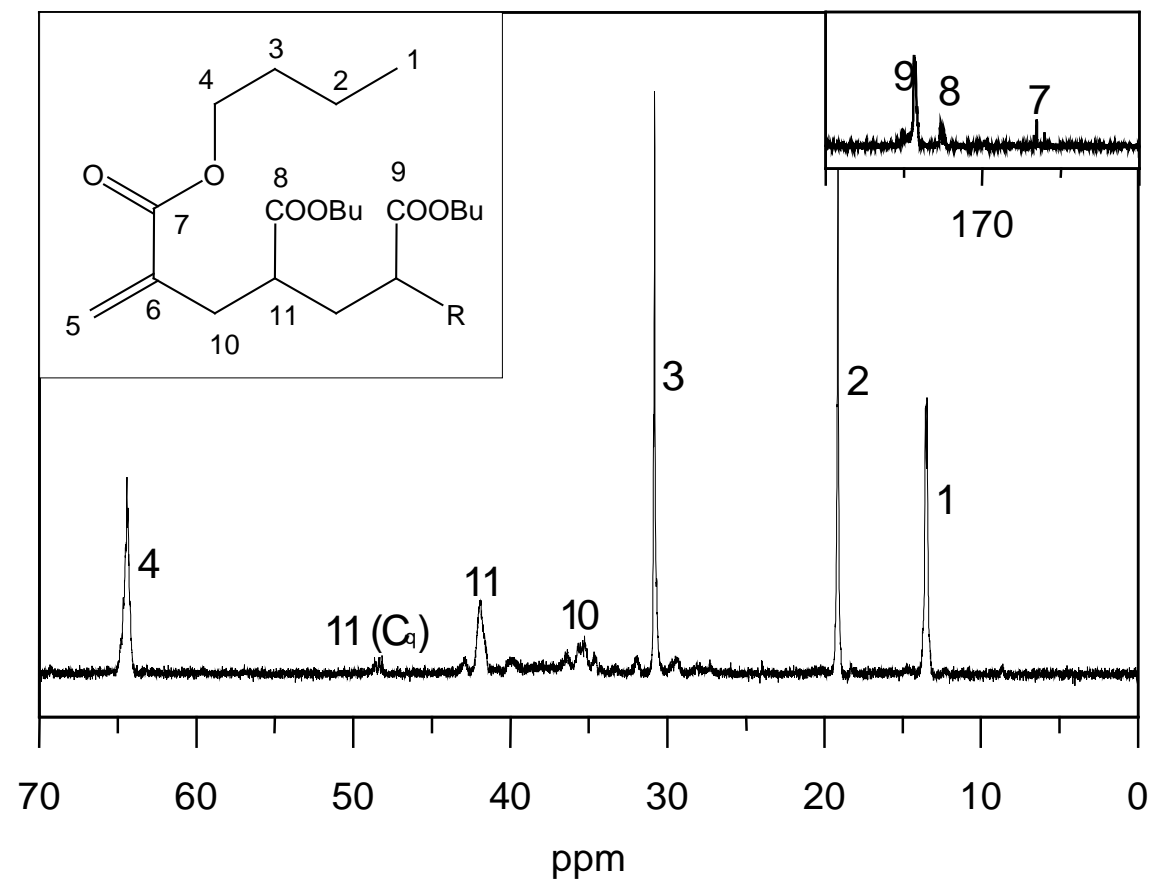

Abbildung 3.13: ${ }^{13}$ C-NMR-Spektrum (Flüssigphase) eines Butylacrylat-Homopolymer. ${ }^{[9]}$

Aufgrund der bei der Methacrylsäure vorhandenen Methylgruppe am $\alpha$-C-Atom kann es hier nicht zu einer Verzweigung kommen, da kein Wasserstoff in dieser Position vorhanden ist. Allerdings sind Transferreaktion für die Hauptkette (backbone), bestehend aus Ethyleneinheiten, zu berïcksichtigen. Hier können durch inter- und intramolekularen Transfer Verzweigungen entstehen. Einen Anhaltspunkt für eine resultierende chemische 
Verschiebung für Polyethylen liefert ${ }^{[47]}$. Dort wird der verzweigten CH-Gruppe eine Verschiebung von $41 \mathrm{ppm}$ zugeordnet. Für das Copolymer ist nicht auszuschließen, dass eine Verzweigung eine chemische Verschiebung zeigt, die in den Bereich des erwarteten Signals für die konjugierten Säuren hineinfällt.

Eine ausführliche Diskussion der Ergebnisse findet sich in Kapitel 4.13.1.

Für eine genauere Aufklärung der Struktur wurde zweidimensionale ${ }^{1} \mathrm{H}-{ }^{1} \mathrm{H}$-Spektroskopie durchgeführt. Durch das oben beschriebene Experiment lässt sich nachvollziehen, welche Säureprotonen eine Kopplung mit anderen Säureprotonen aufweisen. Aus diesem Resultat lässt sich abschätzen, ob eine nicht-statistische aufgebaute Probe mehr Kopplungen aufweist als eine Statistische. Es lässt sich aus den Spektren jedoch nicht ableiten, ob es sich um inter- oder intramolekulare Wasserstoffbrückenbindungen handelt. Unstatistische Proben sollten aber eine höhere Intensität dieses aus der Kopplung hervorgehenden Kreuzsignals besitzen. Bei benachbarten Säuregruppen ermöglicht die räumliche Anordnung die Ausbildung von intramolekularen Wasserstoffbrückenbindungen. Intermolekulare Wechselwírkungen hingegen benötigen eine räumliche Nähe der Säuregruppen. Das bedeutet, dass die Polymerkette dementsprechend ausgerichtet sein muss, damit eine Kopplung stattfinden kann.

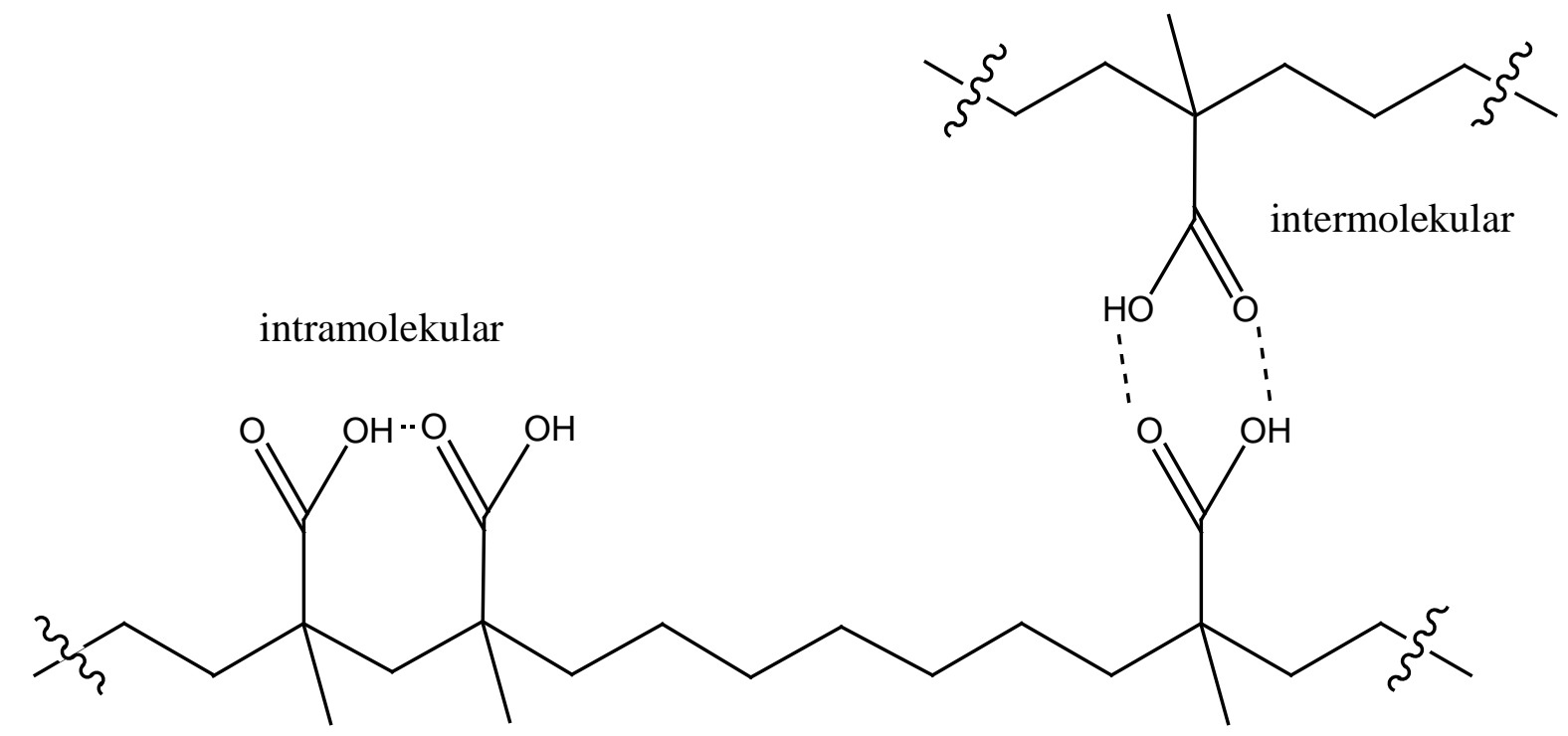

Abbildung 3.14 Schematische Darstellung von intra- und intermolekularen Wasserstoffbrückenbindungen. 


\subsubsection{Bestimmung der Mikrohärte}

In Zusammenarbeit mit dem Institut für Mikrotechnik in Hannover wurde eine Härtemessung an den Copolymerproben durchgeführt. Hierbei wurde nach dem Vickersverfahren vorgegangen ${ }^{[48]}$ und eine Berkovicspitze (dreiseitige Pyramide) verwendet. Die Messungen wurden auf einem Triboscope Nanoindentor von Hysitron ausgeführt. Die Messungen wurden mit einer maximalen Kraft von $100 \mathrm{mN}$ zur Aufnahme von Krafteindringkurven bei $20^{\circ} \mathrm{C}$ durchgeführt. Aus den Krafteindringkurven lässt sich die Mikrohärte bestimmen.

\subsubsection{Thermogravimetrische Analyse}

Die Thermogravimetrische Analyse (TGA) dient zur Bestimmung von Massenänderungen einer Probe. Die Messungen wurden im Arbeitskreis von Herrn Prof. Schmidt am Institut für Makromolekulare Chemie in Bayreuth auf einer TGA/SDTA 851e (Mettler Toledo) ausgeführt. Die Heizrate beträgt $10^{\circ} \mathrm{C} \cdot \mathrm{min}^{-1}$. Die Probe wird in einen Tiegel aus feuerfestem, inertem Material gebracht, zum Beispiel Platin, und gewogen. Der Probentiegel ist an eine Mikrowaage gekoppelt. Somit lässt sich die Masseänderung der Probe während einer Temperaturvariation detektieren. Der gesamte Probenraum wird zur Vermeidung von Oxidationsprozessen mit einem Schutzgas (Stickstoff, $50 \mathrm{ml} \cdot \mathrm{min}^{-1}$ ) gespült. Eventuelle Zersetzungen des Materials können somit eindeutig über einen Massenverlust der Probe nachgewiesen werden. Moderne Geräte werden in Verbindung mit einem Massenspektrometer betrieben. Eine detaillierte Analyse der austretenden Gase, wie zum Beispiel $\mathrm{CO}_{2}$, ist somit möglich.

Dieses Verfahren eignet sich besonders gut um eine eventuelle Zersetzung von Polymerproben zu beobachten. Bei der Probenpräparation für die DMA-Analysen konnte bereits bei Temperaturen von schätzungsweise $200{ }^{\circ} \mathrm{C}$ eine Verfärbung der Proben beobachtet werden. Einige Proben zeigten bei dieser Temperaturbehandlung eine leichte Gelbfärbung. Eine anschließende Malträtierung der Proben bei noch höheren Temperaturen führte zu einer Braunfärbung. Diese Färbung könnte auf eine Zersetzung der Probe oder eine Oxidation hinweisen. Die Ergebnisse der stichprobenartig vermessenen Copolymere (siehe Kapitel 4.5) lassen aber den Schluss zu, dass es unter Schutzatmosphäre bei Temperaturen unterhalb von $300^{\circ} \mathrm{C} \mathrm{zu}$ keiner signifikanten Veränderung des Materials kommt. 


\subsubsection{Reißdehnung}

Die Reißdehnung ist ein häufig verwendeter Materialkennwert. Er gibt an, bis zu welcher Länge bezogen auf die Ausgangslänge des Probenmaterials eine Dehnung möglich ist. Bei Erreichen dieses prozentualen Wertes tritt eine Abriss der Probe ein:

$$
A=\frac{\Delta L}{L_{0}} \cdot 100 \%=\frac{L_{u}-L_{0}}{L_{0}} \cdot 100 \%
$$

wobei $A$ die Reißdehnung, $L_{0}$ die Ausgangslänge, $L_{\mathrm{u}}$ die Länge nach dem Abriss bedeuten. Hohe Werte sprechen somit für ein stabiles Polymer. Das verwendete Universal-Prüfgerät ist hierbei ein Instron 5565 (ebenfalls Arbeitskreis Prof. Schmidt, Bayreuth).

\subsubsection{Optische Messungen an Polymeren}

Es wurden Messungen durchgeführt, um einen Unterschied in den optischen Eigenschaften der Polymerproben zu finden. Dazu wurden drei wichtigste Kenngrößen herangezogen: Transparenz, Trübung (haze) und Klarheit (clarity).

Die Transparenz ist definiert als das Verhältnis von Intensität des eine Probe durchlaufenden Lichtes zu der Intensität des eingestrahlten Lichtes. Proben, die wenig Licht absorbieren sind transparent und Proben, die nicht durchschienen werden können, werden als opak bezeichnet.

Die Trübung ist definiert über folgende Gleichung: ${ }^{[49]}$

$$
\text { haze }=\frac{\text { Intensität des Lichtes, welches mehr als } 2.5^{\circ} \text { gestreut wurde }}{\text { Intensität des gestreuten Lichtes }+ \text { Intensität des durchgetretenen Lichtes }} \cdot 100 \%
$$

Die Klarheit definiert sich über: ${ }^{[49]}$

$$
\text { clarity }=\frac{\text { Intensität des Lichtes, welches weniger als } 0.1^{\circ} \text { gestreut wird }}{\text { Intensität des einfallenden Lichtes }} \cdot 100 \%
$$

Die Probenvorbereitung ist mit größter Sorgfalt durchzuführen, da sich Oberflächeneffekte sehr stark auf die Resultate der optischen Messungen auswirken. Die Proben werden bei einer wohl definierten Temperatur in einem DACA MicroInjector (DACA inc., Goleta, USA) aufgeschmolzen. Für die in dieser Arbeit verwendeten Polymere wurden Temperaturen von 80 bis $175^{\circ} \mathrm{C}$ verwendet. Die Probenvorlage, in die die aufgeschmolzenen Proben gedrückt werden, wurden meistens bei Zimmertemperatur belassen. In einigen Fällen wurde eine Wasserkühlung verwendet um eine Temperatur von 
ca. $15^{\circ} \mathrm{C}$ zu erreichen. Als Probenkörper ergeben sich Plättchen mit einem Durchmesser von $18 \mathrm{~mm}$ für die optischen Messungen. Die Vorlagenform kann ausgetauscht werden, so dass auch andere Formen erhalten werden, die zum Beispiel in Zugversuchen (Reißdehnung) oder in der DMA vermessen werden können.

Die Untersuchung der optischen Eigenschaften wurde im Arbeitskreis von Prof. Schmidt (Bayreuth) auf einem Haze-gard plus der Firma Gardner durchgeführt. 


\subsection{Fehlerbetrachtung}

\subsubsection{Unsicherheit der Messgeräte}

- Die Regelgenauigkeit des eingesetzten Massenflussreglers ist nach Herstellerangabe besser als $\pm 0.02 \mathrm{~kg} \cdot \mathrm{h}^{-1}$ bei einer Reproduzierbarkeit von $1 \%$ des Endwertes. Der Einsatzbereich des Reglers erstreckt sich bis $2 \mathrm{~kg} \cdot \mathrm{h}^{-1}$.

- Der Fehler der zur Druckmessung verwendeten Absolutdruckaufnehmer (HBMMesstechnik, Klasse 0.2) beträgt $\pm 0.2 \%$ des Skalenendwertes von 3000 bar. Die resultierende Unsicherheit beträgt somit \pm 6 bar.

- Die Unsicherheit der Temperaturbestimmung wird vom Hersteller der Thermoelemente mit $\pm 0.25 \mathrm{~K}$ angegeben. Die Genauigkeit der Temperaturbestimmung mit den verwendeten Thermoelementen wurde regelmäßig mit einem Präzisionswiderstandsthermometer (Pt 100, Dostmann, Modell P500) überprüft. Im verwendeten Temperaturbereich betrug die Abweichung maximal 0.5 K. ${ }^{[1]}$

- Die Unsicherheit der IR-spektroskopischen Copolymeranalyse resultiert aus mehreren Ursachen. Bis $\mathrm{zu}$ einer Absorbanz von 1.5 ist ein für quantitative Messungen ausreichend lineares Verhalten des Detektors zu erwarten. Für Werte zwischen 0.3 und 0.8 Absorbanzeinheiten ist der durch Abweichungen von der Linearität verursachte Fehler des Detektors nach Angabe des Herstellers kleiner als $1 \%$. Bei der Integration können zusätzliche Abweichungen durch Ungenauigkeiten in der Bestimmung der Basislinie auftreten. Die Unsicherheit der anhand von IR-Kalibrationen bestimmten Copolymerzusammensetzung für die verschiedenen Copolymersysteme beläuft sich auf kleiner als \pm 3 mol-\%. ${ }^{[11]}$ 


\section{Literaturverzeichnis Kapitel 3}

[1] M. Busch, Dissertation, Göttingen (1993)

[2] M. Buback, M. Busch, K. Lovis, F.-O. Mähling, Chem. Ing. Tech. 67 (1995) 1652

[3] M. Buback, M. Busch, K. Lovis, F.-O. Mähling, Macromol. Chem. Phys. 197 (1996)

[4] K. Lovis, Dissertation, Göttingen (1995)

[5] L. Wittkowski, Dissertation, Göttingen (1998)

[6] F.-O. Mähling, Dissertation, Göttingen (1995)

[7] F. Becker, Dissertation, Göttingen (2006)

[8] M. Buback, H. Lendle, Z. Naturforsch. 34 a (1979) 1482

[9] M. Müller, Dissertation, Göttingen (2005)

[10] M. Buback, H. Latz, Macromol. Chem. Phys. 204 (2003) 638-645

[11] H. Latz, Dissertation, Göttingen (2004)

[12] M. Müller, mündliche Überlieferung

[13] M. Buback, L. Wittkowski, S. A. Lehmann, F.-O. Mähling, Macromol. Chem. Phys. 200 (1999) 1935

[14] M. Buback, L. Wittkowski, Macromol. Chem. Phys. 201 (2000) 419-426

[15] United States Patent 4,248,990

[16] European Patent 0146620B1

[17] E.S. Watson, J. Justin, N. Brenner, M. J. O’Neil, Analytical Chemistry 36 (7) (1964)

[18] M. D. Lechner, E. H. Nordmeier, Nachr. Chem. Tech. Lab. 42 (1994) 6

[19] L. G. Weyer, Appl. Spectroscopy Rev. 21 (1985) 1

[20] H. D. Siesler, Makromol. Chem. Symp. 52 (1991) 113

[21] M. Buback, M. Busch, T. Dröge, F.-O. Mähling, C. Prellberg, Eur. Polym. J. 33 (1997) 375

[22] B. Stuart, "Infrared spectroscopy: Fundamentals and applications", John Wiley \& Sons (2004)

[23] T. Dröge, Dissertation, Göttingen (1997)

[24] Perkin-Elmer, "User Manual 7 Series / Unix DMA 7 / DMA 7e", manual version F, Perkin-Elmer (1994)

[25] J. Hachenberg, Dissertation, Göttingen (2006)

[26] K. P. Menard, "Dynamic mechanical analysis: a practical introduction", CRC Press (1999) 
[27] F. Kremer, A. Schönhals, "Broadband dielectric spectroscopy", Springer (2003)

[28] P. Lunkenheimer, U. Schneider, R. Brand, A. Loidl, Contemp. Phys. 41 (2000) 15

[29] B. Schrader, "Infrared and Raman Spectroscopy: Methods and Applications", VCH (1995)

[30] A. I. Chumakov, I. Sergueev, U. van Bürck, W. Schirmacher, T. Asthalter, R. Rüffer, O. Leupold, W. Petry, Phys. Rev. Lett. 92 (2004) 245508

[31] C. A. Angell, J. Phys.: Cond. Mat. 16 (2004) 5153

[32] W. Götze, Cond. Mat. Phys. 1 (4) (1998) 873

[33] J. C. Dyre, Phys. Rev. E 72 (2005) 011501

[34] A. Kudlik, S. Benkhof, T. Blochowicz, C. Tschirwitz, E. Rössler, J. Mol. Struct. 479 (1999) 201

[35] M. D. Ediger, C. A. Angell, S. R. Nagel, J. Phys. Chem. 100 (1996) 13200

[36] E. J. Donth, “The glass transition - Relaxation dynamics in liquids and disordered materials", Springer Verlag (2001)

[37] Chrisey, D. B.; Piqué, A. ; McGill, R. A.; Horwitz, J. S.; Ringeisen, B. R.;

Bubb, D. M. ; Wu, P. K., Chem. Rev. 103 (2003) 553

[38] T. Scharf, Dissertation, Göttingen (2006)

[39] H. Lüth, "Surfaces and Interfaces of Solid Materials", Springer, Berlin (1995)

[40] A. Pines, J. S. Waugh, M. G. Gibby, J. Chem. Phys. 56 (1972) 1776

[41] E. R. Andrew, A. Bradbury, R. G. Eades, Nature 182 (1958) 1659

[42] B. M. Fung, A. K. Khitrin, K. Ermolaev, J. Magn. Reson. 142 (2000) 97-101

[43] Hohwy et al., J.Chem.Phys 110 (1999) 7983-7992

[44] M. van Boxtel, Dissertation, Göttingen (2000)

[45] M. Busch, Habilitation, Göttingen (2003)

[46] B. Steisel, Diplomarbeit, Göttingen (2004)

[47] Fumitaka Horii, Qingren Zhu, Ryozo Kitamaru, Macromolecules 23 (1990) 977981

[48] DIN EN ISO 6507

[49] Exxon mobil chemical, Technology, Volume No. 2 (2003) 


\section{Untersuchungen an Ethen-Methacrylsäure-Copolymeren}

\subsection{Bestimmung der Copolymerzusammensetzung}

Die Zusammensetzung der Copolymerproben wurde mittels FT-IR-Spektroskopie an Filmen, ATR-FT-IR-Spektroskopie und ${ }^{13}$ C-NMR-Spektroskopie bestimmt. Die Abbildungen 4.1 bis 4.3 zeigen erhaltenen Spektren.

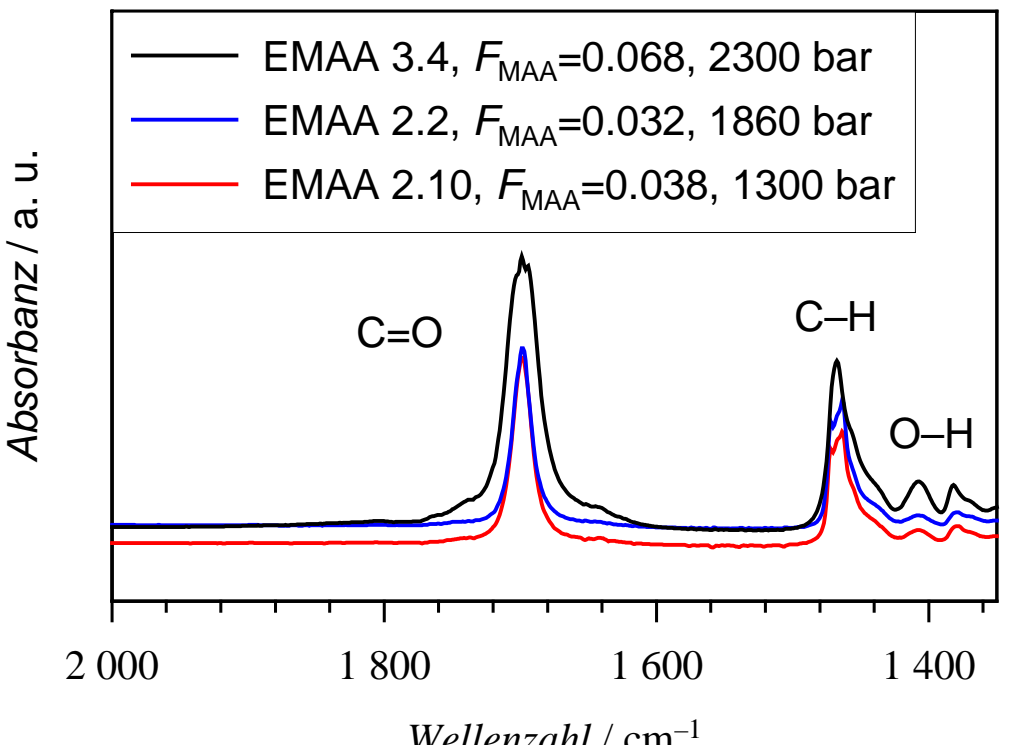

Abbildung 4.1: FT-IR-Absorptionsspektrum dreier EMAA-Filme, die bei verschiedenen Drücken und Säuredosierungen synthetisiert wurden. ${ }^{[1]}$

Zur Bestimmung des Methacrylsäuregehalts der synthetisierten Copolymere steht im Arbeitskreis eine aussagekräftige Analysenmethode zur Verfügung, die FT-IRSpektroskopie. Es besteht die Möglichkeit, in Transmission an gepressten Filmen und der in Kapitel 3.2.2 aufgeführten Gleichung 3.1, den Gehalt mittels entsprechender Integration zu bestimmen. Es ergeben sich Anforderungen an den hergestellten Polymerfilm. Er sollte frei von Lufteinschlüssen sein und muss ausreichend dünn sein, so dass eine Messung der Transmission möglich ist. Um eine ausreichende mechanische Festigkeit zu erhalten, muss eine gewisse Probendicke gegeben sein. Es war nicht möglich, für alle hergestellten Copolymer geeignete Filme herzustellen. Abbildung 4.1 zeigt eine Hoch- (blau) und eine Niederdruckprobe (rot) bei nahezu gleichem Säuregehalt im Copolymer und eine 
Hochdruckprobe (schwarz) mit einem hohem Säuregehalt. Die Lage der Grundlinie ist für die Spektren der Probenreihe EMAA 2, die bei einer Säuredosierung von $34.2 \mathrm{~g} \cdot \mathrm{h}^{-1}$ $\left(f_{\mathrm{MAA}}=0.006\right)$ synthetisiert wurden, verschieden. EMAA 2.10, eine bei $1300 \mathrm{bar}$ hergestellte Niederdruckprobe, weist eine tiefer liegende Grundlinie auf als die entsprechende Hochdruckprobe EMAA 2.2, die bei einem Druck von 1860 bar erhalten wurde. Ansonsten können keine signifikanten Unterschiede im Spektrenverlauf festgestellt werden. Eine davon in erheblichem Maß abweichende Absorbanz liefert die bei einem hohen Säurefluss von $116 \mathrm{~g} \cdot \mathrm{h}^{-1}\left(f_{\mathrm{MAA}}=0.020\right)$ und einem Druck von 2300 bar hergestellte Hochdruckprobe EMAA 3.4. Es ist ein intensiveres Signals im Bereich von $1700 \mathrm{~cm}^{-1}$, welches der Carbonylschwingung zugeordnet werden kann, zu verzeichnen. Ebenfalls einer Intensitätszunahme unterliegen die Signale, bei denen ein Beitrag der $\mathrm{OH}-\mathrm{Gruppe}$ gegeben ist. Das resultierende Verhältnis der integrierten Absorbanzen nach Gleichung 3.1 belegt einen höheren Gehalt an Methacrylsäureeinheiten im Copolymer.

Da aus den aufgeführten Gründen nicht die Möglichkeit bestand, für alle Copolymerproben einen verwendbaren Film zu pressen, wurde auf eine ATR-Einheit zurück gegriffen. Für die Detektion der abgeschwächten Totalreflexion (ATR) ist keine besondere Probenpräparation notwendig. Abbildung 4.2 kann entnommen werden, dass sich die Spektren der Proben EMAA 3.4 und EMAA 3.12 nicht signifikant unterscheiden.

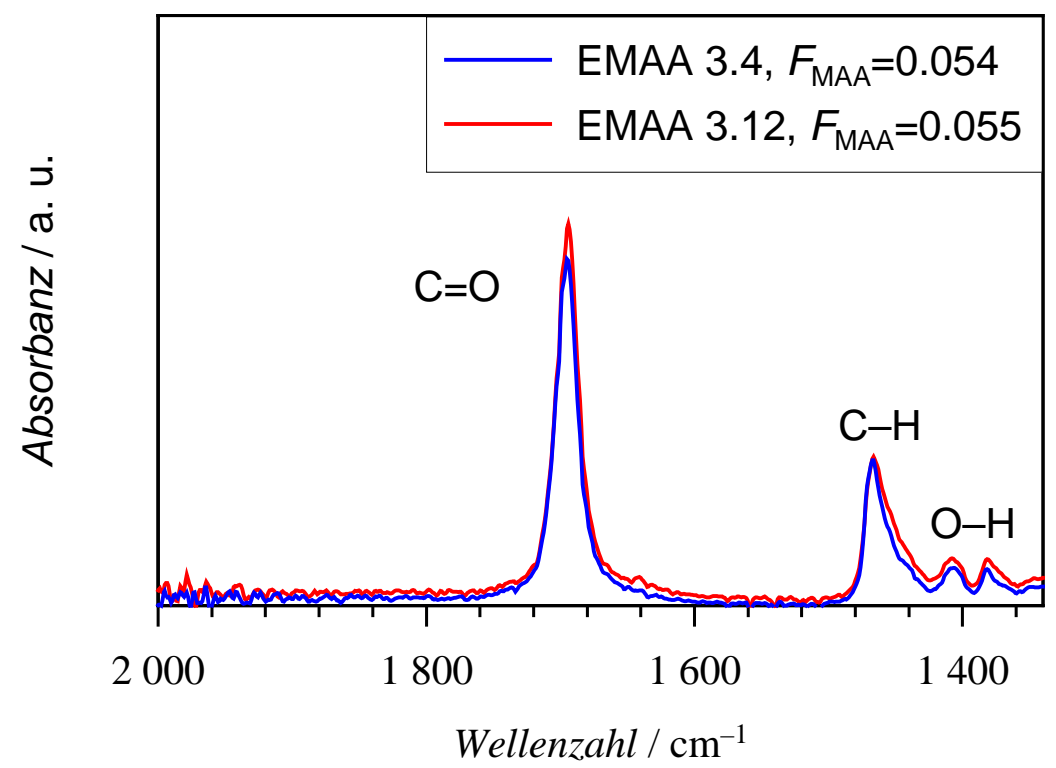

Abbildung 4.2: ATR-FT-IR-Spektrum einer Hochdruckprobe (EMAA 3.4, 2300 bar) und einer Niederdruckprobe (EMAA 3.12, 1300 bar), synthetisiert bei einem hohen Säuregehalt $\left(f_{M A A}=0.020\right)$ im Dosierstrom. ${ }^{[1]}$ 
Der mittels ATR-FT-IR-Spektroskopie bestimmte MAA-Gehalt im Copolymer für die Proben EMAA 3.4 und 3.12 ist nahezu gleich und in Tabelle 4.1 aufgeführt. Mittels dieser Methode konnte für jede Polymerprobe, die in den folgenden Experimenten verwendet wurde, ein Gehalt experimentell bestimmt werden.

Die Bestimmung der Copolymerzusammensetzung ist ebenfalls mit Hilfe der quantitativen ${ }^{13}$ C-NMR-Spektroskopie möglich. Ein Spektrum ist in Abbildung 4.3 gegeben. Die Berechnung des Gehalts beruht auf dem Verhältnis der integrierten Absorbanzen für die Carboxylgruppe im Bereich von $185 \mathrm{ppm}$ zum resultierenden Gesamtintegral aller ${ }^{13}$ C-Signale. Der Nachteil dieser Methode liegt in einer relativ zur FT-IR-Spektroskopie langen Messzeit. Es können keine Experimente durchgeführt werden, bei denen zum Beispiel die Magnetisierung von Protonen auf ${ }^{13} \mathrm{C}$-Kerne transferiert wird. Eine quantitative Auswertung der Signalintegrale wäre dann nicht mehr möglich.

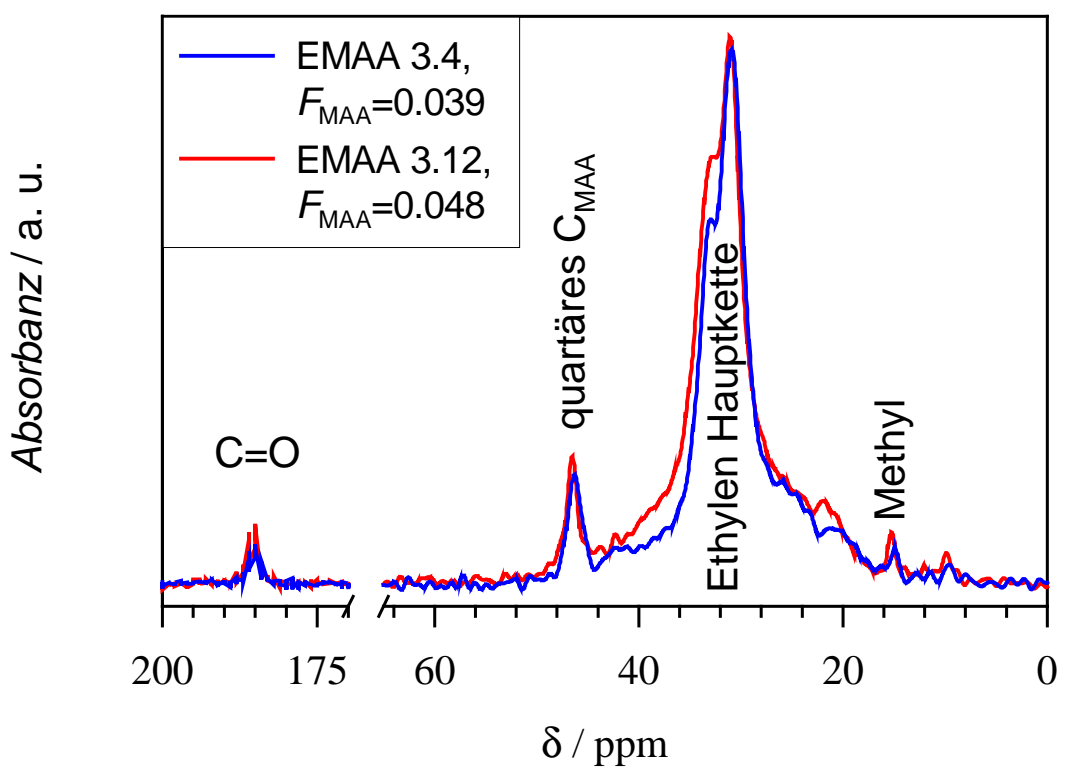

Abbildung 4.3: ${ }^{13}$ C-NMR-Spektren der Hoch- (blau) und Niederdruckprobe (rot) der Probenreihe EMAA 3, synthetisiert bei einem hohen Säuregehalt $\left(f_{M A A}=0.020\right)$ im Dosierstrom. ${ }^{[1]}$

Für alle hergestellten Proben wird eine theoretische Berechnung anhand der Gleichung 4.1 des Methacrylsäureanteils im Copolymer durchgeführt. Hierbei werden die bekannten Copolymerisationsparameter genutzt und eine Umsatzkorrektur der Zusammensetzung in einem iterativen Verfahren gemäß Gleichung 4.1 durchgeführt.

$$
F_{\mathrm{A}}=\frac{r_{\mathrm{A}} f_{\mathrm{A}}^{2}+f_{\mathrm{A}}\left(1-f_{\mathrm{A}}\right)}{r_{\mathrm{A}} f_{\mathrm{A}}^{2}+2 f_{\mathrm{A}}\left(1-f_{\mathrm{A}}\right)+r_{\mathrm{E}}\left(1-f_{\mathrm{A}}\right)^{2}}
$$


Der während der Polymerisation erreichte Umsatz wird in 100 gleichgroße Teilbereiche zerlegt und es wird jeweils ein dazugehöriger Wert für $F_{\text {MAA }}$ berechnet, der im nächsten Rechenschritt als Ausgangswert für eine erneute Berechnung genutzt wird. Der so erhaltene Wert für den jeweiligen Monomerumsatz ist in Abbildung 4.5 beispielhaft für eine Reaktionsreihe (EMAA 3) gegeben, bei der der Zufluss der Methacrylsäure bei $116 \mathrm{~g} \cdot \mathrm{h}^{-1} \mathrm{lag}$, was einem Säuregehalt im Dosierstrom, $f_{\text {MAA, }}$, von 0.020 entspricht. Mehrere Messpunkte für einen Reaktionsdruck ergeben sich aus mehrfach ermittelten Umsätzen für eine Bedingung. Anhand der Beobachtungen während einer Messung (zum Beispiel Temperaturschwankungen) und der sich einstellenden Umsatzkonstanz wurden geeignete Proben für weitere Analysen ausgewählt.

In Abbildung 4.4 sind für eine Probenreihe beispielhaft die Ergebnisse für eine Methacrylsäuregehaltsbestimmung nach 4 verschiedenen Methoden aufgetragen.

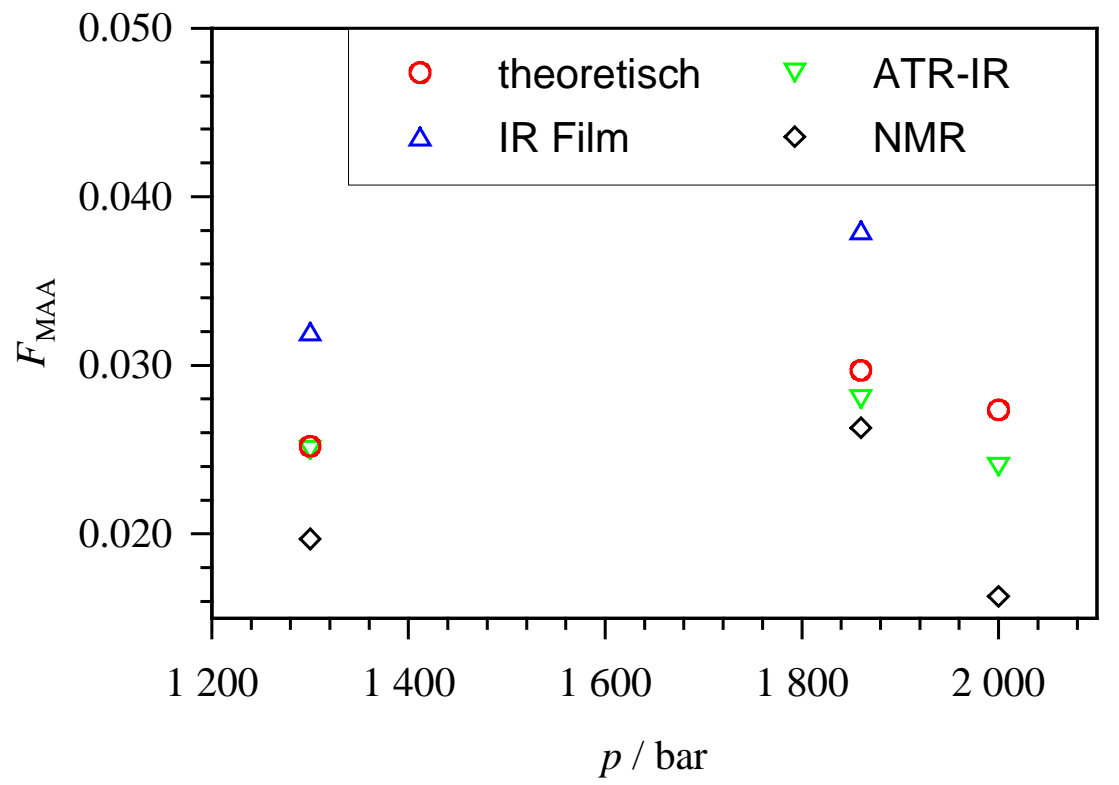

Abbildung 4.4: Gehaltsbestimmung $F_{M A A}$ mittels 4 unterschiedlicher Methoden für die Proben EMAA 1.2 (2000 bar), EMAA 2.2 (1860 bar) und EMAA 2.10 (1300 bar).

Aufgrund der kurzen Messzeit, der einfachen Probenpräparation und der guten Übereinstimmung mit den theoretisch berechneten Daten wird für die folgenden Proben eine MAA-Gehaltsbestimmung experimentell mittels ATR-FT-IR-Spektroskopie vorgenommen. Die Abweichungen der Werte, die mittels IR-Spektroskopie an Filmen entstanden sind, sind auf Blasen in den Filmen zurückzuführen. Die quantitative Auswertung der NMR-Signale kann aufgrund der geringen Relaxationszeiten starke Fehler beinhalten. 


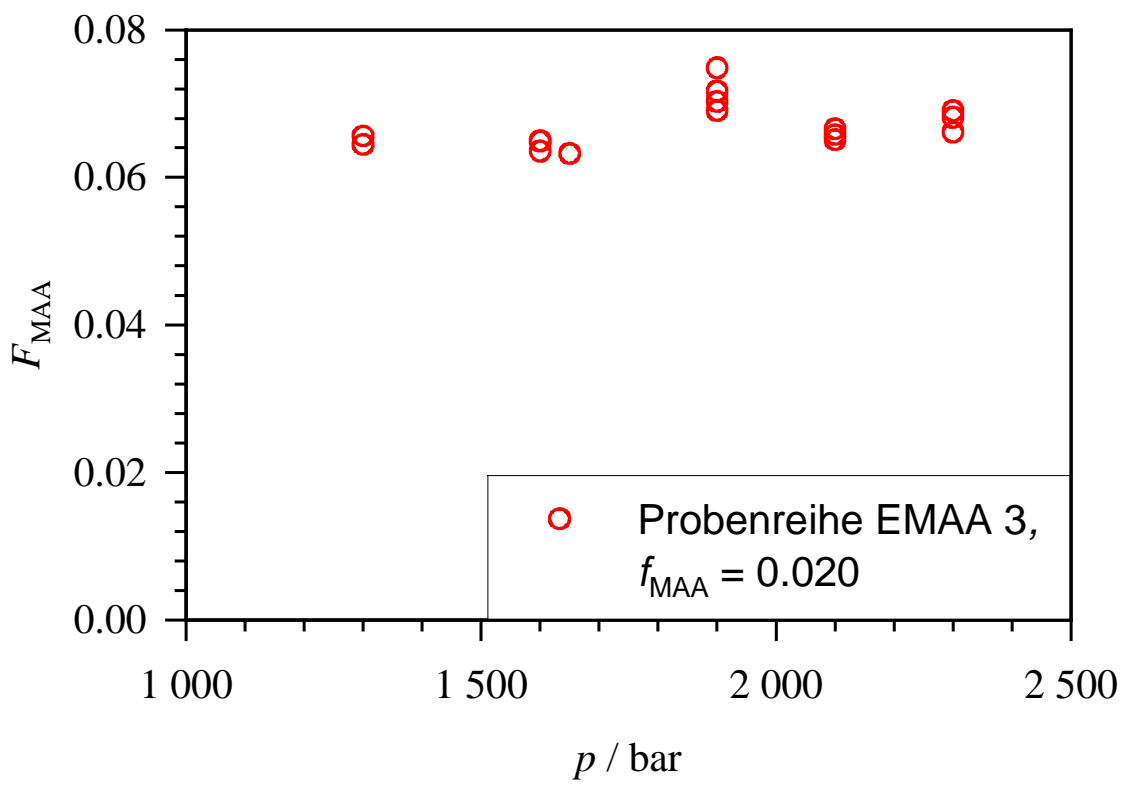

Abbildung 4.5: Theoretisch berechnete Methacrylsäuregehalte für die bei verschiedenen Drücken hergestellten Polymerproben der Versuchsreihe EMAA 3.

Einen Vergleich der mittels ATR-FT-IR-Spektroskopie bestimmten Säuregehalte mit dem theoretisch bestimmten Gehalt für eine durchgeführte Variation des Säuregehalts im Dosierstrom zeigt Abbildung 4.6. Die zwei Messpunkte für ein Verfahren ergeben sich jeweils aus einer Hoch- und einer Niederdruckprobe.

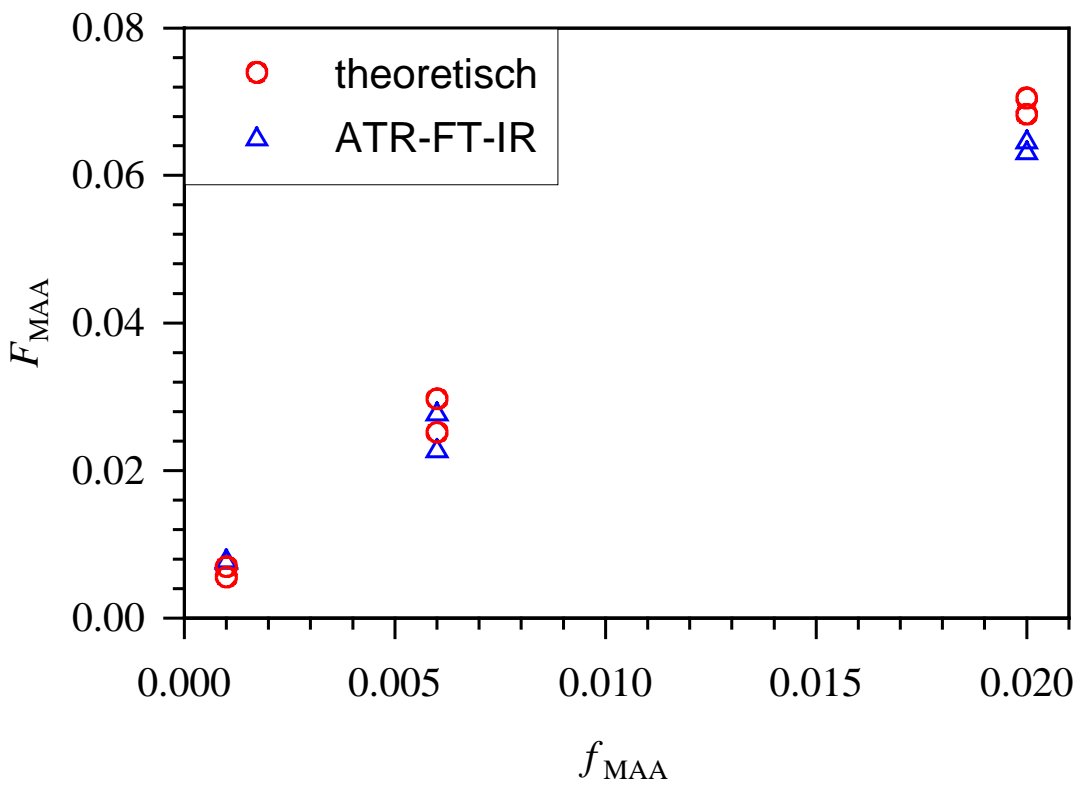

Abbildung 4.6: Vergleich von theoretisch und mittels ATR-FT-IR bestimmten Methacrylsäuregehalten, $F_{M A A}$, in Abhängigkeit vom Methacrylsäuregehalt im Dosierstrom, $f_{M A A}$. 


\begin{tabular}{l|cccccc} 
Probe & $p$ & $T_{\text {Mantel }}$ & $\begin{array}{c}T_{\text {Reaktion }} \\
/{ }^{\circ} \mathrm{C}\end{array}$ & $\begin{array}{c}\text { MAA-Fluss } \\
/ \mathrm{g} \cdot \mathrm{h}^{-1}\end{array}$ & $\begin{array}{c}F_{\text {MAA }} \text { ATR } \\
/ 10^{-2}\end{array}$ & $\begin{array}{c}F_{\text {MAA }} \text { theo } \\
/ 10^{-2}\end{array}$ \\
\hline EMAA 1.2 & 2000 & 242 & 255 & 34.2 & 2.35 & 2.74 \\
EMAA 2.2 & 1860 & 245 & $271-276$ & 34.2 & 2.42 & 2.52 \\
EMAA 2.10 & 1300 & 246 & $268-272$ & 34.2 & 2.64 & 2.97 \\
EMAA 3.4 & 2300 & 244 & 272 & 116 & 5.43 & 6.91 \\
EMAA 3.12 & 1300 & 245 & $268-271$ & 116 & 5.47 & 6.56 \\
EMAA 12.11 & 2300 & 246 & $263-264$ & 5.3 & 0.79 & 0.58 \\
EMAA 12.4 & 1300 & 245 & $268-275$ & 5.3 & 0.81 & 0.55 \\
\hline EMAA 16.2 & 2300 & 240 & $236-238$ & 58 & 7.17 & 8.16 \\
EMAA 16.15 & 1500 & 240 & $238-239$ & 58 & 7.79 & 7.42 \\
EMAA 16.5 & 1300 & 240 & $236-238$ & 58 & 8.73 & 7.93 \\
EMAA 22.9 & 2300 & 260 & $256-261$ & 58 & 6.84 & 6.99 \\
EMAA 22.14 & 1300 & 260 & $258-261$ & 58 & 8.03 & 6.12 \\
EMAA 25.2 & 2300 & 220 & $261-268$ & 58 & 4.31 & 4.07 \\
\hline EMAA 25.6 & 1300 & 220 & $262-268$ & 58 & 5.95 & 5.08 \\
EMAA 27.5 & 2300 & 200 & $236-243$ & 58 & 4.84 & 4.91 \\
EMAA 27.8 & 1400 & 200 & $247-250$ & 58 & 4.18 & 4.47 \\
EMAA 28.2 & 2300 & 250 & $294-300$ & 58 & 2.47 & 3.53 \\
EMAA 28.6 & 1300 & 250 & $293-295$ & 58 & 3.01 & 3.65 \\
EMAA 28.7 & 1000 & 250 & $294-300$ & 58 & 2.96 & 3.62 \\
EMAA 30.3 & 2300 & 170 & $214-243$ & 58 & 6.65 & 6.51 \\
EMAA 31.2 & 2300 & 155 & $215-242$ & 58 & 7.36 & 7.47 \\
EMAA 32.2 & 2300 & 210 & $256-258$ & 58 & 3.33 & 4.24 \\
EMAA 32.3 & 1300 & 210 & $254-258$ & 58 & 5.95 & 6.02 \\
EMAA 33.3 & 2300 & 190 & $230-232$ & 58 & 5.38 & 4.32 \\
EMAA 33.8 & 1400 & 190 & $227-237$ & 58 & 7.99 & 6.24
\end{tabular}

Tabelle 4.1: $\quad$ Syntheseparameter der für weitere Analysen verwendeten Copolymerproben und die Angabe des MAA-Gehalts im Copolymer.

Tabelle 4.1 zeigt eine Übersicht über die für weitere Auswertungen genutzten Polymerproben. Die Copolymere lassen sich aufgrund ihrer Synthesebedingungen in 3 Gruppen einteilen, was durch einen zusätzlichen Trennstrich angedeutet ist. Die erste Gruppe (EMAA 1 bis EMAA 12) weist eine Variation der Säuredosierung auf, was zu einem stark unterschiedlichen Säuregehalt im Copolymer führt. Die zweite Gruppe (EMAA 16 und EMAA 22) sind Proben, die unter adiabatischen Bedingungen hergestellt wurden. Die Manteltemperatur, $T_{\text {Mantel, }}$ ist nahezu gleich der Reaktionstemperatur, $T_{\text {Reaktion}}$. Für die anderen Probenreihen können erhebliche Differenzen zwischen Mantel- und Reaktionstemperatur beobachtet werden. Bei der dritten Gruppe (EMAA 25 bis EMAA 33) wurde eine Variation der Manteltemperatur vorgenommen. Die unterschiedlichen Reaktionstemperaturen können der Tabelle 4.1 entnommen werden. 


\section{Diskussion:}

Aus dem in Tabelle 4.1 resultierenden Unterschied zwischen der theoretischen Berechnung des Säuregehalts, der experimentell ermittelten Werte und der Fluktuation bei den erhaltenen Messwerten bei Wiederholung des ATR-FT-IR-Experiments ergibt sich ein geschätzter Fehler von etwa \pm 0.01 . Die an Folien mittels FT-IR-Messungen bestimmten Konzentrationen sind systematisch höher als die der ATR-FT-IR, welche ihrerseits höher sind, als die durch NMR bestimmten Werte. Prinzipiell lässt sich feststellen, dass die Analysewerte der ATR-FT-IR-Messungen in guter Übereinstimmung mit den theoretisch bestimmten Gehalten stehen. Somit wurden in Tabelle 4.1 für die in dieser Arbeit verwendeten Proben lediglich die ATR-FT-IR-Spektroskopie und die theoretische Berechnung des Säuregehalts zur $F_{\mathrm{MAA}}$-Bestimmung herangezogen. Eine signifikante Abhängigkeit des MAA-Gehalts vom Synthesedruck wird unter Zuhilfenahme aller Messungen an Proben, die in einem weitem Druckbereich hergestellt worden sind, nicht beobachtet. 


\subsection{Bestimmung der Molmassenverteilungen der Copolymerproben}

In diesem Abschnitt werden die Ergebnisse der Bestimmung der Molmassenverteilung für die EMAA-Copolymere vorgestellt. Es wird zunächst der Einfluss des Initiatorflusses auf den Umsatz betrachtet. Um bei einer Niederdruckprobe die gleiche Reaktionstemperatur zu erhalten wie bei einer Hochdruckprobe, ist eine im Vergleich höhere Initiatorkonzentration im Reaktor notwendig (siehe Kapitel 3.1.7.6). Abbildung 4.7 zeigt eine Variation des Methacrylsäuregehalts im Dosierstrom. Die Reaktionstemperatur dieser Proben wurde weitestgehend konstant gehalten und beträgt 265 bis $275^{\circ} \mathrm{C}$. Der Auftragung ist zu entnehmen, dass bei tiefen Drücken jeweils mehr Initiator benötigt wird, um die Temperatur im Intervall von 265 bis $275^{\circ} \mathrm{C}$ zu halten. Der Effekt wird umso deutlicher, je höher die verwendete MAA-Konzentration ist.

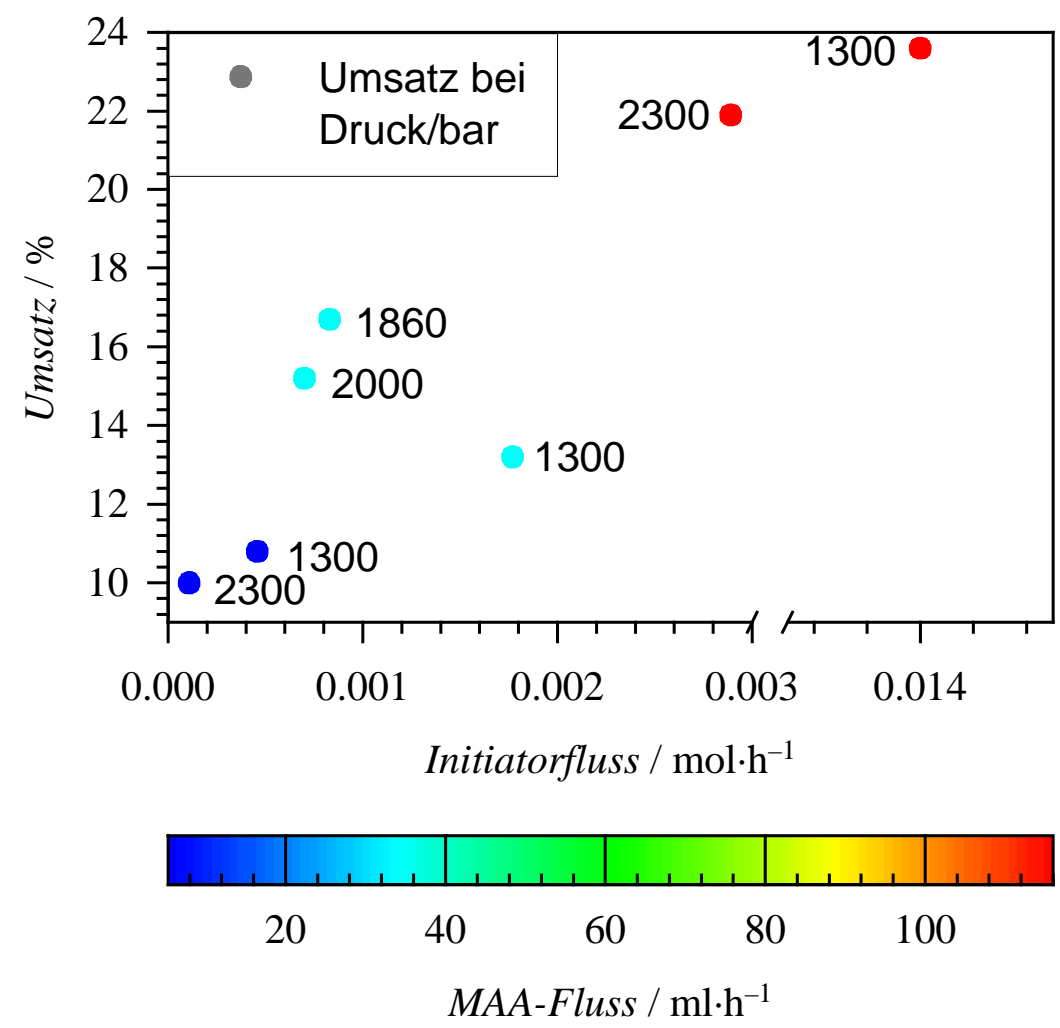

Abbildung 4.7: Gesamtmonomerumsatz bei verschiedenen Initiatordosierungen und Synthesedrücken bei gleicher Synthesetemperatur $\left(265\right.$ bis $\left.275^{\circ} \mathrm{C}\right)$ und einem Ethenfluss von $1800 \mathrm{~g} \cdot \mathrm{h}^{-1}$. 
Abbildung 4.8 zeigt Molmassenverteilungen einer EMAA-Probenreihe, die bei einer Synthesetemperatur im Bereich von 260 bis $275^{\circ} \mathrm{C}$ hergestellt wurde. Der Gehalt an Methacrylsäure im Dosierstrom, $f_{\mathrm{MAA}}$, beträgt 0.006. Dies führt zu einem MAA-Gehalt im Copolymer, $F_{\text {MAA, }}$, von 0.027 bis 0.030 . Es ist deutlich zu erkennen, dass Hochdruckproben eine höhere Molmasse aufweisen als Proben, die bei niedrigerem Druck hergestellt wurden.

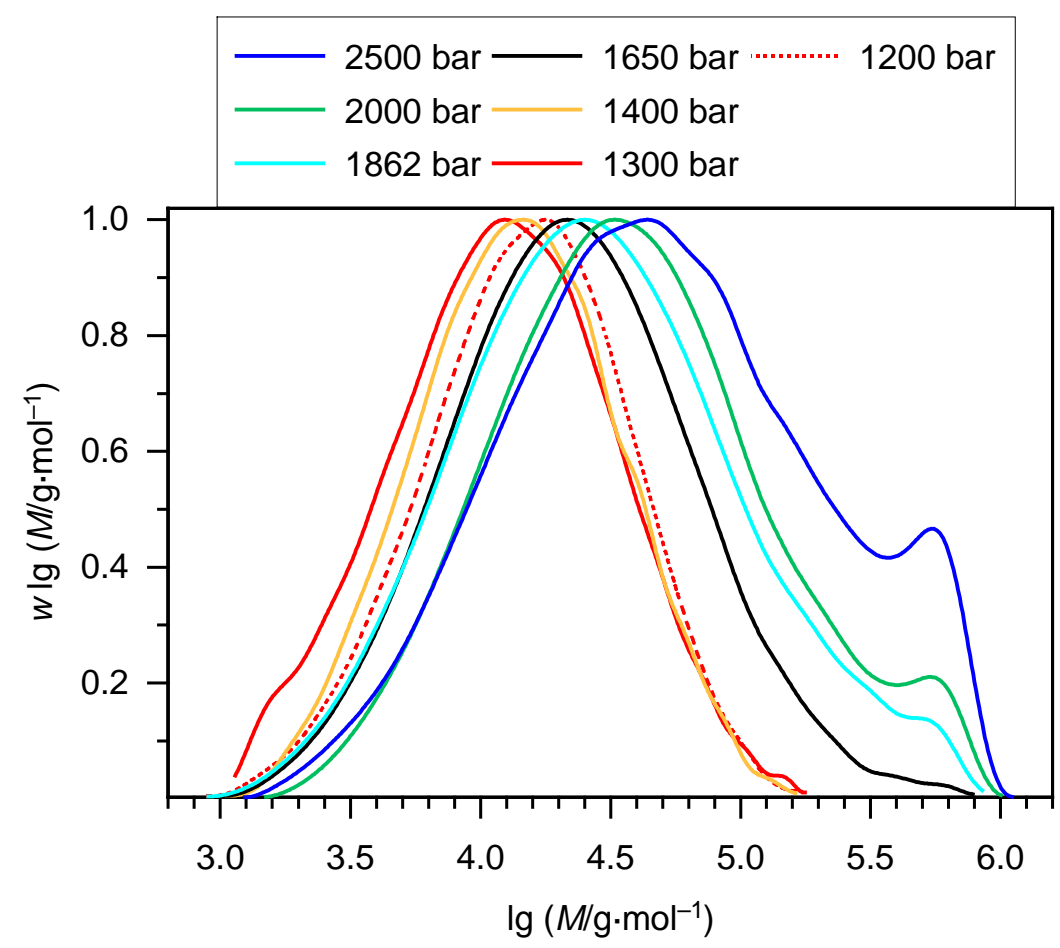

Abbildung 4.8: Molmassenverteilungen von bei 260 bis $275^{\circ} \mathrm{C}$ hergestellten EMAA-

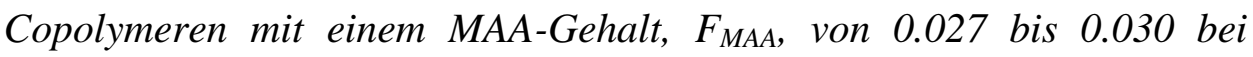
verschiedenen Drücken (Proben gelöst in TCB).

Erhöht sich nun die Initiatorkonzentration im Dosierstrom (siehe auch Abbildung 4.7), so ist eine Verschiebung der Molmassenverteilung hin $\mathrm{zu}$ niedrigeren Molmassen $\mathrm{zu}$ beobachten, da eine höhere Initiatorkonzentration zu kürzeren Polymerketten führt. ${ }^{[2]}$ Dieser Trend lässt sich über den gesamten Druckbereich feststellen, in dem eine homogene Reaktionsführung vorliegt. Bei der vorliegenden Probenreihe wurde zusätzlich zu den unter homogenen Bedingungen hergestellten Proben eine weitere Probe im Bereich der Phasengrenze hergestellt. Es konnte visuell festgestellt werden, dass für diese Bedingungen der „cloud point pressure“ (siehe Kapitel 3.1.3) erreicht wurde und dabei bereits Polymer ausfällt. Hier mussten Druckvariationen im Bereich von 1200 bis 1350 bar vorgenommen werden, um die Reaktion weiterhin kontrollieren zu können. Eine Umsatzkonstanz war 
nicht mehr gegeben und die Reaktionstemperatur fiel teilweise auf unter $240{ }^{\circ} \mathrm{C}$. Diese Probe folgt den oben beschriebenen Tendenzen nicht mehr. Die Molmassenverteilung weist ein Maximum auf, welches zwischen den bei 1400 und 1650 bar hergestellten Proben liegt.

Ein weiterer interessanter Effekt lässt sich bei Molmassenverteilungen von Copolymeren finden, die bei einem Synthesedruck von 1650 bar und höher hergestellt wurden. Hier bildet sich erst ein hochmolekularer Ausläufer, der sich bei höherem Synthesedruck sogar zu einem lokalen Maximum entwickelt. Ein ähnlicher Effekt wurden auch bei anderen Probenreihen für die Hochdruckproben beobachtet. Für diesen hochmolekularen Ausläufer gibt es verschiedene Erklärungsansätze. Zum einen wäre es möglich, dass die verwendete Säulenkombination der GPC Anlage für die Hochtemperaturmessungen bei hochmolekularen Polymeren keine Trennung mehr gewährleisten kann. Tatsächlich wurden für dieses Copolymersystem keine Molmassenverteilungen gefunden, die über eine Molmasse von $10^{6} \mathrm{~g} \cdot \mathrm{mol}^{-1}$ hinaus gingen. Das könnte dafür sprechen, dass die Auflösungsgrenze der Säulen keine weitere Trennung zulässt. Die Polymere lassen sich also ab dieser Molmasse nicht mehr mittels ihres hydrodynamischen Volumens trennen. Es kommt zum direkten Durchlauf von hochmolekularen Polymeren ohne Auftrennung und ein Peak im Bereich dieser Molmassen würde erheblich verstärkt werden. Es werden allerdings auch bei vielen Proben solche hochmolekularen Ausläufer bei niedrigeren Molmassen, im Bereich von $10^{5} \mathrm{~g} \cdot \mathrm{mol}^{-1}$ beobachtet. Es kann geschlossen werden, dass es zwar möglich und aufgrund der beobachteten Form der Molmassenverteilungen auch wahrscheinlich ist, dass die Auflösungsgrenze der Säulen erreicht ist, aber, da der hochmolekulare Ausläufer auch bereits bei tieferen Molmassen vorliegt, die Ausschlussgrenze für hochmolekulare Polymere nicht der alleinige Effekt sein kann. Reaktorfoulingprozesse können $\mathrm{zu}$ Polymeren mit sehr hoher Molmasse führen. Hochmolekulare Polymere fallen an den Reaktorwänden und am Rührwerk aus und werden nach und nach mit den anderen Reaktionsprodukten aus dem Reaktionsraum gespült. Bei den in dieser Arbeit ausgeführten Synthesen spricht allerdings einiges gegen eine Erklärung anhand von Foulingprodukten. Zum einen werden die Hochdruckproben tief im homogenen Bereich des Phasendiagramms hergestellt, das heißt weit entfernt von der Phasengrenze. Sie weisen im Gegensatz zu den Niederdruckproben einen deutlich größeren Abstand zu den für eine einphasige Reaktion notwendigen $p T$-Werten auf. Somit sollten eher Niederdruckproben zum Fouling neigen, was hier allerdings nicht beobachtet wird. Zum anderen sollten Foulingreaktionen eine starke Zeitabhängigkeit aufweisen. Je 
länger der Reaktor betrieben wird, desto mehr Fouling sollte beobachtet werden. Die Hochdruckproben wurden bis auf eine Ausnahme (EMAA 12) stets (zeitlich gesehen) vor den Niederdruckproben synthetisiert. Auch dieses Argument spricht gegen eine Erklärung mittels Foulingprozessen.

Diese zusätzlichen Maxima können durch Copolymere verursacht werden, die während der Synthese Verzweigungsreaktionen unterliegen. Findet ein Transfer zum Polymer statt, so kommt es zu Verzweigungen. Vornehmlich der intermolekulare Transfer führt zu einer Vergrößerung der Molmasse des attackierten Polymers. Diese Transferreaktionen geschehen hauptsächlich bei hohem Druck ${ }^{[3,4]}$ und liefern die plausibelste Erklärung für hochmolekulare Ausläufer.

Für einige Proben war es möglich eine Molmassenverteilung der Polymere, gelöst bei $35^{\circ} \mathrm{C}$ in THF, zu erhalten. Auch diese Proben zeigen einen zusätzlichen hochmolekularen Ausläufer. Die Proben, deren mittels Hochtemperatur-GPC ermittelte Molmassenverteilungen die größten Intensitäten für hochmolekulare Ausläufer aufweisen, sind in THF nicht vollständig löslich. Das kann durch die schlechte Löslichkeit von Polymeren mit einer hohen molekularen Masse erklärt werden und ist in guter Übereinstimmungen mit den Erkenntnissen der Molmassenanalyse mittels GPC. Abbildung 4.9 zeigt, dass mit beiden Aufbauten (Hochtemperatur und Normaltemperatur GPC) hochmolekulare Ausläufer gefunden werden können. Die zusätzlichen Peaks der Molmassenverteilung, die von dem in THF gelöstem Polymer ermittelt wurde, lassen sich auf Oligomere zurückführen. Die Hochtemperatur-GPC-Säule ist in diesem Bereich nicht selektiv genug, um eine derartige Aufspaltung herbei zu führen.

Die erhaltenen Werte für das Zahlenmittel $M_{\mathrm{N}}$ und das Gewichtsmittel $M_{\mathrm{W}}$ der Molmassenverteilungen sind in Tabelle 4.2 aufgetragen. Hieraus kann entnommen werden, dass in allen Fällen eine Kettenlänge von mehr als 100 Monomereinheiten vorhanden ist. Somit kann für eine Segmentbewegung, die bei den mechanischen Analysen gefunden wurde, eine Kettenlängenabhängigkeit weitestgehend ausgeschlossen werden. ${ }^{[13]}$ Bei den Proben EMAA 30.3 (2300 bar) und 31.2 (2300 bar) waren unlösliche Anteile vorhanden. 


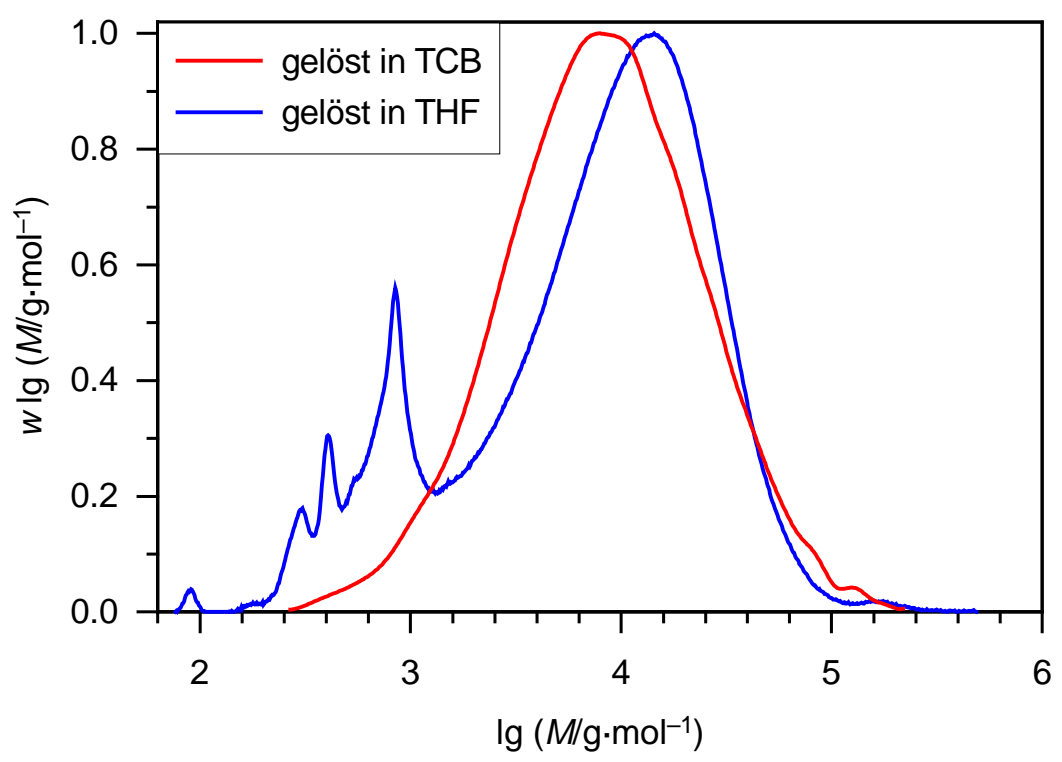

Abbildung 4.9: Vergleich zweier Molmassenverteilungen (in THF bzw. TCB gelöst) eines identischen Copolymers (EMAA 28.6, synthetisiert bei 1300 bar und $295^{\circ} \mathrm{C}, F_{M A A}=0.036$ ).

\begin{tabular}{|c|c|c|c|c|c|c|c|c|}
\hline Probe & $\begin{array}{c}p \\
/ \text { bar }\end{array}$ & $\begin{array}{l}T_{\text {Reak }} \\
{ }^{\circ} \mathrm{C}\end{array}$ & $\begin{array}{c}X \\
1 \%\end{array}$ & $\begin{array}{c}M_{\mathrm{n}} \\
/ 10^{4} \mathrm{~g} \cdot \mathrm{mol}^{-1}\end{array}$ & $\begin{array}{c}M_{\mathrm{w}} \\
/ 10^{4} \mathrm{~g} \cdot \mathrm{mol}^{-1}\end{array}$ & PDI & $\begin{array}{l}F_{\mathrm{MAA}} \\
/ 10^{-2}\end{array}$ & $\begin{array}{c}\text { Kettenlänge } \\
\text { / Monomereinheiten }\end{array}$ \\
\hline EMAA 1.2 & 2000 & 255 & 15.2 & 2.05 & 7.99 & 3.9 & 2.74 & 690 \\
\hline EMAA 2.2 & 1860 & $271-276$ & 16.7 & 1.45 & 6.29 & 4.4 & 2.52 & 490 \\
\hline EMAA 2.10 & 1300 & $268-272$ & 13.2 & 0.75 & 1.90 & 2.5 & 2.97 & 252 \\
\hline EMAA 3.4 & 2300 & 272 & 21.9 & 0.98 & 2.62 & 2.7 & 6.91 & 307 \\
\hline EMAA 3.12 & 1300 & $268-271$ & 23.6 & 0.38 & 0.66 & 1.8 & 6.56 & 118 \\
\hline EMAA 12.11 & 2300 & $263-264$ & 10.0 & 5.37 & 23.00 & 4.3 & 0.58 & 1890 \\
\hline EMAA 12.4 & 1300 & $268-275$ & 10.8 & 1.90 & 9.67 & 5.1 & 0.55 & 670 \\
\hline EMAA 16.2 & 2300 & $236-238$ & 5.6 & 1.96 & 3.74 & 1.9 & 8.16 & 598 \\
\hline EMAA 16.15 & 1500 & $238-239$ & 6.9 & 0.99 & 1.55 & 1.6 & 7.42 & 305 \\
\hline EMAA 16.5 & 1300 & $236-238$ & 6.0 & 0.86 & 1.35 & 1.6 & 7.93 & 264 \\
\hline EMAA 22.9 & 2300 & $256-261$ & 7.2 & 1.33 & 2.99 & 2.3 & 6.99 & 413 \\
\hline EMAA 22.14 & 1300 & $258-261$ & 9.3 & 0.57 & 1.17 & 2.1 & 6.12 & 179 \\
\hline EMAA 25.2 & 2300 & $261-268$ & 17.7 & 1.68 & 8.24 & 4.9 & 4.07 & 552 \\
\hline EMAA 25.6 & 1300 & $262-268$ & 12.1 & 0.68 & 1.64 & 2.4 & 5.08 & 218 \\
\hline EMAA 27.5 & 2300 & $236-243$ & 14.0 & 2.61 & 10.00 & 3.8 & 4.91 & 845 \\
\hline EMAA 27.8 & 1400 & $247-250$ & 16.0 & 0.99 & 3.72 & 3.8 & 4.47 & 322 \\
\hline EMAA 28.2 & 2300 & $294-300$ & 23.6 & 1.17 & 8.11 & 6.9 & 3.53 & 389 \\
\hline EMAA 28.6 & 1300 & $293-295$ & 20.5 & 0.46 & 1.41 & 3.1 & 3.65 & 153 \\
\hline EMAA 28.7 & 1000 & $294-300$ & 20.1 & 0.31 & 0.78 & 2.5 & 3.62 & 102 \\
\hline EMAA 30.3 & 2300 & $214-243$ & 9.7 & 2.22 & 23.26 & 10.5 & 6.51 & 699 \\
\hline EMAA 31.2 & 2300 & $215-242$ & 7.6 & 2.33 & 22.35 & 9.6 & 7.47 & 720 \\
\hline EMAA 32.2 & 2300 & $256-258$ & 17.8 & 1.47 & 8.87 & 6.0 & 4.24 & 482 \\
\hline EMAA 32.3 & 1300 & $254-258$ & 10.4 & 0.60 & 1.51 & 2.5 & 6.02 & 190 \\
\hline EMAA 33.3 & 2300 & $230-232$ & 16.9 & 1.75 & 9.87 & 5.6 & 4.32 & 573 \\
\hline EMAA 33.8 & 1400 & $227-237$ & 98 & 0.72 & 2.33 & 32 & 6.24 & 227 \\
\hline
\end{tabular}

Tabelle 4.2: $\quad$ Zahlen-, Gewichtsmittel, Polydispersitätsindex (PDI) und die Anzahl, der im Copolymer verbauten Monomereinheiten bei einem theoretisch ermitteltem Säuregehalt $F_{\text {MAA. }}$ 
Aus Tabelle 4.2 können auch die Werte für den Polydispersitätsindex (PDI) entnommen werden. Auffällig ist, dass für die hier synthetisierten Polymere, die technischen Produkten entsprechen, teilweise sehr geringe PDI von zum Beispiel 1.6 aufweisen. Diese Werte sind eher bei der kontrollierten radikalischen Polymerisationen zu erwarten. ${ }^{[5]}$ Besonders die unter adiabatischen Bedingungen hergestellten Polymere besitzen eine enge Molmassenverteilung, wie das Beispiel EMAA 16.5 (1300 bar) in Abbildung 4.10 zeigt.

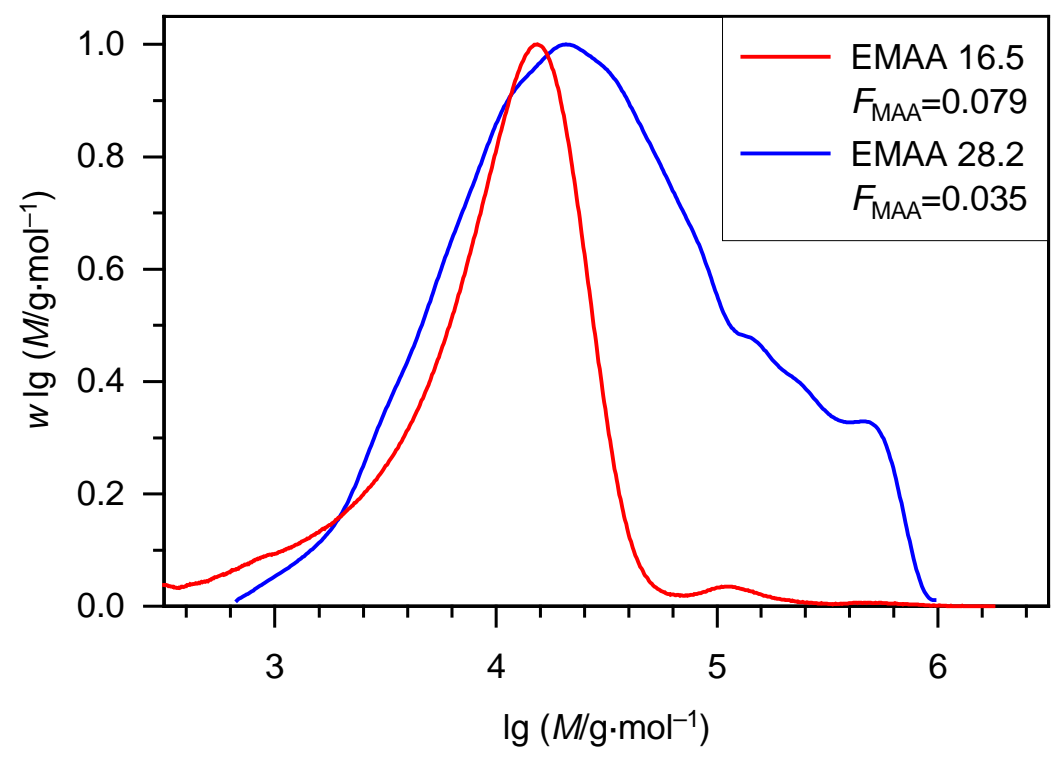

Abbildung 4.10: Molmassenverteilungen für ein unter adiabatischen Bedingungen bei $240{ }^{\circ} \mathrm{C}(P D I=1.6)$ und ein bei $300{ }^{\circ} \mathrm{C}(P D I=6.9)$ synthetisiertes EMAACopolymer.

Kommt es zur Ausbildung von hochmolekularen Ausläufern in der Molmassenverteilung, wie im Falle der Probe EMAA 28.2 (2300 bar), so wird ein hoher PDI ermittelt. Die Molmassenverteilung der Probe EMAA 16.5 (1300 bar) (rot) suggeriert rein optisch eine deutlich engere Verteilung als die Probe EMAA 28.2 (blau).

Um einen eventuellen Einfluss des Säuregehalts im Copolymer zu erfassen, wurde in Abbildung 4.11 der PDI gegen den theoretisch bestimmten Säuregehalt im Copolymer, $F_{\mathrm{MAA}}$, aufgetragen. Es muss berücksichtigt werden, dass die gezeigten Proben bei einem unterschiedlichen Umsatz und verschiedenen Initiatorflüssen synthetisiert wurden. Bei unterschiedlichen Probenreihen lag in vielen Fällen eine andere Temperaturen vor. 


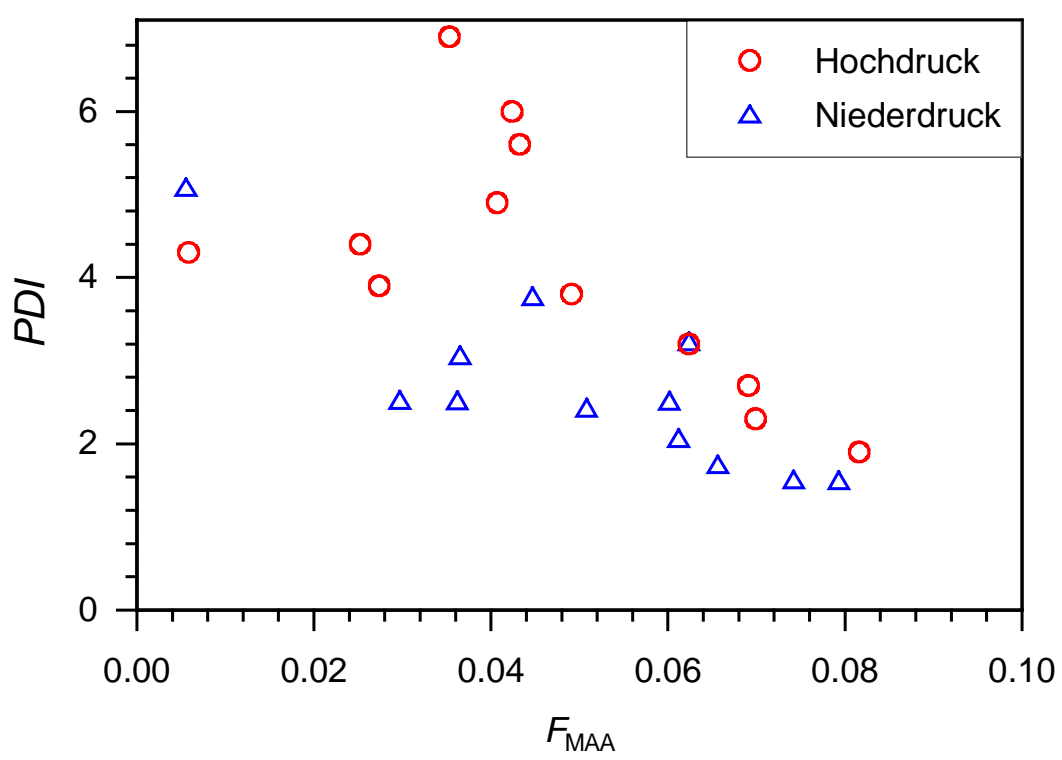

Abbildung 4.11: Polydispersitätsindex in Abhängigkeit von dem theoretisch ermittelten Säuregehalt $F_{M A A}$ im Copolymer unterschieden nach Hoch- und Niederdruckproben. Die Proben EMAA 30.3 (2300 bar) und 31.2 (2300 bar) fanden keine Berücksichtigung, da nichtlösliche Anteile vorhanden waren. (Lösungsmittel TCB).

Für Niederdruckproben liegt im Vergleich zu den Hochdruckproben eine geringere Kettenlänge vor, was auf die erhöhte Initiatorkonzentration zurückgeführt werden kann. Durch die geringere Kettenlänge ergibt sich somit in vielen Fällen für die Niederdruckproben ein kleinerer $P D I$, da eine größere Kettenlänge eine generell breitere Verteilung mit sich bringt, wie in Abbildung 4.10 gezeigt.

Des Weiteren kann Abbildung 4.11 entnommen werden, dass bei einem hohen Säureanteil im Copolymer ein geringerer PDI vorliegt. In diesem Zusammensetzungsbereich sind hauptsächlich Proben zu finden, die unter adiabatischen Bedingungen synthetisiert wurden. Die Minimierung eines Temperaturgradienten im Reaktor, wie es unter adiabatischen Bedingungen der Fall ist, kann somit eventuell zu einer Minimierung des PDI beitragen. Ebenfalls ein Effekt kann von der verwendeten Initiatorkonzentration ausgehen. Bei einem hohen Säureanteil im Dosierstrom wird eine hohe Initiatorkonzentration benötigt, um den gewünschten stabilen Betriebspunkt bei ausreichend hohem Umsatz und Temperatur zu erhalten. Die hohe Initiatorkonzentration führt wiederum zu kürzeren Polymerketten, was zu einer Verringerung des $P D I$ führt.

Für weitere Analysen sollten sich somit gerade die Proben eignen, die für eine Hoch- und für eine Niederdruckprobe nahezu den gleichen PDI aufweisen. EMAA 22.9 (2300 bar) $(P D I=2.3)$ und $22.14(1300 \mathrm{bar})(P D I=2.1)$ besitzen einen nahezu gleichen Wert, der 
zusätzlich für eine schmale Molmassenverteilung spricht. Sollten sich für diese beiden Proben signifikante Unterschiede bei den im folgenden gezeigten Analyseverfahren ergeben, so kann ein Einfluss des PDI weitestgehend ausgeschlossen werden. Um den Ergebnissen vorwegzugreifen, sei hier erwähnt, dass gerade die Probenreihe EMAA 22 sehr deutliche Unterschiede für Hoch- und Niederdruckproben sowohl in den DSCAnalysen als auch besonders in den DMA-Analysen (siehe Abbildung 4.40) zeigt. Somit steht fest, dass auch Proben, die eine nahezu gleichbreite Molmassenverteilung besitzen, sich in ihren Relaxationen erheblich unterscheiden. Das „Chemical Confinement“, beschrieben in Kapitel 5, wird hier beobachtet.

Da die Polydispersität, wie an Probenreihe EMAA 22 bei den jeweiligen Analysemethoden gezeigt, keinen erkennbaren Einfluss auf das „Chemical Confinement“ hat, können auch Probenreihen für weitere Analysen verwendet werden, die einen erheblichen Unterschied in dem PDI für Hoch- und Niederdruckproben aufweisen. DSC-Analysen zum Beispiel konnten für alle in Tabelle 4.2 aufgeführten Proben durchgeführt werden. 


\subsection{Weiterführende ATR-FT-IR-Spektroskopieuntersuchungen}

Wie bereits in Kapitel 4.1 gezeigt, wurde die ATR-FT-IR-Spektroskopie zur Bestimmung des MAA-Gehalts im Copolymer genutzt. Aus der Literatur ist bekannt, das für Carbonsäuredimere eine sehr breite Absorbanz im Wellenzahlenbereich von 2300 bis $3400 \mathrm{~cm}^{-1}$ auftritt. ${ }^{[6]}$ Dabei weist die integrierte Molarabsorptivität der $\mathrm{OH}-$ Streckschwingung für das Dimer einen erheblich höheren Wert auf als für monomere Säure. Für Acrylsäure werden zum Beispiel für monomere Säure eine integrierte Molarabsorptivität von $65 \mathrm{~km} \cdot \mathrm{mol}^{-1}$ und für dimere Acrylsäure $580 \mathrm{~km} \cdot \mathrm{mol}^{-1}$ angegeben, gemessen bei einem Druck von 250 bar, $28{ }^{\circ} \mathrm{C}$ in $\mathrm{CCl}_{4}$ als Lösungsmittel. ${ }^{[6]}$ Die integrierte Molarabsorptivität ist unter den geschilderten Bedingungen ca. 9 mal höher für dimere Acrylsäure. Liegen, wie in Kapitel 5 vermutet, bei der Niederdruckproben aufgrund eines nicht-statistischen Aufbaus im Vergleich zu Hochdruckproben mehr Wasserstoffbrückenbindungen (WBB) vor, so sollten sich die ATR-FT-IR-Spektren von Hoch- und Niederdruckproben unterscheiden.

\subsubsection{Vergleich von ATR-FT-IR-Spektren für Hoch- und Niederdruckproben}

Wie oben bereits beschrieben, ist der Bereich, in dem die OH-Streckschwingung der Säuredimere erwartet wird, von 2300 bis $3400 \mathrm{~cm}^{-1}$. Durch die Ausbildung von WBB kommt es zu einer verstärkten Absorption in diesem Wellenzahlenbereich. Der direkte Vergleich von Hoch- und Niederdruckproben sollte somit Unterschiede im Verlauf des Spektrums aufzeigen. Für die Niederdruckprobe wird im Bereich der Säuredimere eine verstärkte Absorption erwartet. Nicht trivial wird die Auswertung durch das Vorhandensein einer CH-Absorbanz, die hauptsächlich durch den hohen Ethenanteil der Copolymere verursacht wird. Abbildung 4.12 zeigt das erhaltene Spektrum für Polyethylen (Basislinie korrigiert, auf gleiches Niveau wie die entsprechende Basislinie der Copolymere) und für die Copolymere EMAA 2.2 (Hochdruckprobe) und EMAA 2.10 (Niederdruckprobe), die einen nahezu gleichen Gehalt an MAA-Einheiten im Copolymer aufweisen. 


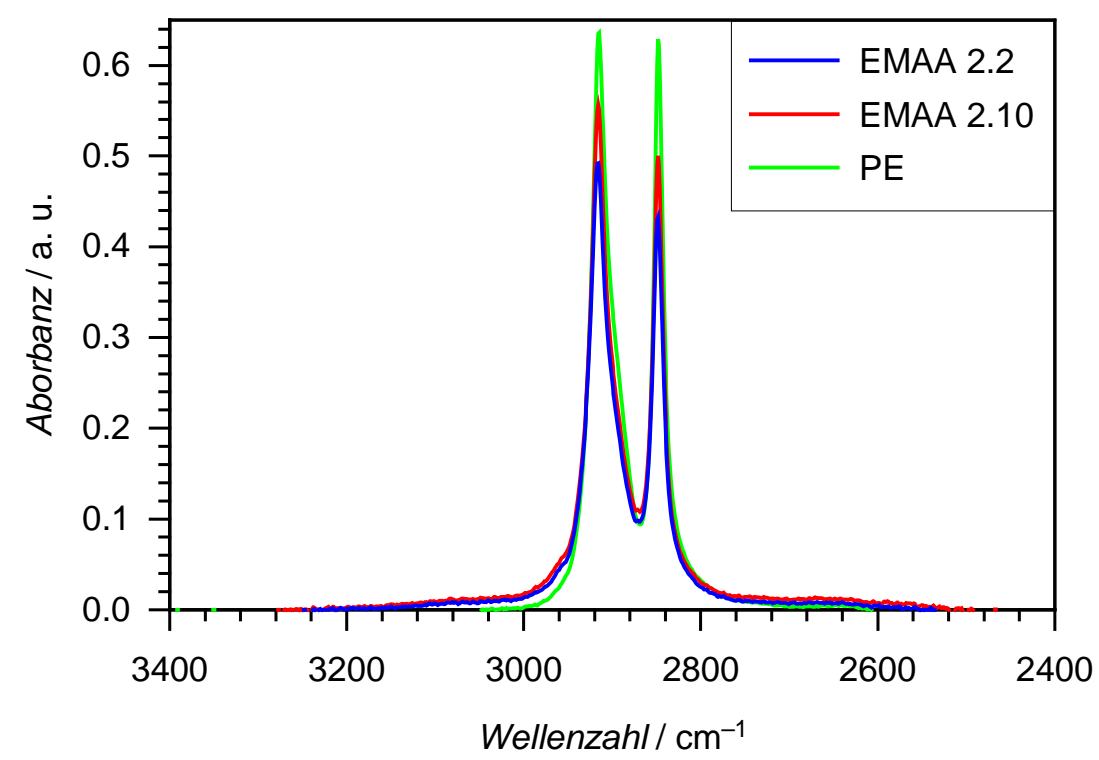

Abbildung 4.12: ATR-IR-Spektrum von Polyethylen, EMAA 2.2, 1860 bar, $F_{M A A}=0.024$, EMAA 2.10, 1300 bar, $F_{M A A}=0.026$.

Abbildung 4.12 zeigt, dass es durch den Einbau von MAA in das Copolymer zu einer breiten Absorbanz der OH-Gruppen kommt (siehe auch ${ }^{[7]}$ ). Die stark ausgeprägten Signale bei 2916 und $2849 \mathrm{~cm}^{-1}$ sind $\mathrm{CH}-\mathrm{Abs}$ orbanzen zu zuordnen. Es kann somit für den $\mathrm{OH}-$ Schwingungsanteil keine aussagekräftige Integration der Signalintensitäten über den gezeigten Bereich vorgenommen werden. Bei genauerer Betrachtung kann die Bildung einer Schulter bei $2960 \mathrm{~cm}^{-1}$ für die Säurecopolymere beobachtet werden, wie in Abbildung 4.13 gezeigt. Dazu wurden die Absorbanzen im Bereich von 3000 bis $3100 \mathrm{~cm}^{-1}$ übereinander gelegt.

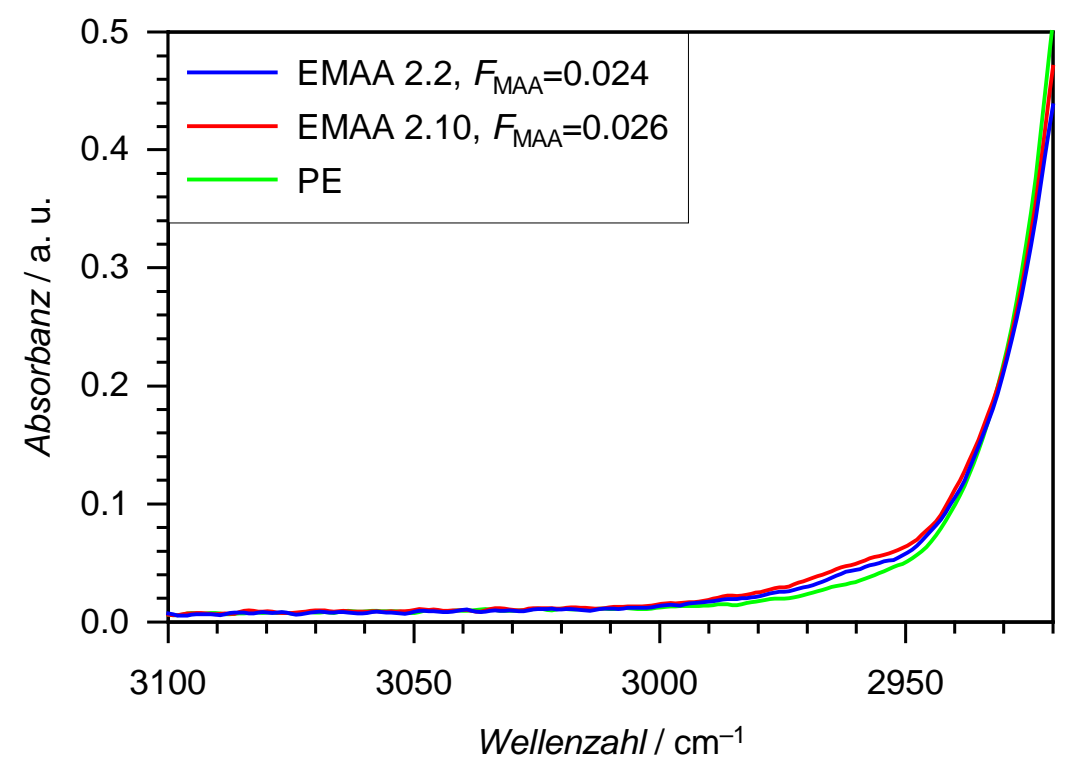

Abbildung 4.13: ATR-IR-Spektrum von Polyethylen; EMAA 2.2, $1860 \mathrm{bar}, F_{M A A}=0.024$, EMAA 2.10, 1300 bar, $F_{M A A}=0.026$, Detailansicht . 
Es bedarf der Klärung, ob diese Schulter lediglich auf den geringen MAAKonzentrationsunterschied von 0.2 mol-\% zurückzuführen ist, oder ob anhand dieses Signals eine Unterscheidungsmöglichkeit für Hoch- und Niederdruckproben gefunden wurde. Die Probenreihe EMAA 27 weist eine Hochdruckprobe mit einem höheren gemessenem MAA-Gehalt (EMAA 27.5, $\quad F_{\mathrm{MAA}}=0.048$ ) als die Niederdruckprobe (EMAA 27.8, $F_{\mathrm{MAA}}=0.042$ ) auf. Die theoretisch bestimmte Zusammensetzung weist den gleichen Trend auf (EMAA 27.5, $F_{\mathrm{MAA}}=0.049$, EMAA 27.8, $\left.F_{\mathrm{MAA}}=0.045\right)$.

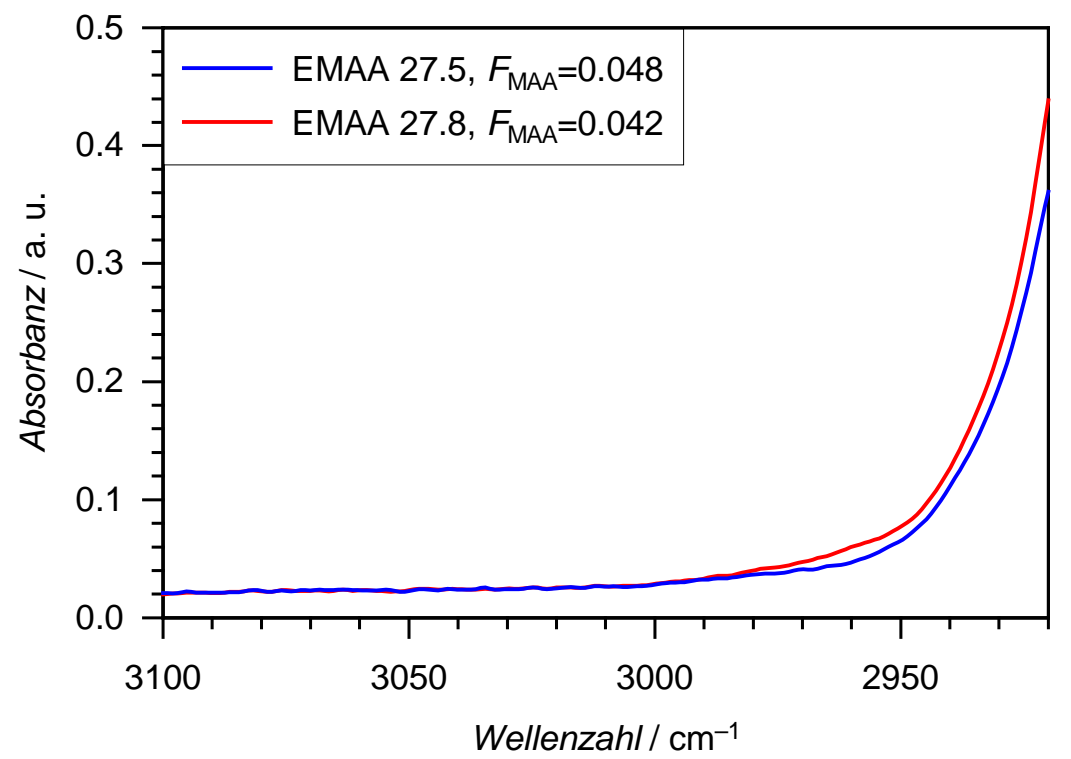

Abbildung 4.14: ATR-IR-Spektrum von EMAA 27.5, 2300 bar, $F_{M A A}=0.048$, EMAA 27.8, 1400 bar, $F_{M A A}=0.042$.

Aus Abbildung 4.14 ist ein Unterschied für eine Hoch- und Niederdruckprobe zu erkennen. Die Niederdruckprobe weist trotz geringerem MAA-Gehalt im Copolymer eine höhere Absorbanz im Bereich von $2960 \mathrm{~cm}^{-1}$ auf. Der Säuregehalt im Copolymer allein kann diesen Effekt somit nicht erklären, da sowohl Niederdruckproben mit höherem MAAGehalt im Copolymer als auch mit niedrigerem MAA-Gehalt als die entsprechende Hochdruckprobe eine höhere Absorbanz als die Hochdruckproben aufweisen. In der Literatur wird in diesem Wellenzahlenbereich mit die größte Absorbanz für Carbonsäuredimere beobachtet. ${ }^{[6]}$ Das ist ein weiteres Indiz dafür, dass die Schulter durch $\mathrm{OH}-$ Streckschwingungen von gekoppelten Säuren verursacht wird.

Mit Hilfe des Ansatzes, dass Niederdruckproben einen nicht-statistischen Aufbau im Vergleich zu den Hochdruckproben aufweisen und dem Konzept des „Chemical Confinements“, das in Kapitel 5.2.1 beschrieben ist, lässt sich die höhere Absorbanz der 
Niederdruckproben im Bereich von $2960 \mathrm{~cm}^{-1}$ erklären. Eine höhere Anzahl an WBB kann zu der beobachteten höheren Absorbanz führen.

Für alle anderen Probenreihen wurde ebenfalls eine höhere Absorbanz für die Niederdruckproben gefunden, wie Abbildung 4.15 zeigt. Zur Quantifizierung dieses Effektes wurde über die Absorbanz im Bereich von 2939 bis $3000 \mathrm{~cm}^{-1}$ integriert. Da die Lage der Absorbanz in diesem Bereich eine Abhängigkeit vom Anteil der Säure im Copolymer (breite Absorbanz der OH-Streckschwingung der Säuredimere im Bereich von 2300 bis $3400 \mathrm{~cm}^{-1}$ ) aufweist, wurde als Grundlinienpunkt für die Integration ein Basislinienpunkt bei $3000 \mathrm{~cm}^{-1}$ gewählt. Somit ergibt sich automatisch eine Normierung auf den MAA-Gehalt im Copolymer und die resultierenden integrierten Absorbanzen im Bereich von 2939 bis $3000 \mathrm{~cm}^{-1}$ können direkt in Relation gesetzt werden.

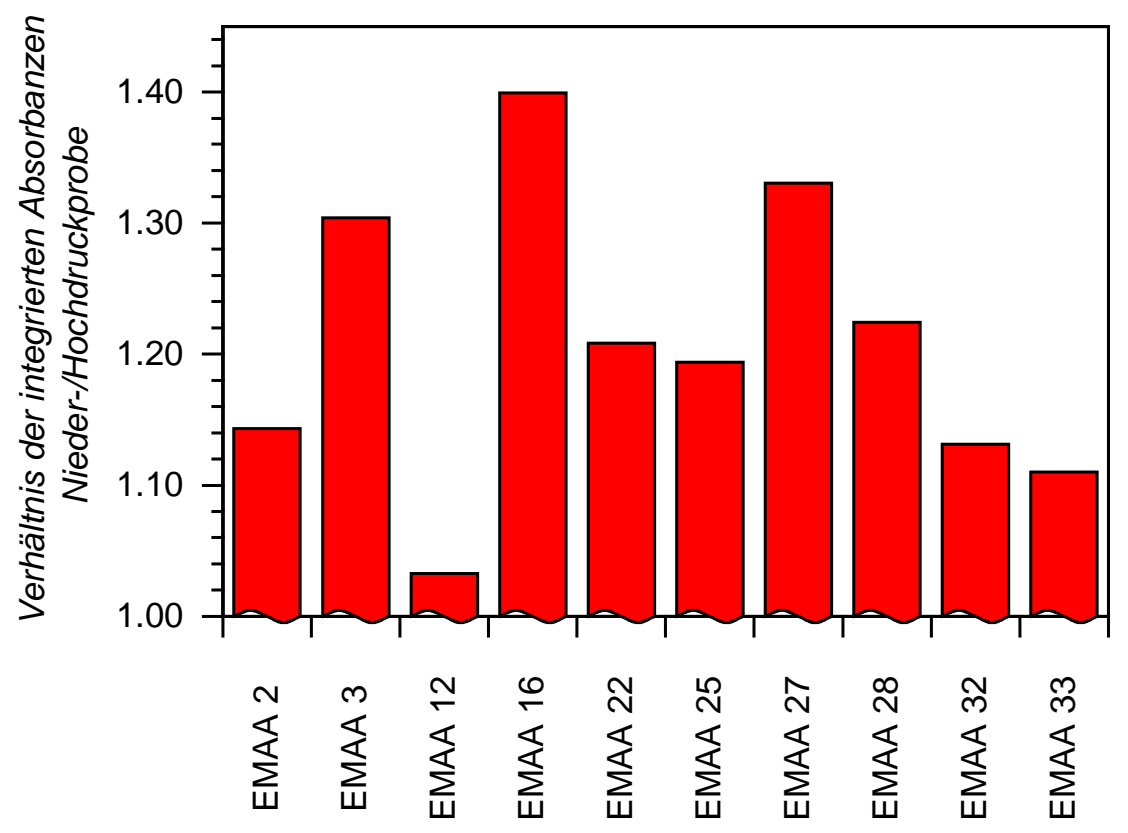

Abbildung 4.15: Quotienten der integrierten Absorbanzen von Nieder- und Hochdruckproben.

Abbildung 4.15 zeigt für alle Niederdruckproben der verwendeten Probenreihen einen höheren Wert für die integrierten Absorbanzen im Bereich von 2939 bis $3000 \mathrm{~cm}^{-1}$. Auffällig klein ist der Wert für die Probenreihe EMAA 12, wo der Gehalt an MAA im Copolymer lediglich 0.8 mol-\% beträgt. Aufgrund des geringen Säureanteils verhalten sich beide Proben nahezu ähnlich zu Polyethylen. Es ist somit recht unwahrscheinlich, dass andere Effekte, wie zum Beispiel eine unterschiedliche Dichte der Reaktionsmischung 
(siehe Kapitel 4.4), zur Erklärung der durchweg höheren integrierten Absorbanzen der Niederdruckproben im besagten Bereich führen.

\subsubsection{Auswirkung des MAA-Gehalts auf das Verhältnis der integrierten} Absorbanzen Nieder-/Hochdruckprobe

Um einen Eindruck über die Auswirkung des MAA-Gehalts im Copolymer auf die integrierten Absorbanzen zu erhalten, wurde in Abbildung 4.16 das Verhältnis der integrierten Absorbanzen Nieder-/Hochdruckprobe gegen den bestimmten Gehalt an MAA im Copolymer der Niederdruckprobe aufgetragen.

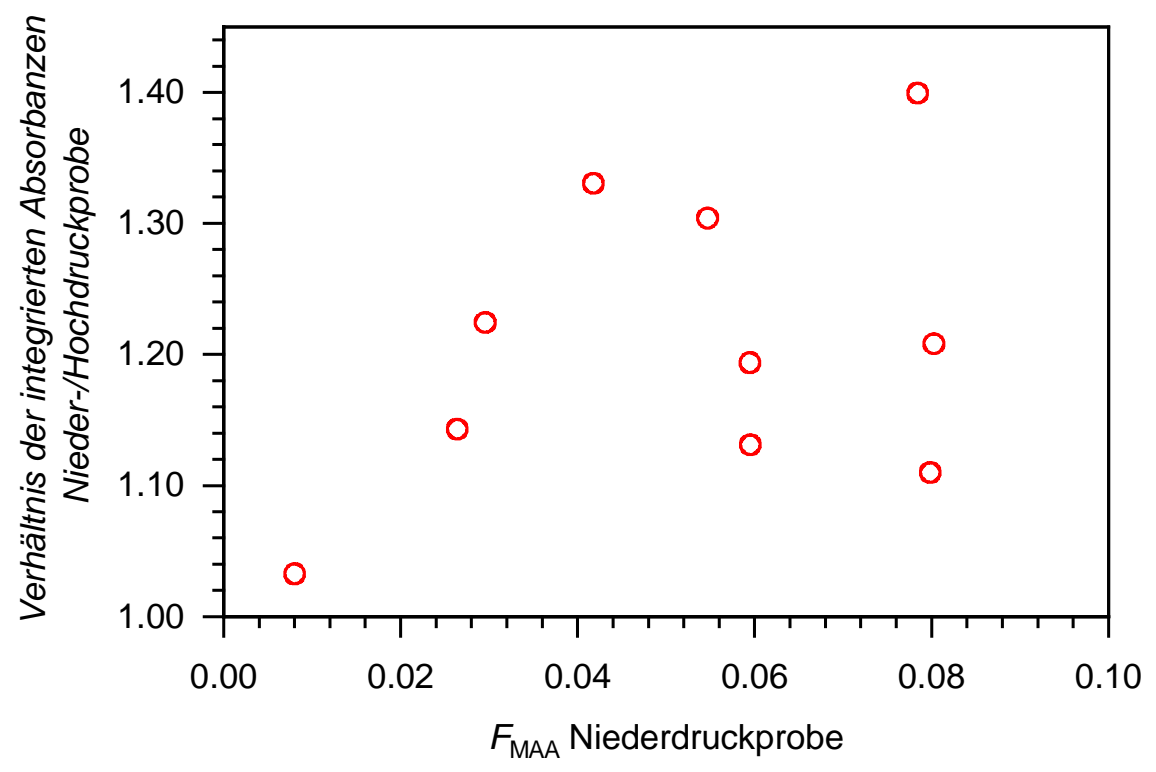

Abbildung 4.16: Quotienten der integrierten Absorbanzen von Nieder- und Hochdruckproben aufgetragen gegen den mittels ATR-FT-IRSpektroskopie bestimmten MAA-Gehalt der Niederdruckproben.

Für kleine MAA-Gehalte ist in Abbildung 4.16 mit steigendem MAA-Gehalt zunächst eine Zunahme des Integralverhältnisses zu beobachten. Somit wird für kleine MAA-Gehalte ein eher geringer Unterschied für Hoch- und Niederdruckproben beobachtet. Diese Erkenntnisse decken sich mit den in Kapitel 4.10 und 4.11 gemachten Erkenntnissen. Für höhere MAA-Gehalte tritt für verschiedene Probenreihen mit nahezu gleichem Säuregehalt eine starke Streuung der Werte auf. Für hohe Säuregehalte wird somit eine Art Sättigung beobachtet. Das bedeutet eine weitere Erhöhung des Säureanteils führt nicht mehr zur weiteren Erhöhung des Integralverhältnisses. 


\subsubsection{Auswirkung der Synthesetemperatur und des Monomerumsatzes auf das Verhältnis der integrierten Absorbanzen Nieder-/Hochdruckprobe}

Um einen eventuellen Einfluss der Synthesetemperatur und des Gesamtmonomerumsatzes auf das Verhältnis der integrierten Absorbanzen Nieder-/Hochdruckprobe festzustellen wurde analog zu Kapitel 4.3.2 verfahren und das Verhältnis der integrierten Absorbanzen Nieder-/Hochdruckprobe gegen die Synthesetemperatur (Abbildung 4.17) und gegen den Umsatz (Abbildung 4.18) aufgetragen.

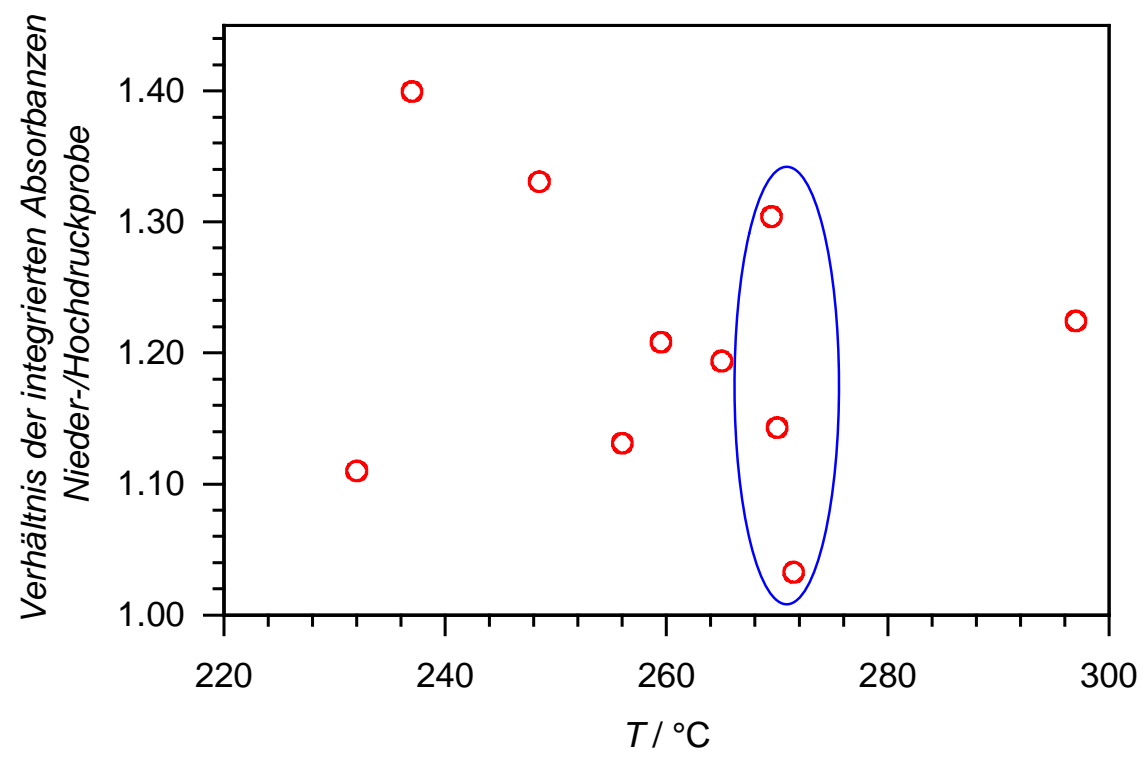

Abbildung 4.17: Quotienten der integrierten Absorbanzen von Nieder- und Hochdruckproben aufgetragen gegen die Synthesetemperatur der Niederdruckproben.

Aus Abbildung 4.17 ist zu entnehmen, dass scheinbar keine systematische Abhängigkeit des Integralverhältnisses von der Temperatur besteht. Besonders auffällig ist die breite Streuung der Werte für das Integralverhältnis bei den Probereihen EMAA 2, 3 und 12 (Kennzeichnung durch Ellipse), wo eine nahezu konstante Synthesetemperatur von 270 bis $275{ }^{\circ} \mathrm{C}$ vorliegt. Diese Ergebnisse weisen darauf hin, dass das Verhältnis von integrierten Absorbanzen für Nieder-/Hochdruckprobe nicht durch zum Beispiel Verzweigungen im Copolymer beeinflusst werden. Sollten Verzweigungen einen Einfluss haben, so wird ein Temperatureinfluss erwartet (siehe auch Kapitel 4.10.3). 


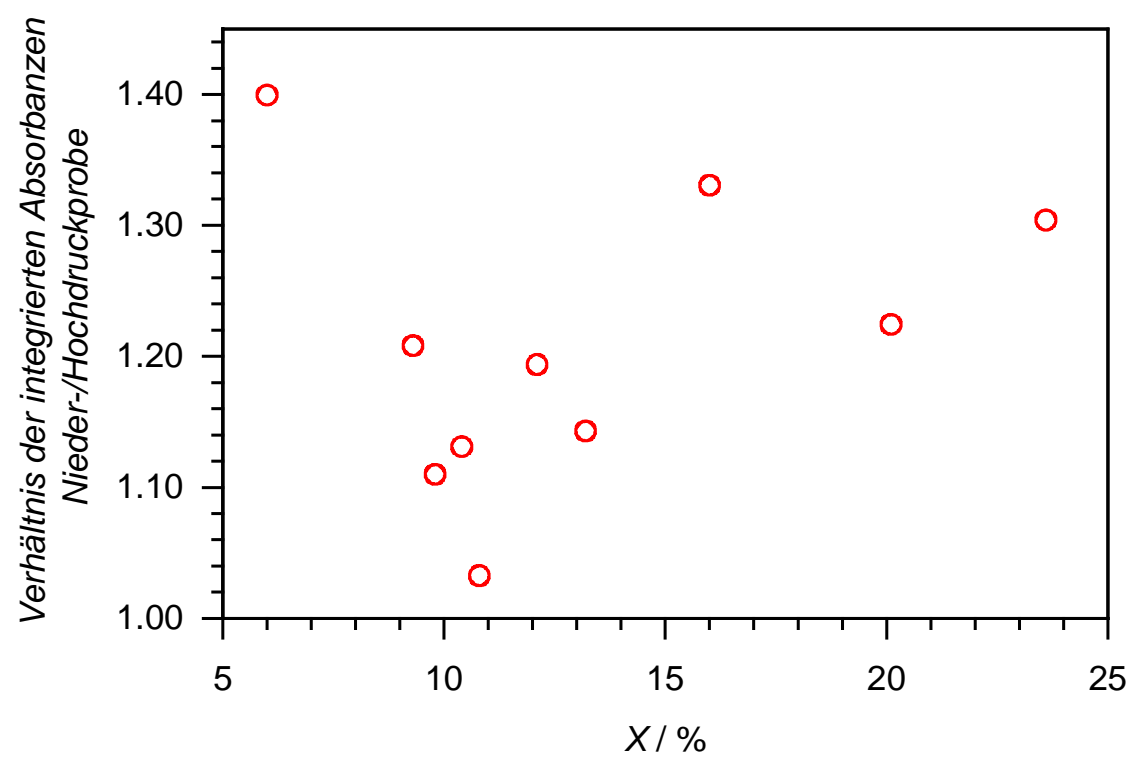

\section{Abbildung 4.18: Quotienten der integrierten Absorbanzen von Nieder- und Hochdruckproben aufgetragen gegen den Gesamtmonomerumsatz der Niederdruckproben.}

In Abbildung 4.18 wurde analog zu Abbildung 4.17 für den Umsatz verfahren. Hier wird ebenfalls keine systematische Abhängigkeit des Integralverhältnisses vom Umsatz gefunden. Bei niedrigem Umsatz liegt der Wert für das Verhältnis der integrierten Absorbanzen Nieder-/Hochdruckprobe am höchsten. Für höhere Umsätze kommt es zu einer starken Streuung der Werte. Aufgrund der Umsatzabhängigkeit der ausgebildeten Verzweigungen im Copolymer erscheinen somit Verzweigungen die beobachteten Unterschiede in den Nieder- und Hochdruckproben (siehe Kapitel 4.3.1) nicht erklären zu können.

\subsubsection{IR-spektroskopische Untersuchungen an zyklischen Anhydriden der Ethen-Methacrylsäure-Copolymere}

Ein in der Literatur ${ }^{[8]}$ beschriebenes Verfahren, das auf der Bildung von zyklischen Anhydriden von benachbarten Säuregruppen beruht, wurde ebenfalls in dieser Arbeit durchgeführt. Die in der Literatur ${ }^{[8]}$ angegebene Carbonylschwingung für die zyklischen Ahydride bei $1770 \mathrm{~cm}^{-1}$ wurde von weiteren Signalen überlagert, die höchstwahrscheinlich auf die verwendeten Lösungsmittel (Ansul-Ether und Decalin) zurückzuführen sind, so dass eine quantitative Auswertung der erhaltenen Spektren in Bezug auf den relativen Anteil an benachbarten Säuregruppen im Copolymer nicht möglich war. 


\subsection{Berechnung der Dichte der Reaktionsmischung im Reaktor}

Bei der Synthese der Copolymere werden in dieser Arbeit unterschiedliche Reaktionsdrücke benutzt. Die Hochdruckproben werden in vielen Fällen bei 2300 bar und die Niederdruckproben bei 1300 bar hergestellt. In diesem Abschnitt wird die Dichte der Reaktionsmischung unter der Annahme berechnet, dass es sich um das System Ethen/Polyethylen handelt. Der Beitrag der Methacrylsäure kann bei den typischerweise verwendeten Gehalten im Dosierstrom von $f_{\mathrm{MAA}}=0.001$ vernachlässigt werden. Die Berechnung erfolgt mittels der folgenden Gleichungen. ${ }^{[9]}$

$$
\begin{array}{ccc}
\rho=\frac{1}{\frac{1-g_{\mathrm{P}}}{\rho_{\mathrm{E}}}+\frac{g_{\mathrm{P}}}{\rho_{\mathrm{P}}}} \quad \text { wobei: } & \text { Gleichung } 4.2 \\
\rho_{\mathrm{E}}=1995.85-601.2 \cdot \lg \left(\frac{p}{1000}\right)+593.3 \cdot \lg \left(\frac{1}{T}\right)-335.8 \cdot \lg \left(\frac{p}{1000}\right) \cdot \lg \left(\frac{1}{T}\right) \quad \text { Gl. } 4.3 \\
\rho_{\mathrm{P}}=\frac{1}{\left(9.61 \cdot 10^{-4}+7.0 \cdot 10^{-7} \cdot T-5.3 \cdot 10^{-8} \cdot p\right)} \quad \text { Gleichung 4.4 }
\end{array}
$$

mit: $\quad \rho: \quad$ Dichte im Reaktor in $\mathrm{g} \cdot \mathrm{l}^{-1}$

$g_{\mathrm{P}}: \quad$ Gewichtsanteil an Polymer im Reaktor

$\rho_{\mathrm{E}}$ : Dichte Ethen unter Reaktionsbedingungen in $\mathrm{g} \cdot \mathrm{l}^{-1}$

$\rho_{\mathrm{P}}$ : $\quad$ Dichte Polymer unter Reaktionsbedingungen in $\mathrm{g} \cdot \mathrm{l}^{-1}$

\subsubsection{Vergleich der Dichte im Reaktor für Hoch- und Niederdruckproben}

Nach Gleichung 4.2 wurde die Dichte im Reaktor berechnet. Hierzu wurde exemplarische eine Temperatur von $250{ }^{\circ} \mathrm{C}$, ein Monomerumsatz von $20 \%$, ein Druck für eine Hochdruckprobe von 2300 bar und ein Druck für eine Niederdruckprobe von 1300 bar angenommen. Die resultierenden Dichten sind in Tabelle 4.3 gegeben. 


\begin{tabular}{|c|c|c|c|c|}
\hline $\begin{array}{c}\text { Temperatur } \\
/{ }^{\circ} \mathrm{C} \\
\end{array}$ & $\begin{array}{l}\text { Druck } \\
\text { / bar }\end{array}$ & $\begin{array}{c}\text { Umsatz } \\
1 \%\end{array}$ & $\begin{array}{c}\text { Dichte im Reaktor } \\
\qquad /\left.\mathrm{g} \cdot\right|^{-1}\end{array}$ & Dichte Hoch-/Niederdruck \\
\hline 250 & 2300 & 20 & 539.0 & 1.17 \\
\hline 250 & 1300 & 20 & 462.2 & \\
\hline 300 & 2300 & 20 & 519.4 & 1.18 \\
\hline 300 & 1300 & 20 & 439.2 & \\
\hline 250 & 2300 & 10 & 516.4 & 1.18 \\
\hline 250 & 1300 & 10 & 439.2 & \\
\hline
\end{tabular}

Tabelle 4.3: $\quad$ Dichte im Reaktor sowie das Verhältnis der Dichte im Reaktor Hoch- (2300 bar)/Niederdruckprobe (1300 bar) bei gegebenen Werten für Temperatur, Druck und Umsatz

Der Tabelle 4.3 kann entnommen werden, das für die Hochdruckprobe bei einer Reaktionstemperatur von $250{ }^{\circ} \mathrm{C}$ eine um $17 \%$ höhere Dichte im Vergleich zur Niederdruckprobe vorliegt. Bei einer Temperatur von $300{ }^{\circ} \mathrm{C}$ erhöht sich dieser Wert geringfügig auf 18\%. Bei Halbierung des Umsatzes von $20 \%$ auf $10 \%$ bei einer Reaktionstemperatur von $250{ }^{\circ} \mathrm{C}$ ändert sich der Wert ebenfalls von $17 \%$ auf $18 \%$.

Die Dichte im Reaktor weist somit im betrachteten Bereich von 250 bis $300{ }^{\circ} \mathrm{C}, 1300$ bis 2300 bar und Umsätze von $20 \%$ und $10 \%$ eine starke Druckabhängigkeit und nur eine sehr schwache Temperatur- und Umsatzabhängigkeit auf.

Es gilt zu untersuchen, ob der Dichteunterschied der Reaktionsmischung die in dieser Arbeit mittels verschiedener Analysen aufgezeigten Unterschiede zwischen einer Hochund einer Niederdruckprobe erklären kann. In Kapitel 4.10.1 sind DSC-Analysen beschrieben, die an den synthetisierten Copolymeren durchgeführt wurden. Ein eventueller Einfluss der Dichte der Reaktionsmischung kann anhand von Abbildung 4.27 abgeschätzt werden. Es liegt ein geringer Säureanteil im Copolymer $\left(F_{\mathrm{MAA}}=0.006\right)$ für die Probenreihe EMAA 12 vor. Es kann kein zusätzlicher Übergang für die Niederdruckprobe bei Temperaturen unterhalb des Glasübergangs bei ca. $35^{\circ} \mathrm{C}$ beobachtet werden. Es erscheint somit sehr unwahrscheinlich, dass der Dichteunterschied im Reaktor für Hoch- und Niederdruckproben Effekte, wie sie für die Niederdruckprobe EMAA 2.10 in Abbildung 4.25 in Form eines zusätzlichen Minimums im Wärmefluss gefunden wurden, verursachen kann. Die schwache Temperatur- und Umsatzabhängigkeit vermag ebenfalls nicht, die in Kapitel 4.10.3 beobachtete Temperaturabhängigkeit der Ausbildung der zusätzlichen Relaxation, gezeigt in Abbildung 4.30, zu erklären. 


\subsection{Thermogravimetrische Analyse}

Die thermogravimetrische Analyse (TGA) gibt Aufschluss über einen eventuellen Masseverlust der eingesetzten Probe während eines definiertem Aufheizvorgangs. ${ }^{[10]}$ Eventuelle Zersetzungsvorgänge können zu einer Abnahme des Probengewichtes führen. Zersetzungsprozesse, die eventuell beim notwendigem Aufschmelzen der Proben während der Probenpräparationen auftreten, können auf diese Weise beobachtet werden.

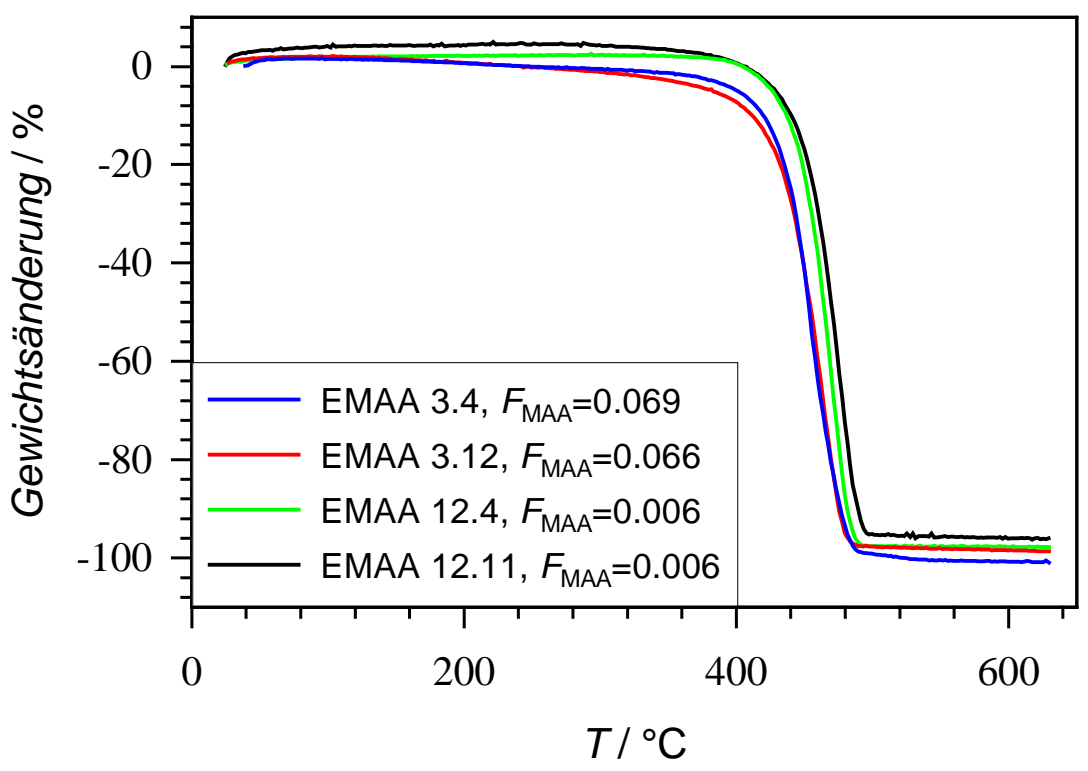

Abbildung 4.19: Thermogravimetrische Analyse von Copolymeren mit hohem (EMAA 3; EMAA 3.4, 2300 bar, EMAA 3.12, 1300 bar) und mit niedrigem (EMAA 12; EMAA 12.4, 1300 bar, EMAA 12.11, 2300 bar) MAA-Gehalt.

Keine der analysierten Proben zeigt einen erheblichen Masseverlust bei Temperaturen von unter $300{ }^{\circ} \mathrm{C}$ (Abbildung 4.19). Der Aufschmelzvorgang der Polymerproben führte bei den verwendeten Temperaturen nicht zu einem Masseverlust. Bei Temperaturen von über $500{ }^{\circ} \mathrm{C}$ ist nichts mehr von der ursprünglichen Probe vorhanden. Die Proben haben sich vollständig zersetzt und die Bestandteile sind in den gasförmigen Zustand übergegangen.

Die beiden untersuchten Probenreihen EMAA 3 und EMAA 12 unterscheiden sich signifikant im Temperaturbereich unterhalb der Zersetzung. Hier kann für die MAAreichen Proben eine kontinuierliche Massenabnahme beobachtet werden. Die Massenänderung beträgt bei der Hochdruckprobe $-2.95 \%$ und bei der Niederdruckprobe $-4.25 \%$. 
Das mehrtägige Trocknen der Copolymerproben bei einer Temperatur von ca. $70{ }^{\circ} \mathrm{C}$ unter Hochvakuum zeigte auf einer Analysenwaage mit einem Messbereich von $0.1 \mathrm{mg}$ keinen Gewichtsverlust der Proben. Restmonomer, das unter diesen Bedingungen verdampft, sollte also zu beobachten sein. Aus den erhaltenen Messdaten lässt sich für die TGA eine Massenbestimmung im Bereich von $0.01 \mathrm{mg}$ abschätzen. Die beobachte Massenabnahme könnte somit auf eine genauere Messmethode zurückzuführen sein und es könnte sich doch um Restmonomer handeln. Ein weiterer Erklärungsansatz beruht auf dem Vorhandensein von Wasserstoffbrückenbindungen (WBB). Copolymere, die einen hohen Anteil an MAAEinheiten besitzen, haben die Möglichkeit, eine höhere Anzahl an WBB auszubilden. Diese Wasserstoffbrückenbindungen können intramolekular strukturiert sein oder sich intermolekular auf eine weitere Polymerkette erstrecken. Möglich ist es aber auch, dass das polare Monomer Methacrylsäure eine WBB zu einer Polymerkette eingeht. Diese Monomereinheit ist nicht ohne weiteres im Hochvakuum zu entfernen. Ein Auftrennen der WBB ist möglicherweise erst bei höheren Temperaturen gegeben. Der höhere Massenverlust der Niederdruckprobe ist eventuell durch die spezielle Anordnung der Methacrylsäuregruppen zu erklären. Bei einer örtlich hohen Konzentration von MAAEinheiten ist es prinzipiell leichter eine monomere Säureeinheit anzulagern. Eine polare Umgebung stabilisiert die MAA-Monomere, wohingegen eine unpolare Umgebung die Monomere eher abstößt. 


\subsection{Bestimmung der Mikrohärte}

Wie auch in Kapitel 4.10.3 erwähnt, weisen die Hoch- und Niederdruckproben unterschiedliche Härten auf. Eine Härtemessung an den Copolymerproben, die auf dem Prinzip des Eindringens eines Probenkörpers ${ }^{[11]}$ beruht, sollte die in Kapitel 4.10.3 vorgestellten Tendenzen zeigen. Die Analysen wurden für die Probenreihen durchgeführt, bei denen eine Variation des MAA-Gehalts vorgenommen wurde.

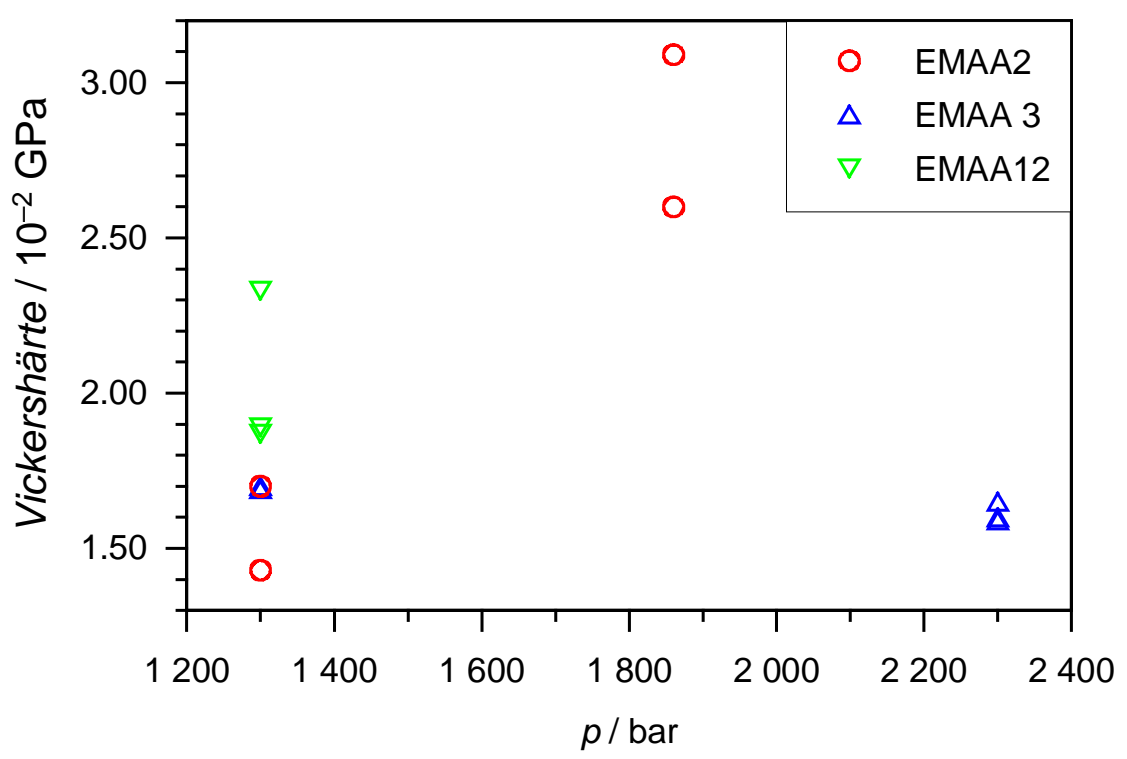

Abbildung 4.20: Härtebestimmung nach Vickers ${ }^{[11]}$ für unter verschiedenen Bedingungen synthetisierte EMAA-Copolymere: EMAA 12 (grün): niedriger Säuregehalt, EMAA 2 (rot): mittlerer Säuregehalt, EMAA 3 (blau): hoher Säuregehalt (siehe auch Tabelle 4.2).

Nur für die Probenreihe EMAA 2 und EMAA 3 ließen sich sowohl Hoch- als auch Niederdruckprobe vermessen, was Abbildung 4.20 zeigt. Bei der Probenreihe EMAA 12 konnte nur die Niederdruckprobe gemessen werden, da ein homogenes Aufschmelzen der Hochdruckprobe nicht erfolgen konnte. Die verschiedenen Messwerte für einen gegebenen Synthesedruck ergeben sich durch mehrmaliges Messen ein und derselben Probe an verschiedenen Stellen. So entsteht ein Eindruck über den möglichen Fehlerbereich einer Messung. Prinzipiell ist festzuhalten, dass dieses Verfahren der Härtemessung eher bei Metallen verwendet wird und die hier verwendeten Polymere für diese Analyse eigentlich zu weich sind. Die Messungen an den Probenreihen EMAA 2 bestätigen den Trend, der mittels der DMA-Messungen gefunden wurde. Die Hochdruckprobe (EMAA 2.2) weist eine nahezu doppelt so hohe Härte auf, wie die Niederdruckprobe (EMAA 2.10). Die 
Probenreihe EMAA 3 zeigt keinen signifikanten Unterschied für Hoch- und Niederdruckprobe. Unter Berücksichtigung eines Fehlers von $25 \%$ des gemessenen Wertes kann die Hochdruckprobe (EMAA 3.4) allerdings einen deutlich höheren Wert zeigen, als die Niederdruckprobe (EMAA 3.12). Die Hochdruckprobe EMAA 3.4 liegt deutlich unterhalb des für die Hochdruckprobe EMAA 2.2 bestimmten Wertes. Von den Niederdruckproben ist die Probe EMAA 12.11 die härteste. Messungen an einem PE-Homopolymer führten zu Werten für die Vickershärte von ca. $10 \cdot 10^{-2} \mathrm{GPa}$. Somit ist das Homopolymer erheblich härter als die gemessenen Copolymere. Somit kann hier der Trend, dass der verstärkte Einbau von Säuregruppen in das Copolymer zu einer Erweichung des Materials führt, bestätigt werden. 


\subsection{Reissdehnung}

Die unterschiedliche Härte der Polymere, die in Kapitel 4.6 beobachtet wurde, sollte auch eine Auswirkung auf weitere mechanische Eigenschaften haben. Es wird zum Zerreissen einer härteren Probe eine höhere Reissspannung benötigt. ${ }^{[12]}$ Dadurch sollten Hochdruckproben eine höhere Reißspannung aufweisen als Niederdruckproben. Die Untersuchungen wurden an Polymerstäbchen durchgeführt, die durch das in Kapitel 3.2.12 vorgestellte Verfahren erhalten wurden.

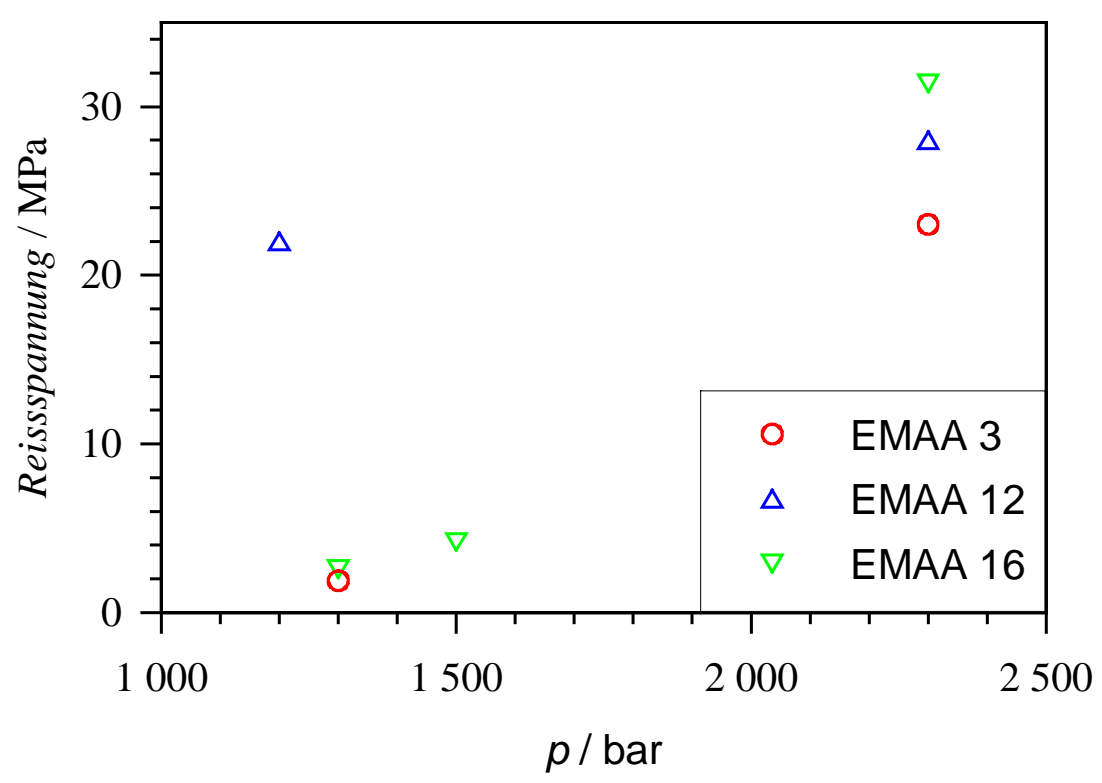

Abbildung 4.21: Verwendete Reissspannung, die zum Zerreissen der entsprechenden Polymerproben führt. Untersucht wurden die Probenreihen EMAA 3 (mittlerer Säuregehalt), EMAA 12 (niedriger Säuregehalt) und EMAA 16 (Synthese unter adiabatischen Bedingungen).

Aus Abbildung 4.21 ist ersichtlich, dass die Hochdruckproben eine höhere Reissspannung aufweisen als die dazugehörigen Niederdruckproben. Die unter adiabatischen Bedingungen hergestellten Proben verhalten sich analog zu den vorangegangenen Probenreihen. Hier wird der gezeigte Trend durch eine weitere Probe mit einem Synthesedruck von 1500 bar bestätigt. Die Niederdruckproben mit einem relativ hohem Gehalt an Säureeinheiten im Copolymer (EMAA 3 und EMAA 16, siehe Tabelle 4.1) weisen einen erheblich geringeren Wert für die Reissspannung auf, als die Niederdruckprobe mit einem geringen MAAGehalt (EMAA 12). Ein hoher Anteil von MAA-Einheiten führt bei den Niederdruckproben $\mathrm{zu}$ einer erheblich geringeren Härte und somit zu einer geringeren mechanischen Festigkeit. Bei Hochdruckproben kann diese Tendenz nicht belegt werden. 
Unter Berücksichtigung des ermittelten Fehlers von $\pm 4.2 \mathrm{MPa}$ für die Hochdruckprobe von EMAA 16 kann der entsprechende Messwert unterhalb des ermittelten Wertes für EMAA 12 rutschen. Bei den Niederdruckproben beträgt der maximale Fehler nur $\pm 0.4 \mathrm{MPa}$ (EMAA 12). Hier hebt sich also die Niederdruckprobe EMAA 12 deutlich von den beiden anderen Niederdruckproben $a b$.

Es wurde für die Niederdruckproben eine geringere Reißfestigkeit gefunden wie für die Hochdruckproben. Diese Ergebnisse stehen in guter Übereinstimmung mit den in Kapitel 4.9 erzielten Resultaten. Die Niederdruckproben mit einem hohen Methacrylsäuregehalt im Copolymer weisen eine geringere mechanische Belastbarkeit auf, als Proben mit niedrigem Säuregehalt, wie ebenfalls in Kapitel 4.9 bereits aufgezeigt. Diese beiden unterschiedlichen Verfahren führen unabhängig voneinander im Rahmen ihrer Möglichkeiten zu den gleichen Resultaten. 


\subsection{Optische Messungen an Copolymeren}

Um einen Eindruck bezüglich der optischen Eigenschaften der hergestellten Copolymer zu erhalten, wurden Messungen der Transmission, der Trübung und der Klarheit vorgenommen. Die Ergebnisse der Trübungs- und Klarheitsmessung sind ausschließlich durch die Oberfläche der zu untersuchenden Probe bestimmt. Es werden somit sehr hohe Anforderungen an die präparierte Oberfläche gestellt. Blasenbildung während der Probenpräparation muss verhindert werden. Für viele Polymere eignet sich das Verfahren des Folienblasens besonders gut um eine homogene Folie herzustellen. Dieses Präparationsverfahren stand nicht zur Verfügung und die Proben mussten unter erhöhter Temperatur in eine Tablettenform gepresst werden (siehe Kapitel 3.2.12). Da die Beschaffenheit der Probenkörper nicht optimal war, müssen die abgebildeten Werte für die Messungen eher als Richtwert betrachtet werden und sind stark fehlerbelastet. Auch für weitere Probenreihen wurden Probekörper gepresst, aber ihre Beschaffenheit machte eine Messung unpraktikabel. Ergebnisse für die Probenreihen EMAA 3 und EMAA 16 sind in Abbildung 4.22 gezeigt.

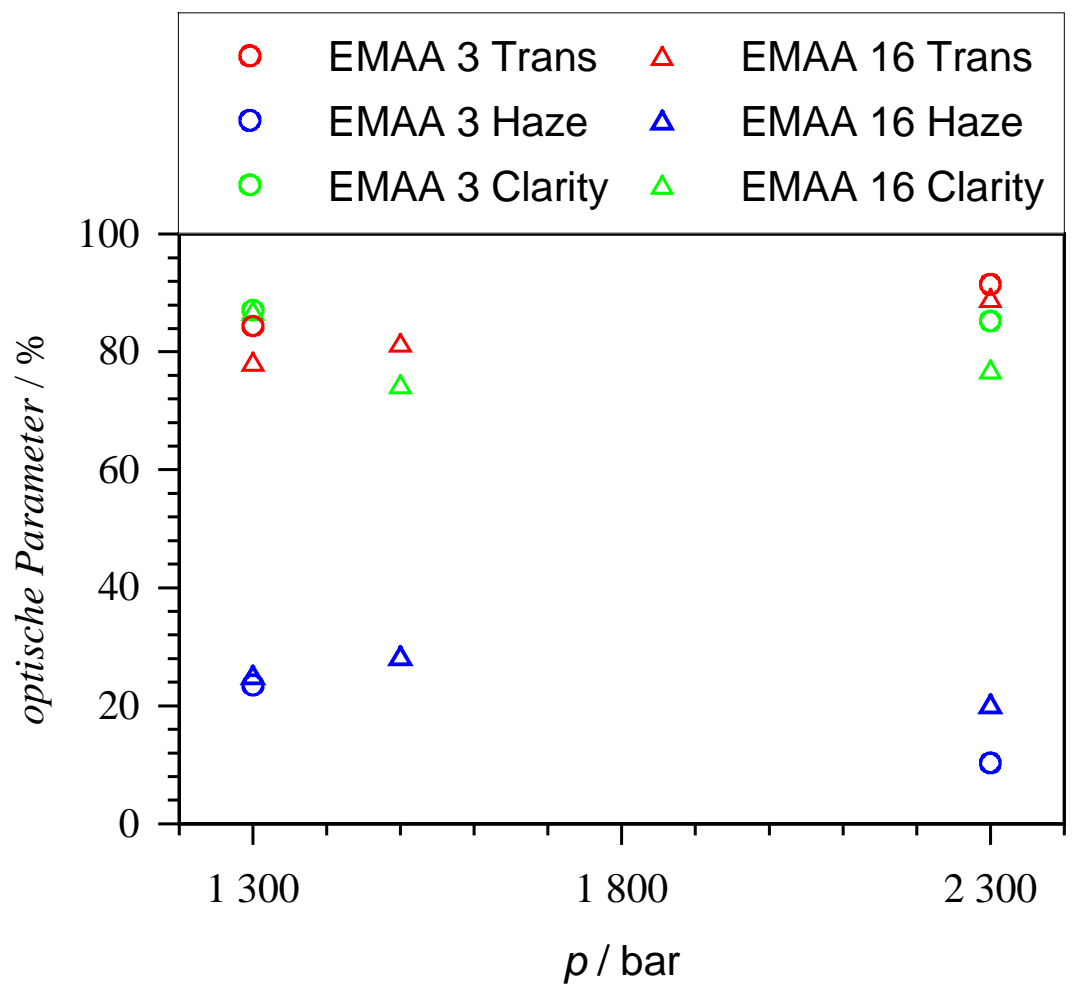

Abbildung 4.22: Optische Untersuchungen an Copolymeren mit einem hohen MAA-Anteil (EMAA 3, Kreissymbole) und unter adiabatischen Bedingungen synthetisierten Proben (EMAA 16, Dreiecksymbole). Trans bezeichnet dabei die Transmision (rot), Haze die Trübung (blau) und Clarity die Klarheit (grün). Die entsprechenden Definitionen befinden sich in Kapitel 3.2.12. 
Aufgrund der bereits beschriebenen Probleme lassen sich nicht allzu viele Erkenntnisse aus den Messungen gewinnen. Setzt man die Trübung in Relation zur Klarheit, so wird deutlich, dass die Trübung bei der Probenreihe EMAA 16 bei Synthesedrücken von 1300 bar im Vergleich zu 1500 bar zunimmt. Die Klarheit nimmt für diesen Fall ab. Beim Vergleich der beiden Hochdruckproben zeigt EMAA 16 eine höhere Trübung und eine geringere Klarheit als die Probe EMAA 3. Tendenziell führt eine geringe Trübung zu einer höheren Klarheit.

Die Konstruktion von Ausgleichsgeraden für die einzelnen Parameter würde in den Fällen der Transmission und der Trübung zumindest einen Trend aufzeigen. Die Transmission steigt mit wachsendem Synthesedruck an, die Trübung wird geringer. Für die Klarheit ist eine Entwicklungsabschätzung aus den gemessenen Daten nicht möglich. Aufgrund des beschrieben Verhältnisses der Klarheit zur Trübung einer Probe sollte die Klarheit mit steigendem Synthesedruck im Gegensatz zur Trübung ansteigen. 


\subsection{Relaxationen im Polymer}

Die Copolymere, die in dieser Arbeit synthetisiert wurden, bestehen aus unpolaren (Ethen) und polaren (Methacrylsäure) Monomereinheiten. Es können verschiedene Arten von Wechselwirkungen, unterschiedliche Beweglichkeiten der Kettensegmente und verschiedene Möglichkeiten für Vernetzungen inter- und intramolekularer Art, gegeben sein, je nach chemischer Zusammensetzung des Copolymers. Diese Vielfältigkeit der Mikrostruktur kann zu einer Vielzahl an makroskopischen Relaxationen führen, da prinzipiell jede Struktureinheit zu einer spezifischen Relaxation führen kann. Dennoch können einige charakteristische Relaxationen für Polymere beschrieben werden. Eine ausführliche Beschreibung findet sich in ${ }^{[1]}$, deswegen wird im Folgenden nur kurz auf die Relaxationen eingegangen.

\subsubsection{Kurbelwellenbewegungen}

Bei vergleichsweise tiefen Temperaturen kann für Polymere eine Kurbelwellenbewegung beobachtet werden, die von Boyer und Schatzki beschrieben wird. ${ }^{[13,14]}$ Boyer geht dabei von 6 Methylengruppen eines Polyethylenmakromoleküls aus. Abbildung 4.23 zeigt, dass die Bindungen 1 und 5 auf einer Drehachse liegen und die Bindungen 2, 3 und 4 um diese Achse rotieren können. Schatzki beschreibt diese Bewegung über 7 Bindungen, wobei Bindung 1 und 7 auf der Drehachse liegen. Nun kann eine Kurbelwellenbewegung der Bindungen 2 bis 6 stattfinden. Die resultierende Relaxationsmode ist bei einer verwendeten Frequenz von $1 \mathrm{~Hz}$ bei ca. $-120^{\circ} \mathrm{C}$ in der DMA zu beobachten. ${ }^{[14]}$

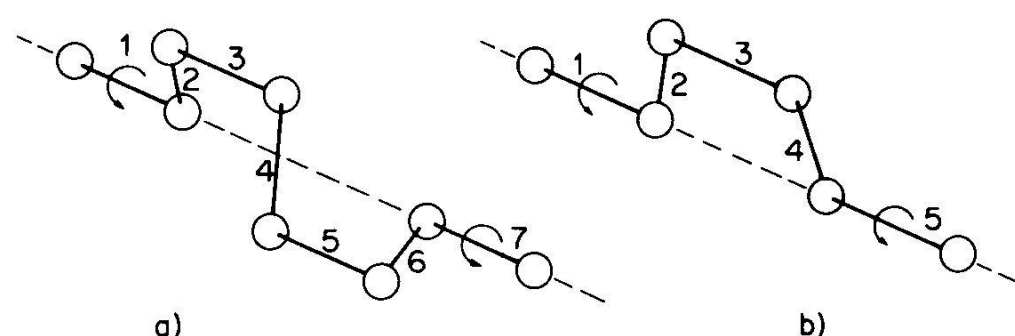

Abbildung 4.23: Schematische Darstellung der Kurbelwellenbewegung nach Schatzki (a)) und Boyer $(b)) .{ }^{[1]}$ 


\subsubsection{Segmentbewegungen}

Ein lineares Polymer ist entropisch ungünstiger als ein geknäulter Zustand, für den es mehr

Realisierungsmöglichkeiten gibt. Das Modell für Segmentbewegungen nach Rouse $^{[15,16,17]}$ für geknäulte Polymere in der Schmelze beschreibt die wesentlichen Prozesse des Glasübergangs ( $\alpha$-Relaxation). Die Kernaussage dieses Modells ist die Bewegung von ganzen Kettensegmenten. Liegt die Segmentgröße unterhalb von 100 Monomereinheiten, so wird ein Einfluss der Segmentgröße auf die Lage der Relaxation beobachtet. ${ }^{[13]}$ Kürzere Segmente benötigen eine geringere Anregungsenergie. Die für Niederdruckproben beobachte zusätzliche $\beta$-Relaxation ist in dem Bereich der Segmentbewegung anzuordnen (siehe Kapitel 5.1 und Abbildung 5.3).

\subsubsection{Abgleiten von ganzen Ketten bei Temperaturen oberhalb des Glasübergangs}

Beim Überschreiten der Glastemperatur kommt es zu einer Erweichung des Polymers bevor bei höheren Temperaturen der Übergang in das viskose Fließen einsetzt $(\alpha 2$ Übergang). In diesem Temperaturbereich kommt es zur Ausbildung von Verhakungspunkten, die ein vollständiges Abgleiten der Polymerketten verhindern. ${ }^{[13]}$ Diese Verhakungspunkte weisen Abstände von 3 bis $10 \mathrm{~nm}$ auf. ${ }^{[17]}$ Es können also Rohrsysteme entstehen, in deren Innerem eine Polymerkettenbewegung möglich ist. Diese Relaxation wird als Reptation bezeichnet. ${ }^{[13,17]}$ Sie ist für die hier untersuchten Polymersysteme nicht zu beobachten, da der Probenkörper beim Überschreiten der Glastemperatur bereits so stark verformbar ist, dass er in der DMA-Apparatur zerdrückt wird. 


\subsection{Kalorimetrische Untersuchungen mittels DSC}

Mit Hilfe der differentiellen Kalorimetrie können Phasenübergänge und Strukturumwandlungen in Materialen beobachtet werden (siehe auch Kapitel 3.2.1). Es ist keine besonders aufwändige Probenpräparation notwendig. Die Messzeit richtet sich nach dem programmierten Heizverlauf. Für die Copolymerproben kann eine Analyse bei einer konstanten Heizrate von $5{ }^{\circ} \mathrm{C} \cdot \mathrm{min}^{-1}$ im Temperaturbereich oberhalb von $-45^{\circ} \mathrm{C}$ durchgeführt werden. Somit stellt die DSC-Analyse eine schnelle und aussagekräftige Methode dar, um Strukturumwandlungen im Copolymer zu erfassen.

Becker zog zum Bespiel die mittels DSC bestimmten Schmelztemperaturen zur Bestimmung des Kurzkettenverzweigungsgrades für Polyethylen heran. ${ }^{[19]}$

Für die hier untersuchten EMAA-Copolymere wird für Niederdruckproben aufgrund ihres nicht-statistischen Aufbaus eine zusätzliche Umwandlung im Material erwartet. Diese Umwandlung sollte im Bereich der Segmentbewegung $\mathrm{zu}$ beobachten sein (siehe Kapitel 4.10.1.2). Da es zu einer chemischen Behinderung kommt (siehe Kapitel 5) wird der resultierende Phasenübergang bei tieferen Temperaturen als der eigentliche Glasübergang des Copolymeren erwartet.

Um eine direkte Vergleichbarkeit der erhaltenen Thermogramme zu gewährleisten, werden die Proben über ihren Schmelzbereich (genauer: Übergang in den Liquiduszustand, in der Chemie oft als Schmelzpunkt oder Schmelzbereich bezeichnet) hinaus erhitzt und eine Gerade an den resultierenden Kurvenverlauf oberhalb des Schmelzbereiches angepasst. Die erhaltene Funktion dient als Basislinie und wird von den Rohdaten subtrahiert. Die sich ergebende Differenz wird auf die jeweils verwendete Probenmasse normiert.

\subsubsection{DSC-Analysen an den Homopolymersystemen PE und PMAA und an Copolymerproben EMAA mit verschiedenem Methacrylsäuregehalt}

Um die Signale im Wärmefluss der Copolymerproben interpretieren zu können ist es sinnvoll, auch die Homopolymere der beiden verwendeten Monomersorten zu untersuchen. Das Thermogramm für PE und PMAA ist in Abbildung 4.24 gegeben. Für PE kann ein klarer Schmelzpeak beobachtet werden, dessen Minimum bei $120{ }^{\circ} \mathrm{C}$ liegt. Unterhalb des Schmelzübergangs, also bei tieferen Temperaturen, ist kein weiterer Übergang zu beobachten. Der Glasübergang für PE liegt je nach Kettenlänge und Verzweigungsgrad der 
Polymere weit unterhalb der hier zu realisierenden Temperatur von $-45{ }^{\circ} \mathrm{C}$ und kann somit nicht beobachtet werden.

Für das Säurehomopolymer PMAA ist der Kurvenverlauf nicht so eindeutig. Mittels Tangentenkonstruktion lässt sich bei dem gezeigten, breiten Signal ein Onset bei $46{ }^{\circ} \mathrm{C}$ bestimmen. Das Minimum liegt bei $112^{\circ} \mathrm{C}$. Hierbei kommt es wahrscheinlich zu einer Erweichung des Materials. Ein klares Signal für den Schmelzvorgang, wie bei PE beobachtet, konnte nicht gefunden werden.

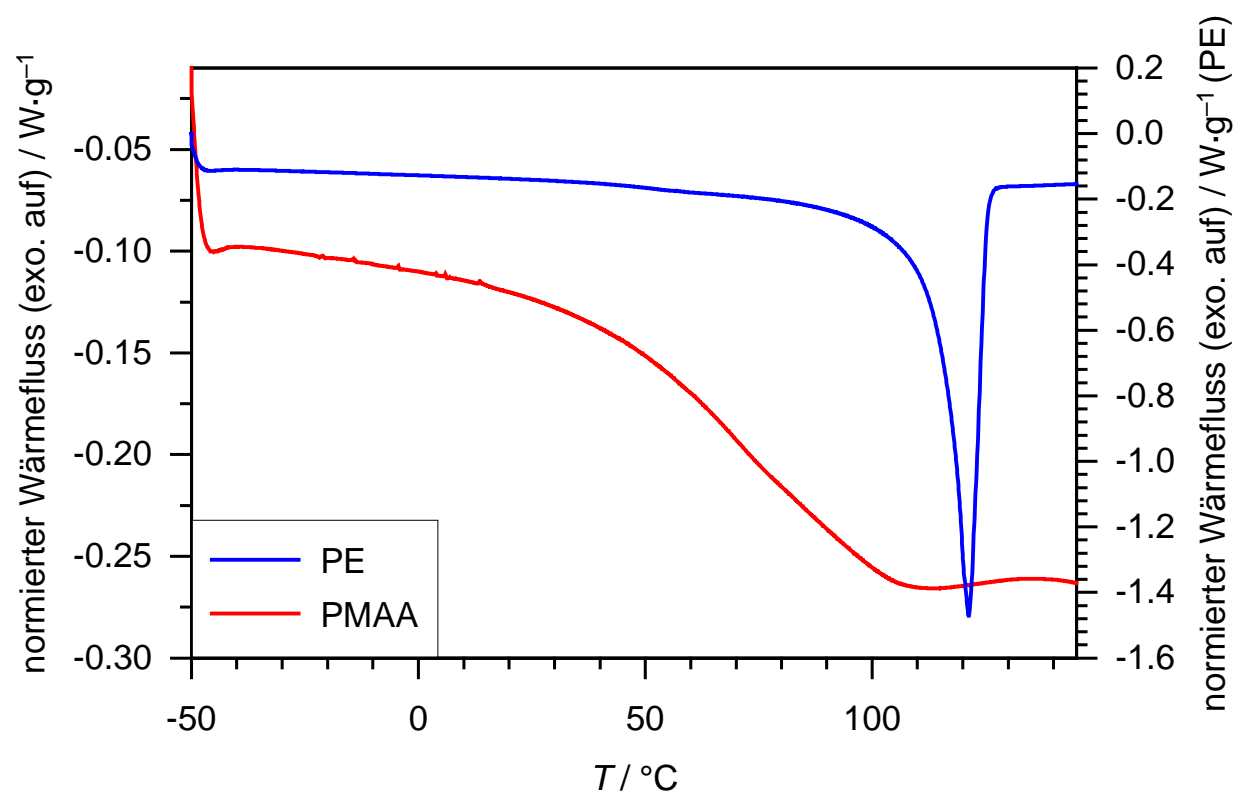

Abbildung 4.24: DSC-Thermogramm für die Homopolymere PE und PMAA bei einer Heizrate von $5^{\circ} \mathrm{C} \cdot \mathrm{min}^{-1}$. [1]

Die Thermogramme der synthetisierten Copolymerproben EMAA sind in Abbildung 4.25 bis Abbildung 4.29 gegeben. Von besonderem Interesse sind Unterschiede zwischen Hoch(blau dargestellt) und Niederdruckproben (rot und gegebenenfalls grün dargestellt) im erhaltenen Wärmeverlauf. Abbildung 4.30 zeigt nur Niederdruckproben. 


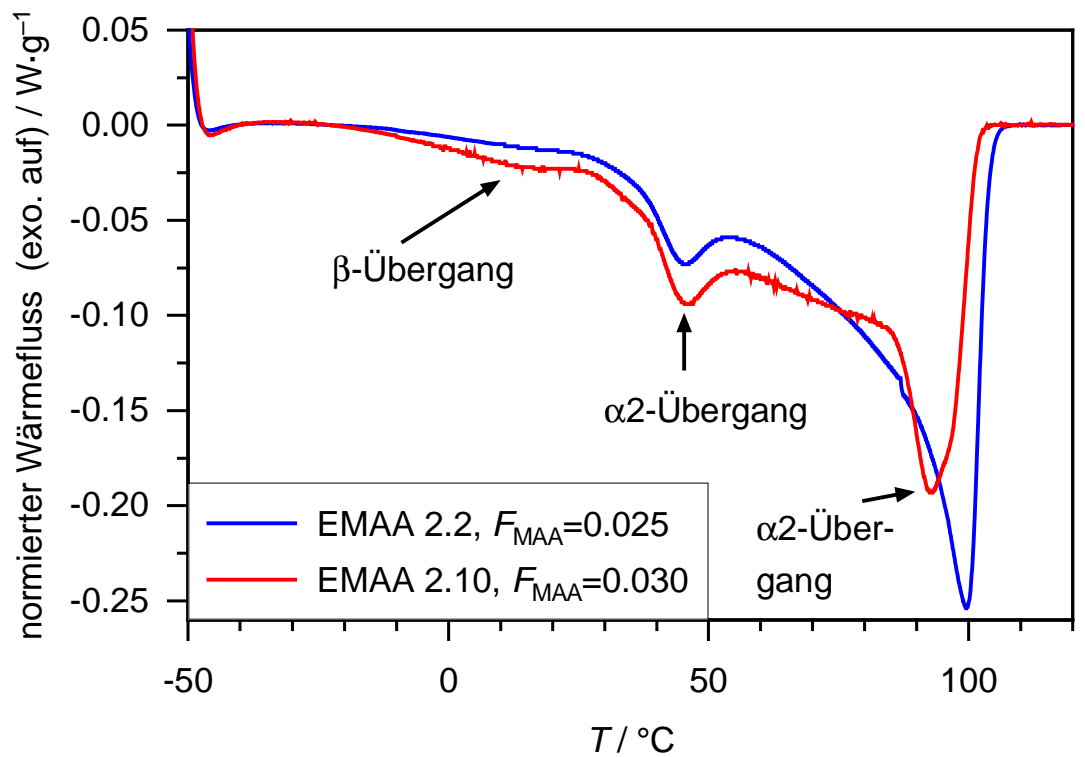

Abbildung 4.25: DSC-Thermogramm für EMAA 2.2, 1860 bar, $F_{M A A}=0.025, E M A A ~ 2.10$, 1300 bar, $F_{M A A}=0.030 .{ }^{[1]}$

Aus Abbildung 4.25 lässt sich bereits ein Unterschied für die bei unterschiedlichen Drücken hergestellten Proben feststellen. Die Hochdruckprobe (blaue Kurve), weist im Bereich von 90 bis $100{ }^{\circ} \mathrm{C}$ ein Signal für den Schmelzübergang (Übergang in das viskose Fließen) auf. Das entsprechende Minimum des Thermogramms wird als $T_{\alpha 2}$ bezeichnet. Ein zusätzlicher Übergang im Material beginnt bereits bei ca. $35{ }^{\circ} \mathrm{C}$ und ist bei ca. $53{ }^{\circ} \mathrm{C}$ vollständig beendet. Dieser Übergang entspricht dem Glasübergang des Copolymers und wird als $T_{\alpha}$ bezeichnet. Die dazugehörige Temperatur ist die Glastemperatur $T_{\mathrm{G}}$. Der Einbau von Methacrylsäure in das Polymer führt zu einer deutlichen Erhöhung der Glastemperatur im Copolymer im Vergleich zum Homopolymer PE, bei dem kein Glasübergang im gezeigten Temperaturbereich gefunden werden kann. Dadurch ist es möglich, den Glasübergang bei allen hier untersuchten Copolymerproben zu beobachten. Die Niederdruckprobe (rote Kurve) zeigt ebenfalls diese beiden Übergänge bei nahezu identischen Temperaturen wie die Hochdruckprobe. Darüber hinaus zeigt sich aber ein zusätzlicher Übergang bei tieferen Temperaturen. Da es sich hier um einen weiteren Glasübergang handelt, wird die dazugehörige Temperatur als $T_{\mathrm{G} 2}$ und das entsprechende Minimum als $\beta$ bezeichnet. 


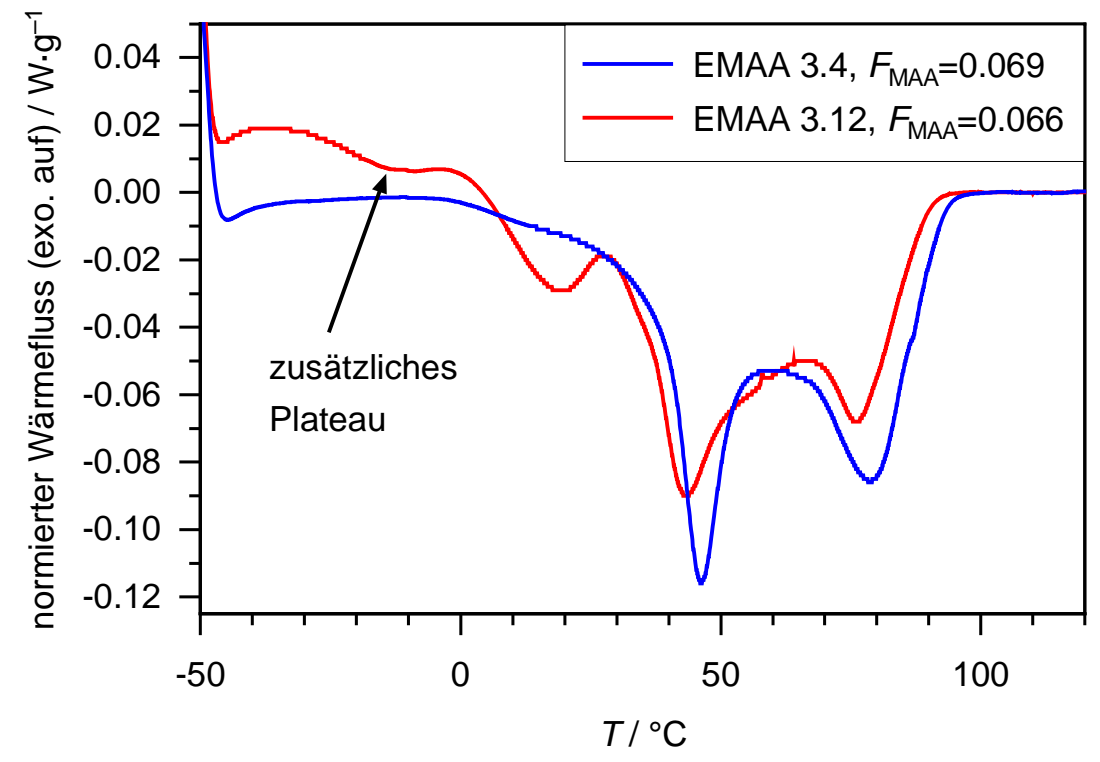

Abbildung 4.26: DSC-Thermogramm für EMAA 3.4, 2300 bar, $F_{M A A}=0.069$, EMAA 3.12, 1300 bar, $F_{M A A}=0.066 .^{[1]}$

Um diesen Übergang noch besser herauszuarbeiten, wurde für die nächste Probenreihe, bei nahezu gleicher Synthesetemperatur von ca. $270{ }^{\circ} \mathrm{C}$, der Dosierstrom an Säure erhöht, was $\mathrm{zu}$ deutlich höheren MAA-Gehalten im Copolymer von über 6 mol-\% führte. Abbildung 4.26 zeigt die Resultate der Probenreihe EMAA 3. Hier ist der zusätzliche Übergang bei tieferen Temperaturen als $T_{\mathrm{G}}$, also das $\beta$-Minimum, noch intensiver, und es kommt zu einer Aufspaltung des zusätzlichen Übergangs. Es bildet sich ein zusätzliches Plateau. Die mögliche Erklärung für die Ursache der Aufspaltung wird in Kapitel 5.2.1 gegeben. Im Zuge der Säureflussvariation wurde anschließend ein sehr geringer Säurefluss gewählt. Das führte zu Copolymeren mit einem Säureanteil von $F_{\mathrm{MAA}}=0.006$. Die Niederdruckprobe zeigt kein zusätzliches Maximum und ist dem Verlauf der Hochdruckprobe sehr ähnlich, wie Abbildung 4.27 zeigt. Der sehr geringe Säuregehalt hat offensichtlich keinen ausreichend signifikanten Einfluss auf zusätzliche Relaxationen im Copolymer. Diese Copolymerproben können aufgrund ihres geringen Säureanteils im Copolymer mit dem Homopolymer PE in Verbindung gebracht werden. Es erscheint äußerst unwahrscheinlich, dass PE, welches nahe der Phasengrenze synthetisiert wird, einen zusätzlichen Übergang aufweist. Aus diesem Grund wurde von der Synthese der entsprechenden Polyethylenhomopolymere abgesehen. 


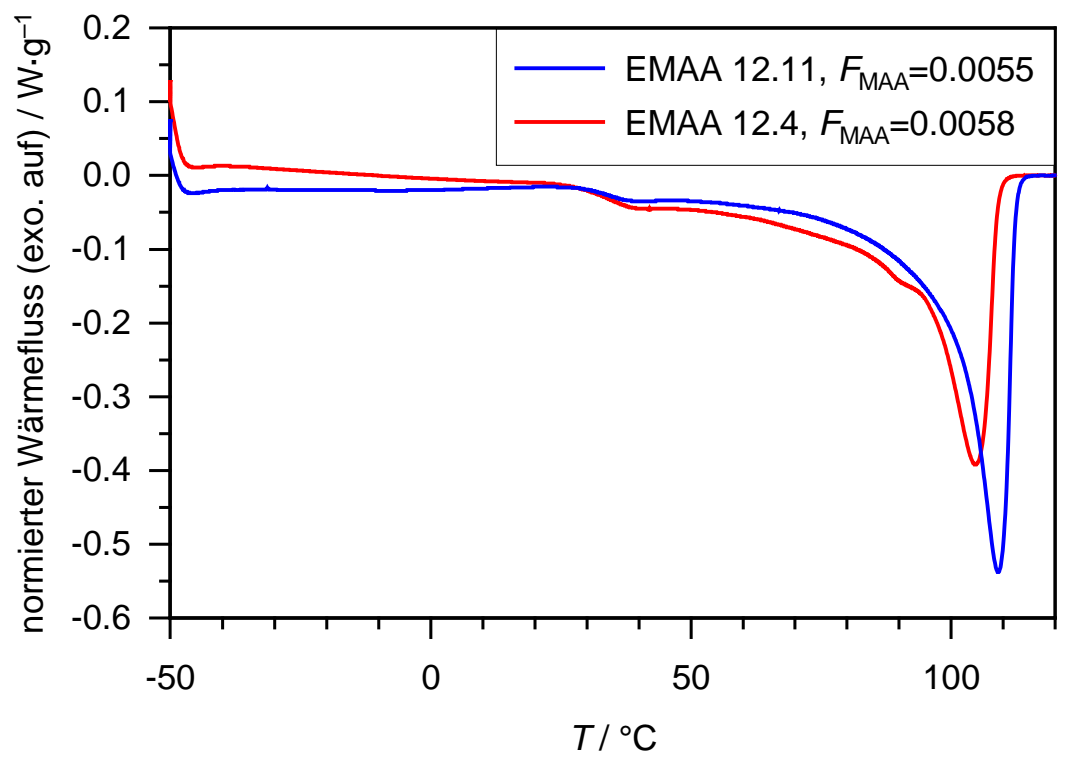

Abbildung 4.27: DSC-Thermogramm für EMAA 12.11, 2300 bar, $F_{M A A}=0.0055$, EMAA 12.4, 1300 bar, $F_{M A A}=0.0058 .{ }^{[1]}$

Aus den vorliegenden Ergebnissen kann geschlossen werden, dass eine Erhöhung des Säureanteils im Copolymer, $F_{\mathrm{MAA}}$, zu einer intensiveren Ausprägung eines $\beta$-Minimums führt. Eine Umsatzabhängigkeit dieses zusätzlichen Übergangs kann aufgrund der nachfolgenden Untersuchungen an weiteren Polymerproben ebenfalls ausgeschlossen werden.

\subsubsection{Unter adiabatischen Bedingungen hergestellte Copolymere}

Nach den Probenreihen, die sich durch eine Variation des MAA-Gehalts im Dosierstrom unterscheiden und eine erhebliche Differenz zwischen Mantel- und Reaktionstemperatur aufweisen, wurden Copolymere unter adiabatischen Bedingungen hergestellt, wobei der MAA-Anteil im Dosierstrom konstant gehalten wurde.

Unter adiabatischen Bedingungen ist in diesem Zusammenhang $\mathrm{zu}$ verstehen, dass eine Übereinstimmung der Manteltemperatur mit der direkt im Reaktionsraum gemessenen Innentemperatur vorliegt. Aufgrund der Reaktorkonstruktion wird sehr viel Wärme an die Umgebung abgeführt. Deswegen ist es nötig, dem System Energie zuzuführen, um diesen Wärmeverlust auszugleichen und eine konstante Manteltemperatur zu erhalten.

Die Überprüfung der Temperaturen ist allerdings nur an zwei Stellen möglich. Die Reaktion wird so durchgeführt, dass die Manteltemperatur der Reaktionstemperatur entspricht. 


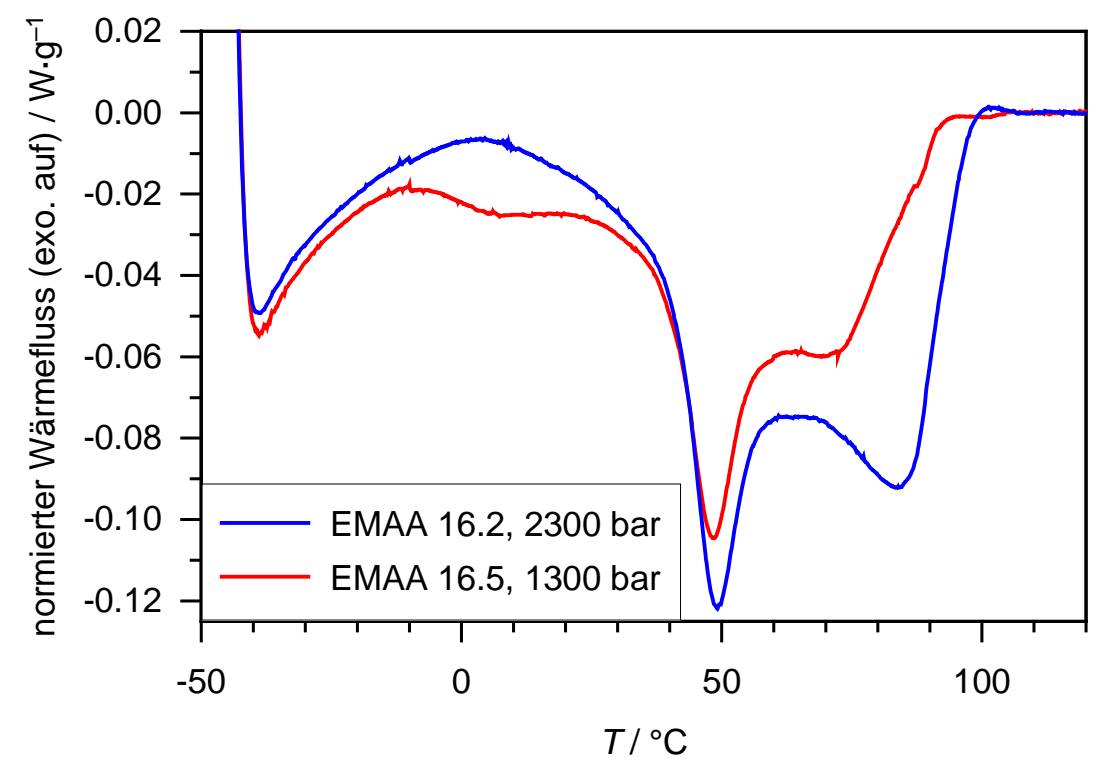

Abbildung 4.28: DSC-Thermogramm für EMAA 16.2, 2300 bar, $F_{M A A}=0.082$, EMAA 16.5, 1300 bar, $F_{M A A}=0.079$.

Abbildung 4.28 zeigt den Vergleich einer Hoch- und einer Niederdruckprobe, die unter adiabatischen Bedingungen bei $240{ }^{\circ} \mathrm{C}$ hergestellt worden sind. Aufgrund der Reaktionsführung werden Temperaturgradienten im Reaktor minimiert. Somit können keine Temperaturgradienten im Reaktor für den zusätzlichen Übergang im Bereich von ca. $0{ }^{\circ} \mathrm{C}\left(T_{\mathrm{G} 2}\right)$ verantwortlich sein, da durch die adiabatische Reaktionsführung nur minimale Temperaturunterschiede zwischen der Reaktionslösung und der Wand an den ausgewählten Messpunkten vorliegen. Auch der Monomerumsatz wird hier nur durch die Reaktionstemperatur bestimmt und weist keine Druckabhängigkeit auf, was Tabelle $4.2 \mathrm{zu}$ entnehmen ist. Der Abbildung 4.28 ist zu entnehmen, dass die Niederdruckprobe ein deutliches Minimum ( $\beta$-Minimum) im Bereich von $5{ }^{\circ} \mathrm{C}$ besitzt. Somit hat der Synthesedruck offensichtlich einen Einfluss auf die Ausbildung eines zusätzlichen Signals.

\subsubsection{Synthesetemperaturvariation bei der Copolymerherstellung}

Für eine bei sehr hohen Temperaturen von bis zu $300{ }^{\circ} \mathrm{C}$ synthetisierte Probenreihe sind die Ergebnisse der DSC-Analyse in Abbildung 4.29 zusammengetragen. Hier ist zu erkennen, dass eine bei 2300 bar hergestellte Probe eine geringe Abweichung vom erwarteten Verhalten einer Hochdruckprobe zeigt, siehe auch Kapitel 5.2.1. Es bildet sich bereits hier ein schwach zu erkennendes $\beta$-Minimum aus. Solche Beobachtungen ließen sich bei niedrigeren Synthesetemperaturen nicht feststellen. Die Niederdruckprobe bei 
1300 bar unterscheidet sich sehr deutlich von der Hochdruckprobe. Das $\beta$-Minimum ist für den hier verwendeten Säurefluss $\left(f_{\mathrm{MAA}}=0.010, \quad F_{\mathrm{MAA}}=0.035\right.$ bis 0.036$)$ sehr stark ausgebildet. Bei einem Synthesedruck von 1000 bar ist die Ausbildung noch stärker.

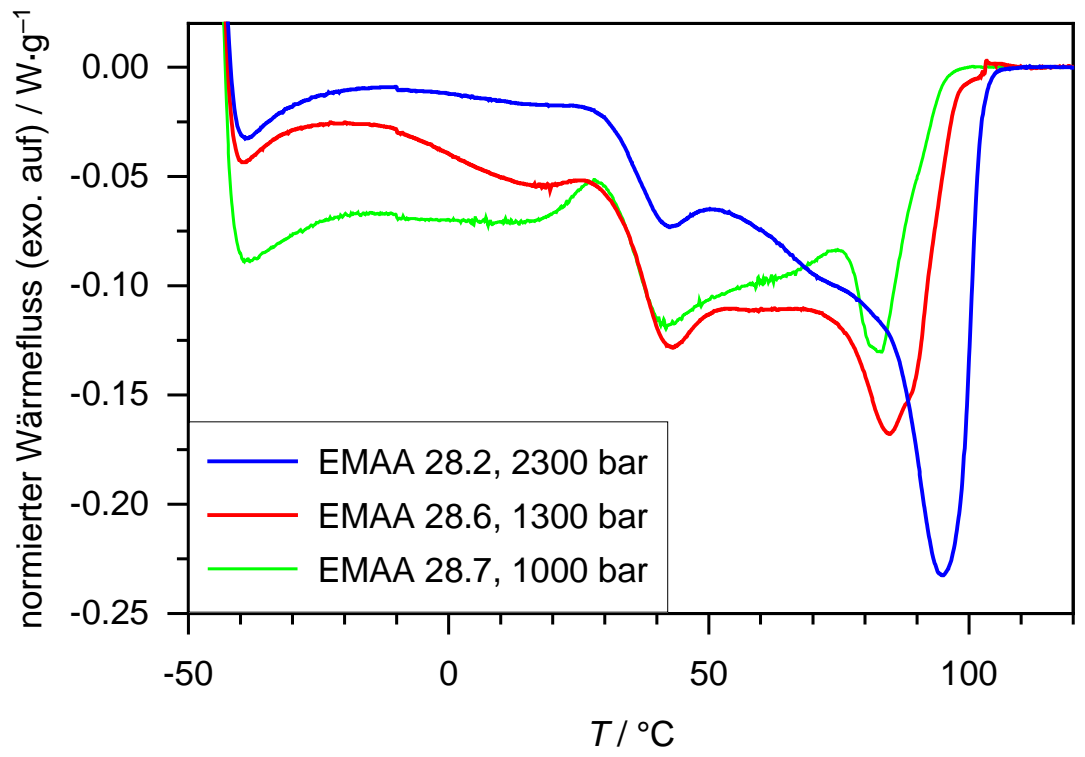

Abbildung 4.29: DSC-Thermogramm für EMAA 28.2, 2300 bar, $F_{M A A}=0.035$, EMAA 28.6, 1300 bar, $F_{M A A}=0.036$, EMAA 28.7, 1000 bar, $F_{M A A}=0.036$.

Aus diesen Beobachtungen und unter Berücksichtigung der bereits zuvor durchgeführten DSC-Messungen kann ein Synthesetemperatureinfluss auf die Ausbildung eines $\beta$ Übergangs vermutet werden. Es lag nahe, eine Synthesetemperaturvariation bei konstantem Säurefluss vorzunehmen und die daraus resultierenden Niederdruckproben zu vergleichen. Einer solcher Vergleich ist in Abbildung 4.30 gezeigt.

Der Übersichtlichkeit halber wird nur der Teilbereich des Thermogramms gezeigt, der den $\beta$-Übergang aufweist. Über die Veränderung der Lage des Minimums lässt sich aufgrund des breiten Übergangsbereichs nur schwer eine Aussage treffen. 


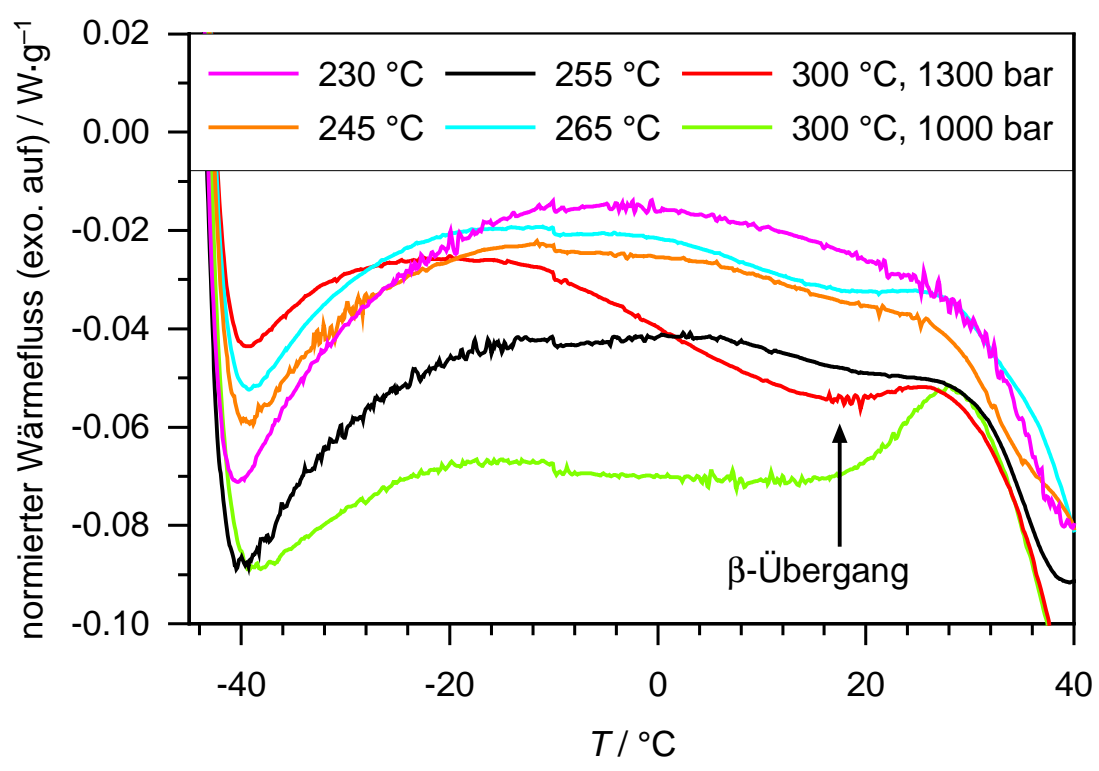

$\begin{array}{cccc}\text { Abbildung 4.30: } \text { DSC-Thermogramm } & \text { für } & \text { Niederdruckproben } & \left(f_{M A A}=0.010\right) \text {; } \\ \text { EMAA 33.8, } & 230^{\circ} \mathrm{C}, & 1400 \mathrm{bar}, & F_{M A A}=0.062 ; \\ \text { EMAA 27.8, } & 245^{\circ} \mathrm{C}, & 1400 \mathrm{bar}, & F_{M A A}=0.045 ; \\ \text { EMAA 32.3, } & 255^{\circ} \mathrm{C}, & 1300 \mathrm{bar}, & F_{M A A}=0.060 ; \\ \text { EMAA 25.6, } & 265^{\circ} \mathrm{C}, & 1300 \mathrm{bar}, & F_{M A A}=0.051 ; \\ \text { EMAA 28.6, } & 300^{\circ} \mathrm{C}, & 1300 \mathrm{bar}, & F_{M A A}=0.037 \text {, } \\ \text { EMAA 28.7, } & 300{ }^{\circ} \mathrm{C}, & 1000 \mathrm{bar}, & F_{M A A}=0.036 .\end{array}$

Bei tiefen Synthesetemperaturen ist nur eine geringe Ausbildung eines $\beta$-Minimums im Bereich von $0{ }^{\circ} \mathrm{C} \mathrm{zu}$ erkennen. Bei Temperaturerhöhung kommt es verstärkt zur Ausbildung dieses Minimums und bei einer Synthesetemperatur von $300{ }^{\circ} \mathrm{C}$ ist es am stärksten ausgebildet. Die Synthesetemperatur kann somit einen Einfluss auf die Ausbildung des zusätzlichen Übergangs, $T_{\mathrm{G} 2}$, haben. Dieser Temperatureffekt, der scheinbar auf Verzweigungen im Copolymer beruht, wird ausführlich in Kapitel 5.2.1 diskutiert. Bei den beiden Niederdruckproben, die bei einer Temperatur von $300{ }^{\circ} \mathrm{C}$ hergestellt wurden, ist ein signifikanter Unterschied festzustellen. Die Probe, die bei 1300 bar synthetisiert wurde, zeigt einen $\mathrm{zu}$ den bei niedrigeren Temperaturen hergestellten Polymeren ähnlichen Verlauf. Vergleicht man zum Beispiel die bei 1300 bar, $300{ }^{\circ} \mathrm{C}$ mit der bei 1300 bar und $265^{\circ} \mathrm{C}$ hergestellten Probe, so fällt auf, das lediglich die Intensität des Minimums bei der höheren Temperatur größer ist. Bei Betrachtung der dazugehörigen 1000 bar Probe, die nur bei sehr hoher Temperatur zu erhalten war, ist zu erkennen, dass sich die Kurvenform verändert. Das Minimum zeigt sich nun eher in der Form eines Plateaus und nach dessen Abschluss kommt es zu einem reproduzierbaren exothermen Übergang. Der weitere Kurvenverlauf nach dem Glasübergang, also bei höheren Temperaturen, ergibt ebenfalls einen exothermen Anstieg (nicht gezeigt). 
Eventuell ist hier die Kalibrierung auf die Schmelzwärme, wie oben beschrieben, problematisch und führt $\mathrm{zu}$ einer Verdrehung des gesamten Thermogramms. Abweichungen können durch das Material erklärt werden. Es ist zu erwarten, dass erhebliche Änderungen in den Syntheseparametern, wie zum Beispiel die Wahl einer deutlich höheren Synthesetemperatur, auch zu Änderungen im Schmelzverhalten der Polymere führen können. Ein weiterer Einfluss geht von der eingewogenen Menge aus. Werden große Mengen an Polymer eingewogen, so kann es zu einer schlechten Wärmeankopplung des Polymers an den Probentiegel kommen. Um diesen Effekt genauer $\mathrm{zu}$ erfassen, wurden Messungen an ein und demselben Polymer unter Variation der Einwaage durchgeführt. Es stellte sich heraus, dass die optimale Einwaage ca. $15 \mathrm{mg}$ beträgt. Bei höherer Einwaage kam es in einigen Fällen zu Abweichungen von den erwarteten Kurvenverläufen, es konnte allerdings kein eindeutiger Trend aufgezeigt werden. Die Verwendung von geringeren Probenmengen führte zum Erreichen der Auflösungsgrenze der Apparatur. Das äußerte sich in einem zackenförmigen Verlauf des Thermogramms. Die bei hohen Temperaturen hergestellten Proben weisen aufgrund des Abscheidevorgangs eine höhere Dichte auf. Gerade die Niederdruckproben liegen nach dem Abscheidevorgang auf Umgebungsdruck als viskose Flüssigkeit vor, die nach Abkühlung in einer kompakten Form erstarrt. Bei niedrigeren Synthesetemperaturen und höheren Synthesedrücken werden die Polymere in eine Art Krümel zerrissen und weisen eine erheblich geringere Dichte auf. Somit wird bei Hochtemperaturproben bei Verwendung eines gleichgroßen Volumenanteils ein höherer Massenanteil eingewogen. Reduziert man den Massenanteil auf die „optimale“ Menge von $15 \mathrm{mg}$, so liegt hier aufgrund der hohen Dichte eine geringe Oberfläche vor. Die Wärmeankopplung an die Wände des Probentiegels ist somit schlechter. Das kann zu einer Verschiebung der Schmelzlinie führen, auf die kalibriert wird. Genauere Informationen liefert das unkorrigierte Thermogramm. Hier wird keine Kalibrierung auf den Schmelzvorgang vorgenommen. In Abbildung 4.31 ist ein entsprechender Verlauf der DSC-Kurve für eine bei 1000 bar und $300{ }^{\circ} \mathrm{C}$ hergestellte Probe gegeben. Um sicherzustellen, dass speziell dieses Ergebnis reproduzierbar ist, wurde eine erneute Messung durchgeführt. Bei der ersten Messung (rote Kurve) wurde eine Einwaage von $24.0 \mathrm{mg}$ verwendet. Hierbei ist deutlich die Bildung des oben beschriebenen Plateaus zu sehen. Das Plateau wird durch einen exothermen Peak abgeschlossen. Bei der Wiederholungsmessung wurde ein nahezu gleicher Verlauf beobachtet. Aufgrund der geringeren Probenmasse von $17.3 \mathrm{mg}$ wird hier weniger Energie im Vergleich zur ersten Messung benötigt. Die Peakintensitäten sind 
generell geringer, aber auch hier ist ein exothermer Anteil zu beobachten. Somit kann ausgeschlossen werden, dass der exotherme Anteil durch die Normierung auf die Probenmasse zu Stande gekommen ist. Stellt man sich den hypothetischen Kurvenverlauf für eine rein statistische Probe vor (blau gestrichelt), so könnte dieser exotherme Bereich erklärt werden. Es handelt sich hier um den Beginn des Glasübergangs. Der $\beta$-Übergang ist bei diesen Synthesebedingungen stark ausgebildet. Das kann durch Verzweigungen im Copolymer erklärt werden, deren Häufigkeit mit steigender Synthesetemperatur zunimmt (siehe Kapitel 5.2.1).

Interessant wären weitere Proben, die bei noch höheren Synthesetemperaturen erhalten werden. Der apparative Aufbau lässt keine höheren Temperaturen zu.

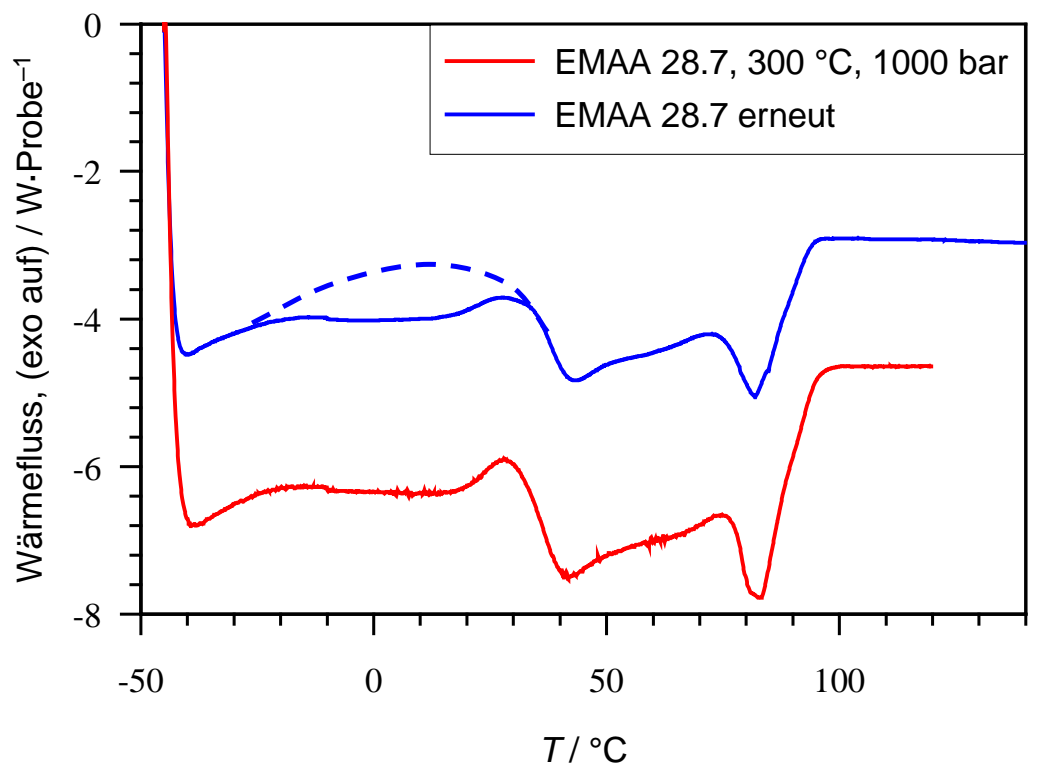

Abbildung 4.31: DSC-Rohdaten eines bei $300{ }^{\circ} \mathrm{C}$ und 1000 bar hergestellten Copolymers: rote Kurve: EMAA 28.7, erste Messung, Einwaage $24.0 \mathrm{mg}$, blaue Kurve: EMAA 28.7, zweite Messung, Einwaage $17.3 \mathrm{mg}$.

Für die Unterscheidung von statistisch und nicht-statistisch aufgebauten Polymere können die Copolymerproben, die bei $300{ }^{\circ} \mathrm{C}$ synthetisiert wurden trotzdem herangezogen werden, da hier ebenfalls ein signifikanter Unterschied zwischen den Hochdruck- und den Niederdruckproben besteht. 


\subsection{4 Überprüfung der Reproduzierbarkeit der mittels DSC beobachteten Relaxationen im Copolymer}

Um die Effekte der thermischen Vorgeschichte zu untersuchen, sind zwei Proben der MAA-reichen Serie EMAA 3, welche bei sehr unterschiedlichen Drücken synthetisiert wurden, in mehreren Stufen aufgeheizt worden (Abbildung 4.32 und Abbildung 4.33). Durch das Verpressen der gefüllten Probenpfännchen wird eine anfängliche, makroskopische Deformation des Probenmaterials erzeugt. Somit sollte ein wiederholtes Durchlaufen von Relaxationen, die zu einem Erweichen der Probe führen und irreversible Anteile haben, Unterschiede im Vergleich zum ersten Durchlauf aufweisen. Auch der Abscheidevorgang während der Synthese kann zu Unterschieden in der thermischen Vorgeschichte der Copolymere führen. Somit ist es möglich, dass bei tieferen Temperaturen liegende Relaxationen beeinflusst werden.

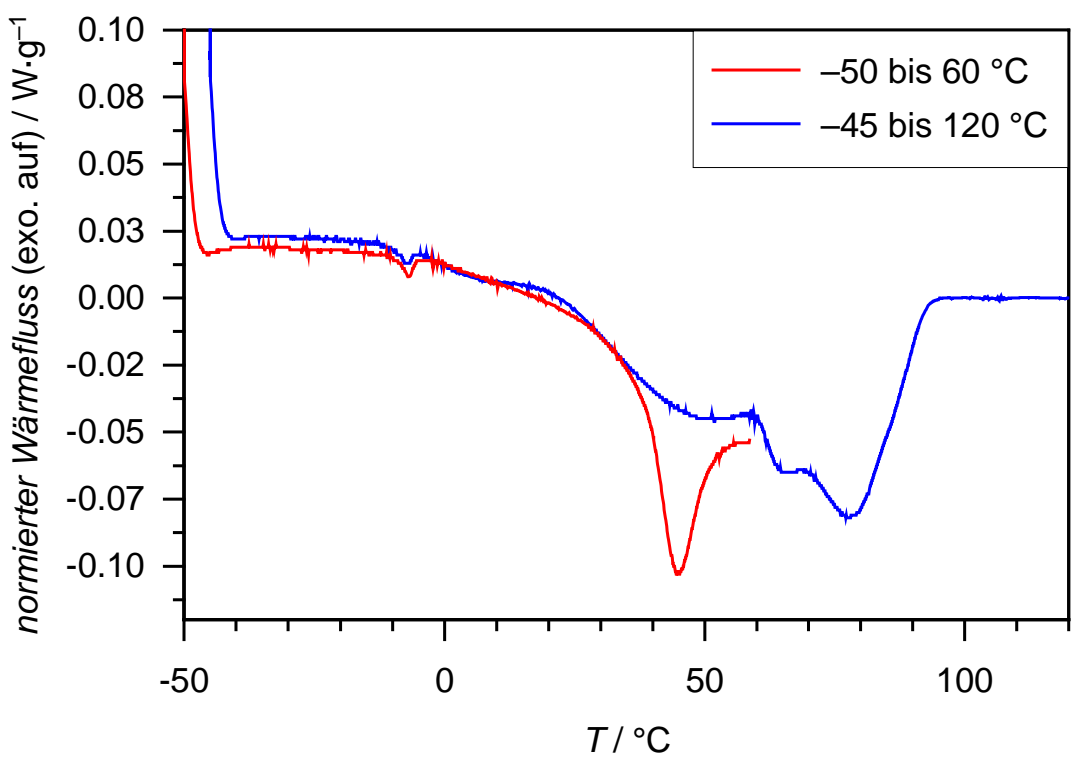

Abbildung 4.32: Stufenweises Aufheizen einer bei 2300 bar synthetisierten Copolymerprobe (EMAA 3.4, 2300 bar). ${ }^{[1]}$

Der in Abbildung 4.32 gezeigte Aufheizvorgang von -50 bis $60{ }^{\circ} \mathrm{C}$ mit einer Heizrate von $5{ }^{\circ} \mathrm{C} \cdot \mathrm{min}^{-1}$ zeigt, dass nach einmaligem Überschreiten des Glasübergangs, $T_{\mathrm{G}}$, sich die Form und Intensität des $\alpha$-Übergangs beim erneuten Heizvorgang von -45 bis $120{ }^{\circ} \mathrm{C}$ erheblich ändert. Während des Glasübergangs kommt es zu einer Neuorganisation des Materials, die nicht vollständig reversibel ist. Die Lage des Glasübergangs wird nicht signifikant beeinflusst. Der Kurvenverlauf bei Temperaturen bis $30{ }^{\circ} \mathrm{C}$ ist weitestgehend bei beiden Heizvorgängen gleich. Ein scharfes Maximum bei $-7^{\circ} \mathrm{C}$ ist $\mathrm{zu}$ beobachten. 
Dieser endotherme Ausschlag ist reproduzierbar bei allen an diesem Tag gemessenen Proben zu finden. Das kann möglicherweise auf das Vorhandensein von Wasser im oder am Referenztiegel zurückgeführt werden. Ein Austausch des Referenztiegels und die Verwendung von Stickstoff als Spülgas für die Probenkammer führte zur Eliminierung des Signals.

Für eine Niederdruckprobe wurde ähnlich verfahren, wie Abbildung 4.33 zeigt. Hier wurde eine Unterteilung in vier Aufheizschritte vorgenommen.

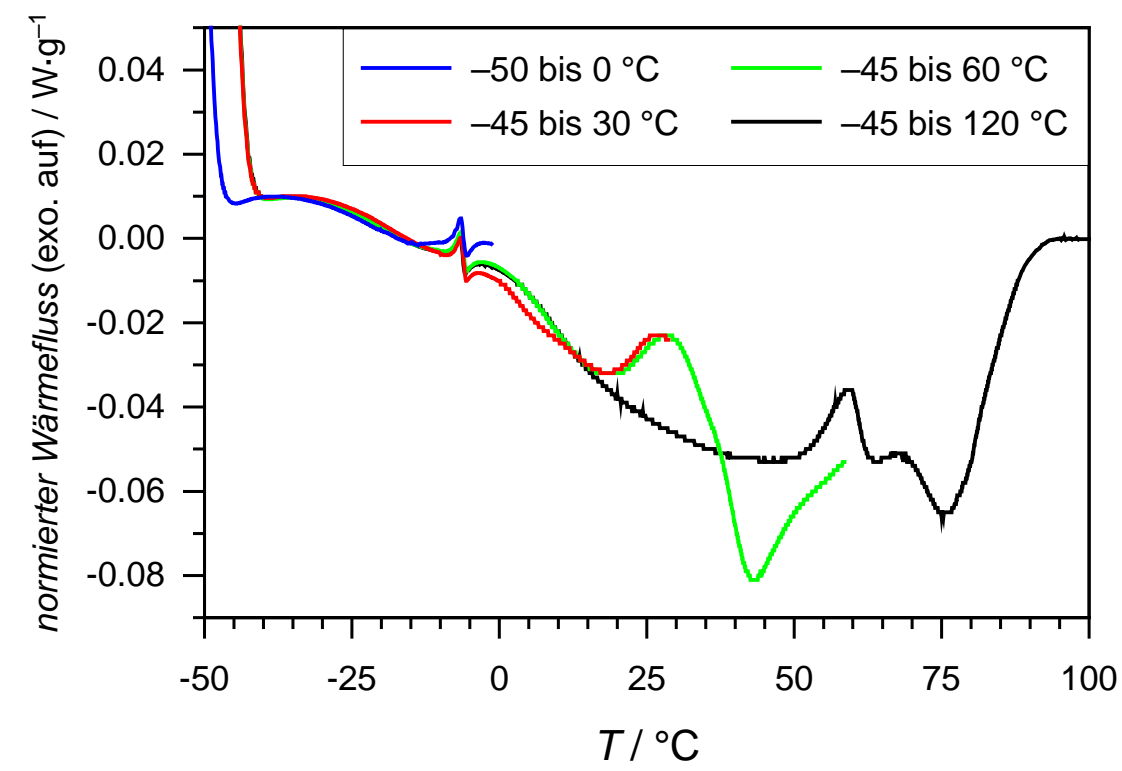

Abbildung 4.33: Stufenweises Aufheizen einer bei 1300 bar synthetisierten Copolymerprobe (EMAA 3.12, 1300 bar). ${ }^{[1]}$

Das oben beschriebene Artefakt befindet sich hier ebenfalls im Bereich von $-7^{\circ} \mathrm{C}$, bedarf aber keiner weiteren Beachtung. Der Aufheizvorgang wurde zum ersten Mal bei $0{ }^{\circ} \mathrm{C}$ unterbrochen um eine eventuelle Entspannung bei diesen tiefen Temperaturen zu beobachten. Auch der Übergang über das $\beta$-Minimum, das nur die Niederdruckprobe aufzeigt, führt zu keiner Veränderung des Verlaufs des Wärmeflusses. Zu Veränderungen im Wärmefluss kommt es erst, wenn der Glasübergang, $T_{\mathrm{G}}$, im vorangegangenen Heizzyklus überschritten wurde. Dies führt dann zu einem breiteren Signal beim folgenden Aufheizen, welches das $\beta$-Minimum sogar überdecken kann. Darüber hinaus ist vor dem Erreichen des Signals für den Schmelzvorgang ein weiteres Minimum zu erkennen. Ob sich dabei das Signal für den Schmelzübergang $\left(T_{\alpha 2}\right)$ oder das für den Glasübergang $\left(T_{\alpha}\right)$ in zwei Vorgänge aufspaltet, ist schwer zu beurteilen. 


\subsubsection{Diskussion der DSC-Messungen}

Die Thermogramme der Homopolymere PE und PMAA (Abbildung 4.24) zeigen im beobachteten Wärmefluss nur wenige Übergänge im Temperaturbereich bis $150{ }^{\circ} \mathrm{C}$ auf. Die in dieser Arbeit synthetisierten Proben weisen besonders im Falle von Niederdruckproben deutlich mehr Struktur auf. Anhand des beschriebenen zusätzlichem Peaks, dem $\beta$-Minimum für einen weiteren Übergang $T_{\mathrm{G} 2}$ kann eine Unterscheidung von statistisch (Hochdruck) und nicht-statistischen (Niederdruck) Proben vorgenommen werden.

Aufgrund der fehlenden Stufen oder Minima im Thermogramm für PE kann ausgeschlossen werden, dass es sich beim $\beta$-Minimum um eine für PE charakteristische Kurbelwellenbewegung handelt (siehe Kapitel 4.9.1). Diese wurde bei tieferen Temperaturen bei entsprechenden DMA-Messungen gefunden. Auffällig ist die verstärkte Ausprägung des $\beta$-Minimums bei mit niedrigem Synthesedruck hergestellten Proben und dass es mit steigendem MAA-Gehalt anwächst. Dies sind deutliche Indizien dafür, dass bei niedrigerem Druck synthetisiertes EMAA über zusätzliche Relaxationsmoden verfügt. Studien an reinem PE mit niedriger Dichte haben gezeigt, dass die Anzahl der Verzweigungen nur sehr schwach druckabhängig ist und somit nicht ausreicht, um die beobachteten Phänomene zu erklären ${ }^{[18,19]}$.

Die Intensität des $\alpha 2$-Minimums nimmt mit steigendem MAA-Gehalt ab. Proben, die bei niedrigem Synthesedruck hergestellt wurden, weisen eine geringere Intensität des Minimums auf als die Hochdruckproben. Dies ist dadurch zu erklären, dass eine Erhöhung des MAA-Anteils die Bildung von kristallinen PE-Bereichen im EMAA erschwert, insbesondere wenn diese in „Blöcken“, bestehend aus zwei oder mehr MAAMonomereinheiten, auftreten. Dies führt zu einem höheren Anteil von amorphen Bereichen, wodurch das $\alpha$-Minimum in vielen Fällen an Intensität gewinnt.

Die verstärkte Bildung von amorphen Bereichen bei Niederdruckproben kann in Abbildung 4.30 beobachtet werden. Die Intensität des Glasübergangs ist für Copolymere, die bei hoher Temperatur synthetisiert worden, deutlich höher im Vergleich zu bei niedrigeren Temperaturen hergestellten Proben.

Bei Untersuchungen, die parallel zu dieser Arbeit an Poly(Ethen-co-Acrylsäure) ausgeführt wurden, zeigen sich gute Übereinstimmungen zwischen den sich lediglich durch eine Methylgruppe unterscheidenden Comonomeren. Die Thermogramme der EAA-Proben zeigen ebenfalls die gleichen Tendenzen auf. Hier konnte eine Ausbildung eines $\beta$ Minimums für Niederdruckproben beobachtet werden. In Abbildung 4.34 ist ein 
Thermogramm für ein EAA-Copolymer dargestellt, was bei einem hohen Säuredosierstrom $\left(f_{\mathrm{AA}}=0.0180\right)$ hergestellt wurde. Hier spiegeln sich die für EMAA-Copolymere gemachten Beobachtungen wider. Weitere Ergebnisse finden sich bei Nergui. ${ }^{[20]}$

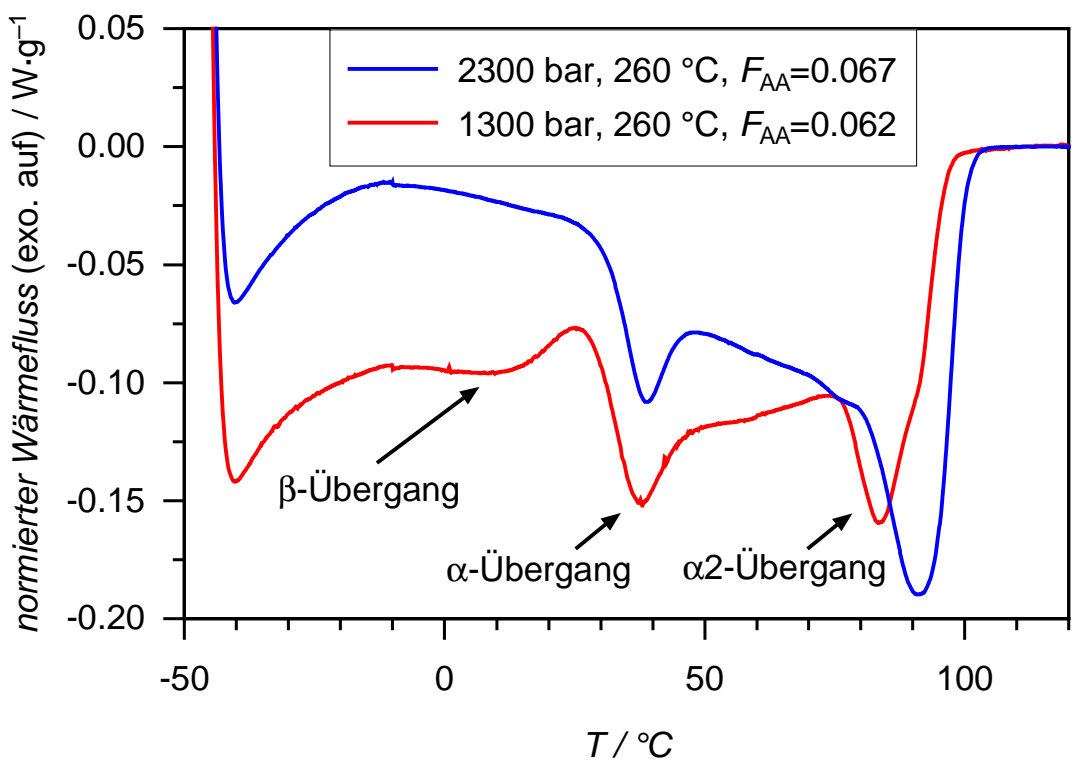

Abbildung 4.34: Thermogramm für Ethen-Acrylsäurecopolymere, die bei verschiedenen Synthesedrücken hergestellt wurden.

Für EAA kann ebenfalls anhand der DSC-Kurven eine Aussage über die Kristallinität der Proben gemacht werden. Die Hochdruckprobe, die bei 2300 bar hergestellt wurde, zeigt eine stärkere Ausbildung des Schmelzpeaks ( $\alpha 2)$ im Vergleich zu einer Probe, die nahe der Phasengrenze bei 1300 bar hergestellt wurde. Diese Beobachtungen stehen in guter Übereinstimmung mit den Ergebnissen, die für das System Ethen/Methacrylsäure vorgestellt wurden.

Um weitere Ergebnisse für die Kristallinität der Proben zu erhalten, wurden für EAA Röntgenstrukturanalysen durchgeführt.

In Abbildung 4.35 sind die Röntgenstrukturaufnahmen für eine Hoch- und eine Niederdruckprobe gegeben. Für die bei 2300 bar hergestellte Probe zeigen sich bei Braggwinkeln $(2 \Theta)$ von $25^{\circ}$ und $28^{\circ}$ scharfe Signale. Das wird durch eine teilkristalline Struktur der Proben verursacht. Kommt es zu einem breiten Signal in diesem Bereich, so spricht das für eine amorphe Struktur. Das ist hier der Fall für die Niederdruckprobe. Diese Erkenntnis deckt sich sehr gut mit den Aussagen über Unterschiede in der Kristallinität bei Hoch- und Niederdruckproben für EMAA die anhand der oben gezeigten DSC-Analysen belegt werden können. 


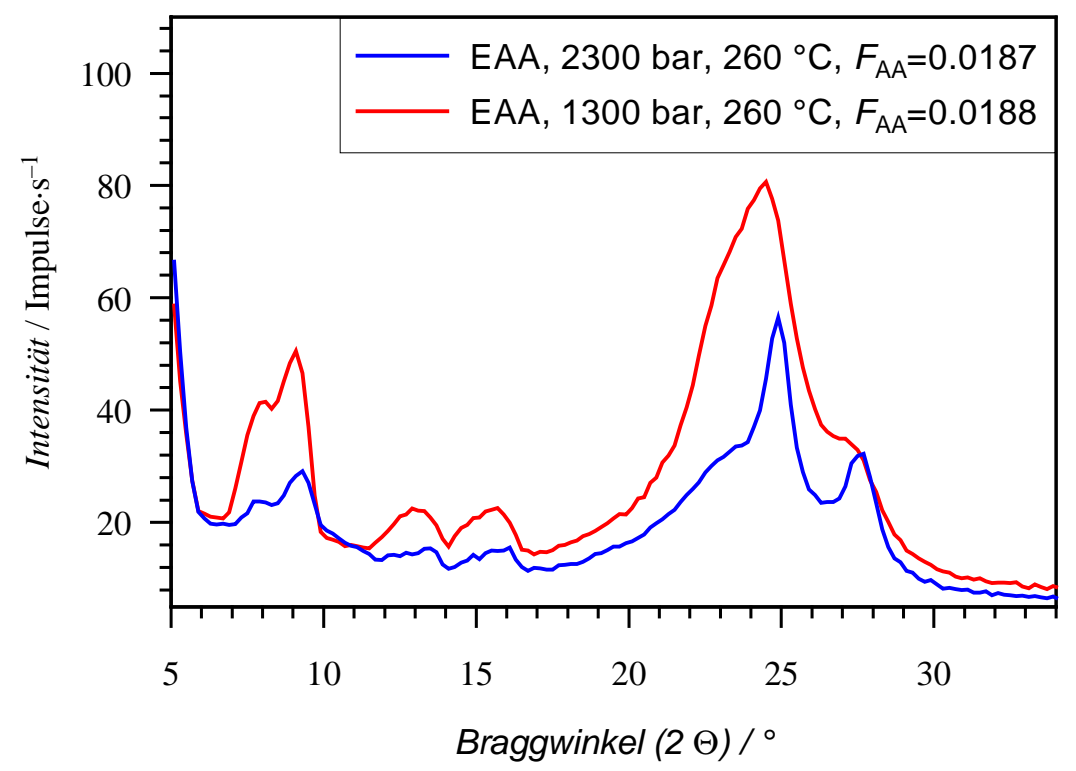

Abbildung 4.35: Röntgenstrukturanalyse von EAA-Copolymeren mit einem Säuregehalt von $F_{A A}=0.019$ bei unterschiedlichen Synthesedrücken hergestellt.

Zusammenfassend lässt sich festhalten, dass bereits die kalorimetrische Analyse Unterschiede zwischen den synthetisierten Proben aufzeigt. Bei niedrigerem Druck synthetisierte Proben, bei denen von einer weniger statistischen Verteilung von MAAEinheiten ausgegangen wird, sind deutlich ausgeprägte zusätzliche Relaxationen unterhalb der $\alpha$-Relaxation zu beobachten. Auch die $\alpha 2$-Relaxation ist druckabhängig. Eine Änderung der mikroskopischen Eigenschaften hat also zu einer deutlichen Änderung der makroskopischen, kalorimetrischen Eigenschaften geführt. Somit kann bereits hier geschlossen werden, dass sich diese Effekte auch in den mechanischen Eigenschaften widerspiegeln sollten. 


\subsection{Dynamisch-mechanische Analyse}

Aufgrund der erheblich unterschiedlichen Eigenschaften der Hoch- und Niederdruckproben, die sich bereits in den kaloriemetrischen Messungen gezeigt haben, ist ebenfalls bei der mechanischen Spektroskopie ein detektierbarer Unterschied zu erwarten. In diesem Kapitel werden die Ergebnisse der DMA-Messungen diskutiert.

\subsubsection{Effekte durch Probenpräparation}

Wie bereits in Kapitel 4.10 beschrieben, werden einige Strukturübergange im Polymeren durch die thermische Vorgeschichte der Proben beeinflusst. Im Falle der DMA-Messungen wurden einige Proben, die mittels des Pressverfahrens, beschrieben in Kapitel 3.2.3.4, hergestellt wurden, einem mehrmaligen Heizvorgang unterworfen, der im Folgenden beschrieben wird. Das typische Experiment beginnt mit dem Probeneinbau bei Raumtemperatur. Der Probenzylinder wird dann mit einer möglichst geringen statischen Kraft fixiert und in den Heizofen eingebaut, der von einer Kühlungsvorrichtung (Block, gespült mit flüssigem Stickstoff) umgeben ist. Nun beginnt der mehrstündige Kühlvorgang bis zu Probentemperaturen von $-135^{\circ} \mathrm{C}$. Nach Erreichen einer konstant tiefen Blocktemperatur kann der kontrollierte Heizvorgang gestartet werden. Nach dem ersten Heizvorgang mit einer Heizrate von $5^{\circ} \mathrm{C} \cdot \mathrm{min}^{-1}$ bis $\mathrm{zu}$ Temperaturen im Bereich des Übergangs in die Flüssigkeit $(\alpha 2)$ bei ca. $90^{\circ} \mathrm{C}$ kann unter verschiedenen Bedingungen eingekühlt werden. Es gibt die Möglichkeit mit angelegten Kräften, also unter Kompression, einzukühlen und auch das Ablösen des Probekörpers von dem Quarzgestänge hat Einfluss auf den darauffolgenden Heizvorgang. Diese Einflüsse spiegeln sich besonders in den Absolutwerten des Speicher- und des Verlustmoduls wieder. Eine unterschiedlich große Auflagefläche der Probe hat einen großen Einfluss auf die Module und auch die Vorkompression äußert sich in den erlangten Werten. Eine ausführliche Auswertung bezüglich dieser Effekte findet sich bei Hachenberg. ${ }^{[1]}$ Eine praktikable Lösung für den direkten Vergleich von Hoch- und Niederdruckproben bietet die Auswertung über den Phasenwinkel $\tan \delta$. Diese Messgröße, die auf der Phasenverschiebung zwischen Anregung und Reaktion der Probe beruht, ist nicht von der Probengeometrie abhängig und wird auch nicht von den Absolutwerten der Module beeinflusst. 


\subsubsection{Existenz zusätzlicher Relaxationen bei Niederdruckproben}

Die Ergebnisse der DSC-Analysen legen nahe, dass Proben, die im Bereich der Phasengrenze synthetisiert wurden, zusätzliche Relaxation zeigen. Diese werden im Temperaturbereich unterhalb des Glasübergangs erwartet (siehe Kapitel 4.5.1.2). Um später eine bessere Zuordnung der Relaxationsprozesse treffen zu können, wurden zuerst Homopolymere der beiden verwendeten Monomere vermessen. Für die PE-Messung konnte ein Teil des Probenmaterials in einem kleinen Schnappdeckelglas vorsichtig aufgeschmolzen werden und anschließend ein zylinderartiger Körper ausgestanzt werden. Die Grundfläche wurde für eine optimale Auflage mittels eines Skalpells begradigt. Die vermessene Probe zeigt im Verlauf des $\tan \delta$ ein ausgeprägtes Maximum bei $-118^{\circ} \mathrm{C}$. Darüber hinaus ist der Abbildung 4.36 ein sehr scharfes Maximum bei $-45^{\circ} \mathrm{C} \mathrm{zu}$ entnehmen. Dieses Maximum beruht auf dem Platzen eines Kühlschlauches aus Silikon und ist keine Relaxation des Materials sondern eine Erschütterung der Apparatur. Bei darauffolgenden Messungen wurden die Kühlschläuche durch Metallgewebeschläuche ersetzt. Ansonsten zeigen sich bei der logarithmischen Auftragung des Phasenwinkels gegen die Temperatur keine signifikanten Relaxationen.

Bei der Probenpräparation von PMAA ergaben sich erhebliche Probleme. Ein Aufschmelzen des Polymers war ohne Zersetzung des Materials nicht möglich (siehe Kapitel 4.10.1). Bei dem Säurehomopolymer handelt es sich um ein wasserlösliches Polymer und nach Wasserzugabe konnte aus einer gelartigen Flüssigkeit ein Probenkörper geformt werden. Mittels einer Vakuumpumpe wurde ein Großteil des Wassers aus dem Probenkörper entfernt und es ergab sich ein verwendbarer Probenkörper, der allerdings aufgrund seiner Kristallinität eine hohe Zerbrechlichkeit aufwies. Der gemessene Phasenwinkel zeigt, im Gegensatz zu PE, keine eindeutigen Übergänge auf. Es kann lediglich eine breite Schulter im Temperaturbereich von $-45^{\circ} \mathrm{C}$ beobachtet werden.

Die verwendeten Kräfte werden im Folgenden mit $s$ für die statische und mit d für die dynamische Kraft gekennzeichnet. 


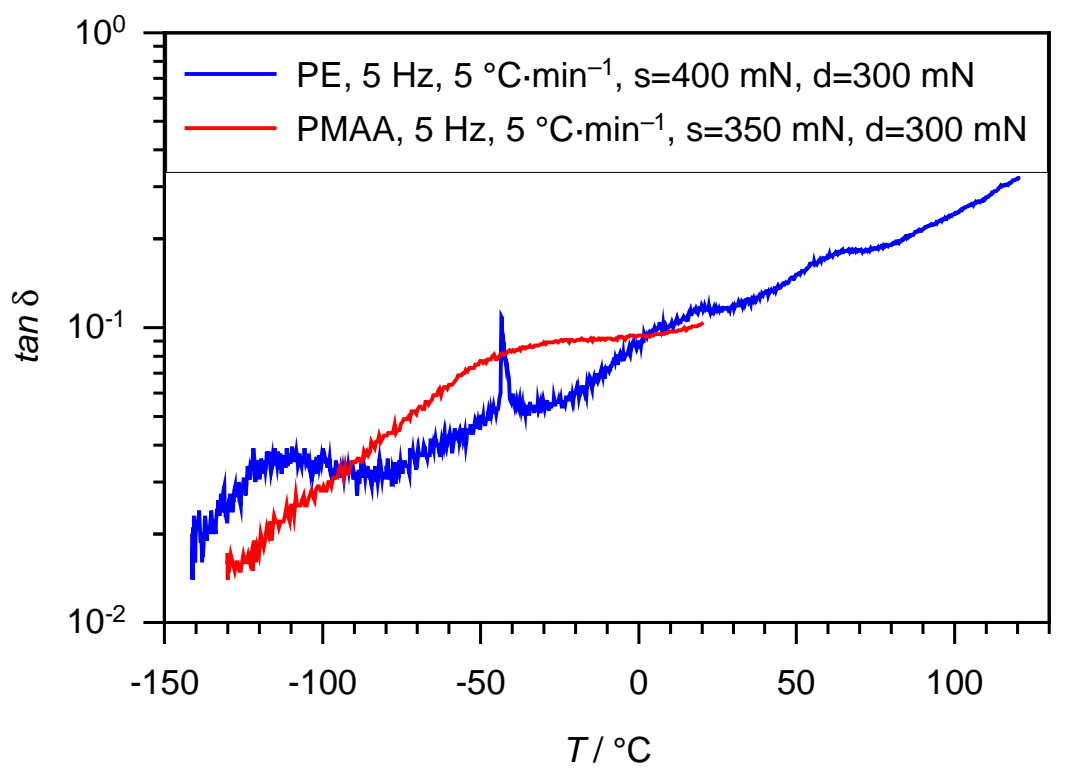

Abbildung 4.36: Verlauf des tan $\delta$ der mechanischen Spektroskopie an den Homopolymeren PE und mit Wasser versetztem PMAA. ${ }^{[1]}$

Hachenberg ${ }^{[1]}$ zeigt auf, dass in vielen Fällen Niederdruckproben besser zu messen sind. Einige Hochdruckproben weisen keinen zur Detektion ausreichenden Verlustmodul auf. Für die Hochdruckprobe EMAA 12.11 in Abbildung 4.37 ist bei Temperaturen unterhalb von $-45^{\circ} \mathrm{C}$ kein Phasenwinkel messbar. Für die Niederdruckprobe EMAA 12.4 zeigen sich aufgrund des geringen Säuredosierstroms von lediglich $5.3 \mathrm{~g} \cdot \mathrm{h}^{-1}$ nur geringfügige Unterschiede zur Hochdruckprobe. So kann zum Beispiel eine Schulter für EMAA 12.4 bei $-12{ }^{\circ} \mathrm{C}$ beobachtet werden, wo hingegen EMAA 12.11 nur eine Änderung in der Steigung des Phasenwinkels zeigt. 


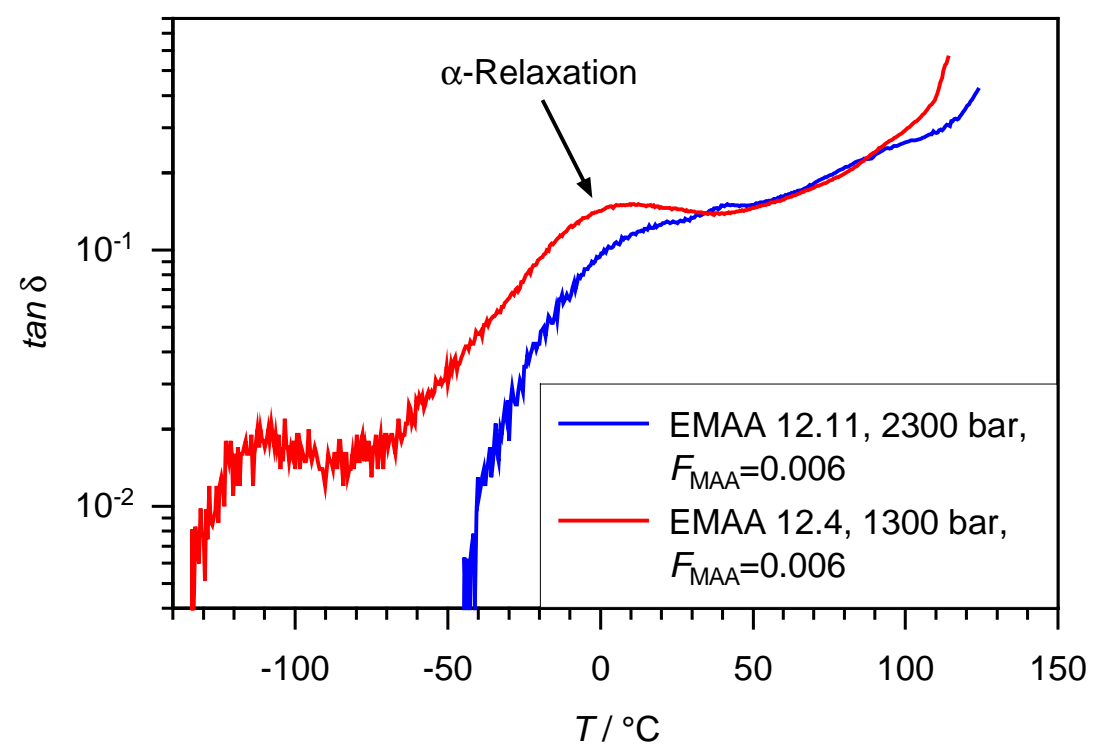

Abbildung 4.37: Verlauf des tan $\delta$ der mechanischen Spektroskopie von EMAACopolymeren mit einem niedrigem MAA-Gehalt bei $5 \mathrm{~Hz}, 5^{\circ} \mathrm{C} \cdot \mathrm{min}^{-1}$, $s=350 \mathrm{mN}, d=300 \mathrm{mN}{ }^{[1]}$

Bei Messungen an der Probenreihe EMAA 2 (mittlerer MAA-Dosierstrom) ist die Aufzeichnung des Phasenwinkels für Hoch- und Niederdruckproben jeweils über den gesamten gemessenen Temperaturbereich möglich, was Abbildung 4.38 belegt. Es zeigt sich bei beiden Proben eine Relaxation im Bereich von -80 bis $-150{ }^{\circ} \mathrm{C}$, die Maxima bei $-123{ }^{\circ} \mathrm{C}$ (EMAA 2.2) und $-115{ }^{\circ} \mathrm{C}$ (EMAA 2.10) besitzen. Die Niederdruckprobe weist darüber hinaus einen zusätzlichen Übergang mit einem Maximum bei $-54{ }^{\circ} \mathrm{C}$ auf.

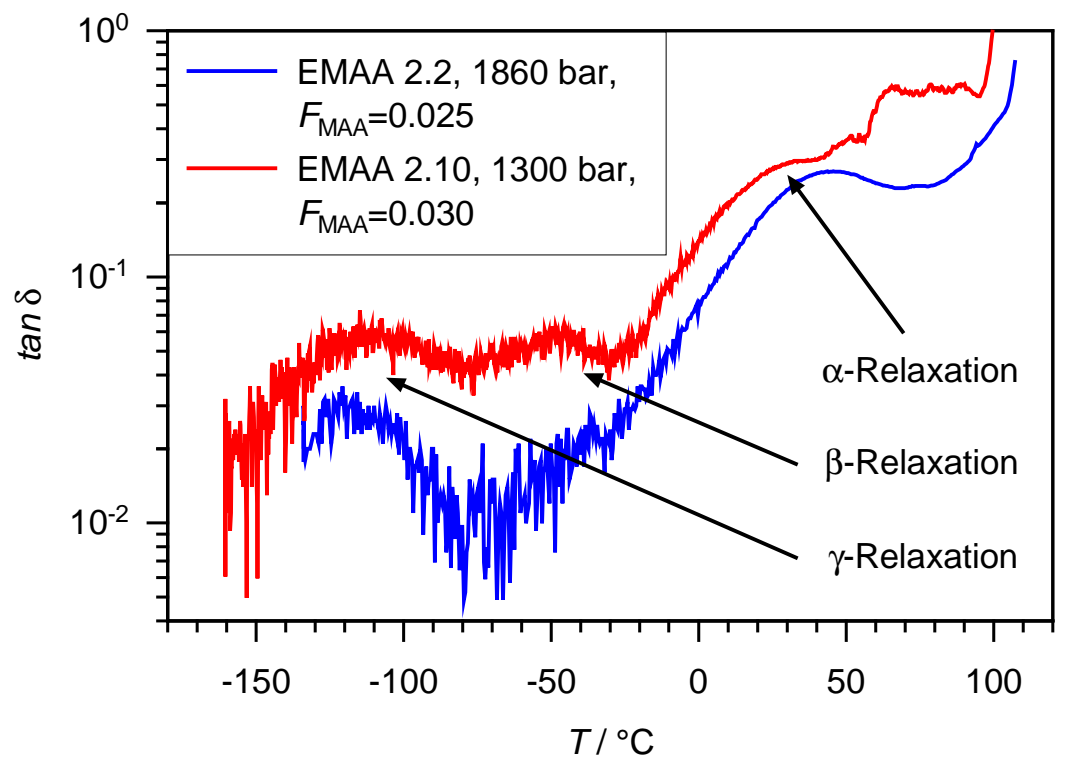

Abbildung 4.38: Verlauf des tan $\delta$ der mechanischen Spektroskopie von EMAA-Copolymeren mit einem mittleren MAA-Gehalt bei $5 \mathrm{~Hz}, 5^{\circ} \mathrm{C} \cdot \mathrm{min}^{-1}$, EMAA 2.2: $s=400 \mathrm{mN}, d=300 \mathrm{mN}$, EMAA 2.10: $\mathrm{s}=200 \mathrm{mN}, d=100 \mathrm{mN} .^{[1]}$ 
Hier kann gefolgert werden, dass sich der Übergang, der bei tiefen Temperaturen beobachtet wird ( -150 bis $-80^{\circ} \mathrm{C}$ ) auf Effekte durch die PE-Hauptkette zurückführen lässt. In diesem Bereich wurde auch ein Übergang für das Homopolymer PE beobachtet, wo hingegen das Homopolymer von PMAA dort keinen Übergang zeigt. Diese Bewegung kann auf die in Kapitel 4.9.1 beschriebene Kurbelwellenbewegung von mehreren Methyleneinheiten zurückgeführt werden. Diese Übergänge lassen sich bei vielen Messungen sowohl an der Hoch- als auch an der Niederdruckprobe beobachten. In einigen Fällen ist das Material allerdings zu hart, so dass dieser Übergang nur ansatzweise oder überhaupt nicht beobachtet werden kann. Die Relaxation bei ca. 30 bis $40{ }^{\circ} \mathrm{C}$ wird durch den Glasübergang ( $\alpha$-Übergang) verursacht. In diesem Temperaturbereich wurde der Glasübergang auch bei den DSC-Analysen beobachtet. Je nach Säuregehalt der Copolymere ist hier eine Lageveränderung des Maximums festzustellen. Für Proben, die einen hohen Anteil an Methacrylsäure im Copolymer eingebaut haben, liegt die Glastemperatur bei höheren Temperaturen als für Proben, die nur einen geringen Anteil an Methacrylsäureeinheiten aufweisen. EMAA 12.4 mit einem MAA-Gehalt von $F_{\mathrm{MAA}}=0.006$ zeigt zum Beispiel das Maximum für die $\alpha$-Relaxation bei Temperaturen im Bereich von $0{ }^{\circ} \mathrm{C}$. Der bei höheren Temperaturen folgende Übergang, der ab ca. $80^{\circ} \mathrm{C}$ zu beobachten ist, ist der Übergang in das viskose Fließen. Mit diesem Aufbau der DMA lässt sich das Verhalten bei höheren Temperaturen nicht analysieren. Der Probenkörper wird zusammengequetscht. Eine erneute Messung des Probenkörpers ist nur möglich, wenn der Heizvorgang ausreichend früh beendet wird, bevor es zu einer völligen Deformation der Probe kommt.

Nach anfänglichen Erfolgen mit einer Probenreihe, die einen mittleren Gehalt an MAAEinheiten aufweist (EMAA 2) wurden für Copolymere, die einen höheren Säureanteil aufweisen, deutlichere Effekte erwartet. Die in der DSC-Analyse die deutlichsten Unterschiede zwischen Hoch- und Niederdruckproben aufweisende Reihe EMAA 3 sollte auch in der DMA deutliche Unterschiede zwischen den bei verschiedenen Drücken hergestellten Proben zeigen. Das Resultat dieser Messungen ist in Abbildung 4.39 angegeben. Die Hochdruckprobe weist wiederum eine hohe Härte auf und ist nicht über den gesamten gezeigten Temperaturverlauf messbar. Lediglich bei $-100{ }^{\circ} \mathrm{C}$ zeigt sich hier ein Maximum, das durch die Hauptkette des Polymers verursacht wird. Ansonsten ist erst wieder ab einer Temperatur von $-10{ }^{\circ} \mathrm{C}$ ein Signal für den Phasenwinkel detektierbar. Die Niederdruckprobe zeigt ebenfalls die Kurbelbewegung der Hauptkette. Im Bereich um $-70{ }^{\circ} \mathrm{C}$ herum ist eine Schulter ausgeprägt. Diese Schulter kann mit dem bereits bei 
EMAA 2.10 beobachtetem zusätzlichem Maximum für Niederdruckproben in Verbindung gebracht werden. Eine Erklärung, warum es trotz hohem Säuregehalt des Copolymers nicht zur klaren Ausbildung eines Maximums kommt und warum die Lage dieses durch die Schulter angedeuteten Maximums im Vergleich zur Probe EMAA $2.10 \mathrm{zu}$ tieferen Temperaturen verschoben ist, wird weiter unten gegeben (Kapitel 4.11.3).

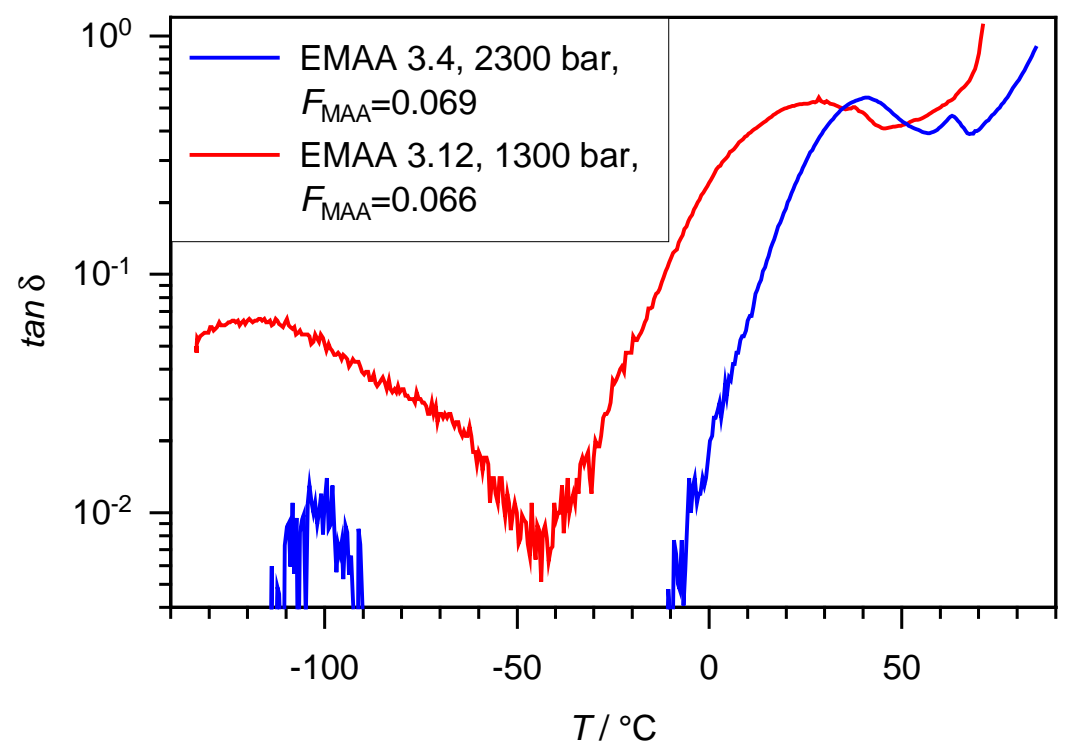

Abbildung 4.39: Verlauf des tan $\delta$ der mechanischen Spektroskopie von EMAACopolymeren mit einem hohem MAA-Gehalt bei $5 \mathrm{~Hz}, 5^{\circ} \mathrm{C} \cdot \mathrm{min}^{-1}$, $s=350 \mathrm{mN}, d=300 \mathrm{mN}{ }^{[1]}$

Um Umsatzeffekte für die Entstehung des zusätzlichen Maximums ausschließen zu können, wurden Experimente unter adiabatischen Bedingungen durchgeführt. Hier wird der Monomerumsatz nur durch die Reaktionstemperatur bestimmt. Durch die Übereinstimmung von Mantel und Reaktionstemperatur werden Temperaturgradienten innerhalb der Probe weitestgehend vermieden. Die Problematik dieser Reaktionsführung besteht im Auffinden von stabilen Betriebspunkten. Abbildung 4.40 zeigt die erhaltenen Ergebnisse der DMA-Messung für unter adiabatischen Bedingungen bei $260{ }^{\circ} \mathrm{C}$ hergestellte Polymerproben. Hier zeigt die Niederdruckprobe ebenfalls einen deutlich ausgeprägten zusätzlichen Peak im Bereich von $-50{ }^{\circ} \mathrm{C}$ im Vergleich zur Hochdruckprobe, der in Anlehnung an die DSC-Messungen ebenfalls als $\beta$-Peak bezeichnet wird. Hier weist das $\beta$-Maximum eine ähnliche hohe Intensität auf, wie der $\gamma$-Peak für die Hauptkettenbewegung. Aufgrund der adiabatischen Reaktionsführung können nun Umsatzeffekte oder Einflüsse durch starke Unterschiede zwischen Mantel- und Reaktionstemperatur weitestgehend ausgeschlossen werden. 


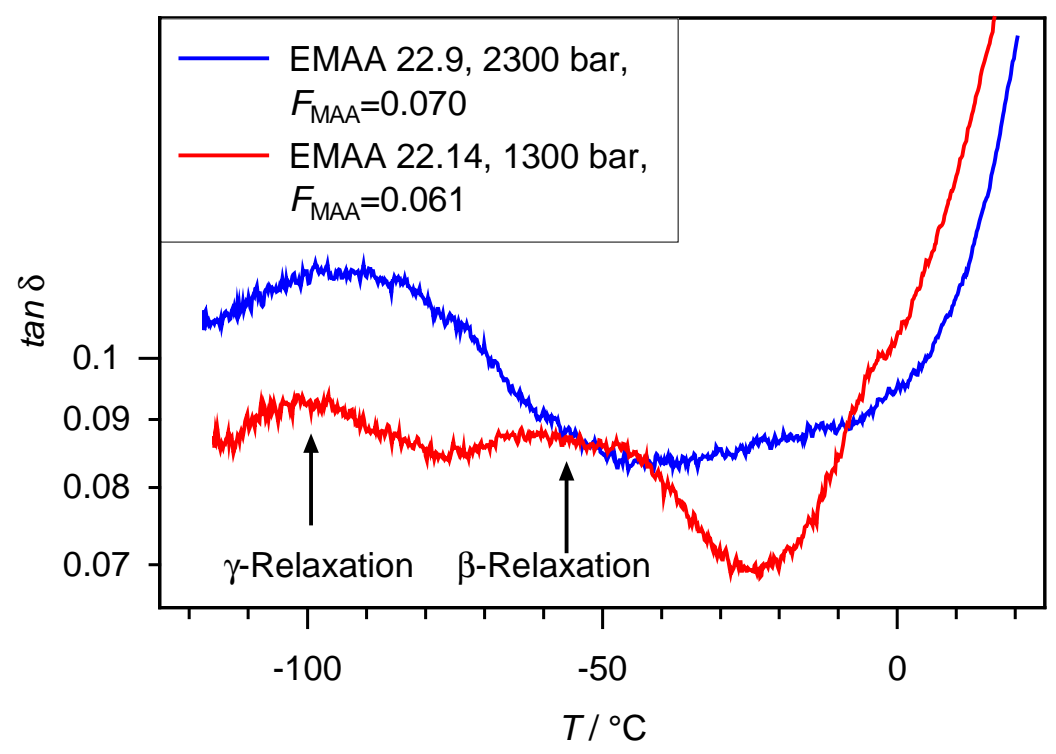

Abbildung 4.40: Verlauf des tan $\delta$ der mechanischen Spektroskopie von unter adiabatischen Bedingungen hergestellten EMAA-Copolymeren mit einem hohem MAA-Gehalt bei $5 \mathrm{~Hz}, 5^{\circ} \mathrm{C} \cdot \mathrm{min}^{-1}, \mathrm{~s}=500 \mathrm{mN}, d=400 \mathrm{mN}$.

Ein signifikanter Unterschied zwischen den gemessenen Probenreihen EMAA 3 und 22 besteht in der Höhe der verwendeten Kräfte. Für höhere Kräfte (EMAA 22) konnte eine deutliche Ausprägung des $\beta$-Maximums beobachtet werden. Aus diesem Grund wurde an der Probe EMAA 22.14 eine Variation der Kräfte vorgenommen.

\subsubsection{Variation von statischen und dynamischen Kräften an Niederdruck- proben}

Es wurde eine Variation der angelegten Kräfte vorgenommen. Die Erwartung war hierbei, dass die Intensität des Maximum kräfteabhängig ist. Dazu wurde die Probe EMAA 22.14 herangezogen, da sie sich aufgrund vorheriger Ergebnisse als sehr gut geeignet gezeigt hat und eine deutliche $\beta$-Relaxation zeigt. Die Messungen wurden immer am gleichen Probenkörper durchgeführt. Die Einkühlung erfolgte jeweils mit $\mathrm{s}=100 \mathrm{mN}$ und d=0 mN. Die verwendeten Kräfte wurden von niedrigen Kräften, wie zum Beispiel s=200 mN und $\mathrm{d}=100 \mathrm{mN}$ bis hin zu hohen Kräften von $\mathrm{s}=900 \mathrm{mN}$ und d=800 mN variiert. Die Wahl der Kräfte erfolgte in zufälliger Reihenfolge. Die Messungen sind reproduzierbar, so dass Alterungseffekte der Proben durch das Messen selbst ausgeschlossen werden können. Die Ergebnisse zeigen Abbildung 4.41 und Abbildung 4.42. Der Übersichtlichkeit halber 
wurden jeweils kleinere und jeweils höhere Kräfte im Vergleich zu Abbildung 4.40 in einer Abbildung zusammengefasst.

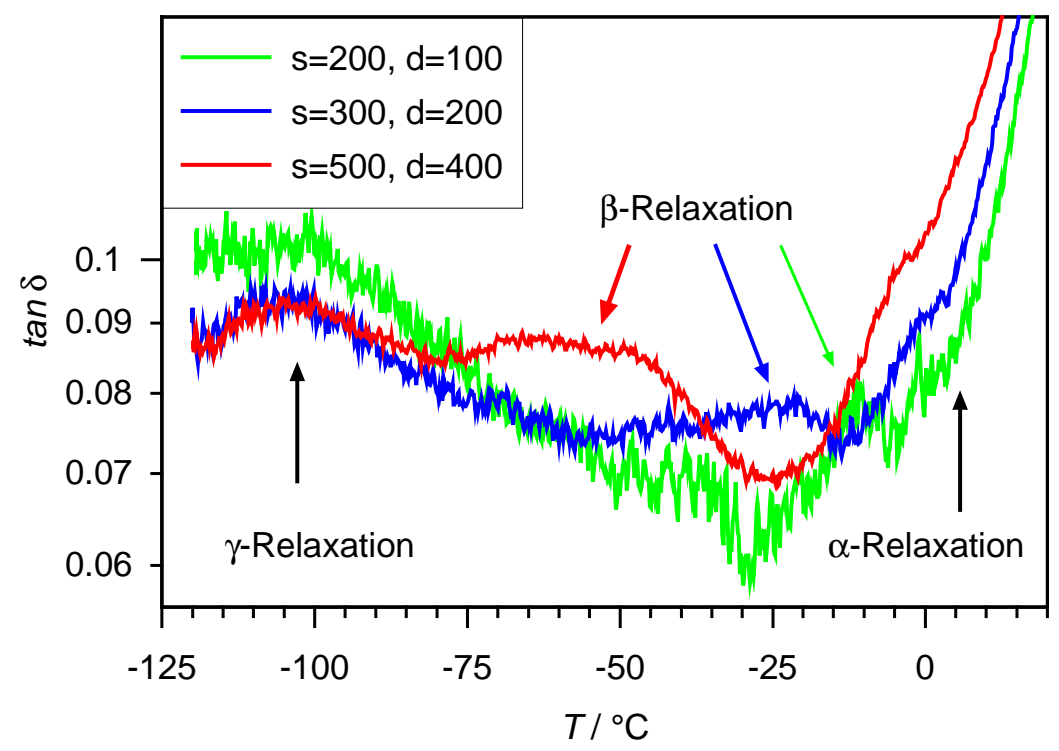

Abbildung 4.41: Verlauf des tan $\delta$ der mechanischen Spektroskopie von unter adiabatischen Bedingungen hergestellten Copolymeren (EMAA 22.14, 1300 bar) mit einem MAA-Gehalt von $F_{M A A}=0.061$ bei $5 \mathrm{~Hz}$, $5{ }^{\circ} \mathrm{C} \cdot \mathrm{min}^{-1}$ und den angegebenen Kräften / $\mathrm{mN}$.

Aus Abbildung 4.41 wird ersichtlich, dass bei der Verwendung von geringeren Kräften sich die Form des $\beta$-Maximums ändert. So führen die Kräfte $s=300 \mathrm{mN}$ und d=200 mN zu einer Abnahme der Intensität des $\beta$-Maximums, wie es erwartet wurde. Sehr interessant ist die Veränderung der Lage des Maximums. Es wird zu höheren Temperaturen verschoben und befindet sich nunmehr bei $-20{ }^{\circ} \mathrm{C}$. Das weitere Absenken der Kräfte auf s=200 mN und $\mathrm{d}=100 \mathrm{mN}$ führt $\mathrm{zu}$ einer weiteren Verschiebung des $\beta$-Peaks in den $\alpha$-Peak. Das $\beta$-Maximum ist nur noch schwach bei $-11^{\circ} \mathrm{C}$ zu erkennen.

Die Analyseergebnisse für höhere Kräfte sind Abbildung $4.42 \mathrm{zu}$ entnehmen. 


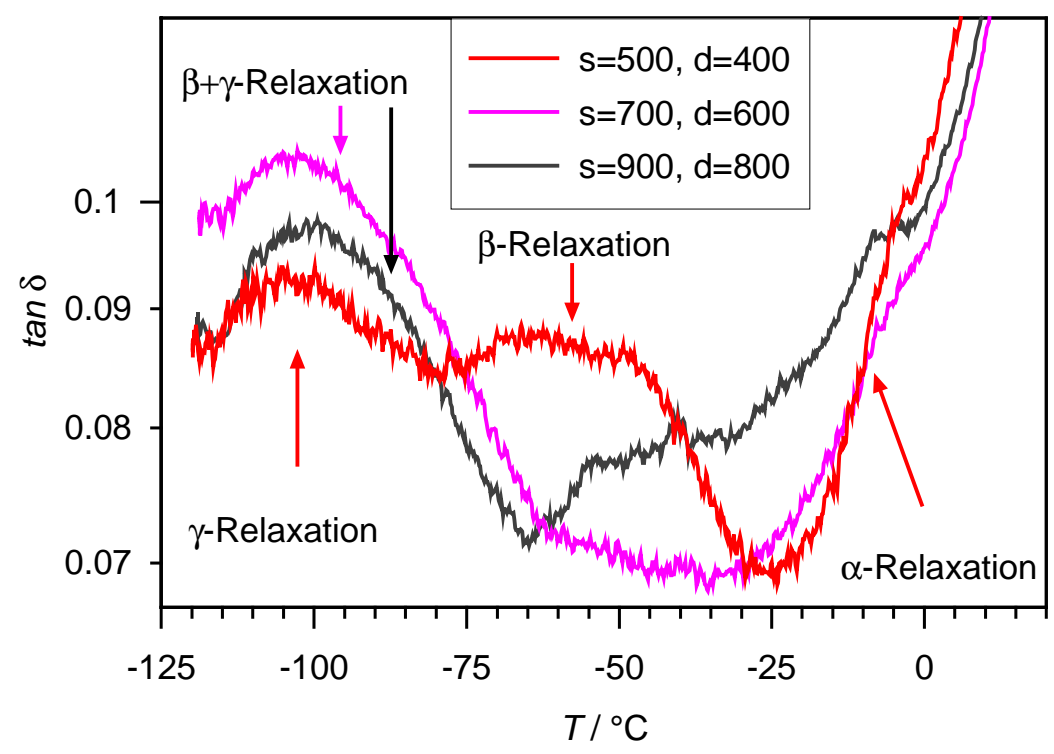

Abbildung 4.42: Verlauf des tan $\delta$ der mechanischen Spektroskopie von unter adiabatischen Bedingungen hergestellten Copolymeren (EMAA 22.14, 1300 bar) mit einem mittlerem MAA-Gehalt von $F_{M A A}=0.061$ bei $5 \mathrm{~Hz}$, $5^{\circ} \mathrm{C} \cdot \mathrm{min}^{-1}$ und den angegebenen Kräften / $\mathrm{mN}$.

Für die verwendeten Kräfte $s=700 \mathrm{mN}$ und $\mathrm{d}=600 \mathrm{mN}$ verschiebt sich das $\beta$-Maximum in den Bereich des $\gamma$-Peaks und wird von diesem überlagert. Die Existenz des $\beta$-Peaks ist an der Schulter, die sich im Bereich von -35 bis $-63{ }^{\circ} \mathrm{C}$ ergibt, zu belegen. Für noch höhere Kräfte von $s=900 \mathrm{mN}$ und $\mathrm{d}=800 \mathrm{mN}$ ist der $\beta$-Peak nicht mehr lokalisierbar. Der $\alpha$-Übergang zeigt hier eine von allen anderen Messungen abweichende Form. Der Peak ist viel breiter und startet bei erheblich tieferen Temperaturen. Der aktuelle Stand der Forschung an Glasbildnern ist die Theorie, dass ein $\alpha$-Übergang aus mehreren Unterschritten besteht. ${ }^{[21,22]}$

Aus der Kräftevariation ist ein Einfluss der angelegten Kräfte auf die Lage des $\beta$-Übergangs festzustellen. In Abbildung 4.43 sind die verwendeten Kräfte gegen die Temperatur des $\beta$-Maximums aufgetragen. Hierzu wurden jeweils die Temperaturen des Maximums der $\beta$-Relaxation verwendet. Für das Kräftepaar $s=700$ und $d=600 \mathrm{mN}$ musste die Temperatur aufgrund der Überlagerung von $\beta$ - und $\gamma$-Relaxation auf $-78{ }^{\circ} \mathrm{C}$ geschätzt werden. Für höhere Kräfte ist eine Auswertung aufgrund der Überlagerung von $\beta$ - und $\gamma$-Relaxation nicht möglich. 


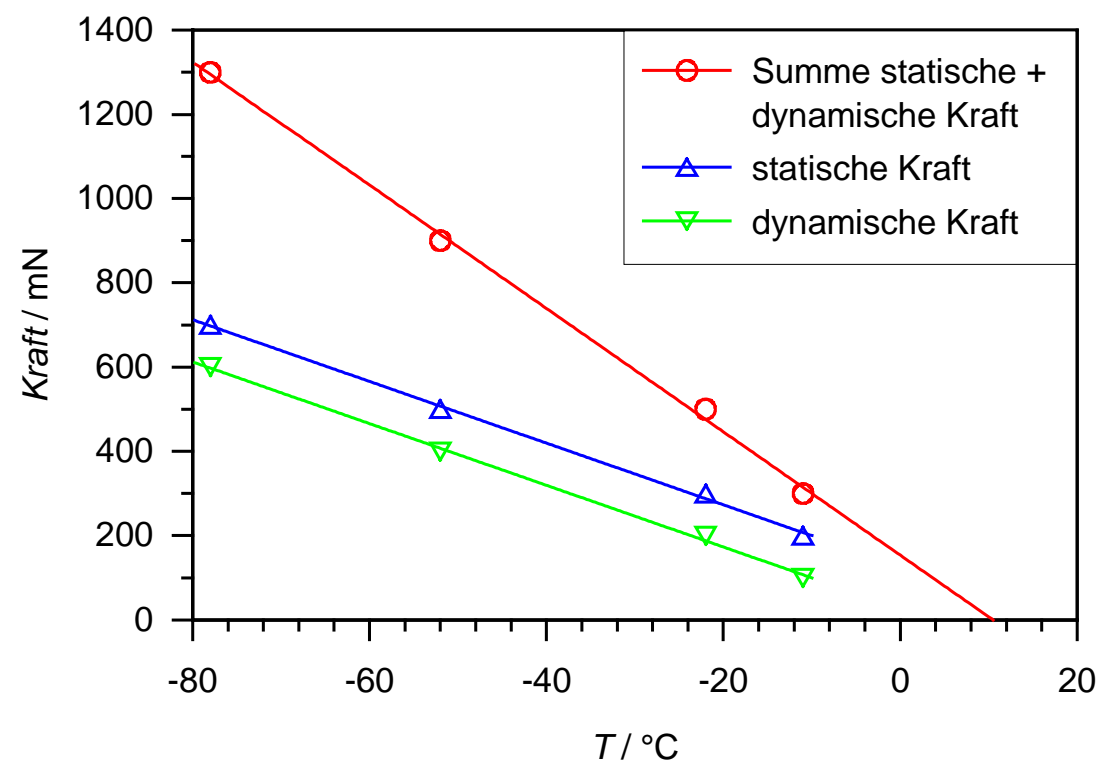

Abbildung 4.43: Einfluss der verwendeten Kräfte auf die Lage des Maximums der beobachteten $\beta$-Relaxation.

In Abbildung 4.43 lässt sich ein linearer Zusammenhang zwischen der Lage des $\beta$-Maximums und den verwendeten Kräften finden. Es zeigt sich, dass die benötigte Energie der $\beta$-Relaxation auch durch eine mechanische Anregung in das Polymer eingebracht werden kann und das die resultierende Lageänderung des $\beta$-Maximums proportional zur verwendeten Kraft ist (siehe auch Kapitel 4.11.4).

\subsubsection{Diskussion}

In diesem Kapitel wurden Dynamisch-mechanische Analysen an EMAA-Copolymeren durchgeführt. Nach Vergleich der resultierenden Spektren mit Daten der beiden Homopolymere und untereinander zeigt sich, dass jeweils die Niederdruckproben über eine zusätzliche Relaxation verfügen, die mit dem $\beta$-Übergang der DSC-Analyse vergleichbar ist, aber nicht bei der gleichen Temperatur auftritt. Unter adiabatischen Bedingungen hergestellte Proben mit einem nahezu gleichen Umsatz zeigten ebenfalls eine $\beta$-Relaxation für die Niederdruckproben, sodass Umsatzeinflüsse ausgeschlossen werden können. Eine Variation der angelegten Kräfte führt zu einer deutlichen Verschiebung des $\beta$-Peaks. Eine Anregung des Copolymers lässt sich in der DMA auf zwei Weisen realisieren. Zum einem besteht die Möglichkeit der thermischen Anregung, äquivalent zu den durchgeführten DSC-Experimenten. Deswegen wurde bei der DMA auch die gleiche Heizrate von 
$5{ }^{\circ} \mathrm{C} \cdot \mathrm{min}^{-1}$ gewählt. Zum anderen kann eine Relaxation durch mechanische Energie hervorgerufen werden. In den DMA-Experimenten setzt sich die Anregung dementsprechend aus den gewählten Temperaturen und Kräften zusammen. Für den bei tiefen Temperaturen als der $\beta$-Übergang beobachteten $\gamma$-Übergang tritt keine Kräfteabhängigkeit auf. Hier wird die für eine Kurbelwellenbewegung der Hauptkette notwendige Energie durch die Temperatur bereitgestellt. Betrachtet wird nun die $\beta$-Relaxation. Hier liegt offensichtlich auch eine mechanische Anregung vor. Durch die Erhöhung der auf die Proben wirkenden Kräfte wird mehr Energie in diese Relaxation eingebracht. Die benötigte thermische Energie ist somit geringer. Resultierend verschiebt sich der $\beta$-Übergang zu tieferen Temperaturen, bis er schließlich komplett von dem $\gamma$-Übergang überlagert wird. Dieses nicht Newtonsche Verhalten wurde ebenfalls bei anderen Glasbildnern beobachtet. ${ }^{[23,24,25]}$

Das Zusammenwirken von mechanischer und thermischer Energie erklärt auch die unterschiedlichen Temperaturen, bei den der $\beta$-Übergang in den DSC-Analysen und den DMA-Analysen beobachtet wurde. Für die DMA-Analysen liegt der jeweilige $\beta$-Übergang aufgrund eingebrachter mechanischer Energie bei tieferen Temperaturen als bei den DSCAnalysen (siehe auch Kapitel 4.10). Werden die Gesamtkräfte in Abbildung 4.43 (rote Kurve) zu Null extrapoliert, so ergibt sich eine Temperatur für das Maximum der $\beta$-Relaxation von ca. $10^{\circ} \mathrm{C}$. Bei diesen Temperaturen werden auch $\beta$-Minima der DSCMessungen beobachtet (siehe z. B. Abbildung 4.28). Hier bestehen also weitere Parallelen zwischen DMA- und DSC-Messungen.

Zusätzlich zu den beschrieben Messreihen, die in Kompression eines zylindrischen Probenkörpers erhalten wurden, gibt es die Möglichkeit in einem Zugversuch die Relaxationen zu beobachten. Hierzu wurden dünne Fäden in die in 3.2.3.3 beschrieben Zugvorrichtung eingebaut. Die Fasern wurden durch das Ziehen von Fäden aus einer aufgeschmolzenen Probe mittels einer Pinzette erhalten. Die Ergebnisse dieser Messreihen zeigen im Ansatz ebenfalls die in der Kompression gemachten Beobachtungen. Es zeigt sich für die Niederdruckproben ebenfalls die Ausbildung eines $\beta$-Übergangs. Die Übergänge sind allerdings nur schwer von dem vorhandenen Grundrauschen zu unterscheiden, sodass von einer Abbildung der Ergebnisse abgesehen wird. Von einigen Proben war es möglich, aus der Rohsubstanz, die aus dem Abscheidevorgang nach der Polymerisation erhalten wurde, einen Streifen herauszuschneiden. Dieser Streifen 
sollte möglichst frei von Gaseinschlüssen sein und eine gleichmäßige Form aufweisen. Die Untersuchungen bestätigten auch hier tendenziell die Ergebnisse der Kompressionsversuche. Das Vorhandensein einer $\beta$-Relaxation bekräftig hier ein weiteres Mal, dass der zusätzliche Übergang nicht auf Effekte während der Probenvorbereitung zurückzuführen ist. Die völlige Gleichbehandlung von Hoch- und Niederdruckproben während des Probenpressens würde bei den beiden Proben, sollte es überhaupt einen signifikanten Einfluss des Pressens geben, zu den gleichen Effekten führen. Da nur die Niederdruckproben einen $\beta$-Übergang zeigen, kann dieser Effekt nicht während der Vorbereitung in das Material eingetragen worden sein. 


\subsection{Dielektrische Spektroskopie an Copolymeren}

Eine weitere Möglichkeit Spektroskopie an nicht leitenden Polymeren zu betreiben bietet neben der DMA auch die dielektrische Spektroskopie (DES). Die bereits vorliegenden DSC- und DMA-Ergebnisse legen die Vermutung nahe, dass auch bei dieser Spektroskopieart ein Unterschied anhand einer zusätzlichen Relaxation im Material zu finden ist. Es erfolgt wieder der direkte Vergleich einer Hoch- und einer Niederdruckprobe. Es wird eine Probenreihe mit vergleichsweise hohem MAA-Gehalt gewählt, nämlich EMAA 3. Hierbei sollten sich, wie in der DSC beobachtet, die signifikantesten Unterschiede ergeben.

Die Messung der Hochdruckprobe EMAA 3.4 führt nicht zu verwertbaren Ergebnissen. Die Probe weist eine zu hohe Härte auf. Der Verlustmodul ist zu gering um befriedigende Ergebnisse zu erhalten. Bei der entsprechenden Niederdruckprobe war eine Analyse möglich. Da somit der Vergleich einer Hoch- und einer Niederdruckprobe für die Probenreihe EMAA 3 nicht realisierbar war, wurden weitere Messungen an einer Hochund einer Niederdruckprobe der Messreihe EMAA 22 durchgeführt. Die resultierenden Spektren sind in Abbildung 4.46 und Abbildung 4.47 aufgeführt.

Abbildung 4.44 zeigt eine Auftragung des dielektrischen Verlusts gegen die Frequenz des verwendeten elektrischen Wechselfeldes bei verschiedenen Temperaturen. Bei niedrigen Frequenzen befindet sich der $\alpha$-Peak des Glasübergangs. Bei hohen Frequenzen ist ein weiterer Peak zu beobachten. Ob es sich dabei um eine $\beta$ - oder eine $\gamma$-Relaxation handelt, ist nicht eindeutig festzustellen. Bei einer Temperatur von $42{ }^{\circ} \mathrm{C}$ (blaue Kurve S ) deutet sich eine Aufspaltung in möglicherweise zwei Beiträge an. Es kann somit vermutet werden, das dieser Übergang sowohl Beiträge eines $\beta$ - und eines $\gamma$-Übergangs enthält. Hier finden sich also auch Hinweise auf das Vorhandensein einer zusätzlichen Relaxation.

Die Anregung in der dielektrischen Spektroskopie unterscheidet sich von der mechanischen Spektroskopie. Bei der dielektrischen Spektroskopie kommt es zur elektrischen Anregung der polaren Gruppen wohingegen bei der mechanischen Spektroskopie ganze Kettensegmente angeregt werden. Somit können sich diese beiden Spektroskopiearten in ihren Ergebnissen erheblich unterscheiden. Es muss weiterhin berücksichtigt werden, dass bei der DES nur der geringe polare Anteil der Copolymere angeregt werden kann. Relaxationen könnten deutlich schwächer zu detektieren sein. 


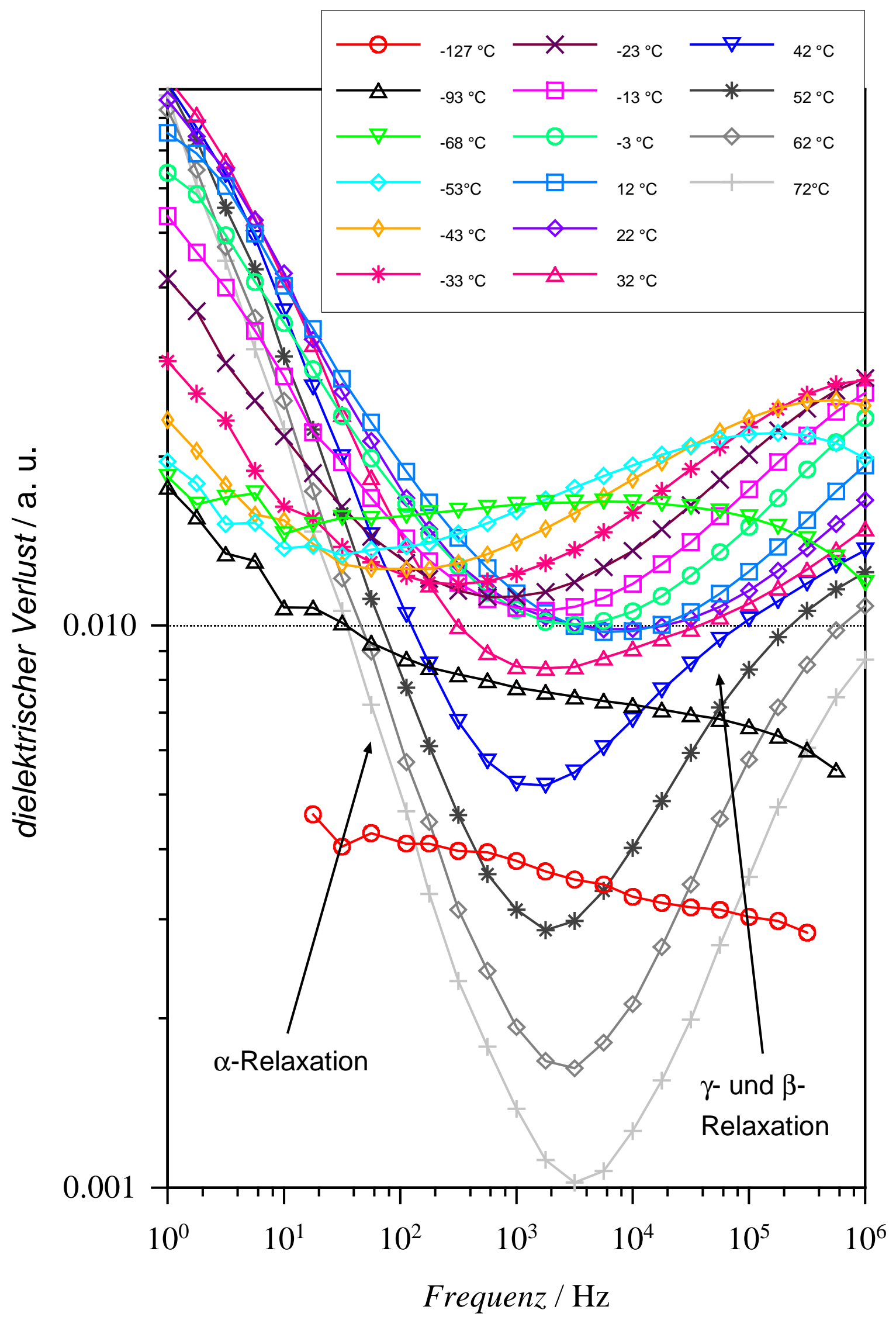

Abbildung 4.44: Dielektrische Spektroskopie an einer Niederdruckprobe (EMAA 3.12, 1300 bar). Gezeigt ist der dielektrische Verlust in Abhängigkeit von der Frequenz bei verschiedenen Temperaturen. 
An der Niederdruckprobe EMAA 22.14 , die bei $240{ }^{\circ} \mathrm{C}$ unter adiabatischen Bedingungen hergestellt wurde, konnten bei der DMA-Spektroskopie starke Effekte und eine Kräfteabhängigkeit der zusätzlichen $\beta$-Relaxation gefunden werden (siehe Kapitel 4.5.4). Nach Kenntnis dieser Effekte werden auch bei der DES die deutlichsten Effekte für die Probe EMAA 22.14 erwartet. Da eine Variation der Temperatur ebenfalls möglich ist, wurde hier die Temperatur in einem Aufheizvorgang variiert und der Verlustmodus in Abhängigkeit der Temperatur anstatt der Frequenz gemessen. Das Ergebnis des Verlustmoduls in Abhängigkeit der Temperatur bei verschiedenen Frequenzen zeigt Abbildung 4.45.

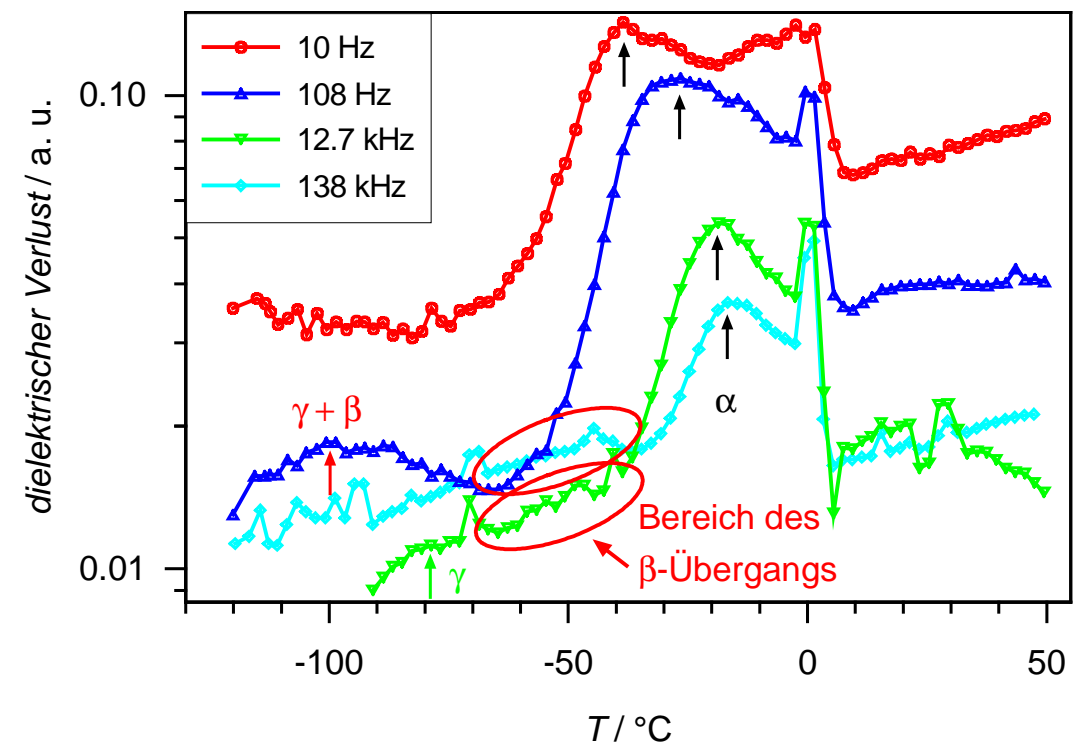

Abbildung 4.45: Dielektrischer Verlust einer Niederdruckprobe (EMAA 22.14, 1300 bar) unter Variation der Temperatur bei verschiedenen Frequenzen.

Die Anomalie, die bei allen gezeigten Kurven bei $0{ }^{\circ} \mathrm{C}$ auftritt, kann auf Wasser in der Probe zurückgeführt werden. Der $\alpha$-Übergang, der durch den schwarzen Pfeil gekennzeichnet ist, ist bei allen verwendeten Frequenzen deutlich zu beobachten. Es zeigt sich eine Frequenzabhängigkeit des $\alpha$-Maximums (schwarzer Pfeil). Das $\alpha$-Maximum ist bei niedrigen Frequenzen $\mathrm{zu}$ niedrigen Temperaturen verschoben. Eine noch stärker Frequenzabhängigkeit wird aufgrund der DMA-Ergebnisse für den $\beta$-Übergang erwartet. Für einen weiteren Peak bei niedrigeren Temperaturen zeigt sich eine erhebliche Frequenzabhängigkeit. Die blaue Kurve $(r$ ), aufgenommen bei einer Frequenz von $108 \mathrm{~Hz}$, zeigt einen gut ausgebildeten Übergang mit einem Maximum bei $-100{ }^{\circ} \mathrm{C}$ (roter Pfeil $\gamma+\beta)$. Die erwartet starke Frequenzabhängigkeit der $\beta$-Relaxation verschiebt den Peak signifikant zu tieferen Temperaturen bei Verwendung niedriger Frequenzen. Bei $10 \mathrm{~Hz}$ 
(i ), zum Beispiel, ist der Übergang bei tiefen Temperaturen von $-120{ }^{\circ} \mathrm{C}$ noch angedeutet. Bei einer Frequenz von 12.7 kHz (s ) kommt es bereits zu einer Überlagerung der zusätzlichen Relaxation mit der $\alpha$-Relaxation. Das Maximum liegt bei einer Temperatur von ca. $-79^{\circ}$ C. Für noch höhere Frequenzen von z. B. $138 \mathrm{kHz}\left(^{-}\right)$ist der zusätzliche Übergang nahezu komplett überlagert und äußert sich nur noch ansatzweise in einem Maximum bei $-44{ }^{\circ} \mathrm{C}$. Die Maxima, die für Frequenzen im kHz-Bereich bei ca. $-70{ }^{\circ} \mathrm{C}$ beobachtet werden, sind zu scharf, um durch eine Relaxation verursacht werden zu können. Hier handelt es sich mit hoher Wahrscheinlichkeit um einen Messfehler. Der gleiche Effekt kann bei $10 \mathrm{~Hz}$ bei einer Temperatur von $-78^{\circ} \mathrm{C}$ beobachtet werden

Die $\gamma$-Relaxation kann bei dieser Probe allerdings auch nicht eindeutig von der $\beta$-Relaxation entkoppelt werden. Es besteht die Möglichkeit einer Überlagerung der beiden Relaxationen. Aufgrund des beobachteten Verlaufs des Verlustmoduls bei Frequenzen von $12.7 \mathrm{kHz}$ und $138 \mathrm{kHz}$ scheint es aber wahrscheinlicher, dass sich die $\gamma$-Relaxation bei tieferen Temperaturen zeigt. Ein möglicher Anteil dieser Relaxation bei einer verwendeten Frequenz von $12.7 \mathrm{kHz}$ ist mit einem grünen Pfeil gekennzeichnet.

Weiteren Aufschluss, ob es sich bei dem gefundenen Übergang bei tiefen Temperaturen um eine Überlagerung von mehreren Prozessen handelt oder ob eine eindeutige Zuordnung $\mathrm{zu}$ einer $\beta$ - oder $\gamma$-Relaxation vorgenommen werden kann, sollte die Analyse einer Hochdruckprobe bringen. Hierzu wurde versucht, ein Spektrum der Hochdruckprobe EMAA 22.9 zu erlangen. Das Ergebnis zeigt Abbildung 4.46.

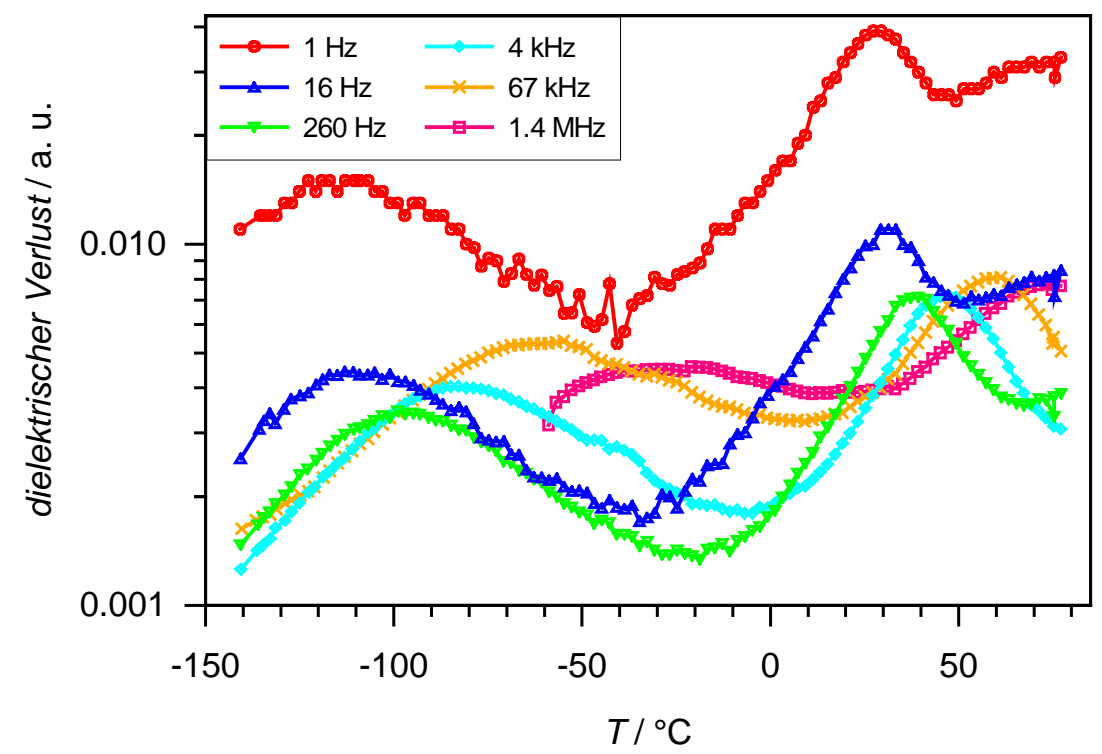

Abbildung 4.46: Dielektrischer Verlust einer Hochdruckprobe (EMAA 22.9, 2300 bar) unter Variation der Temperatur bei verschiedenen Frequenzen. 
Diese Analyse der Probe EMAA 22.9 (2300 bar) sowie die erneute Analyse der Probe EMAA 22.14 (1300 bar) wurden an im Vergleich zur ersten Messung der Probe EMAA 22.14 dünneren Probenkörpern durchgeführt. Um der Problematik eines eventuellen Effektes durch Wasser zu entgehen, wurden die Proben für 3 Stunden ins Vakuum gegeben. Die Messung findet unter Stickstoffatmosphäre statt. Ungereimtheiten im beobachten Kurvenverlauf treten nun bei Temperaturen um $0{ }^{\circ} \mathrm{C}$ nicht mehr auf.

Die $\alpha$-Relaxation der Hochdruckprobe verschiebt, wie bereits in Abbildung 4.45 für die Niederdruckprobe beobachtet, für hohe Frequenzen hin zu höheren Temperaturen. Für alle verwendeten Frequenzen ist die Ausbildung einer weiteren Relaxation bei tieferen Temperaturen $\mathrm{zu}$ beobachten. Diese Relaxation ist deutlich durch die Bildung eines Minimums von der $\alpha$-Relaxation getrennt und weist ebenfalls eine Frequenzabhängigkeit auf.

Um eine direkte Vergleichbarkeit der Messungen für eine Hoch- und eine Niederdruckprobe zu erreichen, wurde eine weitere DES-Messung der Probe EMAA 22.14 durchgeführt.

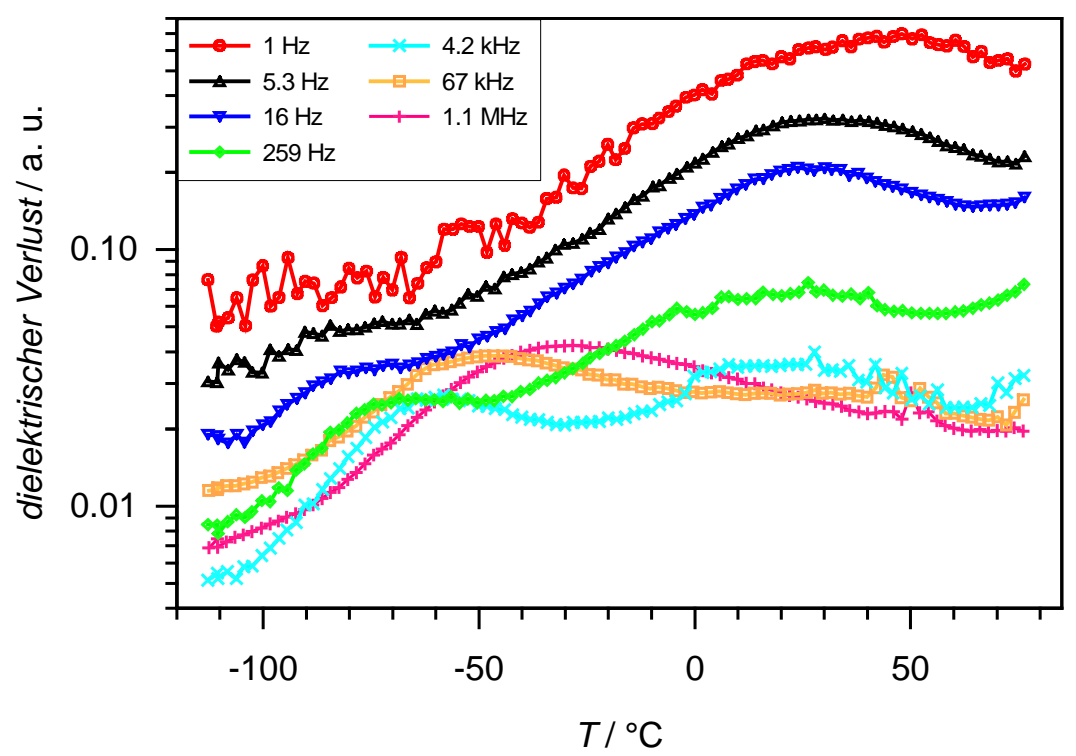

Abbildung 4.47: Dielektrischer Verlust einer Niederdruckprobe (EMAA 22.14) unter Variation der Temperatur bei verschiedenen Frequenzen nach vorangegangener Evakuierung des Probenkörpers.

Der Abbildung 4.47 ist zu entnehmen, dass es im Vergleich zur Hochdruckprobe nicht zu einer klaren Ausbildung eines Minimums kommt. Bei höheren Frequenzen ist die Ausbildung eines lokalen Minimums festzustellen und bei niedrigeren Frequenzen wird 
lediglich eine Schulter beobachtet. Die Relaxation bei tiefen Temperaturen zeigt sich im Vergleich zur Hochdruckprobe eindeutig breiter und undefinierter. Es ist keine Unterscheidung der Relaxation bei tiefen Temperaturen in zwei Anteile, also eine $\beta$ - und eine $\gamma$-Relaxation möglich.

Die Frequenzabhängigkeit der $\alpha$-Relaxation führt nun bei Verwendung von höheren Frequenzen $\mathrm{zu}$ einem Maximum bei niedrigeren Temperaturen, was einem anormalen Verhalten entspricht. ${ }^{[26]}$ Es kommt darüber hinaus teilweise $\mathrm{zu}$ einer erheblichen Verschmelzung des $\alpha$-Peaks mit der bei tieferen Temperaturen einsetzenden Relaxation. Eine $\beta$-Relaxation wird aufgrund der DMA-Messungen im Bereich zwischen der $\gamma$-Relaxation und der $\alpha$-Relaxation erwartet. Das Vorhandensein eines $\beta$-Peaks kann die breite Relaxation bei tiefen Temperaturen erklären und gleichzeitig durch eine Vermischung mit der $\alpha$-Relaxation das entsprechende Maximum zu tieferen Temperaturen ziehen. Dafür spricht auch, dass für die $\beta$-Relaxation ebenfalls ein starke Frequenzabhängigkeit zu erwarten ist. Bei hohen Frequenzen kommt es verstärkt zu einer Verschiebung hin zu hohen Temperaturen und somit zu einer Annäherung an den $\alpha$-Peak. Die daraus resultierende Relaxation weist das Maximum dann entsprechend bei niedrigeren Temperaturen auf im Vergleich zu einer isolierten $\alpha$-Relaxation.

\section{Diskussion:}

Die dielektrische Spektroskopie bekräftigt die mittels DSC und DMA gewonnenen Erkenntnisse. Die Aufnahme des Verlustmoduls für die Niederdruckprobe EMAA 3.12 in Abhängigkeit der Frequenz ergab erste Hinweise auf das Vorhandensein einer zusätzlichen $\beta$-Relaxation. Die Messung einer Hoch- und einer Niederdruckprobe der unter adiabatischen Bedingungen bei $240{ }^{\circ} \mathrm{C}$ hergestellten Probenreihe EMAA 22 zeigt deutliche Unterschiede im dielektrischen Verlust auf. Es ist festzuhalten, dass eine Niederdruckprobe bei niedrigen Temperaturen eine Überlagerung aus mehreren Relaxationen zeigt, was eine Hochdruckprobe nicht aufweist. Diese Überlagerung lässt sich plausibel mittels einer zusätzlichen $\beta$-Relaxation erklären. Überlagerungen der $\alpha$-Relaxation wurden bereits bei Rösner et al beobachtet. ${ }^{[27]}$

Die Hoch- und Niederdruckproben weisen, wie bereits mittels DMA und DSC-Analysen aufgezeigt, Unterschiede auf. Diese lassen sich mittels des „Chemical Confinements“ erklären. Dieses Konzept wird ausführlich in Kapitel 5 beschrieben. 


\subsection{Strukturanalyse mittels Festkörper-NMR-Spektroskopie}

Über die in Kapitel 4.1 beschriebene Gehaltsbestimmung hinaus wurden weitere NMRExperimente durchgeführt. Im Vordergrund stand die Strukturaufklärung der Copolymere. Insbesondere sollen Unterschiede zwischen Hoch- und Niederdruckproben qualitativ und nach Möglichkeit auch quantitativ herausgearbeitet werden. In Kapitel 3.2.8.2 wurde bereits das Auftreten eines zusätzlichen Signals für benachbarte Acrylatgruppen bei einer chemischen Verschiebung von ca. $42 \mathrm{ppm}$ beschrieben, welches aus einer Aufspaltung für eine von Polyethyleneinheiten umgebene Acrylatgruppe entsteht. ${ }^{[3,28,29,30]}$ Diese Aufspaltung wird auch für das Copolymersystem EMAA erwartet.

\subsubsection{Eindimensionale ${ }^{13} \mathrm{C}$-NMR-Spektroskopie an EMAA-Copolymeren}

Bei Messungen an den Probereihen EMAA 2, EMAA 3 und EMAA 12 konnte zunächst keine Aufspaltung festgestellt werden. Bei der unter adiabatischen Bedingungen hergestellten Probenreihe EMAA 16 liegt der höchste ermittelte Säuregehalt im Copolymer vor. Da die Aufspaltung stark von der Anzahl der vorhandenen Säuremoleküle abhängt, besteht für diese Probenreihe die höchste Wahrscheinlichkeit eine Signalaufspaltung zu beobachten. Die Spektren wurden nun nicht mehr bei Raumtemperatur, sondern bei einer Temperatur von ca. $50^{\circ} \mathrm{C}$ aufgenommen. Das führte, wie Abbildung 4.48 zeigt, zu einer erheblichen Verschlechterung der Auflösung. Selbst das Signal für das quartäre C-Atom der Säure wird durch das Grundrauschen weitestgehend überlagert.

Eine Erhöhung der Temperatur während der Detektion führt zu einer Verschlechterung des Signal zu Rausch Verhältnisses. Das beruht wahrscheinlich auf einer höheren Mobilität der Polymerketten bei höherer Temperatur, was sich negativ auf die Transfereffizienz bei der „Cross Polarization“ (CP) auswirkt. Die Übertragung der Polarisierung basiert auf der Dipolkopplung, die von der Orientierung des Dipolvektors zum Magnetfeld abhängt. Durch die bei höheren Temperaturen mögliche Bewegung kann die Dipolkopplung effektiv wegfallen. 


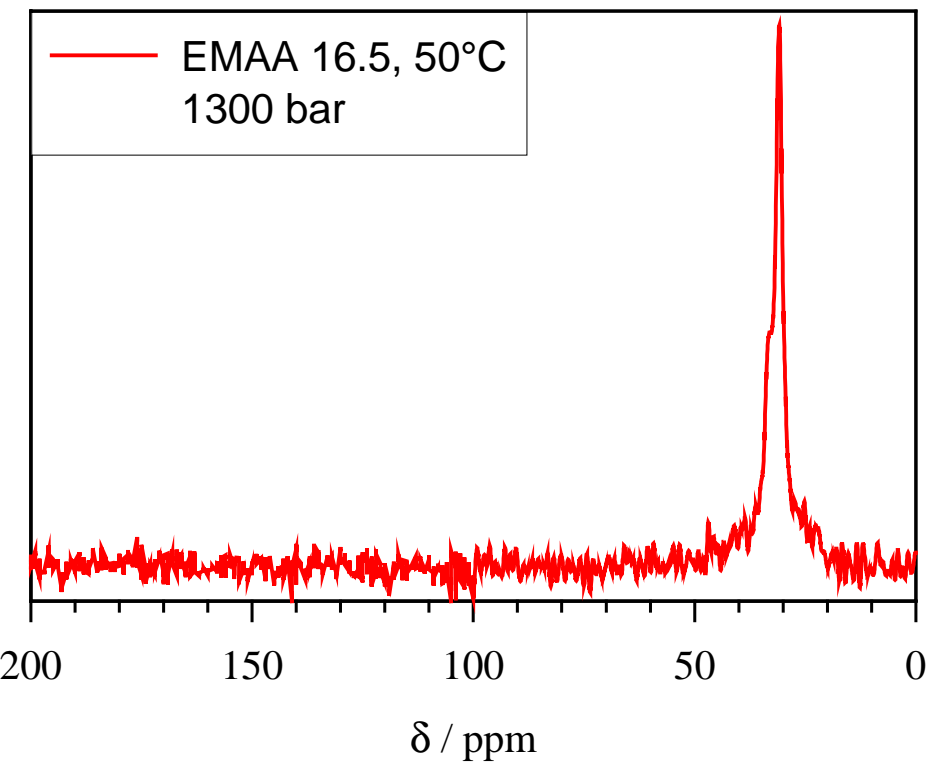

Abbildung 4.48: ${ }^{13} \mathrm{C}$-CP/MAS-NMR-Spektrum von einem unter adiabatischen Bedingungen bei $260{ }^{\circ} \mathrm{C}$ und 1300 bar hergestelltem Copolymer (EMAA 16.5) bei einer Analysentemperatur von $50^{\circ} \mathrm{C}$.

Aus diesem Grund wurde ein weiteres Spektrum bei tieferen Temperaturen aufgenommen. Unter diesen Messbedingungen konnte ein zusätzliches Signal im Bereich von 40 bis $44 \mathrm{ppm}$ gefunden werden. Exemplarisch ist das Spektrum für EMAA 16.5, eine bei 1300 bar hergestellte Niederdruckprobe, in Abbildung 4.49 gezeigt.

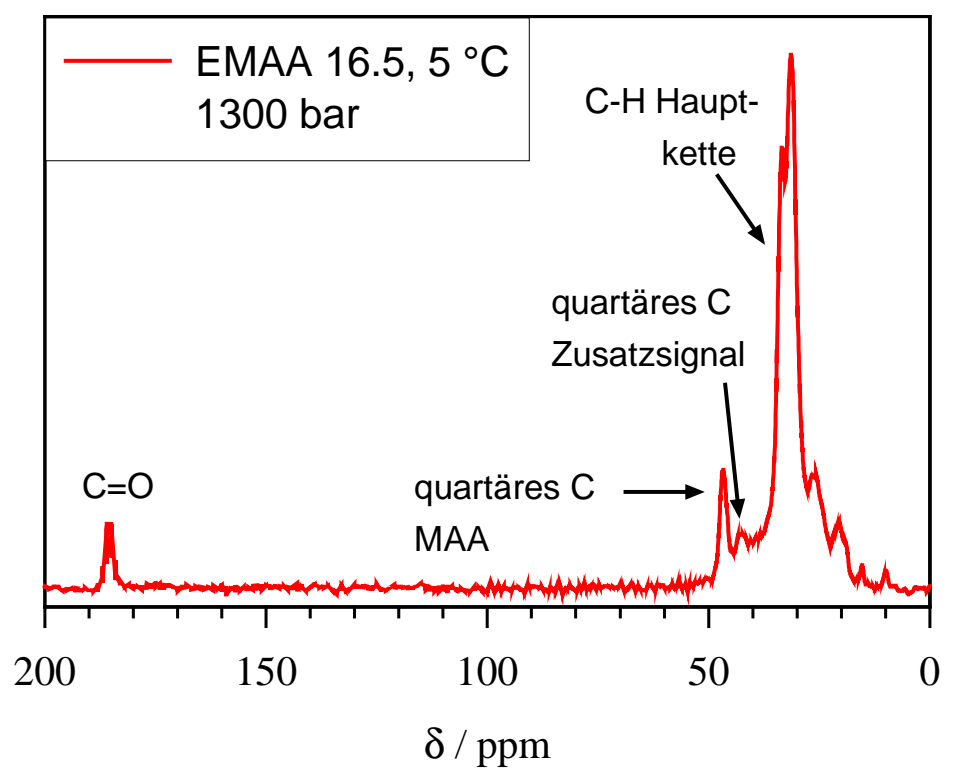

Abbildung 4.49: ${ }^{13} \mathrm{C}$-CP/MAS-NMR-Spektrum von einem unter adiabatischen Bedingungen bei $260^{\circ} \mathrm{C}$ und 1300 bar hergestelltem Copolymer (EMAA 16.5) bei einer Analysentemperatur von $5^{\circ} \mathrm{C}$. 
Ein Signal im Bereich der chemischen Verschiebung von 40 bis 44 ppm ist deutlich zu erkennen. Die Integration ist hier durch die nicht vorhandene Grundlinientrennung der Signale stark fehlerbehaftet. Unter Umständen sind Werte, die aus der Signalhöhe ermittelt werden, vorzuziehen.

Die Integrale der Bereiche 40 bis 44 ppm und 44 bis 50 ppm wurden ins Verhältnis gesetzt, ebenso die entsprechenden Signalhöhen (Abbildung 4.50).

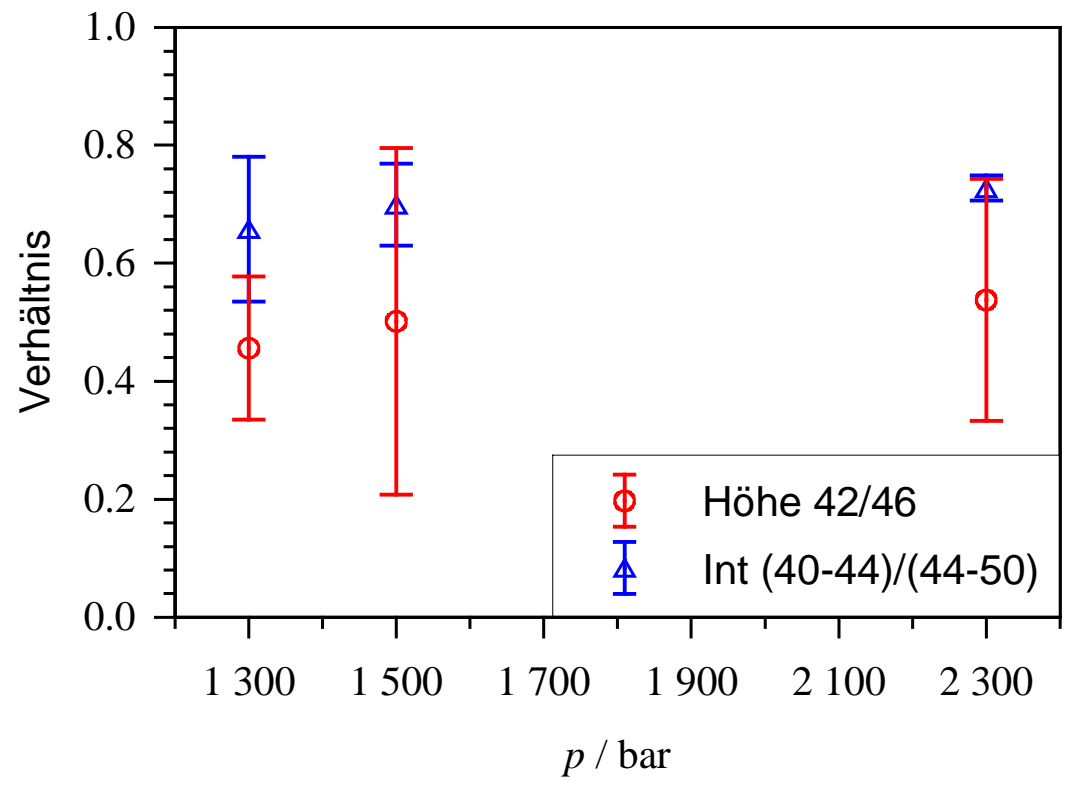

Abbildung 4.50: Verhältnisse der NMR-Signale bei 42 und 46 ppm (siehe Abbildung 4.49) der Probenreihe EMAA 16. Höhe steht für das Verhältnis aus Signalhöhe des Peaks bei 42 ppm dividiert durch die Signalhöhe des Peaks bei 46 ppm. Int steht für das Verhältnis des Integrals von 40 bis 44 ppm dividiert durch das Integral von 44 bis 50 ppm.

Die eingezeichneten Fehlerbalken geben einen Eindruck über die Ungenauigkeit der Verhältnisbestimmung. Die Integralbestimmung erweist sich weniger fehlerbehaftet als der Vergleich der Signalhöhen und wird im Folgenden ausschließlich benutzt. Die Bezeichnung 42/46 steht zukünftig für das Verhältnis des Integrals von 40 bis 44 ppm dividiert durch das Integral von 44 bis 50 ppm.

Die abgebildete Tendenz zeigt einen höheren Wert für 42/46 bei hohen Synthesedrücken. Würde nun das Signal bei $42 \mathrm{ppm}$ benachbarten (direkt nebeneinander in das Copolymer eingebaute) Säuregruppen zugeordnet, so widerspräche das der aufgestellten Theorie, dass Niederdruckproben einen höheren Anteil an zusammenhängenden Säuregruppen haben als die entsprechenden Hochdruckproben. Unter Ausnutzung der Fehlergrenze wäre auch das Gegenteil möglich. Um tiefergehende Aufklärung $\mathrm{zu}$ betreiben, wurden weitere 
Messungen an Copolymeren durchgeführt, die bei gleichem Säuredosierstrom, aber bei verschiedenen Synthesetemperaturen hergestellt wurden.

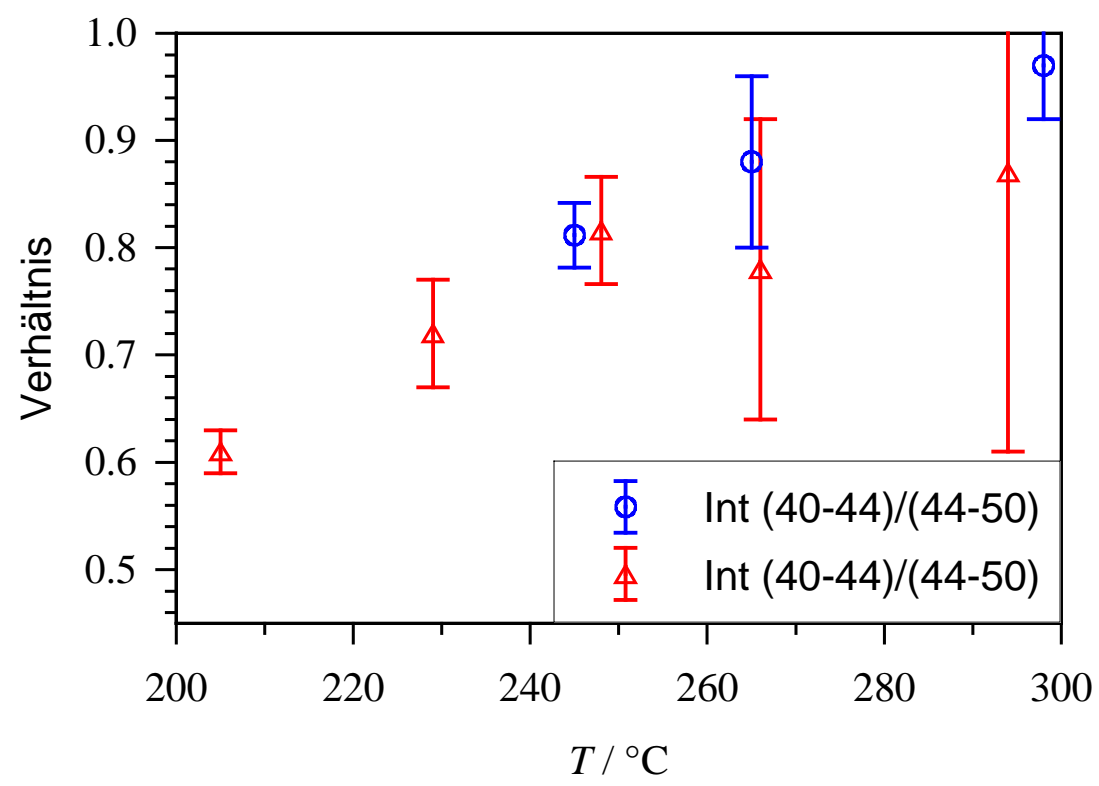

Abbildung 4.51: Integralverhältnis 42/46 für bei verschiedenen Temperaturen synthetisierte Copolymere (EMAA 25 bis 31). Die Proben, die bei 205 und $230{ }^{\circ} \mathrm{C}$ (EMAA 31 und 30) wurden nahe der Phasengrenze bei 2300 bar synthetisiert und zählen somit formal zu den Niederdruckproben (siehe Kapitel 3.1.7.3).

In Abbildung 4.51 liegen erneut die bestimmten Werte für die Niederdruckproben unter denen der entsprechenden Hochdruckprobe. Lediglich die Werte der beiden Proben, die im Temperaturbereich von 245 bis $250{ }^{\circ} \mathrm{C}$ synthetisiert wurden, weisen einen nahezu gleichgroßen Wert auf. Zwar würden hier wiederum die großen Fehlerbereiche auch einen entgegengesetzten Trend dulden, aber es erscheint recht unwahrscheinlich, dass die Niederdruckproben höhere Werte für 42/46 aufweisen, als die Hochdruckproben. Es zeigt sich eine erhebliche Temperaturabhängigkeit für 42/46 für die untersuchten Proben. Bei hohen Synthesetemperaturen liegt ein hoher Wert für das Verhältnis vor. Aufgrund der starken Temperaturabhängigkeit und der geringen Druckabhängigkeit des Wertes sollten Verzweigungen im Copolymer, die durch Transferreaktionen entstehen können, mit in die Diskussion einbezogen werden. ${ }^{[4,19]}$

Wenn es sich bei dem Signal bei 42 ppm um ein quartäres C-Atom handelt, das durch Transferreaktionen entstanden ist, so muss auch das Homopolymer PE dieses Signal zeigen, wenn es bei ausreichend hoher Temperatur synthetisiert wurde. Im Umkehrschluss 
darf das Signal nicht im PE vorhanden sein, wenn es sich um ein Aufspaltungssignal der Methacrylsäureeinheiten handelt.

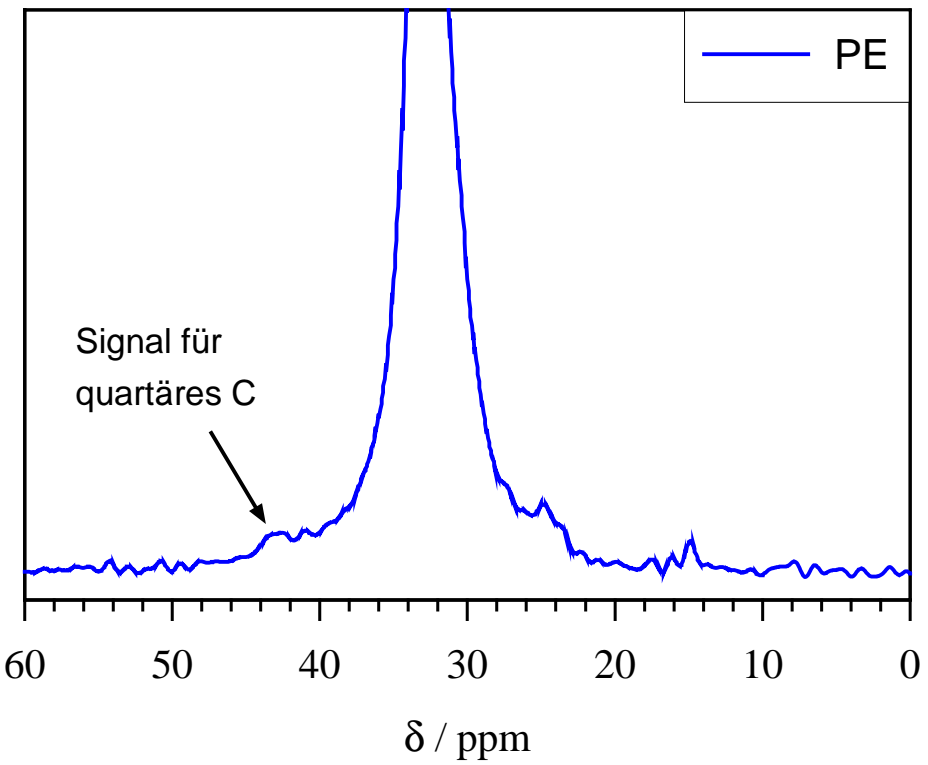

Abbildung 4.52: ${ }^{13} \mathrm{C}$-CP/MAS-NMR-Spektrum eines bei $245{ }^{\circ} \mathrm{C}$ synthetisiertem PE.

Dieses Signal wurde auch in Abbildung 4.52 bei $42.5 \mathrm{ppm}$ für PE gefunden. Die Lage des Signals entspricht vollständig dem beobachteten Peak bei den Copolymeren EMAA. Es kann nun ausgeschlossen werden, dass es sich bei dem Signal um ein für die Säurecopolymere spezifisches Signal handelt. Eine Aufspaltung des Signals für das quartäre C-Atom der Säure sollte zu einem Signal führen, das hochfeldiger liegt als das Signal bei 46 ppm. Diese Aufspaltung kann aber nicht beobachtet werden, oder wird von anderen Signalen überlagert. Eine Strukturaufklärung, die Hoch- und Niederdruckproben unterscheiden kann, ist mit eindimensionalen Experimenten mittels Festkörper-NMRSpektroskopie mit den aufgezeigten Verfahren nicht möglich. Zweidimensionale NMRSpektroskopie kann weitere Aufschlüsse geben.

Die aufgenommenen Spektren erlauben eine Auswertung des Signals bei 42.5 ppm, das hauptsächlich durch Verzweigungen verursacht wird, im Verhältnis zur Hauptkette des Polymers. Erwartungsgemäß sind hier die Fehlerintervalle ebenfalls hoch, wie Abbildung 4.53 belegt. 


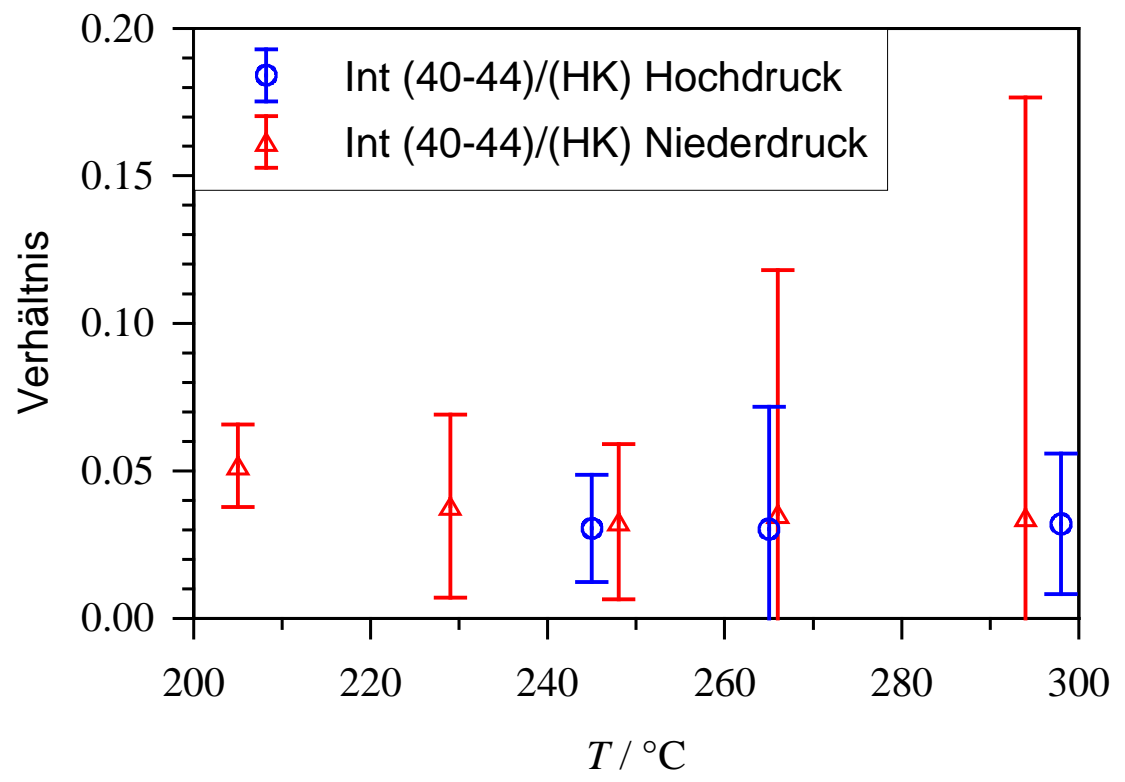

Abbildung 4.53: Verhältnis des Integrals (Int) im Bereich von 40 bis 44 ppm dividiert durch das Integral des Signals der Hauptkette (HK), das aus den Teilintegralen von 22 bis 40 ppm und 44 bis 50 ppm besteht (siehe Abbildung 4.49) für die Copolymere der Probenreihe EMAA 25-31.

In Kapitel 3.1.7.3 wurde bereits die Problematik der Probenherstellung bei Synthesetemperaturen unterhalb von $240{ }^{\circ} \mathrm{C}$ ausführlich erläutert. Misst man nun dem Messwert bei $205^{\circ} \mathrm{C}$ dementsprechend wenig Bedeutung bei, da die Synthesebedingungen hier nicht einfach und eindeutig waren, so zeigen die verbleibenden Werte einen sehr ähnlichen Anteil an Verzweigungen auf. Die Hoch- und Niederdruckproben unterscheiden sich sehr geringfügig, wobei die Werte für Niederdruckproben stets höher liegen als die Werte für Hochdruckproben. Bei der Verwendung von Ausgleichsgeraden ergeben sich für die Probenreihen, die bei mehr als $240{ }^{\circ} \mathrm{C}$ synthetisiert wurden, leicht positive Steigungen. Aufgrund der Fehler, die mittels des Grundrauschens berechnet werden können, ist es allerdings nicht sinnvoll hier von einem wirklichen Trend auszugehen.

\subsubsection{Zweidimensionale NMR-Spektroskopie an EMAA-Copolymeren zur speziellen Strukturaufklärung der Niederdruckproben}

Die aufgezeigten eindimensionalen Experimente konnten nicht zur Strukturunterscheidung von Hoch- und Niederdruckproben herangezogen werden. Zweidimensionale NMRExperimente könnten durch die Aufteilung des Detektorsignals in zwei Dimensionen zu einer Separation von Signalen führen, die sich bei eindimensioneller Detektion überlagern. 
Mit der heteronuklearen Korrelationsspektroskopie, abgekürzt HETCOR, können Kopplungen zwischen Protonen und ${ }^{13} \mathrm{C}$-Kernen beobachtet werden. Der Arbeitsansatz besteht darin, dass unterschiedliche Signale für konjugierte, also in direkter Nachbarschaft in das Copolymer eingebaute, und isolierte Säuregruppen erwartet werden. Das Kopplungsmuster, bei dem die quartären ${ }^{13}$ C-Atome der Säuregruppen beteiligt sind, sollte, je nach Nachbarschaft, unterschiedlich sein.

Abbildung 4.54 zeigt das Hetcor-Spektrum einer Hochdruckprobe. Es ergibt sich ein schwaches Kreuzsignal bei $46 \mathrm{ppm}\left({ }^{13} \mathrm{C}\right)$ und $1.5 \mathrm{ppm}\left({ }^{1} \mathrm{H}\right)$. Dieses Signal wird durch eine Kopplung der quartären ${ }^{13} \mathrm{C}$-Atome mit einer Methylgruppe verursacht. Dass es sich bei dem Kopplungspartner um die Protonen der Methylgruppe handelt, kann anhand eines weiteren Kreuzsignal belegt werden. Das C-Atom der Methylgruppe besitzt eine chemische Verschiebung von ungefähr 15 ppm (siehe Kapitel 4.1). Es sollte sich eine starke Kopplung zwischen diesem C-Atom und den Protonen der Methylgruppe ergeben. Diese Kopplung liegt im Wesentlichen bei einer chemischen Verschiebung der Protonen von 0 bis 2 ppm. Das ist der gleiche Bereich für die Protonenverschiebung wie er auch bei der Kopplung mit dem quartären C-Atom bei 46 ppm beobachtet wird.

Das Hauptsignal im Bereich von 30 ppm beruht auf C-H-Kopplungen der Hauptkette des Polymers. Hieraus lassen sich nur schwer Informationen erhalten, da die Signale sich gegenseitig überlagern.

Abbildung 4.54 zeigt ein Übersichtsspektrum einer Hochduckprobe EMAA 2.2. Eventuelle Unterschiede zwischen einer Hoch- und der entsprechenden Niederdruckprobe sollten bei Detailansichten besser zu erfassen sein. Aus diesem Grund werden in Abbildung 4.55 und Abbildung 4.56 Detailausschnitte für die Hochdruckprobe EMAA 2.2 und die Niederdruckprobe EMAA 2.10 gezeigt. 


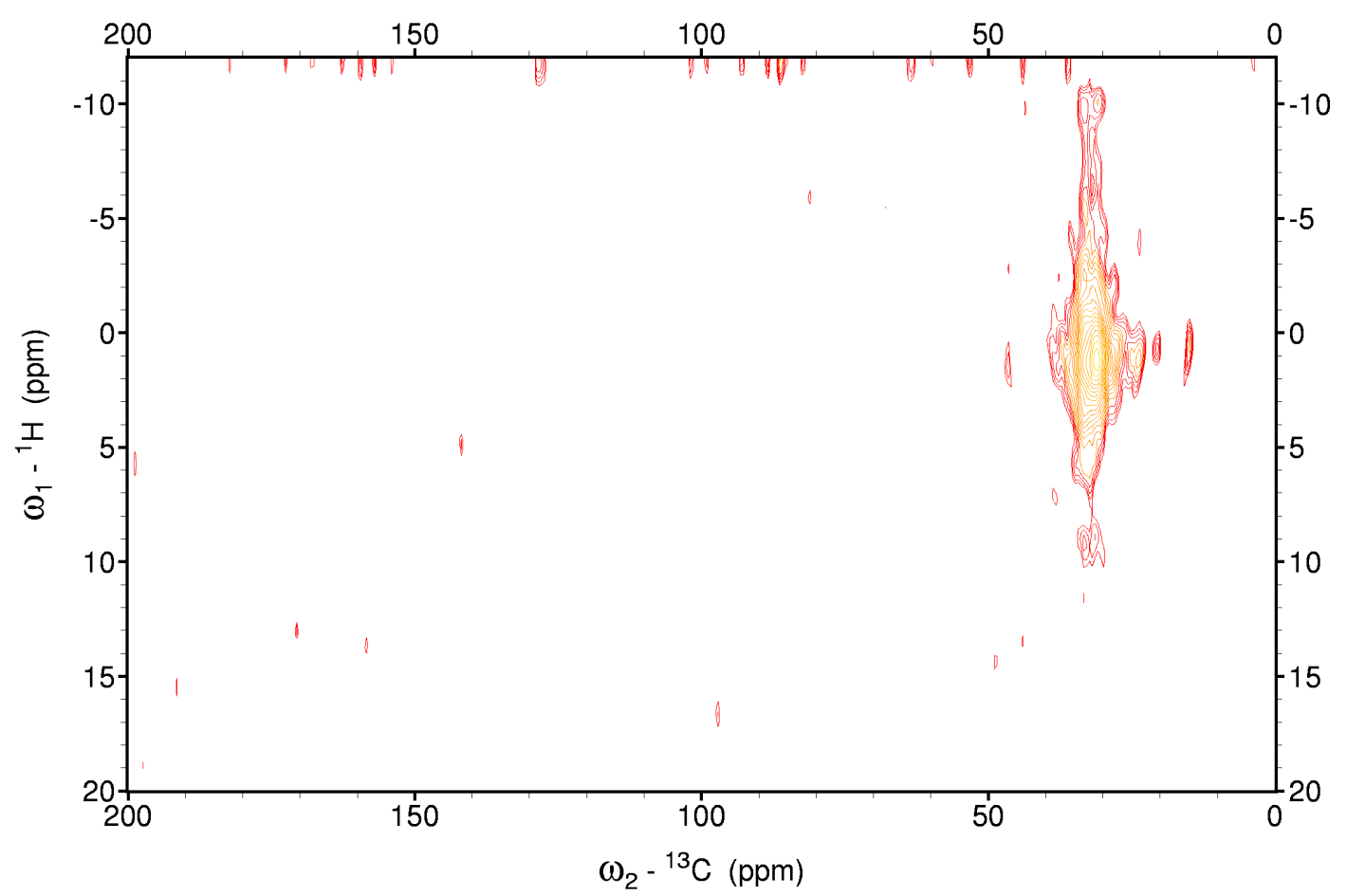

Abbildung 4.54: 2D ${ }^{1} \mathrm{H}_{-}{ }^{13} \mathrm{C}$ HETCOR-NMR-(Übersichts-)Spektrum der Hochdruckprobe EMAA 2.2, aufgenommen bei $400 \mathrm{MHz}$ Protonen-Larmorfrequenz.

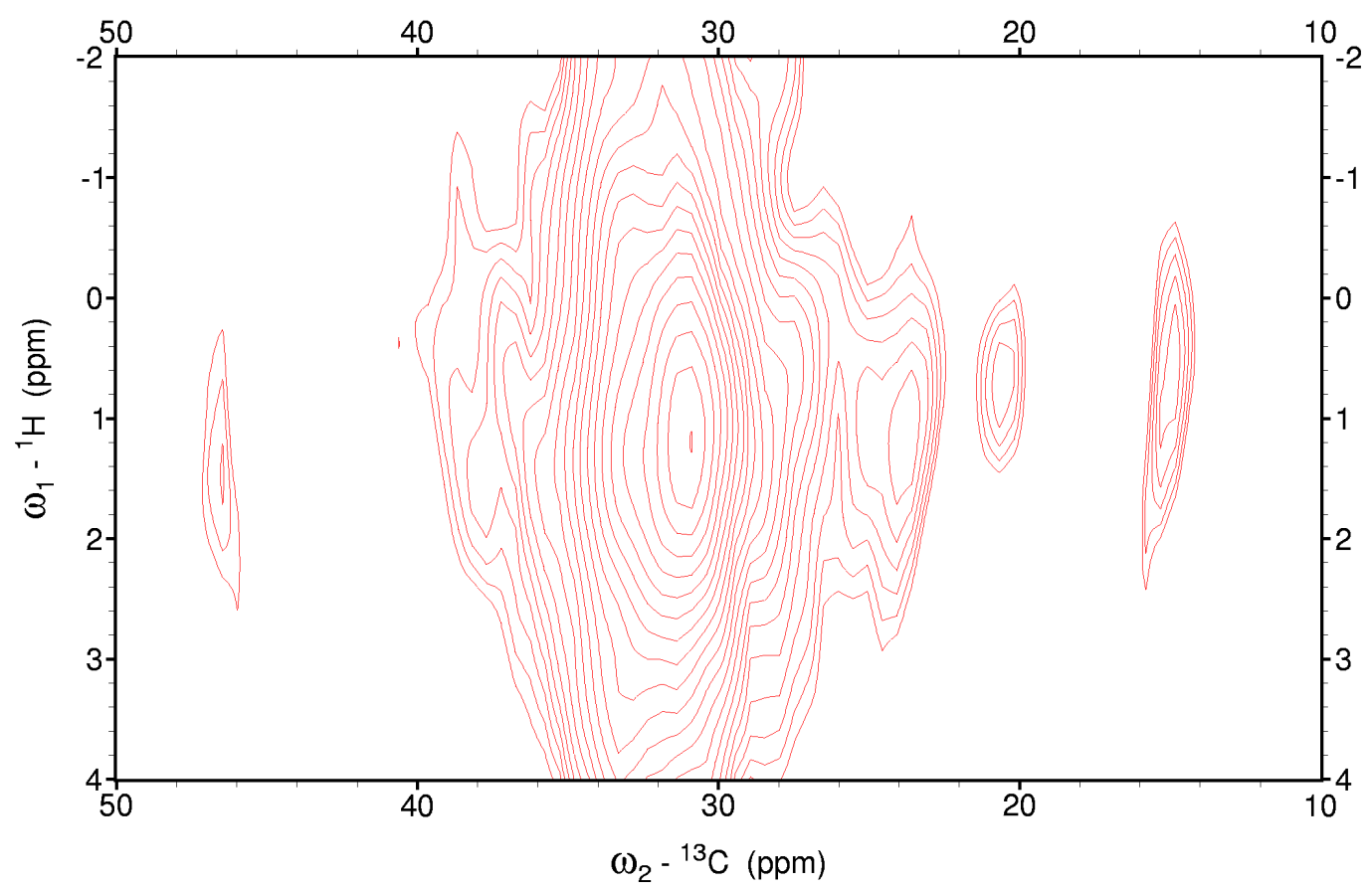

Abbildung 4.55: 2D ${ }^{1} \mathrm{H}_{-}{ }^{13} \mathrm{C}$ HETCOR-NMR-Spektrum der Hochdruckprobe EMAA 2.2, aufgenommen bei $400 \mathrm{MHz}$ Protonen-Larmorfrequenz. 
Der direkte Vergleich des Spektrums einer Hochdruckprobe mit dem entsprechenden Niederdruckprobenspektrum sollte Unterschiede speziell im Bereich von $46 \mathrm{ppm}$ für das quartäre C-Atom zeigen.

In Abbildung 4.56 lassen sich geringe Unterschiede zu Abbildung 4.55 feststellen. Das Kreuzsignal bei 46 ppm $\left({ }^{13} \mathrm{C}\right)$ und 1 ppm $\left({ }^{1} \mathrm{H}\right)$ hat an Signalintensität gewonnen. Das lässt sich anhand der Integrale belegen. Das Verhältnis des Kreuzsignals bei 46 und 1 ppm zum Hauptpeak bei 31 und 1 ppm beträgt für die Probe EMAA 2.2 3.8.10 ${ }^{-2}$ und für EMAA 2.10 $6.3 \cdot 10^{-2}$. Das Verhältnis der beiden Integrale ist für die Niederdruckprobe ca. um den Faktor 1.7 größer als für die Hochdruckprobe.

Eine zusätzliche Kopplung kann aber auch hier nicht beobachtet werden. Die Intensitätszunahme könnte anhand von benachbarten Säuregruppen erklärt werden. Wenn sich Säuregruppen in unmittelbarer Umgebung einer anderen Säuregruppe befinden, so nimmt die Anzahl der Methylgruppen dementsprechend zu. Eine zusätzliche Kopplung zu diesen Methylprotonen ist möglich. Die Kopplung erstreckt sich dann allerdings über vier Bindungen anstatt über zwei. Diese Kopplung fällt relativ schwach aus, kann aber den Intensitätsanstieg des Kreuzsignals plausibel erklären.

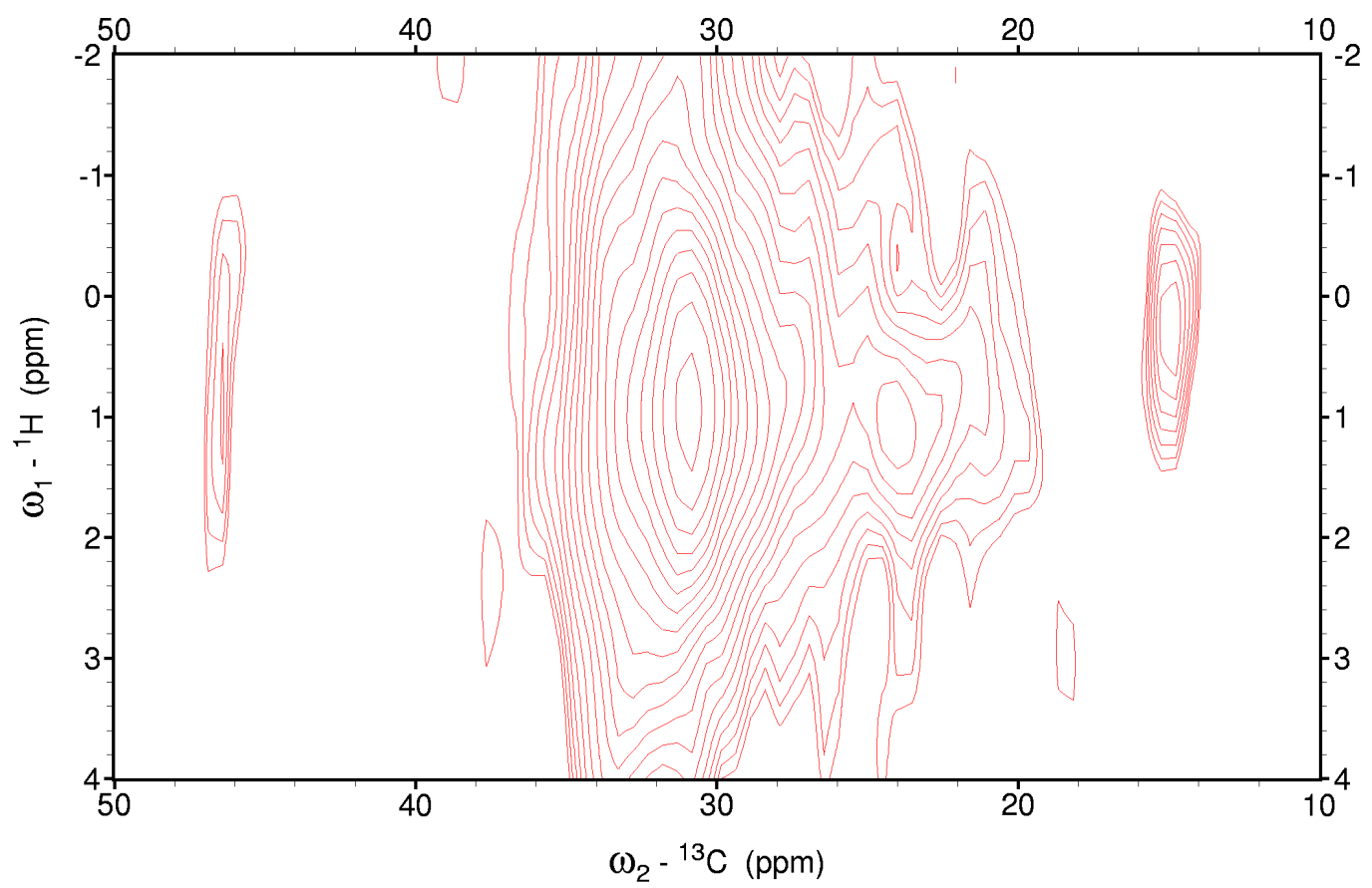

Abbildung 4.56: $2 \mathrm{D}^{1} \mathrm{H}_{-}{ }^{13} \mathrm{C}$ HETCOR-NMR-Spektrum der Niederdruckprobe EMAA 2.10, aufgenommen bei $400 \mathrm{MHz}$ Protonen-Larmorfrequenz. 
Das Problem dieser HETCOR-Spektroskopie liegt in der Häufigkeit des zu untersuchenden Kerns. Das detektierbare ${ }^{13} \mathrm{C}$ besitzt eine relative Häufigkeit von ca. $1 \%$. Die Copolymere besitzen einen Säuregehalt, der in allen Fällen kleiner als 10 mol-\% ist. Somit ist die Häufigkeit der detektierbaren Kerne sehr gering. Die Anzahl an benachbarten Säuregruppen ist ebenfalls ziemlich gering. Ein Aufspaltungssignal ist somit sehr schwer zu beobachten. Eine Möglichkeit wäre die Anreicherung der Methacrylsäure mit ${ }^{13} \mathrm{C}$-Atomen. Da nach der Synthese keine ${ }^{13} \mathrm{C}$-Dotierung mehr vorgenommen werden kann, müsste bereits dotiertes Monomer verwendet werden. Aufgrund der erläuterten Versuchsdurchführung und den damit verbundenen Substanzmengen, ist diese Vorgehensweise aus finanzieller Sicht nicht realistisch.

Aus den genannten Gründen wurden im Folgendem Experimente durchgeführt, die auf Protonen-Protonen Kopplung beruhen. Das stärkste Signal entsteht wiederum durch die Kopplungen der Protonen der Hauptkette. Von besonderer Bedeutung sind die Kreuzsignale, bei denen $\mathrm{OH}-$ Protonen der Methacrylsäure beteiligt sind. Zur Verdeutlichung wurden diese Signale um den Faktor 200 in der Abbildung 4.57 verstärkt. 


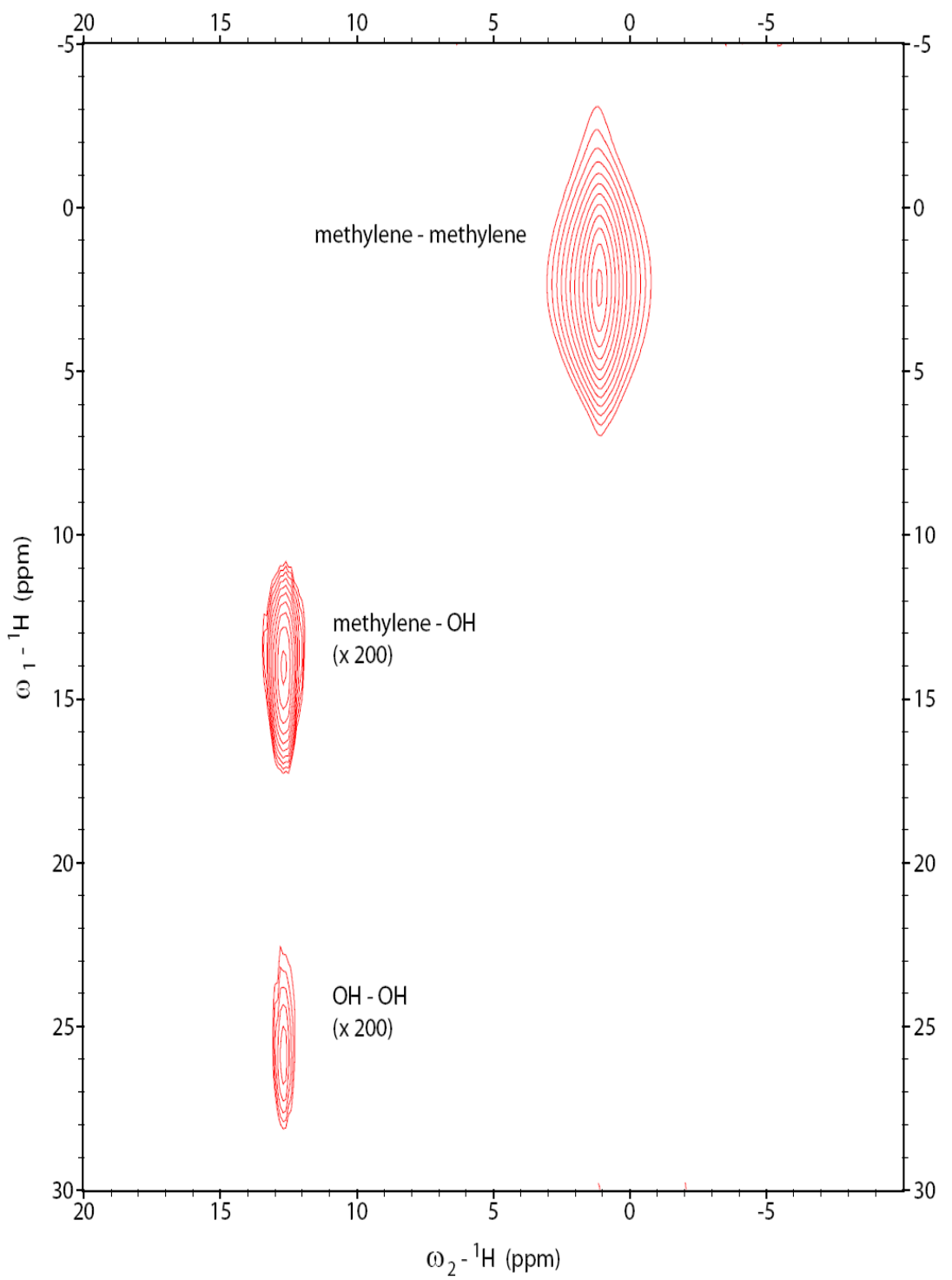

Abbildung 4.57: 2D ${ }^{1} \mathrm{H}_{-}{ }^{1} \mathrm{H}$ Korrelationsspektrum der Niederdruckprobe EMAA 2.10, aufgenommen bei $800 \mathrm{MHz}$ Protonen-Resonanzfrequenz. $\omega_{1}$ entspricht der Resonanzfrequenz in der ersten und $\omega_{2}$ in der zweiten Dimension. OH-Signale wurden 200 mal verstärkt abgebildet.

Es wird eine deutliche Separation der beiden $\mathrm{OH}-$ Kreuzsignale beobachtet. Die Wechselwirkung der OH-Protonen mit der Hauptkette resultieren in einem Signal bei $14 \mathrm{ppm}\left(\omega_{1}\right)$ und $13 \mathrm{ppm}\left(\omega_{2}\right)$. Die Wechselwirkung der OH-Protonen untereinander zeigt sich bei $26 \mathrm{ppm}\left(\omega_{1}\right)$ und $13 \mathrm{ppm}\left(\omega_{2}\right)$. Bei Säuregruppen in direkter Nachbarschaft erhöht sich der Anteil der OH-OH-Kopplung in Relation zur OH-Hauptketten (HK)-Kopplung. 


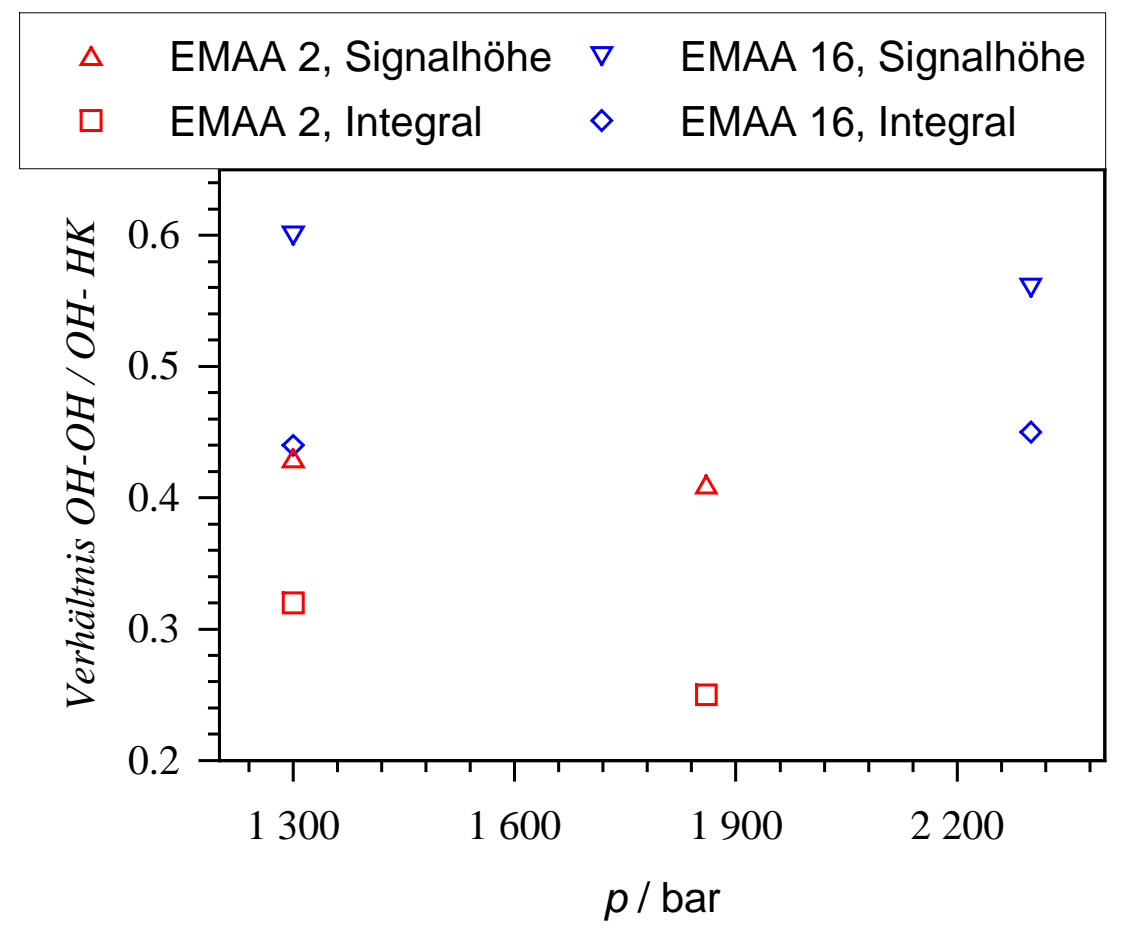

Abbildung 4.58: Verhältnis der Signalhöhe (Dreiecke) der OH-OH Kopplung zur Signalhöhe der OH-Hauptketten (HK)-Kopplung für Hoch- und Niederdruckproben der Probenreihen EMAA 2 (rot) und EMAA 16 (blau) sowie das entsprechende Verhältnis der Integrale (Vierecke).

Es wurden Spektren für die Probenreihen EMAA 2 und EMAA 16 aufgenommen. Das Verhältnis der Signalhöhe der $\mathrm{OH}-\mathrm{OH}-\mathrm{Kopplung}$ zur Signalhöhe der OH-Hauptketten (HK)-Kopplung für die jeweiligen Proben, sowie das entsprechende Verhältnis der Integrale zeigt Abbildung 4.58. Die Auswertung der Verhältnisse der Signalhöhen führt für beide Probenreihen zu einem abnehmenden Anteil an OH-OH-Kopplungen in Relation zur OH-HK-Kopplung bei ansteigendem Synthesedruck. Bei Verwendung des Integralverhältnisses zeigt sich für die Probenreihe EMAA 16 nahezu keine Druckabhängigkeit, wo hingegen die Probenreihe EMAA 2 der gleichen Trend aufzeigt, wie die Auswertung mittels der Signalhöhen.

Das Verhältnis OH-OH/OH-HK ist für die Probenreihe EMAA 16 höher im Vergleich zur Probenreihe EMAA 2. Das kann auf einen erheblich höheren Säureanteil im Copolymer zurückgeführt werden (EMAA 2 ca. 2.5 mol-\%, EMAA 16 ca. 8 mol-\%, siehe Tabelle 4.1).

Ein höherer Anteil der $\mathrm{OH}-\mathrm{OH}-\mathrm{Kopplungen} \mathrm{(im} \mathrm{Vergleich} \mathrm{zu} \mathrm{OH-HK)} \mathrm{für} \mathrm{Niederdruck}$ bestätigt, dass Säuregruppen der Niederdruckproben mehr Säuregruppen in unmittelbarer Nachbarschaft haben, als Hochdruckproben. Die OH-OH-Kopplungen entstehen durch intermolekulare und durch intramolekulare Wechselwirkungen. Bei den intramolekularen 
Kopplungen ist zu berücksichtigen, dass Kopplungen wahrscheinlicher sind, wenn sich die Säuregruppen in der direkten Umgebung einer weiteren Säuregruppe befinden und nicht erst eine günstige Ausrichtung der Polymerkette vorliegen muss. Es kann also gefolgert werden, dass eine Erhöhung der $\mathrm{OH}-\mathrm{OH}-\mathrm{Kopplungen} \mathrm{hauptsächlich} \mathrm{auf} \mathrm{einer} \mathrm{verstärkten}$ Bildung von Dimer- oder Trimersäureeinheiten im Copolymer während der Synthese beruht. 


\subsection{Ergebnisse der gepulsten Laserdeposition}

Die gepulste Laserdeposition (pulsed laser deposition PLD) eignet sich zum Herstellen von dünnen Schichten unterschiedlichster Materialklassen. ${ }^{[31,32,33]}$ Die PLD-Methode findet häufig Anwendung zur Deposition von Metallen. In diesem Abschnitt soll auch auf Depositionen von Polymeren eingegangen werden. Es besteht die Möglichkeit, dass sich die mittels PLD hergestellten Schichten für Hoch- und Niederdruckproben in ihrer Oberflächenstruktur unterscheiden.

\subsubsection{PLD von Metallen auf Silizium-Oberflächen}

Für die PLD von Metallen sind bereits einige Beispiele getestet worden. ${ }^{[34,35]}$ Beispielhaft ist hier eine Rasterelektronenmikroskopaufnahme (REM-Aufnahme) von deponiertem Kupfer auf Silizium gezeigt (Abbildung 4.59) bei einer Probenkammertemperatur von $240{ }^{\circ} \mathrm{C}$.

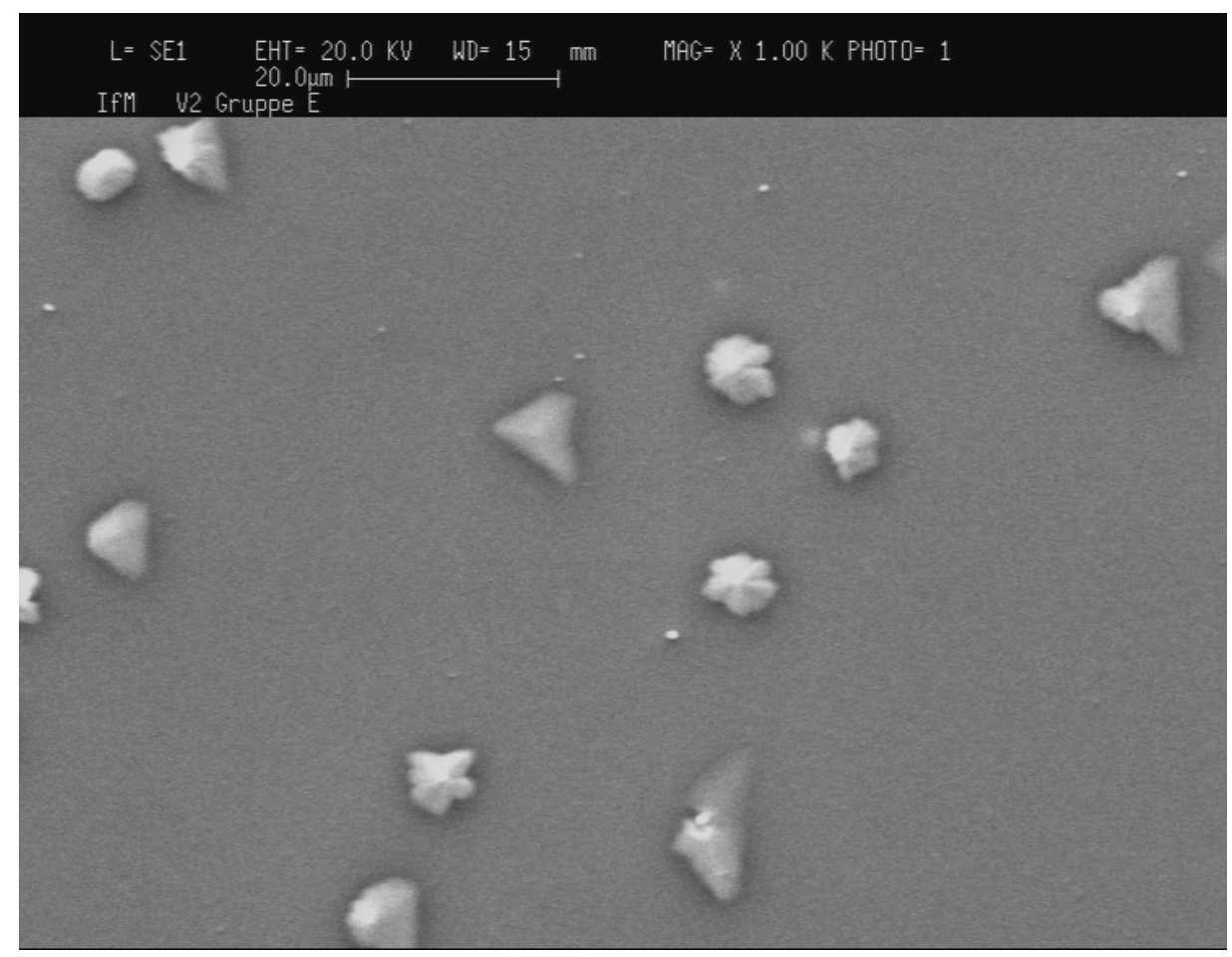

Abbildung 4.59 Rasterelektronenmikroskopaufnahme von auf einer Siliziumoberfläche deponiertem Kupfer. Der Maßstab entspricht $20 \mu \mathrm{m}$ (ca. 1000fache Vergrößerung). 
Die PLD-Methode ist etabliert für Metalle, metallische Legierungen und für Keramiken. Bei Polymeren ist die Anwendung immer noch umstritten. Das Ziel der Forschung ist es in erster Linie dünne Filme entsprechend dem Targetmaterial herzustellen, zum Beispiel unter Beibehaltung der gleichen Molmassenverteilung des Materials. Wünschenswert wäre dann eine Herstellung von homogenen Schichten, wie es für Metalle möglich ist. Da immer noch die Mechanismen der PLD bei Verwendung von Polymeren ungeklärt sind, bedarf es hier weiterer Forschung.

\subsubsection{PLD von aus polaren Monomeren bestehenden Polymeren}

Arbeiten, die sich mit der PLD an Polymeren befassen, sind bereits einige vorhanden. ${ }^{[36,37,38,39,40,41,42,43]}$ Allerdings beziehen sich diese Untersuchungen hauptsächlich auf das Homopolymer Polymethylmethacrylat (PMMA).

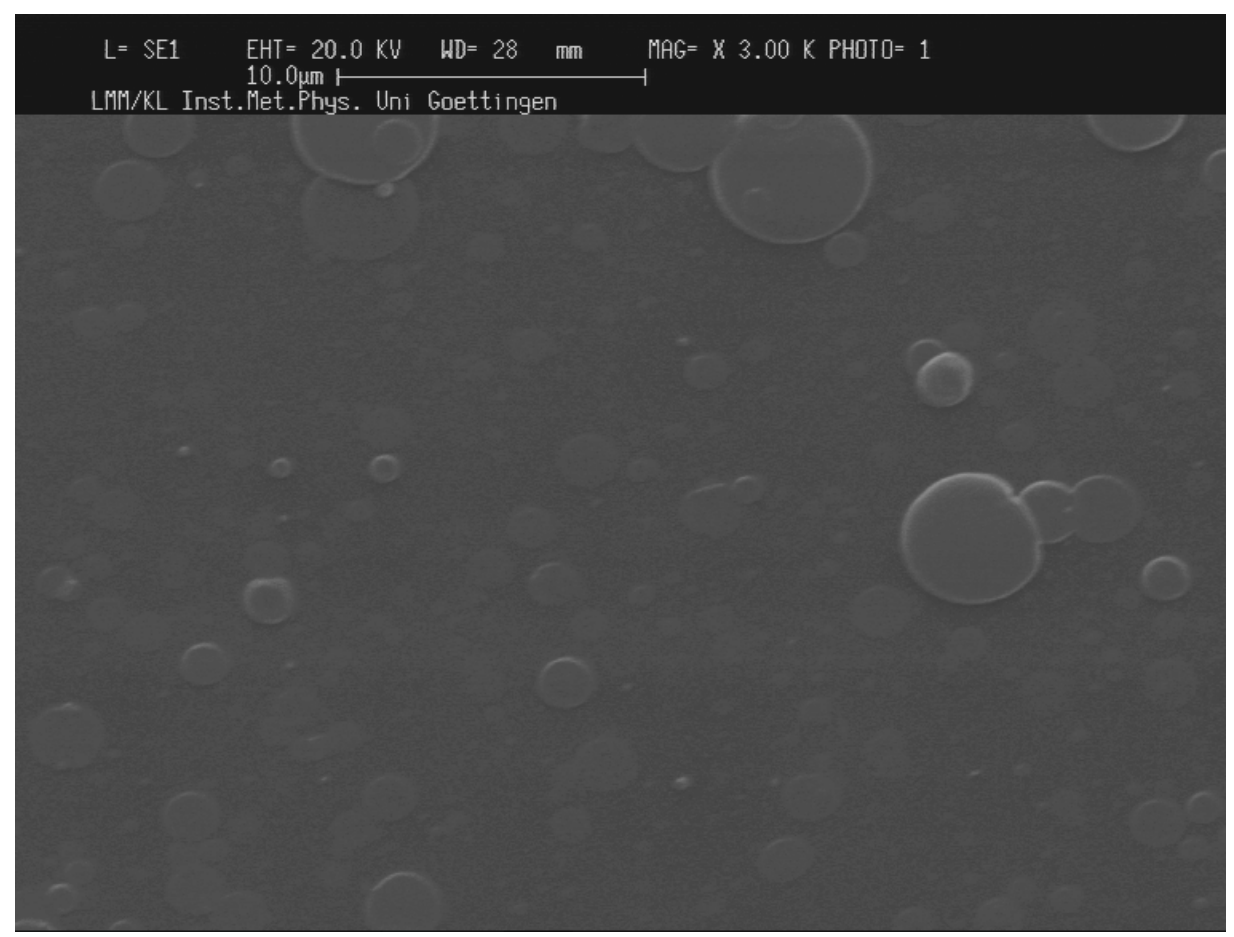

Abbildung 4.60: REM-Aufnahme von deponiertem PMMA, Maßstab $10 \mu \mathrm{m}$ (ca. 3000fache Vergrößerung).

Abbildung 4.60 zeigt eine typische Oberflächenstruktur von PMMA deponiert auf Silizium. Eine homogene Oberfläche ist zu erkennen, die allerdings mit einer hohen Anzahl an Tropfen, den sogenannten Droplets, überlagert ist. Hier kommt es zum Herauslösen von größeren Polymerteilen durch den Laser und diese werden in 
Tropfenform durch die Vakuumkammer geschleudert. Beim Auftreffen auf das Substrat entsteht dann diese Linsenform. Der Depositionsprozess ist zur Zeit noch nicht vollständig geklärt. Besonders interessant ist die Aufstellung eines Modells, das Aussagen über die Art der deponierten Ketten liefert. Werden die Polymere bis hin zur Ebene der Monomere durch den Laserpuls zerlegt und reorganisieren sich nach dem Auftreffen auf das Substrat, oder werden die Polymere als einzelne Ketten übertragen? Ist das Entstehen von Droplets ein Hinweis darauf, dass mehrer Polymerketten im Verbund übertragen werden? Bereits durchgeführte Untersuchungen an PMMA zeigen, dass sich die Molmassenverteilung des Polymer hin zu kleineren Molmassen ändert. ${ }^{[36]}$ Die Verwendung eines neuen Auslöseprozesses, genannt MAPLE (Matrix Assisted Pulsed Laser Evaporation) ${ }^{[44,45]}$ ermöglicht eine Deposition von PMMA mit größeren Molmassen. ${ }^{\text {[37] }}$

Interessant ist die Verwendung von weiteren Polymeren. Es wird im folgenden erstmals auf die Deposition von PE eingegangen, was aus unpolaren Monomeren aufgebaut ist und auch einige Erkenntnisse über das EMAA werden geschildert, da es sich dabei um ein sowohl aus unpolaren, als auch aus polaren Monomeren aufgebautes Copolymer handelt.

\subsubsection{Oberflächenstrukturanalyse von Polyethylen}

Polyethylen wird ebenfalls mit Hilfe der in Kapitel 3.1.2 beschriebenen Hochdruckanlage hergestellt. Bei dem verwendetem Polyethylen handelt es sich um eine bei $230{ }^{\circ} \mathrm{C}$ hergestellte Probe. Das Zahlenmittel der Molmasse $M_{\mathrm{n}}$ beträgt $2940 \mathrm{~g} \cdot \mathrm{mol}^{-1}$, das Gewichtsmittel $M_{\mathrm{w}}=11061 \mathrm{~g} \cdot \mathrm{mol}^{-1}$. Es wird eine REM-Aufnahme des direkt aus dem Abscheideprozess erhaltenen Homopolymers gemacht. Eine Gesamtübersicht über die resultierende Oberflächenstruktur liefert Abbildung 4.61. Es ist ein loser Zusammenschluss von Polymerteilen zu kleinen Partikeln zu erkennen. Um genauere Informationen über die Struktur dieser Partikel zu erhalten, wurde eine Bereichsaufnahme mit ca. 10000facher Vergrößerung gewählt (Abbildung 4.62). Die Oberfläche ist von Furchen durchzogen und weist weiche Kanten auf. Der Abscheidevorgang, also die Entspannung von einem Reaktionsdruck von 2000 bar auf Atmosphärendruck, hat hier einen strukturbestimmenden Einfluss. Das lässt sich bereits daran festmachen, dass das PE nach der Entspannung als Pulver vorliegt. Nach dem Aufschmelzen und definiertem Abkühlen unter Zuhilfenahme der DSC-Apparatur ergibt sich eine andere Struktur, die in Abbildung 4.63 und Abbildung 4.64 gezeigt ist. 


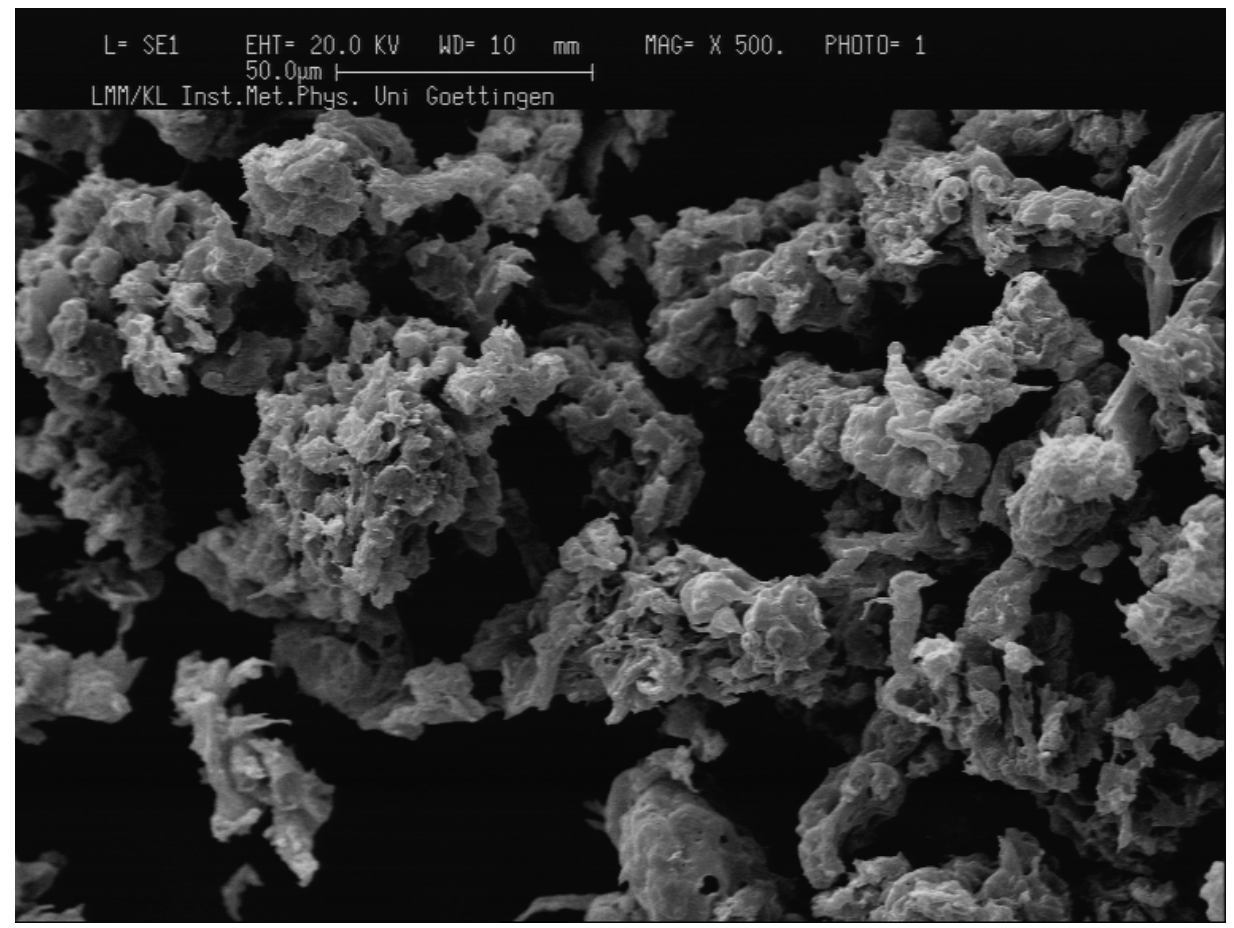

Abbildung 4.61: REM-Aufnahme von direkt nach dem Abscheidevorgang erhaltenem PE, Maßstab 50 um (ca. 500fache Vergrößerung).

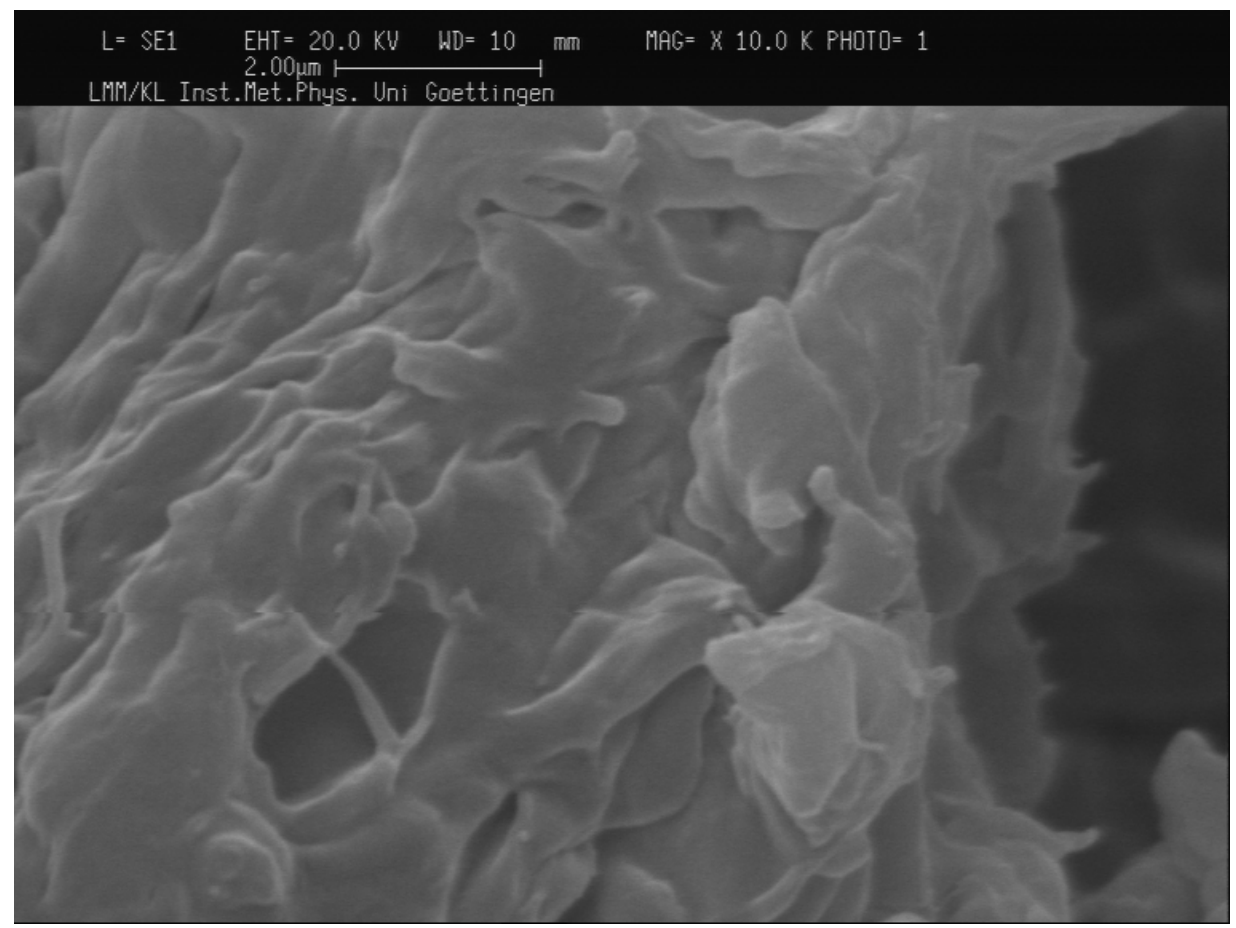

Abbildung 4.62: REM-Aufnahme von direkt nach dem Abscheidevorgang erhaltenem PE, Maßstab 2 um (ca. 10000fache Vergrößerung).

Nach dem Aufschmelzen der PE Proben und dem definiertem Abkühlen mit $5^{\circ} \mathrm{C} \cdot \mathrm{min}^{-1}$ wird die Probe direkt im DSC-Pfännchen mit Gold beschichtet und darauf hin auch im 
DSC-Pfännchen einer REM-Aufnahme unterzogen. Es liegt ein signifikanter Unterschied zu den direkt nach dem Abscheidevorgang erhaltenen Proben vor, wie Abbildung 4.63 verdeutlicht. Die Struktur zeigt sich deutlich schärfer. Das zu beobachtende Zackenmuster könnte auf kristalline Bereiche hindeuten, die während des definierten Abkühlvorgangs entstehen können. Weiterhin sind auch weiche Teilbereiche zu erkennen, was von einem amorphen Anteil herrühren kann. Die Kristallinität für diese Probe wurde von Becker ${ }^{[19]}$ auf ca. $60 \%$ bestimmt. Hier kann also von einem teilkristallinem Polymer gesprochen werden.

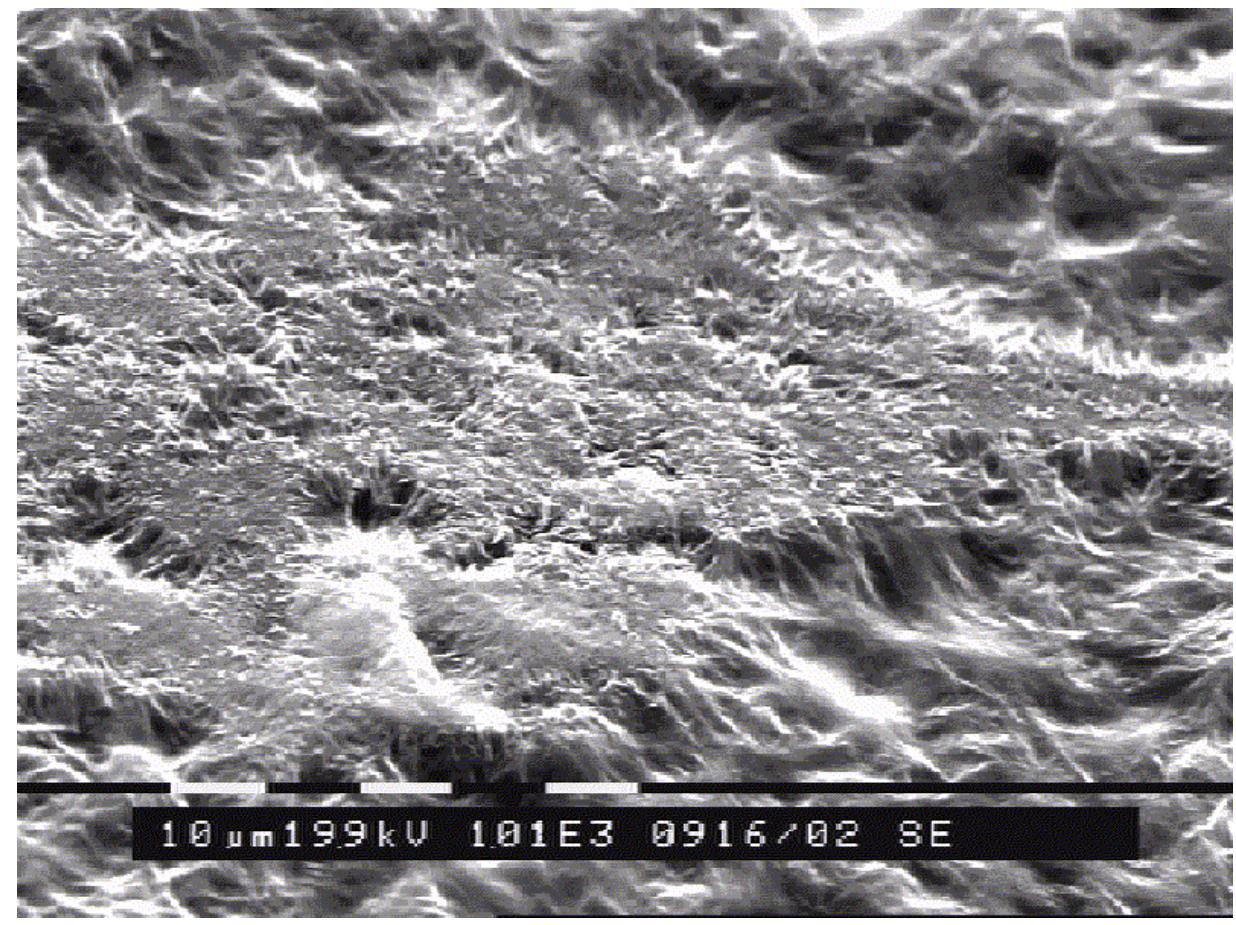

Abbildung 4.63: REM-Aufnahme von definiert abgekühltem PE (DSC $5{ }^{\circ} \mathrm{C} \cdot \mathrm{min}^{-1}$, Maßstab $10 \mu \mathrm{m}$ (ca. 1000fache Vergrößerung).

Die Eindrücke, die durch die Übersichtsaufnahme bei 1000facher Vergrößerung entstehen, lassen sich anhand einer Detailaufnahme (Abbildung 4.64) weiter festigen. Der interessante Bereich mit der kristallin anmutenden Struktur wurde hier mit einer Vergrößerung von 10000 abgebildet. Eine raue, scharfe Oberfläche kann beobachtet werden. Aufgrund dieser manifestierten Erkenntnisse ist es sinnvoll die Struktur von deponiertem PE zu untersuchen. 


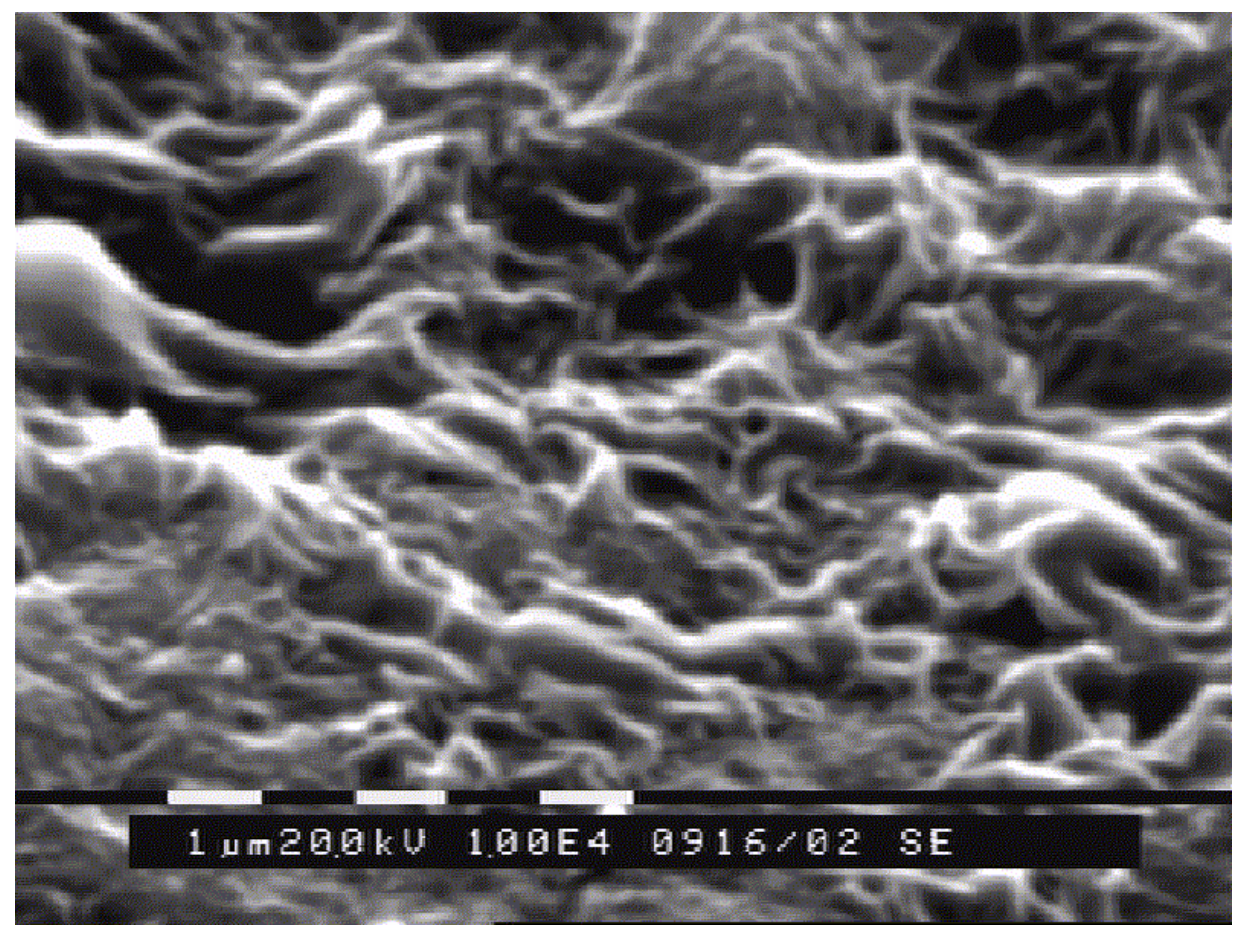

Abbildung 4.64: REM-Aufnahme von definiert abgekühltem PE (DSC $\left.\quad 5^{\circ} \mathrm{C} \cdot \mathrm{min}^{-1}\right)$, Maßstab 1 m m (ca. 10000fache Vergrößerung).

\subsubsection{Oberflächenstrukturanalyse von mittels PLD hergestellten PE-Schichten}

Es wurde PE in eine Tablettenform bei einer Temperatur von ca. $100{ }^{\circ} \mathrm{C}$ und einer Kraft von $40 \mathrm{kN}$ bei einem Durchmesser von $2.5 \mathrm{~cm}$ gepresst. Der Abkühlvorgang erfolgte mittels einer Wasserkühlung und mit nicht konstanten Kühlraten. Hier kann nicht von einer definierten Abkühlung gesprochen werden. Anfängliche Abkühlraten werden sich schätzungsweise im Bereich von 5 bis $10^{\circ} \mathrm{C} \cdot \mathrm{min}^{-1}$ bewegen. Die Kühlraten im Bereich der Zimmertemperatur werden, bedingt durch einen exponentiellen Abfall der Kühlrate, deutlich darunter liegen. Da das deponierte Material allerdings vor Erreichen des Substrats mittels der eingestrahlten Laserenergie abgetragen werden muss, sollte die auf dem Target (gepresste Tablette) vorliegende Struktur für die auf dem Substrat resultierende Struktur nicht ausschlaggebend sein. Zur Vermeidung von Hotspots sollte ein möglichst homogenes Target vorliegen. Das ist gewöhnlich der Fall, wenn eine klare und durchsichtige Tablette vorliegt. Es wurden jeweils 2000 Pulse während der PLD verwendet.

Einen Überblick über die resultierende Oberfläche von PE gibt Abbildung 4.65. Hier werden neben den bereits bekannten Droplets auch Fadenstrukturen gefunden. Die Droplets entstehen hier ebenfalls durch das Deponieren von Tröpfchen. Die Fadenstruktur 
wurde bei PMMA noch nie beobachtet. Es gibt zwei entscheidende Unterschiede zwischen den verwendeten PMMA und PE Proben. Bei PMMA handelt es sich um Polymer, das aus polaren Monomeren besteht, wo hingegen PE aus unpolaren Monomeren besteht. Das PE weist im Gegensatz zum PMMA eine Teilkristallinität auf. Welcher Effekt für die Fadenstruktur entscheidend wirken kann, wird in Kapitel 4.7.5 diskutiert.

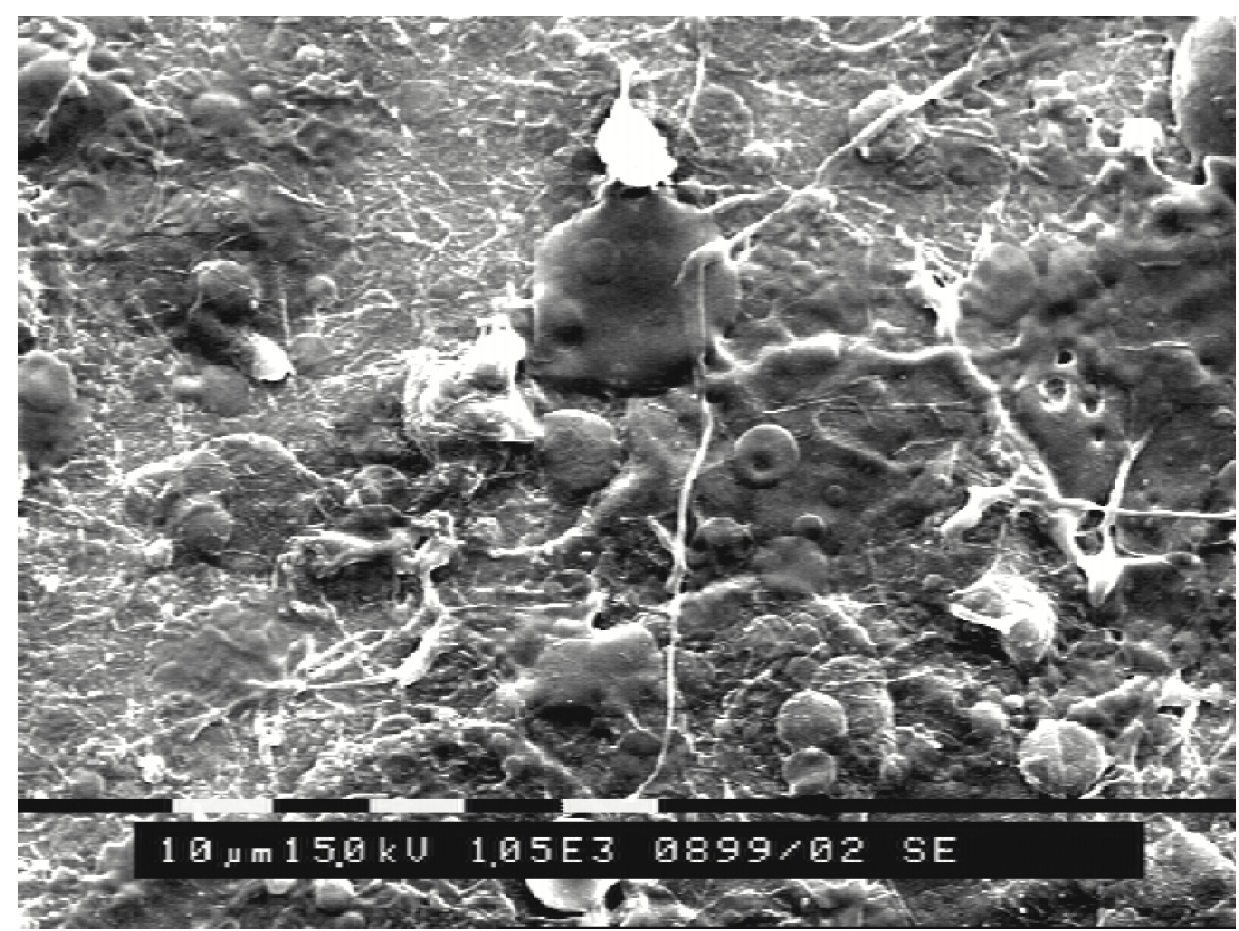

Abbildung 4.65: REM-Aufnahme von deponiertem PE, Maßstab $10 \mu \mathrm{m}$ (ca. 1000fache Vergrößerung).

Auch im Fall des deponierten PE ist eine Detailaufnahme (Abbildung 4.66) interessant. Die in der Übersichtsaufnahme nahezu gleichmäßige Grundstruktur (wie z. B. links unten am Bildrand $\mathrm{zu}$ sehen) die durch Fäden und Droplets überlagert ist, kann durch die Detailaufnahme widerlegt werden. Deutlich ist eine hügelige Landschaft zu erkennen, die sich aus kleinen Droplets zusammen setzt. Im Vergleich zum deponiertem PMMA kann also hier nicht von einer homogenen Grundstruktur gesprochen werden. 


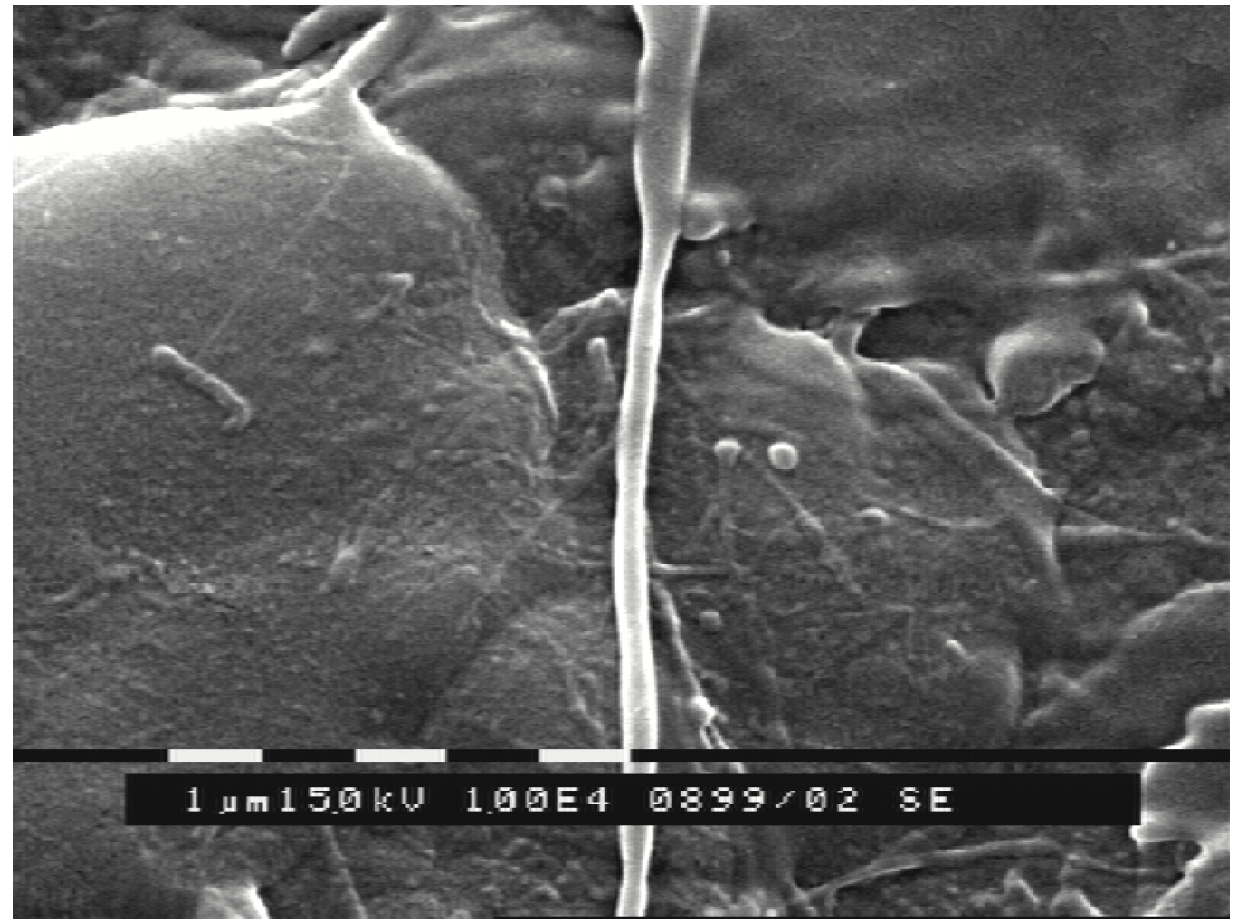

Abbildung 4.66: REM-Aufnahme von deponiertem PE, Maßstab $1 \mu \mathrm{m}$ (ca. 10000fache Vergrößerung).

\subsubsection{Oberflächenstrukturanalyse von mittels PLD hergestellten EMAA- Schichten}

Die Verwendung eines Copolymers für die PLD kann weitere Informationen über den Depositionsvorgang bringen. Es stellt sich die Frage, ob ein Copolymer, das sowohl aus unpolaren als auch aus polaren Monomereinheiten besteht, sich eher wie deponiertes PMMA, oder eher wie deponiertes PE verhält. Möglich wäre auch eine Mischung aus beidem. Das würde sich z. B. in der Form äußern, dass ein glatter Filmanteil (wie bei PMMA) mit einer relativ hohen Anzahl an Droplets oder sogar Fäden vorhanden ist (PE).

Dass es sich bei den mittels PLD hergestellten dünnen Schichten des Copolymers EMAA um genau diese Mischung der beiden bereits beschriebenen Homopolymere PE und PMMA handelt, zeigen die Abbildung 4.67 und Abbildung 4.68. Zur Herstellung der Schicht wurden 1000 Laserpulse verwendet.

In der Übersichtsaufnahme, Abbildung 4.67, lassen sich erhebliche Unterschiede der Untergrundstruktur zu dem System PE erkennen. Ein homogener Untergrund wird 
beobachtet (Abbildung 4.68). Die Oberfläche ist allerdings mit Droplets und Fäden versetzt.

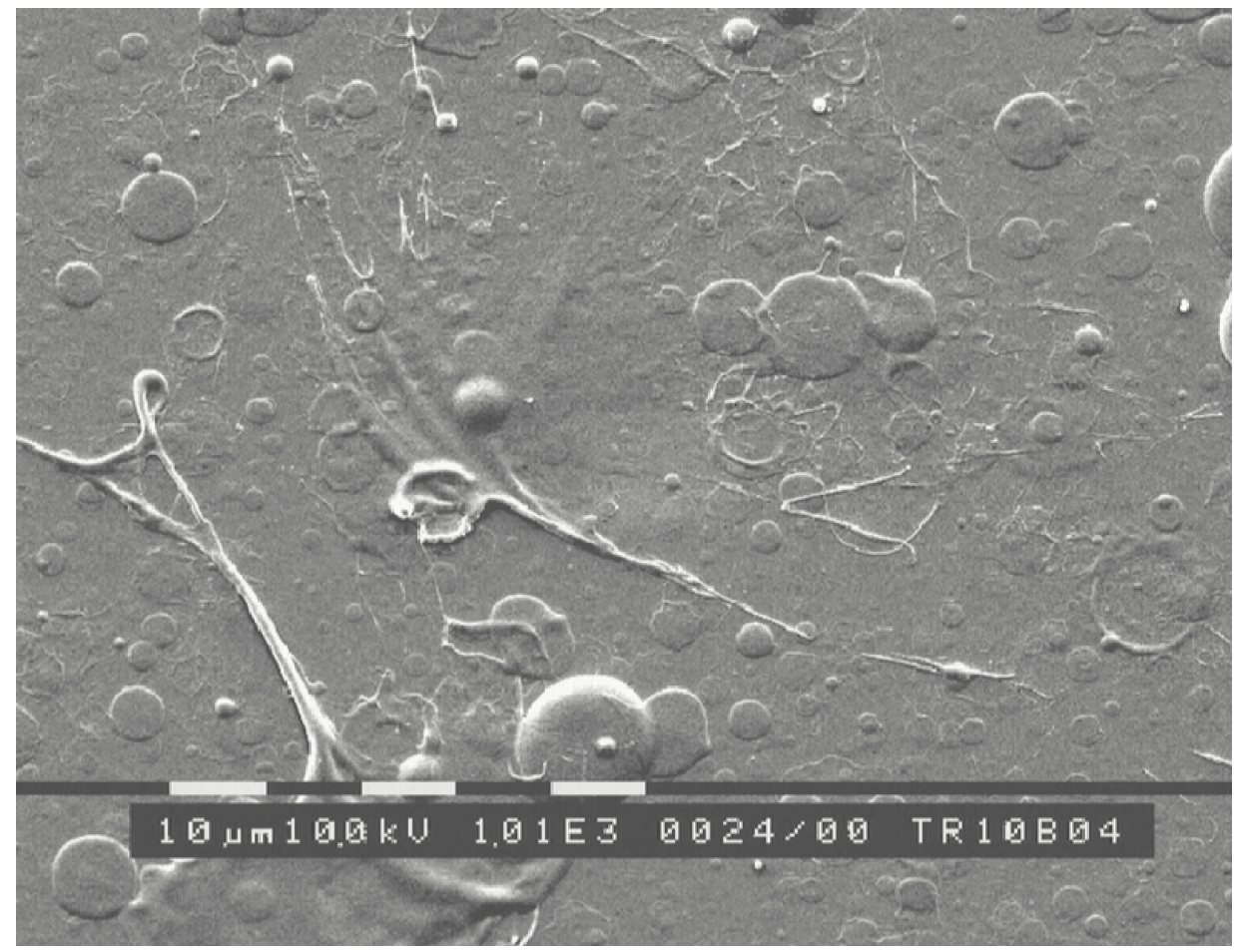

Abbildung 4.67: REM-Aufnahme von deponiertem EMAA, Maßstab $10 \mu \mathrm{m}$ (ca. 1000fache Vergrößerung).

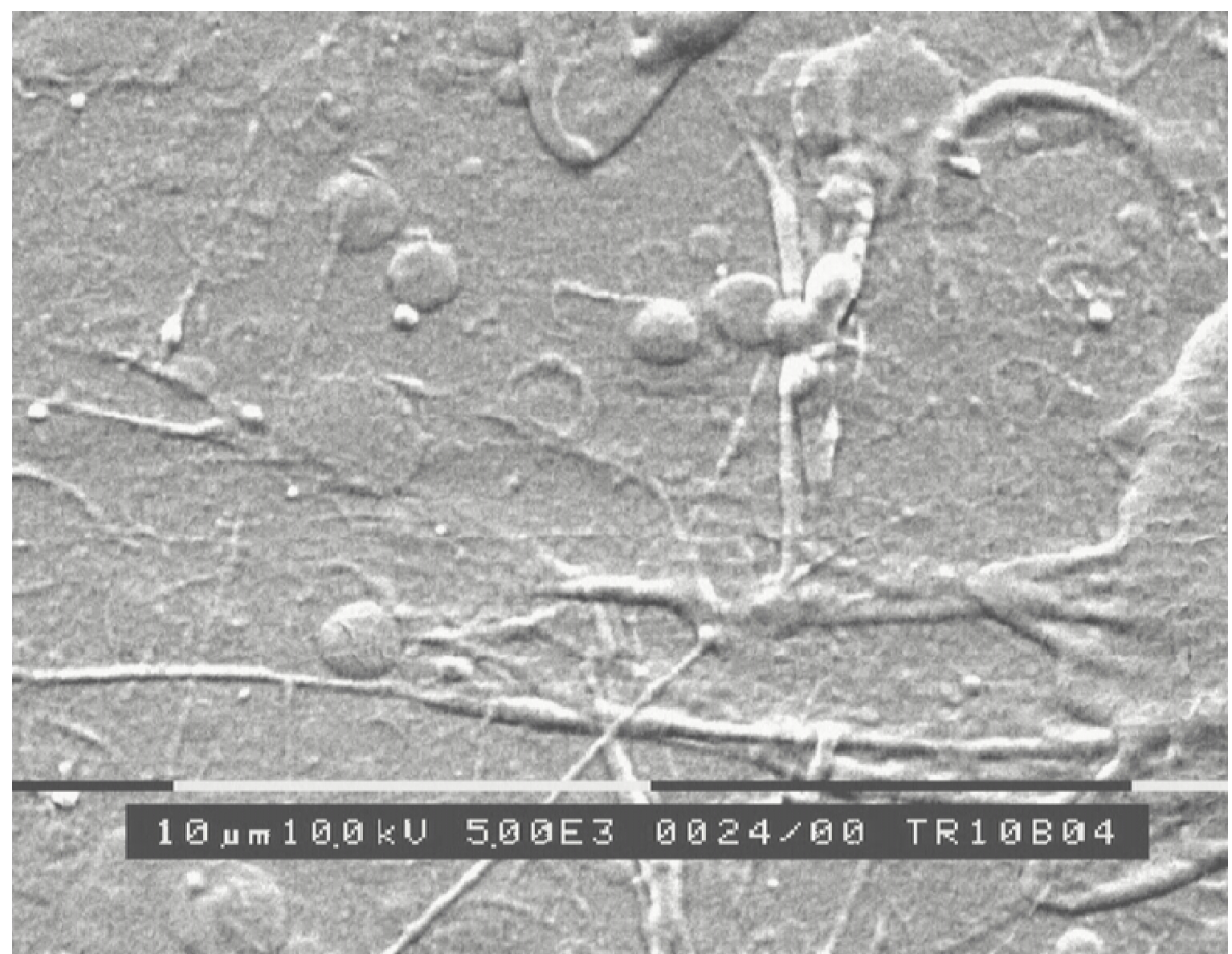

Abbildung 4.68: REM-Aufnahme von deponiertem EMAA, Maßstab $10 \mu \mathrm{m}$ (ca. 5000fache Vergrößerung). 
Bei dem verwendeten Copolymer EMAA 2.10 (Niederdruckprobe) handelt es sich um weitgehend amorphes Material. Die Existenz der Fäden bei beiden Proben lässt sich somit nur schwer anhand der Kristallinität der Proben erklären. Eine plausiblere Erklärung für die Fadenbildung liefert der hypothetische Ansatz, der zwischen polaren und unpolaren Polymeren differenziert. Die Anzahl der Fäden des deponierten Copolymers ist im Verhältnis zum deponierten PE deutlich zurück gegangen. Die Anzahl der Droplets pro Flächenabschnitt ist trotz der halben Anzahl an verwendeten Pulsen gestiegen. Untersuchungen an PMMA und PE zeigten, dass bei Verwendung einer höheren Pulsanzahl die Droplettintensität zunimmt. Da nun bei EMAA trotz geringerer Pulszahl im Vergleich zur PE-Deposition eine hohe Droplettanzahl beobachtet werden kann, spricht dies dafür, dass Droplets durch polare Bereiche im Copolymer entstehen können. Bei der verwendeten Probe handelt es sich um EMAA 2.10, der Gehalt an MAA in Copolymer beträgt 3.0 mol-\%. Somit besteht der Großteil des Polymers aus dem unpolaren Monomer Ethen. Das könnte für eine Fadenbildung durch unpolare Monomeranteile sprechen.

\subsubsection{Molmassenverteilung einer deponierten Copolymerprobe}

Frühere Untersuchungen der Molmassenverteilung von deponierten Polymerproben ergaben, dass für PMMA zum Beispiel meistens eine Kettenlängenverkürzung während des Depositionsprozesses auftreten. ${ }^{[36]}$ Ebenfalls ein Einfluss auf die nach der Deposition vorliegende Molmassenverteilung hat die Substrattemperatur. ${ }^{[46]}$ Die Experimente dieser Arbeit wurden bei Raumtemperatur durchgeführt. Interessant ist es nun, Informationen über die Molmassenverteilung der deponierten Copolymerproben zu erhalten. Da der gleiche PLD-Vorgang angewendet wurde, sollte hier ebenfalls eine erhebliche Verkürzung der deponierten Polymerketten auftreten. Interessant ist die Fragestellung, ob bei Copolymeren Material mit einem Molekulargewicht deponiert werden kann, welches der ursprünglichen Molmasse des Ausgangsmaterials entspricht. 


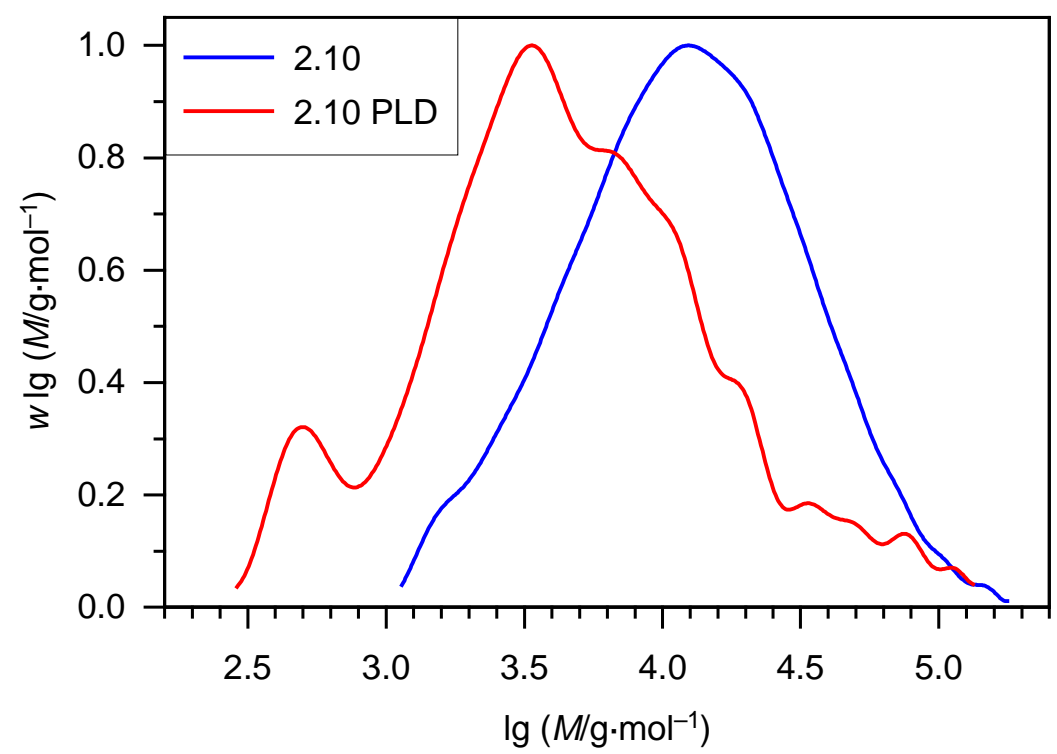

Abbildung 4.69: Vergleich der Molmassenverteilung von EMAA 2.10 (1300 bar) vor und nach der Deposition mittels PLD (Lösungsmittel TCB).

Abbildung 4.69 gibt Aufschluss darüber, dass ein signifikanter Unterschied der beiden bestimmten Molmassenverteilungen vorliegt. Die blaue Kurve zeigt eine unbehandelte Ausgangsprobe EMAA 2.10. Das Gewichtsmittel der Molmasse $M_{\mathrm{w}}$ beträgt $1.90 \cdot 10^{4} \mathrm{~g} \cdot \mathrm{mol}^{-1}$, das Zahlenmittel $M_{\mathrm{n}}=7.51 \cdot 10^{3} \mathrm{~g} \cdot \mathrm{mol}^{-1}$. Die daraus resultierende Polydispersität $(P D I)$ ist 2.53. Die rote Kurve zeigt die Molmassenverteilung einer mittels PLD erhaltenen Schicht der Probe EMAA 2.10. Die Probe wurde mit Hilfe des Lösungsmittels für die Hochtemperatur-GPC, Trichlorbenzol, unter erhöhter Temperatur vom Substrat abgelöst. Es lässt sich eine Verschiebung der Molmassenverteilung hin zu kleineren Molmassen beobachten. Das Gewichtsmittel beträgt nur noch $M_{\mathrm{w}}=9.67 \cdot 10^{3} \mathrm{~g} \cdot \mathrm{mol}^{-1}$, das Zahlenmittel $M_{\mathrm{n}}=2.33 \cdot 10^{3} \mathrm{~g} \cdot \mathrm{mol}^{-1}$. Die daraus resultierende Polydispersität steigt erheblich an und zeigt fast den doppelten Wert von PDI=4.16. Das Maximum der Molmassenverteilung liegt im Bereich von $10^{3.5} \mathrm{~g} \cdot \mathrm{mol}^{-1}$. Ein geringer Anteil an langkettigen Molekülen mit einem Molekulargewicht von $10^{4.5}$ bis $10^{5} \mathrm{~g} \cdot \mathrm{mol}^{-1}$ ist unter diesem Bedingungen bei einem Copolymer auf das Substrat übertragen worden. Die Häufigkeit dieser langkettigen Polymere im Vergleich zur Ausgangsprobe nimmt erheblich ab. Die Verteilung wird von mehreren lokalen Maxima und Schultern durchzogen. Das könnte dadurch erklärt werden, dass die Copolymerketten bevorzugt an einigen bestimmten Stellen aufbrechen könnten. Die Absorption des Laser mit einer Wellenlänge von $248 \mathrm{~nm}$ erfolgt vermutlich zum größten Teil in den Carbonylfunktionen der MAA-Einheiten, aber nicht ausschließlich, da ansonsten eine Deposition von PE nicht möglich wäre. Der genaue Mechanismus, der zum Aufspalten der Ketten führt, ist, wie oben beschrieben, noch nicht detailliert bekannt. Es 
wird vermutet, dass der Bruch der Kette aufgrund der Energieabsorption der Carbonylfunktionen in der unmittelbaren Umgebung dieser funktionellen Gruppe erfolgt. Unter Berücksichtigung dieses Aspektes kann die Existenz mehrerer Maxima der Molmassenverteilung bei einem deponierten Copolymer erklärt werden. Die Polymerkette bricht an den Stellen bevorzugt, wo Säuregruppen vorhanden sind.

$\mathrm{Da}$, wie oben gezeigt, auch das Homopolymer PE deponiert werden kann, ist nun auch die Existenz von hochmolekularen Polymeren verständlich. Aufgrund des geringen Säuregehalts von weniger als 3 mol-\% und einer Kettenlänge von im Mittel 252 Monomereinheiten sind durchschnittlich weniger als 8 Säuregruppen pro Kette vorhanden. Es kann vorkommen, dass eine Kette gar keine Säureeinheiten oder diese an den Kettenenden trägt, was zu einer Übertragung von längeren Ketten führen kann, die nicht weiter aufgespaltet werden. Das bestätigen auch die Aufnahmen des Rasterelektronenmikroskops. Hier werden ebenfalls Strukturbereiche gefunden, die denen des Homopolymers PE stark ähneln.

Die Existenz eines niedermolekularen Maximums bei einer Molmasse im Bereich von $10^{2.7} \mathrm{~g} \cdot \mathrm{mol}^{-1}$ ist wie bei bereits untersuchten PMMA-Proben ${ }^{[46]}$ auf Oligomere zurückzuführen. Das entspricht einer Molmasse von ca. $500 \mathrm{~g} \cdot \mathrm{mol}^{-1}$. Da die verwendete Hochtemperatur-GPC im niedermolekularen Bereich über eine nicht ausreichende Auflösung und Kalibration verfügt, kann hier aber keine genaue Zuordnung getroffen werden. Es kann sich eventuell auch um Monomereinheiten der Säure oder um sehr kurze Ketten des Copolymers handeln.

\subsubsection{Diskussion}

Der Grundgedanke dieser Arbeit ist die Unterscheidung von statistisch und nicht-statistisch aufgebauten Copolymeren anhand ihrer Eigenschaften. Da allerdings bereits der Einfluss von polaren und unpolaren Monomeren zu einer komplizierten Oberflächenstruktur führen, erscheint es nicht sinnvoll, die Experimente zum jetzigen Zeitpunkt, beim aktuellen Stand der Forschung auf Hochdruckproben auszubauen. Es bedarf hier der weiteren systematischen Klärung an möglichst einfachen Systemen. Es wäre wünschenswert PLD an Copolymeren durchzuführen, bei denen der Gehalt eines polaren Monomers von 0 bis $100 \%$ in beliebiger Abfolge zu variieren ist. Durch diese Art von Experimenten sollte die aufgestellte Hypothese, dass sich deponierte polare Polymere durch eine homogene Grundstruktur überzogen mit Droplets und unpolare Polymer durch ein Gemisch aus Droplets und Fäden auszeichnen, bekräftigt werden oder widerlegt werden können. 


\subsection{Vergleich der Systeme Ethen/Methacrylsäure und Ethen/Methyl- methacrylat}

Das in dieser Arbeit verwendete Comonomer Methacrylsäure besitzt durch das Vorhandensein der Säurefunktion die Möglichkeit Wasserstoffbrückenbindungen auszubilden. Bei der Copolymerisation von EMAA nahe der Phasengrenze wird aufgrund dieser Wechselwirkungensmöglichkeit eine nicht-statistische Anordnung von Säuregruppen erwartet. Die Säuregruppen besitzen anscheinend eine höhere Anzahl an Säuregruppen in direkter Nachbarschaft als einer statistischen Verteilung entsprechen würde. Mittels der Analyseverfahren DSC und DMA werden erhebliche Unterschiede für Hoch- und Niederdruckproben aufgezeigt. Auch für die parallel zu dieser Arbeit durchgeführten Experimente für Acrylsäurecopolymere (EAA) wurden diese Tendenzen gefunden.

Interessant ist es nun, ob ein Referenzsystem, das keine Säurefunktion besitzt, ebenfalls Unterschiede für Proben, die nahe der Phasengrenze synthetisiert sind, zeigt. Als sehr gut geeignetes Vergleichssystem für EMAA-Copolymere eignet sich das System Ethen/Methylmethacrylat (EMMA). Es handelt sich hier bei dem Comonomer Methylmethacrylat (MMA) um die methylveresterte Form der Methacrylsäure. Somit besteht keine Möglichkeit zur Ausbildung von Wasserstoffbrückenbindungen beim Comonomer. Bei Zurückführung der für EMAA beobachteten Effekte auf die Säurefunktion sollten sich für die veresterte Form (EMMA) keine Auswirkungen auf den Kettenaufbau während der Synthese ergeben.

Um dies anhand von Versuchen belegen zu können, wurden Copolymerisationen von Ethen und Methylmethacrylat durchgeführt. Da bereits Informationen der Säurecopolymerisation vorlagen, war bekannt, dass die Unterschiede zwischen den Hochund Niederdruckproben bei hohen Synthesetemperaturen besonders gut ausgeprägt sind. Es wurde eine Synthese von EMMA bei einer Reaktionstemperatur von $270{ }^{\circ} \mathrm{C}$ durchgeführt (EMMA 6). Die Hochdruckprobe wurde bei 2000 bar hergestellt. Die Phasengrenze wurde unter den gegebenen Reaktionsbedingungen bei einem Druck von 1070 bar beobachtet $\left(\right.$ siehe auch ${ }^{[47]}$ ). Die homogene Synthese der Niederdruckproben wurde bei 1200 bar durchgeführt. Der Gehalt an MMA im Dosierstrom beträgt $f_{\mathrm{MMA}}=0.0130$, ist somit im Vergleich zu den Säurecopolymeren relativ hoch und liegt nahe bei dem höchsten verwendeten Fluss (Probenreihe EMAA 3, $f_{\mathrm{MAA}}=0.020$ ). Es ergeben sich Copolymere mit einem MMA-Gehalt von $F_{\mathrm{MMA}}=0.06$. Somit wurden Bedingungen gewählt, die falls ein Effekt vorliegen sollte, diesen am deutlichsten zeigen sollten. 
Abbildung 4.70 zeigt die nach Normierung auf die Probenmasse erhaltenen DSCThermogramme.

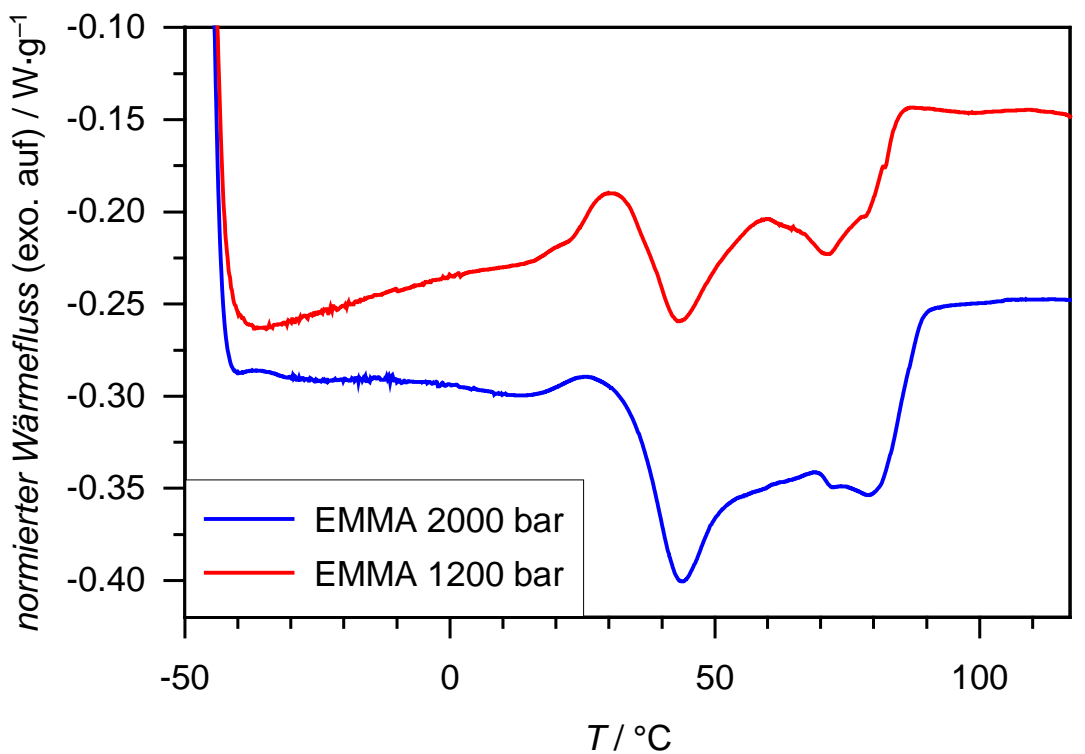

Abbildung 4.70: DSC-Thermogramm für bei unterschiedlichen Drücken bei $270{ }^{\circ} \mathrm{C}$ hergestellte EMMA 6 Copolymere. Der Methacrylatgehalt im Dosierstrom beträgt $f_{M M A}=0.0130$, was zu $F_{M A A}=0.06$ führt. Werte auf Probenmasse normiert.

Die Hochdruckprobe weist im Bereich von -45 bis $0{ }^{\circ} \mathrm{C}$ keine signifikanten Signale auf. Im Bereich von $5{ }^{\circ} \mathrm{C}$ bis $20{ }^{\circ} \mathrm{C}$ kann eine Minimumbildung im Wärmefluss beobachtet werden. $\mathrm{Ab}$ einer Temperatur von $25^{\circ} \mathrm{C}$ beginnt der Glasübergang endotherm zu verlaufen. Für die Niederdruckprobe ist ein stetiger Anstieg des Wärmeflusses im Bereich von -45 bis $15^{\circ} \mathrm{C}$ zu beobachten. Dies kann mit Hilfe der Temperaturabhängigkeit der spezifischen Wärmekapazität, $c_{\mathrm{p}}$, erklärt werden. Wie in Kapitel 4.10 .5 erwähnt, wird für bei niedrigerem Druck hergestellte Proben eine geringere Kristallinität gefunden. Der amorphe Anteil der Niederduckproben ist somit höher und offensichtlich liegt für diese Proben eine stärker ausgeprägte Temperaturabhängigkeit von $c_{\mathrm{p}}$ vor, was sich im kontinuierlich ansteigendem Wärmefluss bei Temperaturen unterhalb des Glasübergangs äußert. Diese Ergebnisse decken sich mit den mittels DSC in Kapitel 4.10 gemachten Beobachtungen und den daraus resultierenden Abschätzungen für die Kristallinität. Die Auswirkung des Synthesedrucks auf die Kristallinität der Copolymerproben ist somit kein spezifischer Effekt für Säuresysteme als Comonomer sondern kann auch bei Acrylaten beobachtet werden. 
Wie bereits erwähnt, ist für diese Arbeit von besonderer Bedeutung, ob sich Hoch- und Niederdruckproben für das System EMMA signifikant unterscheiden. Um einen besseren Eindruck vom Signalverlauf im interessanten Bereich, der bei tieferen Temperaturen als der Glasübergang ( $\alpha$-Übergang) liegt, zu bekommen, wurde eine Normierung mit Hilfe einer Geradengleichung (-28 bis $0{ }^{\circ} \mathrm{C}$ ), analog $\mathrm{zu}$ dem in Kapitel 4.10 vorgestellten Verfahren, über den gesamten Temperaturbereich vorgenommen. Die erhaltenen Thermogramme zeigt Abbildung 4.71. Die beiden Proben zeigen einen nahezu identischen Verlauf des Wärmeflusses bei Temperaturen unterhalb der Glasübergangs. Ein Resultat dieser Analyse ist, dass sich Hoch- und Niederdruckproben von EMMA nicht signifikant im besagten Temperaturintervall unterscheiden. Es kommt allerdings zur Ausbildung eines lokalen Minimums im Temperaturbereich, in dem für die Säurecopolymere ein $\beta$-Übergang beobachtet wurde. Aufgrund der hohen Manteltemperatur von $260{ }^{\circ} \mathrm{C}$, der höheren Verweilzeit der Reaktionsmischung im Reaktor, da diesmal im Gegensatz zu allen anderen Messungen anstatt 1800 lediglich $1000 \mathrm{~g} \cdot \mathrm{h}^{-1}$ Ethen verwendet wurden, ist ein direkter Vergleich mit den Säurecopolymeren schwierig. Aus diesem Grund wurden weitere Experimente unter nahezu gleichen Bedingungen wie die Säurecopolymerisationen durchgeführt (Abbildung 4.72). Die Ausbildung des in Abbildung 4.71 gezeigten Minimums bei ca. $15^{\circ} \mathrm{C}$ ist höchstwahrscheinlich auf Verzweigungen im Copolymer zurückzuführen und kann mittels des in Kapitel 5.2.1 erläuterten „Geometric Confinements“ erklärt werden. Da sich die Hoch- und Niederdruckprobe nicht signifikant unterscheiden, kann ein CC (siehe Kapitel 5.2.1) weitestgehend ausgeschlossen werden. 


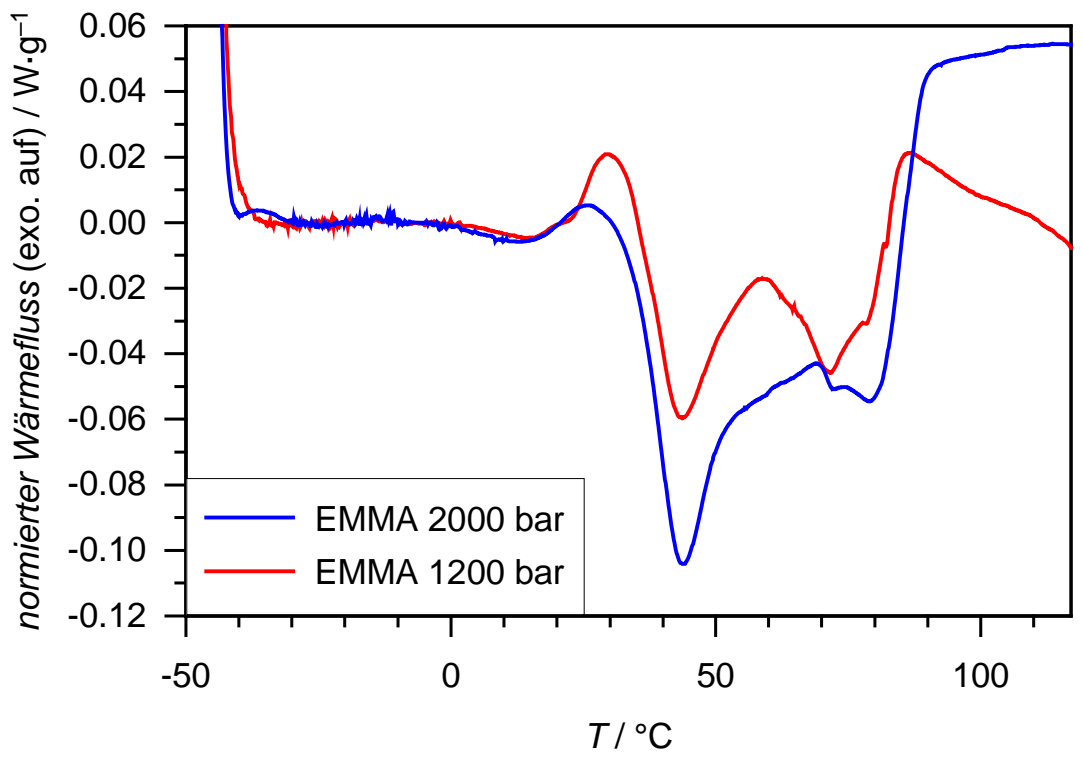

Abbildung 4.71: DSC-Thermogramm für bei unterschiedlichen Drücken bei $270{ }^{\circ} \mathrm{C}$ hergestellte EMMA 6 Copolymere. Der Methacrylatgehalt im Copolymer beträgt $F_{M A A}=0.06$. Werte auf Temperaturbereich unterhalb des Glasübergangs normiert.

Es wurde eine weitere EMMA-Copolymerisation unter nahezu identischen Bedingungen wie bei der Experimentreihe EMAA 2 durchgeführt, allerdings mit einem höheren Comonomergehalt im Copolymer. Die entsprechenden Parameter (EMMA 8) sind dem Anhang zu entnehmen. Für den Fall, dass sich ein CC ergibt, so sollte es unter diesen Bedingungen am deutlichsten zu beobachten sein. Am Ende dieses Versuchstages kam es aufgrund der anspruchsvollen Synthesebedingungen zu einer Zersetzungsreaktion im Reaktor, beim Versuch, den Umsatz für eine Niederdruckprobe weiter zu steigern. Diese Zersetzung hatte den Abbruch des Experiments zur Folge.

Der wesentliche Unterschied $\mathrm{zu}$ den in Abbildung 4.70 und Abbildung 4.71 gezeigten (EMMA 6) und den in Abbildung 4.72 dargestellten Copolymeren (EMMA 8) ist die Manteltemperatur. Bei EMMA 6 beträgt die Manteltemperatur $260{ }^{\circ} \mathrm{C}$ wo hingegen sie bei EMMA 8 lediglich $240{ }^{\circ} \mathrm{C}$ beträgt. Deswegen ist bei einer Reaktionstemperatur von $270{ }^{\circ} \mathrm{C}$ fast der doppelte Umsatz zu erreichen. Des Weiteren wurde der Ethenfluss von $1000 \mathrm{~g} \cdot \mathrm{h}^{-1}$ auf die Menge von $1800 \mathrm{~g} \cdot \mathrm{h}^{-1}$ erhöht. Dieser Dosierstrom fand bei allen Säurecopolymerisationen Verwendung. Die resultierenden Thermogramme dieser Proben sind Abbildung $4.72 \mathrm{zu}$ entnehmen. 


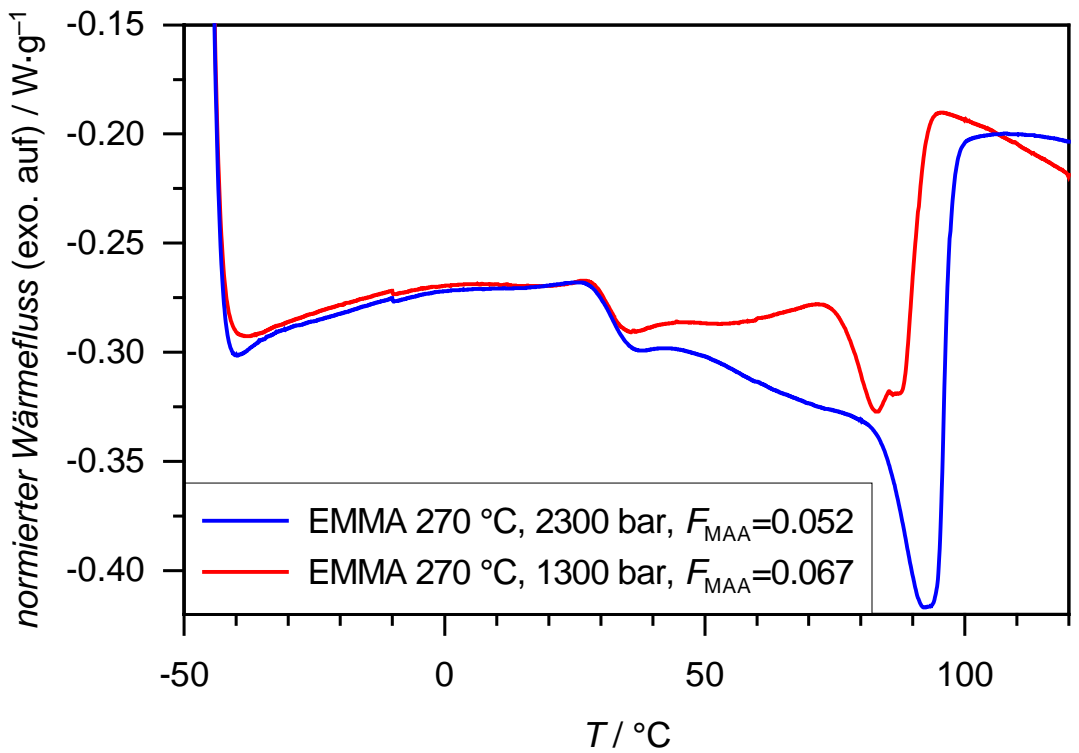

Abbildung 4.72: DSC-Thermogramm für bei unterschiedlichen Drücken bei $270{ }^{\circ} \mathrm{C}$ hergestellte EMMA Copolymere. Der Methacrylatgehalt im Dosierstrom beträgt $f_{M M A}=0.0101$. Werte auf Probenmasse normiert.

Die Copolymere, die unter ähnlichen Bedingungen wie die Säurecopolymere EMAA 2 und EMAA 3 hergestellt worden sind, zeigen bei Temperaturen, die unterhalb des Glasübergangs ( $\alpha$-Übergang) liegen, keine signifikante Ausbildung eines zusätzlichen Glasübergangs ( $\beta$-Übergang). Die leichte Plateaubildung, die bei tieferen Temperaturen als der $\alpha$-Übergang zu beobachten ist, kann auf Verzweigungen im Copolymer zurückgeführt werden (siehe Kapitel 5.2.1). Die DSC-Kurven wurden lediglich auf das verwendete Probengewicht normiert und zeigen nahezu einen deckungsgleichen Verlauf im interessanten Temperaturbereich unterhalb der Glastemperatur für Hoch- und Niederdruckproben. Ein nicht-statistischer Aufbau der Niederdruckproben oder ein CC kann nicht beobachtet werden.

\section{Diskussion:}

Die an EMMA-Copolymeren durchgeführten DSC-Messungen ergeben keinen Hinweis auf einen nicht-statistischen Aufbau der Niederdruckproben. Die Thermogramme für Hoch und Niederdruckproben zeigen im Temperaturbereich von 0 bis $25^{\circ} \mathrm{C}$ einen nahezu gleichen Verlauf. Die Annahme, dass der Einfluss der Säuregruppen bei Synthesen nahe der Phasengrenze zu einem weiteren Übergang führt, der durch das CC entsteht, wird durch die Messungen an dem Referenzsystem EMMA bekräftigt. 
Für auf das bereits verwiesene System EAA bietet sich eine Referenzmessung unter Verwendung von Ethen/Methylacrylat-Copolymeren an. Die Resultate werden in ${ }^{[20]}$ aufgeführt. 


\section{Literaturverzeichnis Kapitel 4}

[1] J. Hachenberg, Dissertation, Göttingen (2006)

[2] M. D. Lechner, K. Gehrke, E.H. Nordmeier, „Makromolekulare Chemie“, 3. Auflage, Birkhäuser (2003)

[3] M. Müller, Dissertation, Göttingen (2005)

[4] B. Steisel, Diplomarbeit, Göttingen (2004)

[5] T. Arita, M. Buback, O. Janssen, P. Vana, Macromol. Rapid Commun. 25 (2004) 1376

[6] E.-M. Borschel, Dissertation, Göttingen (1987)

[7] H. Latz, Diplomarbeit, Göttingen (1999)

[8] United States Patent 4,248,990

[9] G. Luft, R. Steiner, Chemiker Zeitung, 95 (1971) 11-15

[10] J. R. MacCallum, Thermochimica Acta, 96(2) (1985) 275

[11] I. Low, G. Paglia, C. Shi, J. Appl. Polym. Sci. 70 (1998) 2349

[12] S. G. Roos, Dissertation, Mainz (1998)

[13] L. H. Sperling, "Introduction to physical polymerscience“, second edition, WileyInterscince (1992)

[14] N. G. McCrum, B. E. Read, G. Williams, "Anelastic and dielectric effects in polymeric solids", John Wiley \& Sons (1967)

[15] K. Binder, W. Kob, "Glassy materials and disordered solids“, World Scientific (2005)

[16] M. Doi, S. F. Edwards, "The theory of polymer dynamics", Oxford Science Publications, Clarendon Press (1988)

[17] D. Richter, "Polymer dynamics“, Kap. C2 in "Soft matter: complex materials on mesoscopic scales“, 33. IFF-Ferienkurs, Forschungszentrum Jülich (2002)

[18] G. Luft, R. Kämpf, H. Seidl, Die Angew. Makro. Chem. 108 (1982), 203

[19] F. Becker, Dissertation, Göttingen (2006)

[20] U. Nergui, Dissertation, Göttingen (voraussichtlich 2008)

[21] H. Teichler, Phys. Rev. E 71 (2005) 031505

[22] K. Vollmayr-Lee, A. Zippelius, Phys. Rev. E 72 (2005) 041507

[23] M. Demetriou, J. Harmon, M. Tao, G. Duan, K. Samwer, W. Johnson, Physical Review Letters 97 (2006) 65502

[24] M. Demetriou, W. Johnson, Acta Materialia 52 (2004) 3403 
[25] L. Ravichandran, W. Johnson, Acta Materialia 51 (2003) 3429

[26] P. Lunkenheimer, A. Loidl, Chemical Physics 284(1-2) (2002) 205

[27] P. Rösner, J. Hachenberg, K. Samwer, R. Wehn, P. Lunkenheimer, A. Loidl, E. Sueske, T. Scharf, H.-U. Krebs, New J. Phys. 8 (2006) 89

[28] N.H. Ahmad, F. Heatley, P. A. Lovell, Macromolecules 31 (1998) 2822

[29] T. Fukuda, K. Kubo, Y.D.Ma, Prog. Polym. Sci. 17 (1992) 875

[30] E. F. McCord, W. H. Shaw, R. A. Hutchinson, Macromolecules 30 (1997) 246

[31] D. B. Chrisey (Hrsg.), G. K. Hubler (Hrsg.), "Pulsed Laser Deposition Of Thin Films", New York, Wiley Interscience Publication (1994)

[32] T. Scharf, J. Faupel, K. Sturm, H.-U. Krebs, J. Appl. Phys. 94 (2003) 4273

[33] H.-U. Krebs, M. Weisheit, J. Faupel, E. Süske, T. Scharf, C. Fuhse, M. Störmer, K. Sturm, M. Seibt, H. Kijewski, D. Nelke, E. Panschenko, M. Buback, Adv. in Solid State Phys. 43 (2003) 505

[34] H.-U. Krebs, S. Fähler, O. Bremert, Appl. Surf. Sci. 86 (1995) 86

[35] M. Störmer, H.-U. Krebs, J. Appl. Phys. 78 (1995) 7080

[36] E. Süske, Dissertation, Göttingen (2005)

[37] T. Scharf, Dissertation, Göttingen (2006)

[38] G. B. Blanchet, P. Cotts, C. R. Fincher Jr, J. Appl. Phys. 88 (2000) 2975

[39] S. G. Hansen, Robitaille: J. Appl. Phys. 64 (1988) 2122

[40] D. B. Chrisey, A. Piqué, R. A. McGill, J. S. Horwitz, B. R. Ringeisen D. M. Bubb, P. K. Wu, Chem. Rev. 103 (2003) 553

[41] P. E. Dyer, Appl. Phys. A 77 (2003) 167

[42] S. Küper, M. Stuke, Appl. Phys. A 49 (1989) 211

[43] R. Srinivasan, J. Appl. Phys. 73 (1993) 2743

[44] A. Piqué; R. A. McGill, D. B Chrisey, B. J. Spargo, J. H. Callahan, R. W. Vachet, R. Chung, M. A. Bucaro, D. Leonhardt, Thin Solid Films 355-356 (1999) 536

[45] P. K. Wu, B. R. Ringeisen, D. B. Krizman, C. G. Frondoza, M. Brooks, D. M. Bubb, R. C. Y. Auyeung, A. Piqué, B. Spargo, R. A. McGill, D. B. Chrisey, Rev. Sci. Instrum. 74 (2003) 2546

[46] E. Süske, T. Scharf, T. Junkers, M. Buback, H.-U. Krebs, J. Appl. Phys. 100 (2006) 14906

[47] H. Latz, Dissertation, Göttingen (2004) 


\section{Konzept des ,Chemical Confinements“}

\subsection{Definition und Ursachen des, „Chemical Confinements“}

Das „Chemical Confinement“, zu deutsch chemische Behinderung, wurde bereits bei Hachenberg ${ }^{[1]}$ eingeführt und in ${ }^{[2]}$ beschrieben. Die Namensgebung geschieht in Anlehnung an einen Effekt, der „Geometric Confinement“ genannt wird. ${ }^{[3]}$ Bei „Geometric Confinement“ wirken sich Änderungen der Probenform auf die Beweglichkeit der Polymere aus. Die zum Beispiel durch Pressen von dünnen Folien in das Polymer eingebrachten Verhakungspunkte verursachen zusätzliche Relaxation, unter anderem in der DES. ${ }^{[4]}$

Hoch- und Niederdruckproben unterscheiden sich signifikant im Kettenaufbau. ${ }^{[5]}$ Für Proben, die unter Bedingungen nahe der Phasengrenze synthetisiert wurden (Niederdruckproben), liegt eine nicht-statistische Verteilung der Säuregruppen über das Copolymer vor. Bei Hochdruckproben wird eine statistische Verteilung beschrieben. ${ }^{[5]}$ Zur Veranschaulichung der verwendeten Proben ist in Abbildung 5.1 das Phasenverhalten für EMAA gegeben ${ }^{[6]}$ und die hergestellten Proben wurden entsprechend eingetragen. Aus diesem Diagramm ergibt sich, dass einige Niederdruckproben im heterogenen Bereich hergestellt sein müssten. Das von Latz ${ }^{[6]}$ bestimmte Phasendiagramm schildert aber den ungünstigsten Fall. Das bedeutet, es wurden verschiedene Gewichtsanteile an Polymer eingewogen ${ }^{[6]}$ und die Messreihe veröffentlich, bei der die Maximalwerte für Druck und Temperatur gefunden wurde. Die in dieser Arbeit hergestellten Proben weisen einen Gehalt an Polymer in der Monomermischung auf, der nur $\mathrm{zu}$ niedrigeren Druck- und Temperaturwerten beim beobachteten Trübungspunkt führen kann, nicht $\mathrm{zu}$ höheren Werten. Es kann somit davon ausgegangen werden, dass die Copolymere im homogenen Bereich hergestellt worden sind (siehe Kapitel 3.1.2 und 3.1.7.3), bis auf wenige beschriebene Ausnahmen, die Kapitel 3.1.7.3 aufzeigt. Es handelt sich dabei um Niederdruckproben, die bei 2300 bar synthetisiert wurden. Die Temperaturen für diese Proben EMAA 30.3 (2300 bar) und 31.2 (2300 bar) mussten für die Auftragung auf $225^{\circ} \mathrm{C}$ und $215{ }^{\circ} \mathrm{C}$ geschätzt werden. Für alle anderen Proben wurden Mittelwerte aus den in Tabelle 4.1 angegebenen Temperaturen gebildet. 


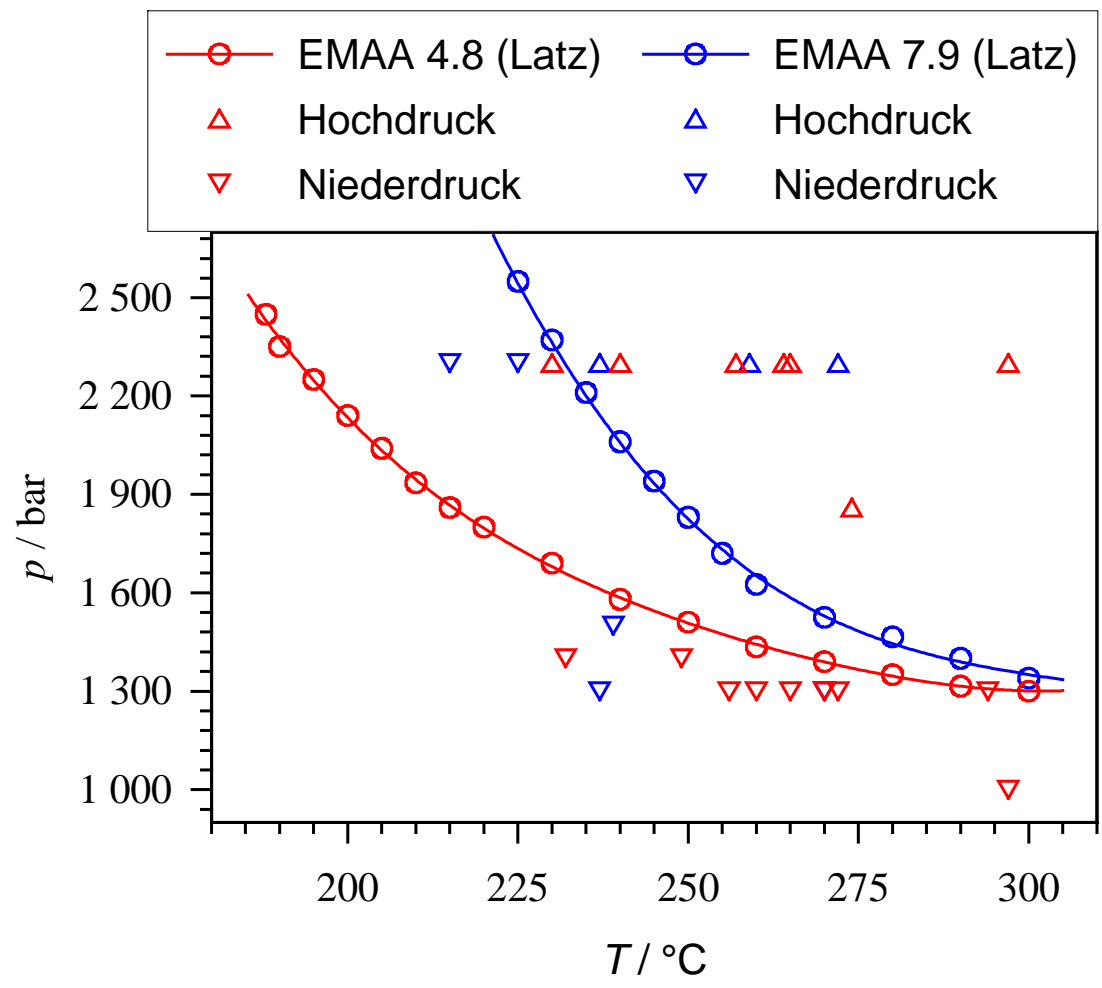

Abbildung 5.1: Phasendiagramm für EMAA Copolymere. Die Daten EMAA 4.8 und EMMA 7.9 stehen für Copolymere mit einem MAA-Gehalt im Copolymer von 4.8 und 7.9 mol-\% (runde Symbole) und sind der Literatur entnommen. ${ }^{[6]}$ Die rotgekennzeichneten Proben sind von ihrem MAAGehalt eher den Proben mit 4.8 mol-\% MAA und die blauen Proben eher 7.9 mol-\% MAA zuzuordnen. Die nach oben zeigenden Dreiecke kennzeichnen Hochdruckproben und die nach unten zeigenden Dreiecke Niederdruckproben. (Probendetails: siehe Tabelle 4.1)

Das Phasendiagramm zeigt, dass für viele Reaktionstemperaturen jeweils Hoch- und Niederdruckproben vorhanden sind. Die Niederdruckproben wurden sehr nah an der Phasengrenze synthetisiert und die Hochdruckproben weisen einen erheblichen Abstand zu den Niederdruckproben auf und liegen deutlich oberhalb der jeweiligen Phasengrenze. Für alle Niederdruckproben gilt, dass sie im Phasendiagramm unterhalb der jeweiligen Phasengrenze liegen (Erklärung siehe oben). Für die Hochdruckproben liegen alle Werte oberhalb der entsprechenden Phasengrenze. Somit kann von einer guten Unterscheidbarkeit der Hoch- und Niederdruckproben ausgegangen werden.

Der nicht-statistische Aufbau für Niederdruckproben ist schematisch in Abbildung 5.2 gezeigt. Für Niederdruckproben ergibt sich eine höhere Anzahl an benachbarten Säuregruppen. ${ }^{[5]}$ Das wurde ebenfalls in Kapitel 4.13.2 beim direkten Vergleich mit den jeweiligen Hochdruckproben gefunden. 
a)

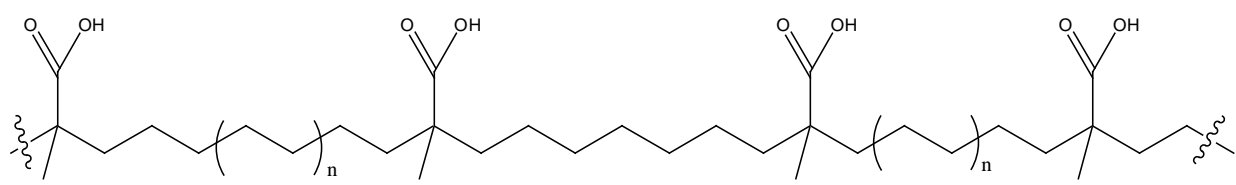

b)

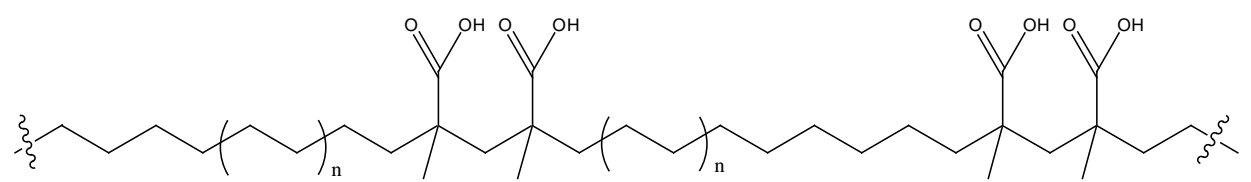

Abbildung 5.2: Schematische Darstellung von statistisch (a)) und nicht-statistisch (b)) aufgebauten EMAA-Copolymeren, $n$ steht für eine unbestimmte Anzahl an Ethyleneinheiten.

Der nicht-statistische Aufbau der Niederdruckproben wird nun zur Erklärung der in Kapitel 4 gemachten Beobachtungen herangezogen. Durch die höhere Anzahl an Säuredimeren (oder höhere Säureoligomere) ergeben sich neue Relaxationsmöglichkeiten für das Copolymer. Es kann aufgrund der Säuregruppen zu einer Versteifung zum Beispiel an den Kettenenden oder zur verstärkten Ausbildung von intramolekularen Wasserstoffbrückenbindungen (WBB) kommen, wie Abbildung 5.3 zeigt. Das führt $\mathrm{zu}$ einer Verkürzung der relaxierenden Elemente. Da nun weniger Energie für diese Segmentbewegung bereitgestellt werden muss, kommt es in DSC-Experimenten zu einer Aktivierung bei tieferen Temperaturen. Das könnte sich durch ein zusätzliches Signal äußern. In der DSC-Analyse in Kapitel 4.10 zeigt sich ein zusätzliches Signal bei tieferen Temperaturen als die $\alpha$-Relaxation (Glasübergang) für die Niederdruckproben, welches bei den Hochdruckproben bei tiefen Synthesetemperaturen nicht und bei hohen Synthesetemperaturen deutlich schwächer als bei den Niederdruckproben beobachtet wird.

Bei einer stationär höheren Konzentration an Säuregruppen kann es verstärkt zur Ausbildung von intramolekularen Wasserstoffbrückenbindungen (WBB) kommen. Diese WBB schränken ebenfalls die resultierende Relaxation ein und können mit einer Verzweigung oder Vernetzung verglichen werden. Die Erhöhung des Vernetzungsgrades führt zu einer Behinderung der $\alpha$-Relaxation. ${ }^{[7]}$ Eine eventuelle zusätzliche Relaxation ist zu tieferen Temperaturen verschoben, was auch in Kapitel 4.10 und 4.11 beobachtet wurde. Als Erklärungsansatz für eine verstärkte Ausbildung von WBB kann herangezogen werden, dass mehrere polare Bereiche entlang einer Polymerkette entstehen können und diese bevorzugt zu anderen polaren Bereichen WBB ausbilden können. Somit kann es in bestimmten Bereichen des Polymers zu einer Vielzahl von WBB führen, die eine erheblich Versteifung (aufgrund ihrer lokal hohen Anzahl) in das Polymer einbringen. Für isolierte 
Säuregruppen ist eine Ausbildung von WBB nur möglich, wenn die Struktur der Polymere eine räumliche Nähe der Säuregruppen zulässt. Über das Polymer zufällig verteilte WBB führen nicht zu einer derartigen Versteifung, wie sie durch mehrere lokal begrenzte WBB gegeben ist.

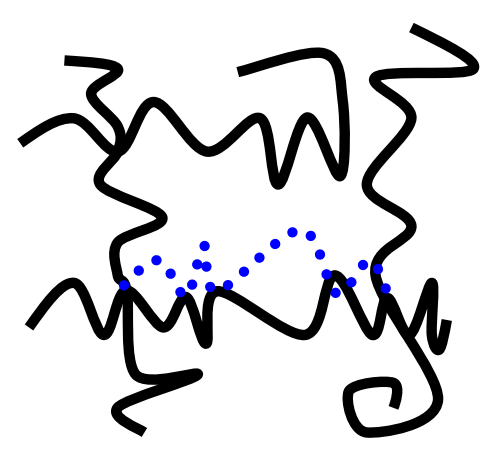

a)

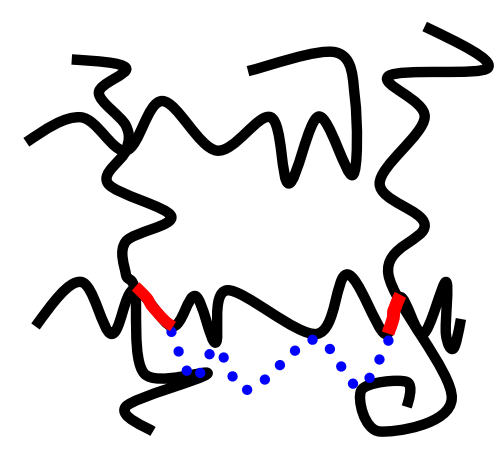

b)

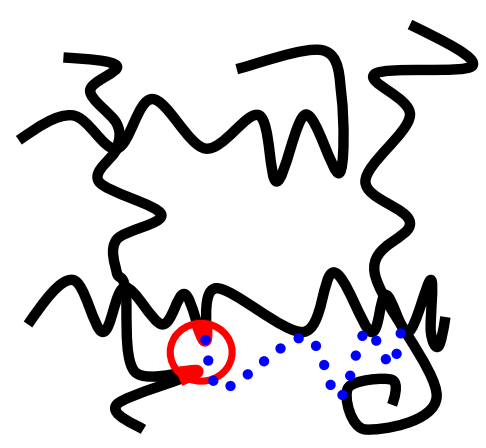

c)

Abbildung 5.3: Schematische Darstellung eines statistisch aufgebauten Copolymers (a)) und einer möglichen Relaxation (blau), eines nicht-statistisch aufgebauten Copolymers (b)) mit einer Anhäufung an Säuregruppen, die die Polymerkette versteifen können (rot) und eines nicht-statistisch aufgebauten Copolymers (c)), wo eine Anhäufung von Säuregruppen zur Ausbildung intramolekularer Wasserstoffbrückenbindungen führen kann (roter Kreis).

Da es sich bei dem in dieser Arbeit gefundenen Effekt ebenfalls um eine Behinderung der Segmentbewegung handelt, die ihren Ursprung in der chemischen Natur des Copolymers hat, wird dieses Verhalten als chemische Behinderung bzw. als „Chemical Confinement“ bezeichnet. ${ }^{[2]}$

\section{2 Überprüfung der Theorie des „Chemical Conefinements“ anhand der erzielten Analyseergebnissen}

Im Folgenden wird die Theorie des „Chemical Confinements" (CC) anhand aussagekräftiger Analysemethoden aus Kapitel 4 überprüft. Als besonders geeignet erweisen sich DSC, DMA und DES-Analysen. Der nicht-statistische Aufbau der 
Niederdruckproben, der dem CC zugrunde liegt, wurde mittels mehrdimensionaler NMRMessungen bereits in Kapitel 4.13.2 gezeigt. In den einzelnen Kapiteln wurde bereits auf die Unterscheidung zwischen Hoch- und Niederdruckproben detailliert eingegangen. In diesem Abschnitt werden die gemachten Beobachtungen mit dem CC verknüpft.

\subsubsection{DSC-Analysen: Chemical Confinement in Niederdruckproben}

Die Beeinträchtigung der Segmentbewegung durch das CC führt, wie oben bereits beschrieben zur Verkürzung der relaxierenden Kette. Durch diesen Effekt kommt es zu einem zusätzlichen Übergang, der bei den Niederdruckproben beobachtet werden kann. Von entscheidendem Einfluss auf die Intensität des in Kapitel 4.10.1 beschriebenen $\beta$-Übergangs ist der MAA-Gehalt im Copolymer. Abbildung 4.27 zeigt die Probenreihe mit dem niedrigsten MAA-Gehalt im Copolymer. Es ist kein signifikanter Unterschied zwischen der Hoch- und Niederdruckprobe zu beobachten. Abbildung 4.25 und 4.26 zeigen Probenreihen mit einem jeweils höherem MAA-Gehalt (siehe Tabelle 4.1).

Beim Vergleich dieser Auftragungen ist eine deutliche Verstärkung der $\beta$-Relaxation mit steigendem MAA-Gehalt im Copolymer zu erkennen. Dies spricht für die Gültigkeit des CC. Je mehr Säureeinheiten vorhanden sind, desto häufiger kann es zu einer Versteifung oder einer Verhakung der Polymerketten kommen. Die DSC-Messungen bestätigen auch den nicht-statistischen Aufbau der Niederdruckproben. Sollte der zusätzliche Übergang lediglich auf das Vorhandensein einer großen Anzahl von Säureeinheiten im Copolymer zurückzuführen sein, dann sollten auch die Hochdruckproben einen weiteren Übergang zeigen, speziell die Probe EMAA 3.4, die einen relativ hohen Säuregehalt besitzt. Für diese Hochdruckprobe wird aber kein signifikanter Übergang beobachtet. Somit wird der unterschiedlich statistische Aufbau der Hoch- und Niederdruckproben ebenfalls bestätigt.

Wie bereits erwähnt, weist die Ausbildung eines $\beta$-Minimums eine Temperaturabhängigkeit auf, wie in Abbildung 4.30 gezeigt. Bei hohen Synthesetemperaturen kommt es zu einer deutlicheren Ausprägung des $\beta$-Übergangs. Die Unterschiede, die sich in den DSC-Messungen für Hoch- und Niederdruckproben ergeben, werden auf den statistisch und nicht-statistischen Aufbau der Copolymere bezüglich der Säuregruppen zurückgeführt, wie es in der Literatur ${ }^{[8,9]}$ und in dieser Arbeit in Kapitel 4.13.2 mittels NMR-Analysen aufgezeigt wird. Durch den nicht-statistischen Aufbau der Niederdruckproben besteht die Möglichkeit, das Konzept des „Chemical Confinements“ zur Erklärung eines zusätzlichen 
$\beta$-Übergangs heranzuziehen. Unter Berücksichtigung des literaturbekannten Phasendiagramms für EMAA-Copolymere, gezeigt in Abbildung 5.1, ergibt sich, dass eine höhere Synthesetemperatur bei gleichem Reaktionsdruck zu einer weiteren Entfernung von der Phasengrenze führt. Somit sollte der nicht-statistische Aufbau zurück gehen und sich das Verhalten der Proben dem Verhalten der statistisch aufgebauten Copolymere annähern. Die Temperaturabhängigkeit der Intensität des $\beta$-Minimums zeigt allerdings einen entgegengerichteten Trend auf. Für hohe Synthesetemperaturen ergibt sich ein intensiver $\beta$-Übergang. Dies könnte bedeuten, dass bei hohen Temperaturen nicht-statistische Proben erhalten werden, was sich allerdings nicht mit der Literatur und den NMR-Ergebnissen aus Kapitel 4.13.2 deckt. ${ }^{[8,9]}$ Beim direkten Vergleich von Hoch- und Niederdruckproben wird ein Einfluss des Synthesedrucks beobachtet. Je näher dieser Synthesedruck an der Phasengrenzen liegt, desto deutlicher werden zusätzlich Übergänge ausgeprägt. Das lässt sich plausibel durch einen nicht-statistischen Aufbau nahe der Phasengrenze erklären. Für die Temperaturabhängigkeit der Intensität des $\beta$-Minimums müssen weitere Effekte von Bedeutung sein. Wie bei der Definition des CC in Kapitel 5.1 erwähnt, führen zusätzlich in das Polymer eingebrachte Verzweigungen, die mögliche Verhakungspunkte bieten, ebenfalls zu einem Einfluss auf mögliche Relaxationen. Dieses „Geometric Confinement“ hängt somit vom Verzweigungsgrad der Polymere ab. Aus der Literatur ist bekannt, dass der Verzweigungsgrad von der Synthesetemperatur und vom Gesamtmonomerumsatz abhängig ist. ${ }^{[10,11]}$ Der Verzweigungsgrad ist umso höher, je höher die Synthesetemperatur und der Umsatz einer Polymerisation sind. Bei hohen Synthesetemperaturen kommt es zu einer verstärkten Bildung von Verzweigungen. Diese Verzweigungen führen, wie auch das $\mathrm{CC}$, zu einer Verkürzung der beweglichen Ketten (siehe Abbildung 5.3). Die stärkere Ausbildung eines $\beta$-Übergangs bei höheren Synthesetemperaturen kann somit plausibel erklärt werden. Bei Betrachtung des in Tabelle 4.2 aufgeführten Umsatzes für die jeweiligen Proben fällt auf, dass für Proben, die bei einer niedrigeren Temperatur synthetisiert wurden, ein geringerer Umsatz vorhanden ist $\left(T_{\text {Reak }}=230{ }^{\circ} \mathrm{C}, X=9.8 \%\right)$, wie für Proben, die bei hohen Temperaturen hergestellt wurden $\left(T_{\text {Reak }}=300{ }^{\circ} \mathrm{C}, X=20 \%\right)$. Die Ausbildung des $\beta$-Übergangs wird bei hohen Synthesetemperaturen durch die Abhängigkeit des Verzweigungsgrades von der Temperatur und vom Umsatz verstärkt. Für die Proben, die bei hohen Temperaturen hergestellt wurden, summieren sich somit beide Effekte, da sowohl eine höher Synthesetemperatur und auch ein höherer Umsatz vorliegen als für Proben, die bei einer niedrigen Temperatur synthetisiert wurden. Ein Einfluss des Synthesedrucks auf die Verzweigungen wird aufgrund der bekannten Aktivierungsvolu- 
men für zum Beispiel die Homopolymerisation von Ethen $\left(-3.4 \mathrm{~cm}^{3} \cdot \mathrm{mol}^{-1}\right)^{[12]}$ als gering eingeschätzt, kann aber nicht gänzlich ausgeschlossen werden. Eine Erklärung der beobachteten starken Druckabhängigkeit lediglich mittels Verzweigungen ohne Berücksichtigung des $\mathrm{CC}$ wäre theoretisch möglich, erscheint aber nicht realistisch. Als Gründe können aufgeführt werden, dass die oben beschriebene Druckabhängigkeit bei Weitem nicht ausreichen würde, um die erheblichen Unterschiede der vermessenen Hochund Niederdruckproben einer Probenreihe mit nahezu gleichem Umsatz zu erklären. Als Beispiel kann die Probenreihe EMAA 3 aufgeführt werden. Ein erheblicher Einfluss des Synthesedruckes auf das resultierende Thermogramm der vermessenen Proben kann auch bei der Probenreihe EMAA 28 festgestellt werden.

Ein Einfluss des nicht-statistischen Aufbaus der Polymere und somit eine Auswirkung des CC kann beim direkten Vergleich der in Abbildung 5.4 gezeigten Proben beobachtet werden.

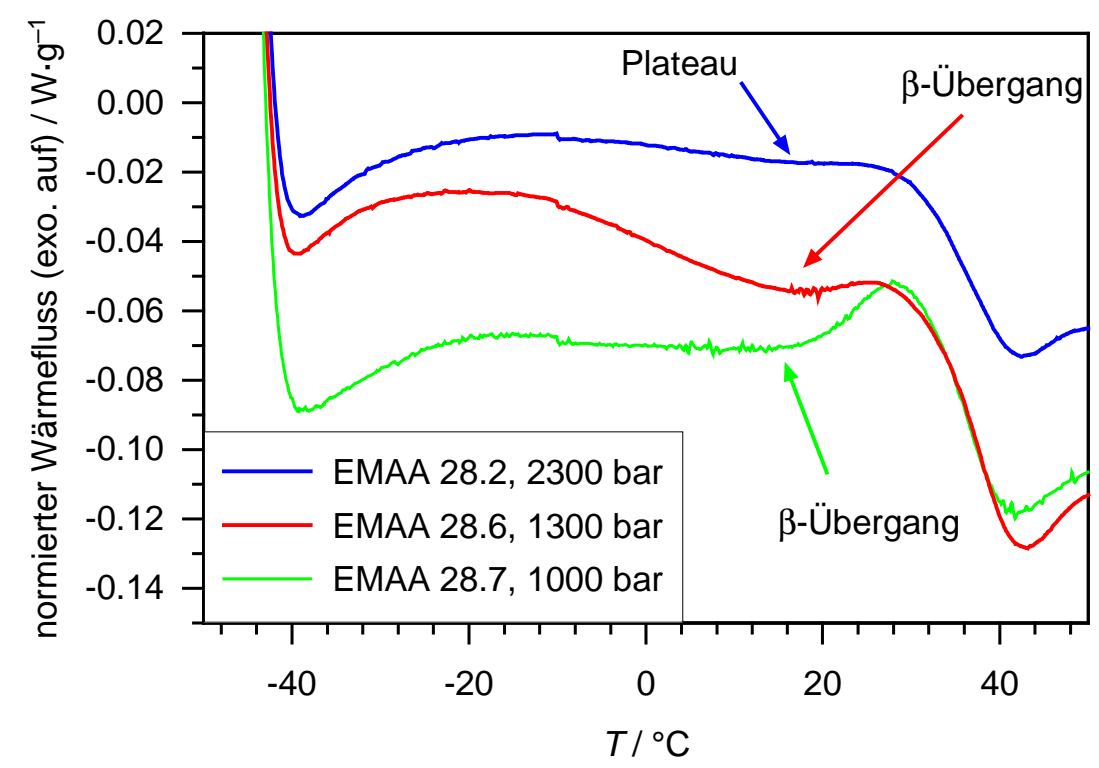

Abbildung 5.4: DSC-Thermogramm für EMAA 28.2, 2300 bar, $X=23.6 \%, F_{M A A}=0.035$, EMAA 28.6, 1300 bar, $X=20.5 \%, F_{M A A}=0.036$, EMAA 28.7, $1000 \mathrm{bar}$, $X=20.1 \%, F_{M A A}=0.036$.

Die Hochdruckprobe zeigt eine leichte Plateaubildung im Temperaturbereich, wo ein $\beta$-Übergang für die Niederdruckproben erwartet wird. Es liegt ein Umsatz von $23.6 \%$ vor. Bei diesem Umsatz und der hohen Synthesetemperatur von ca. $300{ }^{\circ} \mathrm{C}$ kommt es vermehrt zur Ausbildung von Verzweigungen im Vergleich $\mathrm{zu}$ Proben, die bei niedrigeren Temperaturen und Umsätzen synthetisiert wurden. Proben, die nahe der Phasengrenze hergestellt wurden und somit einen nicht-statistischen Aufbau aufweisen, zeigen einen 
ausgeprägten $\beta$-Übergang (siehe auch Abbildung 4.30). Der Vergleich der beiden Niederdruckproben, die bei 1000 und 1300 bar bei nahezu gleichen Temperaturen und gleichen Umsätzen hergestellt wurden, lässt vermuten, dass die Ausbildung des $\beta$-Übergangs sowohl auf Verzweigungen als auch auf das CC zurückzuführen sind. Die bei 1000 bar nahe der Phasengrenze hergestellte Probe zeigt einen intensiveren Übergang als die bei 1300 bar hergestellte Probe, die aufgrund der Entfernung zur Phasengrenzen einen weniger nicht-statistsichen Aufbau aufweisen sollte.

Nun lässt sich auch die Aufspaltung des $\beta$-Übergangs in Abbildung 4.26 für die Niederdruckprobe EMAA 3.12 erklären. Diese Probe wurde bei einem Umsatz von $23.6 \%$, einer Temperatur von $T_{\text {Reak }}=270{ }^{\circ} \mathrm{C}$ und einem MAA-Gehalt von $F_{\text {MAA }}=0.066$ erhalten. Es liegen Bedingungen vor, die einen hohen Verzweigungsgrad erwarten lassen. Des Weiteren ist bei diesem relativ hohem MAA-Gehalt und einem nicht-statistischen Aufbau das CC stark begünstigt. Durch die möglichen Verzweigungen kann es zu einem „Geometric Confinement“ kommen, das durch das CC weiter verstärkt wird. Es ist nicht ausgeschlossen, dass dieser Effekt zu einer Separation des $\beta$-Übergangs führt. Für die in Abbildung 5.4 gezeigten Proben liegt im Vergleich zu EMAA 3.12 nur ca. ein halb so hoher Säuregehalt im Copolymer vor. Dadurch ist es möglich, dass das CC aufgrund des geringeren Säuregehaltes zu einer Verbreiterung führt, aber keine Aufspaltung wie im Falle von EMAA 3.12 herbeiführen kann.

\subsubsection{Chemical Confinement bei der mechanischen Spektroskopie}

Bei den DMA-Analysen wurde ein zusätzlicher Übergang für die untersuchten Niederdruckproben gefunden. Bei Hochdruckproben ließ sich keine $\beta$-Relaxation beobachten. Auch diese Analysen weisen auf das Vorhandensein des CC hin. Besonders interessant ist die starke Kräfteabhängigkeit der $\beta$-Relaxation, gezeigt in Kapitel 4.11.3. Während die dort erwähnte $\gamma$ - und $\alpha$-Relaxation keine starke Kräfteabhängigkeit zeigen, verschiebt sich die $\beta$-Relaxation bei Erhöhung der angelegten Kräfte sehr stark zu niedrigeren Temperaturen. Ein Erklärungsansatz beruht auf der hohen mechanischen Belastung des Copolymers. Durch die Komprimierung können eventuell zusätzliche WBB ausgebildet werden. Das beruht auf einer Distanzverkürzung zwischen den Polymerketten. Somit ist es für säurereiche Regionen wahrscheinlicher, dass WBB zu ebenfalls säurereichen Sequenzen einer weiteren Seitenkette ausgebildet werden. Wie in 
Abbildung 5.3 c) gezeigt, kann dies zu einer Verkürzung der relaxierenden Segmente führen. Die starke Kräfteabhängigkeit der $\beta$-Relaxation und der nicht vorhandene Einfluss auf die restlichen Relaxationen spricht ebenfalls für ein CC.

\subsubsection{Chemical Confinement in der dielektrischen Spektroskopie}

Mit Hilfe der DES-Messungen konnten die gleichen Tendenzen wie bei den DMAUntersuchungen aufgezeigt werden. Auch hier zeigt sich für die Niederdruckproben eine zusätzliche Relaxation. Diese verschiebt sich bei niedrigen Frequenzen stark zu tieferen Temperaturen im Gegensatz zu den weiteren beobachteten Übergängen. Das Auffinden des zusätzlichen Übergangs bestätigt auch hier die Gültigkeit der CC-Theorie.

\section{Literaturverzeichnis Kapitel 5}

[1] J. Hachenberg, Dissertation, Göttingen (2006)

[2] J. Hachenberg, B. Steisel, Undrakh Nergui, D. Bedorf, M. Buback, K. Samwer, New Journal of Physics, eingereicht

[3] A. Schönhals, H. Goering, Ch. Schick, J. of Non-Cryst. Sol., 305 (2002) 140

[4] A. Serghei, F. Kremer, Phys. Rev. Lett. 91 (16) (2003) 165702

[5] United States Patent 4,248,990

[6] M. Buback, H. Latz, Macromol. Chem. Phys. 204 (2003) 638-645

[7] S. Kalakkunnath, D. S. Kalika, H. Lin, B. D. Freeman, Macromolecules 38, (2005) 9679

[8] United States Patent 4,248,990

[9] European Patent 0146620B1

[10] B. Steisel, Diplomarbeit, Göttingen (2004)

[11] M. van Boxtel, M. Busch, S. Lehmann, Macromol. Chem. Phys. 201(3), (2000), 313

[12] M. van Boxtel, Dissertation, Göttingen (2000) 


\section{Ausblick}

In dieser Arbeit wurden signifikante Unterschiede in den Materialeigenschaften der hergestellten Copolymerproben EMAA festgestellt. Das „Chemical Confinement“ wird erstmalig für dieses System in Zusammenarbeit mit dem Arbeitskreis von Prof. Samwer definiert. ${ }^{[1]}$ Wünschenswert ist ein noch detaillierteres Studium des nicht-statistischen Aufbaus der nahe der Phasengrenzen synthetisierten Copolymerproben.

Eine Unterscheidungsmöglichkeit für säurezentrierte Di- und Triaden stellt die quantitative ${ }^{13}$ C-NMR-Spektroskopie in flüssiger Phase dar, was bereits an Ethen-AcrylatCopolymersystemen erfolgreich durchgeführt werden konnte. ${ }^{[2]}$ Diese Untersuchungen würden zusätzlich einen aussagekräftigen Wert für den Verzweigungsgrad der Copolymere liefern. Anhand dieser Daten und der vorhandenen Molmassenverteilungen ist es möglich, Modellierungen mit dem Programmpaket PREDICI $^{\circledR}$ durchzuführen und Reaktionskoeffizienten für die stattfindenden Übertragungsreaktionen zu bestimmen. ${ }^{[3]}$ Eine weitere Herausforderung stellt die Implementierung des nicht-statistischen Einbaus der Säuremonomere dar.

Als weiteres, interessantes System kann die Verwendung von Acrylsäure ${ }^{[4]}$ als Comonomer die in dieser Arbeit gemachten Beobachtungen bestätigen und Aufschlüsse über den Einfluss der zusätzlichen bei der Methacrylsäure vorhandenen Methylgruppe geben. Gerade im Hinblick auf die mechanische Spektroskopie ist es erstrebenswert, den Einfluss der Methylgruppe auf die beobachteten Relaxationen zu quantifizieren.

Höchst interessant, wenn auch aparativ bedingt äußerst schwierig, erscheint eine Terpolymerisation von Ethen, Methacrylsäure und Acrylsäure. Durch geeignete Analyseverfahren kann eventuell eine verstärkte Bildung von Säuregruppen einer Art, oder eventuell auch eine nicht-statistische Anhäufung von Methacrylsäure/Acrylsäure-Dimeren, gefunden werden. Diese Idee ließe sich auch auf Acrylate erweitern, sodass Ethenterpolymere entstehen, die sowohl Methacrylsäure als auch Methylmethacrylat enthalten.

Unter Verwendung eines weiteren Rührkessels könnte eine Eindosierung des Acrylats in einen separaten Reaktionsraum, von der Säuredosierung getrennt, vorgenommen werden. Hierfür müsste allerdings eine Pumpe zur Verfügung stehen, die es vermag, bei hohen Reaktionsdrücken niedrige Volumenströme mit höchster Präzision über einen ausreichend langen Zeitraum direkt in den Reaktor zu dosieren. 
Wie bereits in Kapitel 4.14.7 erwähnt, wäre eine weitere Aufklärung des PLDMechanismus von Vorteil. Hierfür würde sich sehr gut ein Copolymersystem eignen, in welchem ein weiter Bereich an enthaltenem polaren Monomer synthetisiert werden kann. Ebenfalls ist die Verwendung von engverteiltem Polymer ratsam. Kontrollierte radikalische Polymerisation stellt hier eine Option dar.

Weitere Untersuchungen der Materialeigenschaften sind anzuraten. So können zum Beispiel rheologische Untersuchungen helfen, zwischen statistisch und nicht-statistisch aufgebauten Copolymeren $\mathrm{zu}$ differenzieren. Vielversprechende rheologische Untersuchungen an Polyethylen konnten bereits von Becker durchgeführt werden. ${ }^{[5]}$

\section{Literaturverzeichnis Kapitel 6}

[1] J. Hachenberg, B. Steisel, Undrakh Nergui, D. Bedorf, M. Buback, K. Samwer, New Journal of Physics, eingereicht

[2] M. van Boxtel, Dissertation, Göttingen (2000)

[3] M. Buback, M. Busch, R. A. Lämmel, Mocromol. Theory Simul. 5 (1996) 1652

[4] U. Nergui, Dissertation, Göttingen (2008)

[5] F. Becker, Dissertation, Göttingen (2006) 


\section{Anhang}

\subsection{Abkürzungsverzeichnis}

\begin{tabular}{|c|c|}
\hline Abkürzung/Zeichen & Bedeutung \\
\hline AA & Acrylsäure \\
\hline ATR & abgeschwächte Totalreflektion \\
\hline $\mathrm{CC}$ & "Chemical Confinement" \\
\hline $\mathrm{CP}$ & "Cross Polarization" \\
\hline d & dynamische Kraft \\
\hline DES & Dielektrische Spektroskopie \\
\hline DMA & dynamisch-mechanische Analyse \\
\hline DSC & „Differential Scanning Calorimetry“ \\
\hline E & elektrisches Feld \\
\hline $\mathrm{E}$ & Ethen \\
\hline EAA & „Poly(ethene-co-acrylic acid)" \\
\hline EMAA & „Poly(ethene-co-methacrylic acid)" \\
\hline$f$ & Monomergehalt im Dosierstrom \\
\hline$F$ & Monomergehalt im Copolymer \\
\hline FT & fouriertransformiert \\
\hline GPC & Gelpermeationschromatographie \\
\hline HETCOR & heteronukleare Korrelationsspektroskopie \\
\hline HK & Hauptkette \\
\hline HPLC & "High performance liquid chromatographie" \\
\hline Ini & Initiatorfluss \\
\hline IR & infrarot \\
\hline $\lg$ & $\log _{10}(\mathrm{Zahl})$ \\
\hline$M$ & Molmasse \\
\hline MAA & Methacrylsäure \\
\hline MAPLE & "Matrix Assisted Pulsed Laser Evaporation" \\
\hline MAS & "Magic Angel Spinning" \\
\hline MMA & Methylmethacrylat \\
\hline$M_{\mathrm{n}}$ & Zahlenmittel der Molekulargewichtsverteilung \\
\hline
\end{tabular}




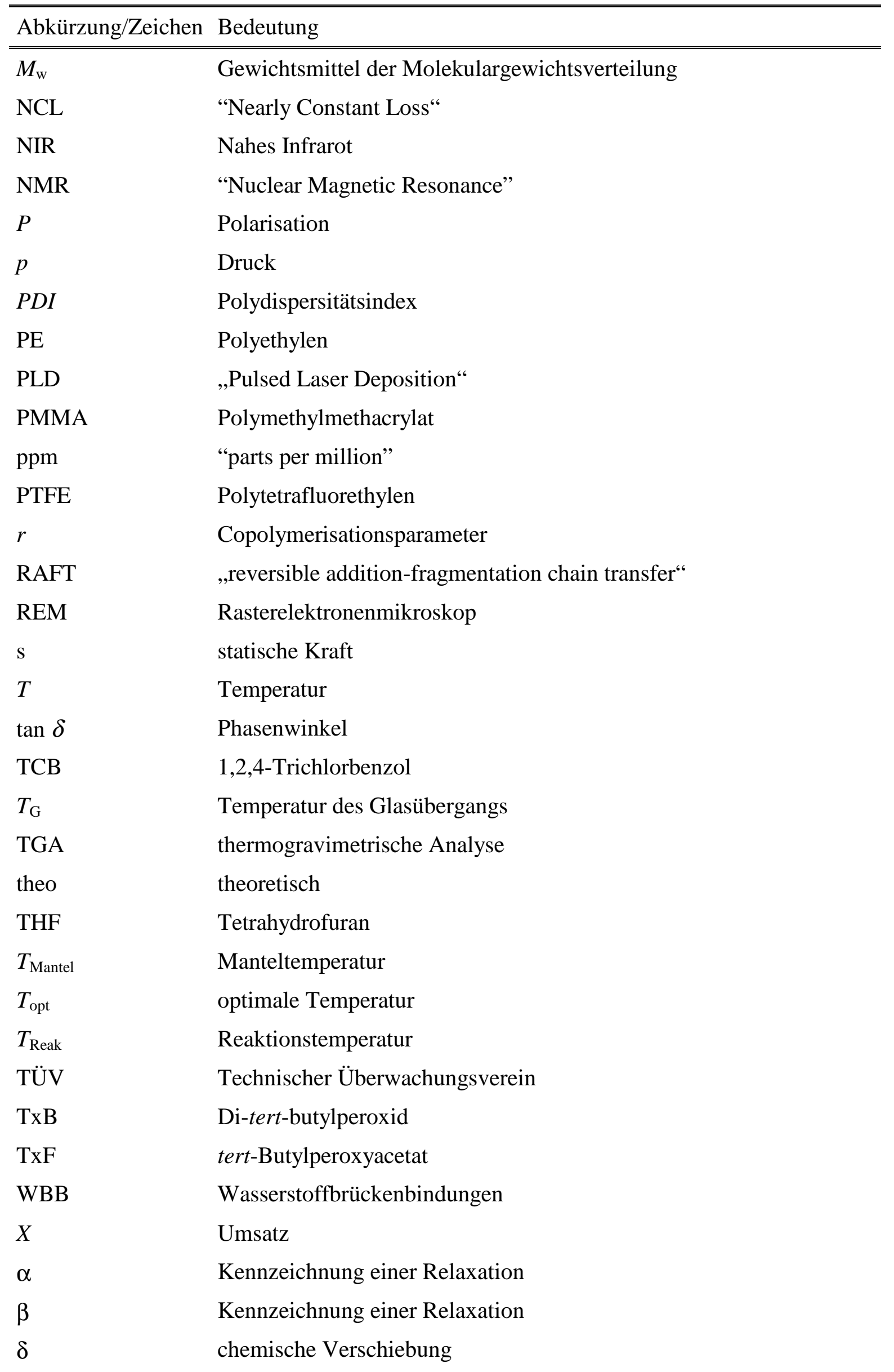




\begin{tabular}{ll}
\hline \hline Abkürzung/Zeichen & Bedeutung \\
\hline \hline$\varepsilon "$ & dielektrischer Verlust \\
$\gamma$ & Kennzeichnung einer Relaxation \\
$\omega_{1}$ & Resonanzfrequenz der ersten (indirekten) Dimension \\
$\omega_{2}$ & Resonanzfrequenz der zweiten (direkten) Dimension \\
\hline
\end{tabular}




\subsection{TÜV-Überprüfung der Ethenreinigungstürme}

Die Druckbehälter zur Reinigung des Ethens im Minitechnikum 1 müssen alle 5 Jahre einer Sichtprüfung durch den TÜV unterzogen werden. Alle 10 Jahre steht eine Druckprüfung an. Die nächste Prüfung ist für das Jahr 2010 vorgesehen. Dann muss sowohl eine Sichtprüfung als auch eine Druckprüfung durchgeführt werden.

Die letzte Prüfung erfolgte im Dezember 2005. Im Folgenden wird eine detaillierte Aufstellung der Vorgehensweise wiedergegeben, welche für zukünftige Prüfungen eine Hilfe bereitstellen soll.

\subsubsection{Allgemeine Vorbereitungen}

Es empfiehlt sich frühzeitig einen Messplan zu erstellen und die Ausfallzeiten des MiniTechnikums für eventuell anstehende Reparaturen zu nutzen. Des Weiteren sollte im Vorfeld unter Rücksprache mit der Werkstatt des IPC ein TÜV-Termin vereinbart werden. Da der Katalysator im reduzierten Zustand pyrophor ist, muss er vor Öffnen des Druckbehälters in die oxidierte Form überführt werden $(\mathrm{CuO})$. Deswegen ist sicherzustellen, dass ausreichend Gas für die Deaktivierung vorhanden ist (siehe auch Kapitel 7.2.2).

\subsubsection{Deaktivierung des Katalysators}

Die Anleitung der BASF AG zum Umgang mit dem Katalysator ist zu beachten.

Alle $\mathrm{Zu}$ - und Abgänge des Katalysatorturms schließen. Vor dem Zugangsventil ein Adapterstück zum Schlauchanschluss installieren. Gasflaschen nach Möglichkeit in sicherer Entfernung zum Katalysatorturm positionieren (andere Laborhälfte). Direkt nach den Drosselventilen der Gasflaschen einen Durchflussmesser (Blasenzähler) installieren um den Gasstrom und somit die Zusammensetzung des Gasgemisches verfolgen zu können. Das Gemisch sollte zu ca. $1 \%$ aus Sauerstoff $\left(\mathrm{O}_{2}\right.$, technisch) und zum überwiegenden Teil aus Stickstoff $\left(\mathrm{N}_{2}\right.$, Reinheit: 4.6 oder 5.5) bestehen. Von der Verwendung von technischem Stickstoff ist zu Beginn der Reaktion abzuraten, da bereits die Spuren von Sauerstoff, die in technischem Stickstoff enthalten sein können, ausreichen, um die Reaktion unkontrollierbar voran zu treiben. Zum Ende der Reaktion ist der Einsatz von technischem Stickstoff nicht mehr so riskant, allerdings trotzdem nicht 
empfehlenswert. Der Gasstrom wird nach jeweils zwei Meter mittels eines T-Stückes vereint und dann einsträngig über eine Distanz von ca. fünf Metern zum Katalysatorturm geleitet. Dies dient zur optimalen Durchmischung des Gasgemisches. Während des Betriebs ist darauf zu achten, dass kein Silikonöl aus den Blasenzählern in den Katalysatorturm gelangt. Es ist eine Austrittsöffnung für den Gasstrom zu schaffen. Dies lässt sich am besten durch ein kurzes Verbindungsstück zur internen Absaugvorrichtung realisieren.

Der Katalysatorturm verfügt über ein Heiz- und ein Kühlsystem. Die Oxidation des Katalysators kann bei Raumtemperatur durchgeführt werden. Es ist jedoch dringend anzuraten, einen Kühlkreislauf aufzubauen, um notfalls schnell ins Reaktionsgeschehen einzugreifen zu können und die Reaktion gegebenenfalls abzukühlen.

Der Katalysatorturm besitzt vier Anschlüsse für eine Temperaturkontrolle. Drei Anschlüsse befinden sich am Turmkopf und ein Anschluss seitlich. Der seitlich angebrachte Anschluss dient zur Temperaturkontrolle der Heizung. Bei den oben angebrachten Termosteckern handelt es sich um Innenthermoelemente. Diese Thermoelemente befinden sich an drei unterschiedlichen Stellen im Inneren des Turms. Der Aufbau ist analog zur Anbringung der Thermostecker, das bedeutet, dass der Stecker in der oberen Position dem obersten Innenthermoelement zuzuordnen ist. Beim Anschluss von Manometern ist darauf $\mathrm{zu}$ achten, dass die Polung stimmt. Die Polarität aller Thermoelemente, die in den Türmen verbaut wurden steht der üblich verwendeten Polarität im Minitechnikum entgegen. Deswegen empfiehlt sich der Einsatz von gekreuzten Thermoelementleitungen. Zeigen alle drei Innenthermoelemente sinnvolle Werte und wurde die Zuleitung des Gasgemisches sowie der Kühlkreislauf auf Dichtigkeit geprüft, sollte das restliche sich unter Druck im Turm befindende Ethen abgelassen werden. Nun kann durch Öffnen der entsprechenden Ventile mit der Oxidation begonnen werden. Es ist darauf zu achten, dass Temperaturen von über $250{ }^{\circ} \mathrm{C}$ unter allen Umständen vermieden werden müssen, da es sonst zu einer unkontrollierbaren Reaktion und zur Zerstörung des Katalysators kommen kann. Verbrannter Katalysator, der durch seine rosa-kupferne Farbe und durch Zusammenklumpen der Katalysatorpalletts $\mathrm{zu}$ erkennen ist, kann nicht wiederverwendet werden. Das Starten der Reaktion äußert sich durch einen langsamen Anstieg der Temperatur im oberen Bereich des Katalysatorturms. Der Sauerstoffgehalt des Gasgemisches darf nur langsam (unter Umständen gar nicht) erhöht werden und es ist auf den Temperaturanstieg, der stark zeitversetzt eintreten kann, zu achten.

Bei einer Deaktivierung ist je nach Verlauf mit einer Zeit von bis zu drei Tagen zu rechnen. Sollte die Temperatur zu rasant ansteigen, ist zuerst der Sauerstoffgehalt des 
Gemisches zu reduzieren oder die Sauerstoffzufuhr komplett zu unterbinden. Sollte auch diese Maßnahme keine Wirkung zeigen, so ist der Stickstoffstrom zu stoppen und gegebenenfalls die Kühlung einzuschalten. Die Gasdosierung darf erst wieder erfolgen, wenn in allen Bereichen des Turms eine deutlich unterhalb von $250^{\circ} \mathrm{C}$ liegende Temperatur stabil vorliegt.

Während der Oxidation beobachtet man das Vorranschreiten der Reaktionszone anhand des Temperaturverlaufes. Wenn die Temperatur in der untersten Zone des Turms nach Erreichen eines Maximums (Temperaturmaximum ist abhängig vom Sauerstoffgehalt der Gasmischung) kontinuierlich abfällt, ist der Sauerstoffgehalt des Dosiergases vorsichtig unter Berücksichtigung der Temperatur zu erhöhen. Die Reaktion ist beendet, wenn bei Eindosierung von reinem Sauerstoff über einen längeren Zeitraum keine Temperaturerhöhung mehr zu beobachten ist.

Der Turm ist nun mit Stickstoff zu spülen, unter Berücksichtigung eines Innenvolumens von ca. 11 Litern.

Alle Kapillaren und Anschlüsse, die nicht mehr benötigt werden, sind erst nach Abkühlung des Turms zu entfernen.

Der Katalysator liegt nun in der oxidierten Form vor und ist unter Vorsicht an Umgebungsluft handhabbar.

\subsubsection{Abbau der Türme}

Zum Entfernen des Molsiebs bedarf es keiner vorherigen Deaktivierung. Alle Zu- und Abgangskapillaren sind zu entfernen. Der Behälter kann unter Normalbedingungen geöffnet werden.

Aufgrund des Gewichtes und der Platzsituation im Technikum ist es empfehlenswert, die Türme mit 2 Personen aus den Halterungen zu heben. Es bedarf allerdings weiterer Vorbereitung. Zwei Schrauben, die den oberen Flansch fixieren, sind zu entfernen. In die nun freien Bohrungen sind zwei Schekel einzubringen, durch die eine ausreichend stabile Stange geführt wird. Mit Hilfe dieser Stange ist nun eine Entnahme der Türme möglich.

Für das weitere Vorgehen ist es sinnvoll, die Türme in das Werkstattgebäude zu bringen. Dort kann man aufgrund der besseren Platzsituation die Türme bequem öffnen. Es sind ausreichend Behälter für die Molsieb- und die Katalysatorperlen bereitzustellen, da beide Materialien wiederverwendet werden. Die Türme sind jeweils auf der unteren Seite 
(Boden) zu öffnen, da es so nicht zu Problemen mit den eingearbeiteten Thermoelementen kommt. Das entfernte Molsiebgranulat ist schnellstmöglich luftdicht zu verschließen, um eine erhöhte Wasseraufnahme $\mathrm{zu}$ vermeiden. Beim Öffnen des Katalysatorturms ist weiterhin Vorsicht geboten, da nicht vollständig ausgeschlossen werden kann, dass ein Teil des Kupferkatalysators noch reduziert vorliegt. Bei der Entfernung kommt es zu einer starken Staubentwicklung. Das Einatmen dieses Staubes führt unmittelbar zu Reizungen der Atemwege. Aus diesem Grund sollte eine Kontamination oder sogar eine Inkorporation vermieden werden. Die schwarzen Katalysatorpalletts werden aufgefangen und darauf überprüft, ob sich zerstörte Katalysatorteile finden lassen (siehe Kapitel 7.2.2).

Die Türme werden nun einer Vorabüberprüfung durch die Werkstatt unterzogen und anschließend von einem TÜV-Beauftragten abgenommen.

\subsubsection{Wiederbefüllung und Reinstallation der Reinigungstürme}

Zum Befüllen der Türme erwies es sich als vorteilhaft einen Kran zu verwenden. Die Türme wurden mit dem Boden aufwärts (Schekel und Stange) aufgehangen und wieder befüllt. Zum Verschließen der Turmunterseiten wurden die Türme mit der Oberseite auf zwei Holzblöcke gestellt, um die Thermoelemente und andere Anschlüsse nicht zu beschädigen. Die 12 Schrauben des Bodenflansches wurden kreuzweise mit einem Drehmoment von $35 \mathrm{Nm}$ angezogen. Für die Installation wurde diesmal der vorhandene Kran genutzt. Die Verwendung gestaltete sich allerdings schwierig und benötigte einen großen Zeitaufwand. Zu einer schonenderen Behandlung der Türme konnte diese Methode auch nur bedingt beitragen. Der Kraneinsatz ist also für die Zukunft nicht besonders empfehlenswert. Nach Befestigung der Halteschellen sind auf der Oberseite der Türme die fehlenden Schrauben wieder einzusetzen und alle Schrauben mit einem Drehmoment von $35 \mathrm{Nm}$ anzuziehen.

\subsubsection{Reduktion des Katalysators}

Zur Aktivierung des Katalysators muss dieser in die reduzierte Form überführt werden. Dazu befestigt man alle notwendigen Kapillaren und Ventile (siehe Kapitel 7.2.2). Anstelle von Sauerstoff wird nun ein Reduktionsmittel verwendet. Wasserstoff (3.0) ist hierfür gut 
geeignet. Aufgrund der Wasserbildung wird der Abgasschlauch nicht in die Abluft geführt, sondern direkt ins Freie.

Nachdem alle Temperaturanzeigen installiert worden sind, kann mit dem Aufheizen des Katalysatorturms auf $150{ }^{\circ} \mathrm{C}$ begonnen werden. Hierzu wurden die beiden Heizkreisläufe in Reihe geschaltet und über einen Regler mit Strom versorgt. Es ist auf die richtige Polung zu achten. Dies kann durch eine vorherige Widerstandsmessung gewährleistet werden. Liegt eine homogene Temperaturverteilung im Turm vor, so kann mit der Reduktion begonnen werden.

Zunächst wird reiner Stickstoff durch den Turm geleitet um den restlichen Sauerstoff zu verdrängen. Danach wird wenig Wasserstoff zugesetzt (1\%). Der Beginn der Reaktion lässt sich an einem Temperaturanstieg im Turm und an einsetzender Wasserbildung erkennen. Gegebenenfalls sollte der Wasserstoffanteil im Gasgemisch geringfügig erhöht werden. Temperaturen von über $230{ }^{\circ} \mathrm{C}$ sind auf jeden Fall zu vermeiden. Sollte es zu einem rasanten Temperaturanstieg kommen, so wird der Wasserstoffstrom reduziert bzw. gestoppt (wie in Kapitel 7.2.2 für Sauerstoff beschrieben). Das Voranschreiten der Reaktion lässt sich wieder am Temperaturverlauf im Turm feststellen. Wenn kein Wasser mehr entsteht und die Temperatur am Boden des Turms absinkt, wird vorsichtig der Wasserstoffgehalt erhöht, bis der Turm am Ende nur vom reinem Reduktionsmittel durchströmt wird. Der Turm wird abschließend mit reinem Stickstoff (Reinheit 5.5) gespült und unter etwas Überdruck stehen gelassen. Der Vorgang des Reduzierens wird sich in aller Regel über mehrere Tage ziehen. Es hat sich als sehr vorteilhaft erwiesen, die Heizung des Turmes über Nacht in Betrieb zu lassen, unter Berücksichtigung der notwendigen Vorsicht. Von einer unbeaufsichtigten Gasdosierung wird ausdrücklich abgeraten.

\subsubsection{Regenerierung des Molsiebs}

Um die durch die Luftfeuchtigkeit verursachten Wasserspuren des Molsiebs zu entfernen wird der Turm auf ca. $180{ }^{\circ} \mathrm{C}$ aufgeheizt. Entspricht die Innentemperatur weitestgehend der Manteltemperatur so kann mit dem Evakuieren begonnen werden. Anfänglich findet eine Drehschieberpumpe Verwendung, um die größte Menge Wasser zu entfernen. Nach

zwei bis dreitägigem Vakuum von ca. $10^{-1} \mathrm{mbar}$ wird das Vakuum durch eine Turbomolekularpumpe auf $2.5 \cdot 10^{-3}$ mbar verbessert. Das Vakuum wird für den Zeitraum von einer Woche aufrechterhalten. 
Der Turm wird dann mit Stickstoff (Reinheit 5.5) befüllt.

Nachdem alle ursprünglichen Verbindungen wieder hergestellt sind, wird die Anlage mit

Ethen (Reinheit 3.0) befüllt. Die Türme stehen nun wieder zur Reinigung von Ethen bei Hochdruckpolymerisationen zur Verfügung. 


\section{3 Überblick über maßgebliche Parameter der synthetisierten Copolymerproben}

\begin{tabular}{l|ccccccccc} 
Probe & $p$ & $T_{\text {Mantel }}$ & $T_{\text {Reak }}$ & $\mathrm{E}$ & $\mathrm{MAA}$ & $f_{\text {MAA }}$ & $X$ & $F_{\text {MAA }}$ ATR & $F_{\text {MAA }}$ theo \\
& $/$ bar & $/{ }^{\circ} \mathrm{C}$ & $/{ }^{\circ} \mathrm{C}$ & $/ \mathrm{g} \cdot \mathrm{h}^{-1}$ & $/ \mathrm{g} \cdot \mathrm{h}^{-1}$ & $/ 10^{-3}$ & $/ \%$ & $/ 10^{-2}$ & $/ 10^{-2}$ \\
\hline EMAA 1.2 & 2000 & 242 & 255 & 1800 & 34.2 & 6.1 & 15.2 & 2.4 & 2.7 \\
EMAA 2.2 & 1860 & 245 & $271-276$ & 1800 & 34.2 & 6.1 & 16.7 & 2.4 & 2.5 \\
EMAA 2.10 & 1300 & 246 & $268-272$ & 1800 & 34.2 & 6.1 & 13.2 & 2.6 & 3.0 \\
EMAA 3.4 & 2300 & 244 & 272 & 1800 & 116 & 20.5 & 21.9 & 5.4 & 6.9 \\
EMAA 3.12 & 1300 & 245 & $268-271$ & 1800 & 116 & 20.5 & 23.6 & 5.5 & 6.6 \\
EMAA 12.11 & 2300 & 246 & $263-264$ & 1800 & 5.3 & 1.0 & 10.0 & 0.8 & 0.6 \\
EMAA 12.4 & 1300 & 245 & $268-275$ & 1800 & 5.3 & 1.0 & 10.8 & 0.8 & 0.6 \\
EMAA 16.2 & 2300 & 240 & $236-238$ & 1800 & 58 & 10.4 & 5.6 & 7.2 & 8.2 \\
EMAA 16.15 & 1500 & 240 & $238-239$ & 1800 & 58 & 10.4 & 6.9 & 7.8 & 7.4 \\
EMAA 16.5 & 1300 & 240 & $236-238$ & 1800 & 58 & 10.4 & 6.0 & 8.7 & 7.9 \\
EMAA 22.9 & 2300 & 260 & $256-261$ & 1800 & 58 & 10.4 & 7.2 & 6.8 & 7.0 \\
EMAA 22.14 & 1300 & 260 & $258-261$ & 1800 & 58 & 10.4 & 9.3 & 8.0 & 6.1 \\
EMAA 25.2 & 2300 & 220 & $261-268$ & 1800 & 58 & 10.4 & 17.7 & 4.3 & 4.1 \\
EMAA 25.6 & 1300 & 220 & $262-268$ & 1800 & 58 & 10.4 & 12.1 & 5.9 & 5.1 \\
EMAA 27.5 & 2300 & 200 & $236-243$ & 1800 & 58 & 10.4 & 14.0 & 4.8 & 4.9 \\
EMAA 27.8 & 1400 & 200 & $247-250$ & 1800 & 58 & 10.4 & 16.0 & 4.2 & 4.5 \\
EMAA 28.2 & 2300 & 250 & $294-300$ & 1800 & 58 & 10.4 & 23.6 & 2.5 & 3.5 \\
EMAA 28.6 & 1300 & 250 & $293-295$ & 1800 & 58 & 10.4 & 20.5 & 3.0 & 3.7 \\
EMAA 28.7 & 1000 & 250 & $294-300$ & 1800 & 58 & 10.4 & 20.1 & 3.0 & 3.6 \\
EMAA 30.3 & 2300 & 170 & $214-243$ & 1800 & 58 & 10.4 & 9.7 & 6.7 & 6.5 \\
EMAA 31.2 & 2300 & 155 & $215-242$ & 1800 & 58 & 10.4 & 7.6 & 7.4 & 7.5 \\
EMAA 32.2 & 2300 & 210 & $256-258$ & 1800 & 58 & 10.4 & 17.8 & 3.3 & 4.2 \\
EMAA 32.3 & 1300 & 210 & $254-258$ & 1800 & 58 & 10.4 & 10.4 & 6.0 & 6.0 \\
EMAA 33.3 & 2300 & 190 & $230-232$ & 1800 & 58 & 10.4 & 16.9 & 5.4 & 4.3 \\
EMAA 33.8 & 1400 & 190 & $227-237$ & 1800 & 58 & 10.4 & 9.8 & 8.0 & 6.2
\end{tabular}

Tabelle 7.1: $\quad$ Syntheseparameter, Umsatz und Copolymerzusammensetzung der für weitere Auswertungen benutzten Proben (EMAA).

\begin{tabular}{|c|c|c|c|c|c|c|c|}
\hline Probe & $\begin{array}{c}\text { Ini } \\
/ \mathrm{ml} \cdot \mathrm{min}^{-1} \\
\end{array}$ & $\begin{array}{c}\text { TxF } \\
/ \mathrm{g} \cdot(250 \mathrm{ml})^{-1}\end{array}$ & $\begin{array}{c}\text { TxB } \\
/ \mathrm{g} \cdot(250 \mathrm{ml})^{-1}\end{array}$ & $\begin{array}{c}\text { TxF } \\
/\left.10^{-2} \mathrm{~mol}^{-1}\right|^{-1} \\
\end{array}$ & $\begin{array}{c}\text { TxB } \\
/\left.\mathrm{mmol} \cdot\right|^{-1}\end{array}$ & $\begin{array}{c}\mathrm{TxF}+\mathrm{TxB} \\
/\left.10^{-2} \mathrm{~mol}^{-1}\right|^{-1}\end{array}$ & $\begin{array}{c}\text { Ini } \\
/ \mathrm{mol}^{-1} \mathrm{~h}^{-1}\end{array}$ \\
\hline EMAA 1.2 & 0.400 & 3.068 & 0.512 & 2.32 & 7.00 & 3.02 & 0.070 \\
\hline EMAA 2.2 & 0.470 & 3.095 & 0.519 & 2.34 & 7.10 & 3.05 & 0.083 \\
\hline EMAA 2.10 & 1.000 & 3.095 & 0.519 & 2.34 & 7.10 & 3.05 & 0.177 \\
\hline EMAA 3.4 & 1.650 & 3.070 & 0.510 & 2.32 & 6.98 & 3.02 & 0.289 \\
\hline EMAA 3.12 & 8.000 & 3.070 & 0.510 & 2.32 & 6.98 & 3.02 & 1.399 \\
\hline EMAA 12.11 & 0.040 & 3.073 & 0 & 4.65 & 0 & 4.65 & 0.011 \\
\hline EMAA 12.4 & 0.170 & 3.073 & 0 & 4.65 & 0 & 4.65 & 0.046 \\
\hline EMAA 16.2 & 0.380 & 3.066 & 0 & 4.64 & 0 & 4.64 & 0.102 \\
\hline EMAA 16.15 & 0.460 & 3.066 & 0 & 4.64 & 0 & 4.64 & 0.124 \\
\hline EMAA 16.5 & 0.500 & 3.066 & 0 & 4.64 & 0 & 4.64 & 0.134 \\
\hline EMAA 22.9 & 0.329 & 3.065 & 0 & 4.64 & 0 & 4.64 & 0.088 \\
\hline EMAA 22.14 & 1.290 & 3.065 & 0 & 4.64 & 0 & 4.64 & 0.346 \\
\hline
\end{tabular}




\begin{tabular}{l|ccccccc} 
Probe & $\begin{array}{c}\mathrm{Ini} \\
\mathrm{ml} \cdot \mathrm{min}^{-1} / \mathrm{g} \cdot(250 \mathrm{ml})^{-1}\end{array}$ & $\begin{array}{c}\mathrm{TxB} \\
/ \mathrm{g} \cdot(250 \mathrm{ml})^{-1}\end{array}$ & $\begin{array}{c}\mathrm{TxF} \\
/ 10^{-2} \mathrm{~mol} \cdot \mathrm{I}^{-1}\end{array}$ & $\begin{array}{c}\mathrm{TxB} \\
/ \mathrm{mmo} \cdot \mathrm{I}^{-1} / 10^{-2} \mathrm{~mol} \cdot \mathrm{I}^{-1}\end{array}$ & $\begin{array}{c}\mathrm{TxF} \\
/ \mathrm{mol} \cdot \mathrm{h}^{-1}\end{array}$ \\
\hline EMAA 25.2 & 0.280 & 3.065 & 0 & 4.64 & 0 & 4.64 & 0.075 \\
EMAA 25.6 & 0.870 & 3.065 & 0 & 4.64 & 0 & 4.64 & 0.234 \\
EMAA 27.5 & 0.172 & 3.067 & 0 & 4.64 & 0 & 4.64 & 0.046 \\
EMAA 27.8 & 0.700 & 3.067 & 0 & 4.64 & 0 & 4.64 & 0.188 \\
EMAA 28.2 & 0.280 & 0 & 1.106 & 0 & 30.26 & 3.03 & 0.049 \\
EMAA 28.6 & 1.050 & 0 & 1.106 & 0 & 15.13 & 1.51 & 0.092 \\
EMAA 28.7 & 1.700 & 0 & 1.106 & 0 & 15.13 & 1.51 & 0.149 \\
EMAA 30.3 & 0.235 & 3.061 & 0 & 4.63 & 0 & 4.63 & 0.063 \\
EMAA 31.2 & 0.237 & 3.069 & 0 & 4.64 & 0 & 4.64 & 0.064 \\
EMAA 32.2 & 0.250 & 3.061 & 0 & 4.63 & 0 & 4.63 & 0.067 \\
EMAA 32.3 & 0.570 & 3.061 & 0 & 4.63 & 0 & 4.63 & 0.153 \\
EMAA 33.3 & 0.270 & 3.061 & 0 & 4.63 & 0 & 4.63 & 0.072 \\
EMAA 33.8 & 0.890 & 3.061 & 0 & 4.63 & 0 & 4.63 & 0.239
\end{tabular}

Tabelle 7.2: Initiatorzusammensetzung und -dosierung bei der EMAACopolymerisation.

\begin{tabular}{l|ccc} 
Probe & $\begin{array}{c}M_{\mathrm{n}} \\
/ 10^{4} \mathrm{~g} \cdot \mathrm{mol}^{-1}\end{array}$ & $\begin{array}{c}M_{\mathrm{w}} \\
/ 0^{4} \mathrm{~g} \cdot \mathrm{mol}^{-1}\end{array}$ & $P D I$ \\
\hline EMAA 1.2 & 2.045 & 7.989 & 3.9 \\
EMAA 2.2 & 1.446 & 6.294 & 4.4 \\
EMAA 2.10 & 0.751 & 1.899 & 2.5 \\
EMAA 3.4 & 0.985 & 2.623 & 2.7 \\
EMAA 3.12 & 0.376 & 0.659 & 1.8 \\
EMAA 12.11 & 5.366 & 23.000 & 4.3 \\
EMAA 12.4 & 1.901 & 9.670 & 5.1 \\
EMAA 16.2 & 1.960 & 3.741 & 1.9 \\
EMAA 16.15 & 0.986 & 1.548 & 1.6 \\
EMAA 16.5 & 0.863 & 1.348 & 1.6 \\
EMAA 22.9 & 1.325 & 2.993 & 2.3 \\
EMAA 22.14 & 0.566 & 1.171 & 2.1 \\
EMAA 25.2 & 1.679 & 8.238 & 4.9 \\
EMAA 25.6 & 0.676 & 1.644 & 2.4 \\
EMAA 27.5 & 2.612 & 9.998 & 3.8 \\
EMAA 27.8 & 0.987 & 3.723 & 3.8 \\
EMAA 28.2 & 1.171 & 8.110 & 6.9 \\
EMAA 28.6 & 0.461 & 1.411 & 3.1 \\
EMAA 28.7 & 0.308 & 0.775 & 2.5 \\
EMAA 30.3 & 2.224 & 23.260 & 10.5 \\
EMAA 31.2 & 2.331 & 22.350 & 9.6 \\
EMAA 32.2 & 1.470 & 8.870 & 6.0 \\
EMAA 32.3 & 0.599 & 1.508 & 2.5 \\
EMAA 33.3 & 1.752 & 9.866 & 5.6 \\
EMAA 33.8 & 0.720 & 2.326 & 3.2
\end{tabular}

Tabelle 7.3: $\quad$ Ergebnisse der Hochtemperatur-GPC-Analyse für EMAA-Copolymere. 


\begin{tabular}{l|cccccccc} 
Probe & $\begin{array}{c}p \\
/ \text { bar }\end{array}$ & $\begin{array}{c}T_{\text {Mantel }} \\
/{ }^{\circ} \mathrm{C}\end{array}$ & $\begin{array}{c}T_{\text {Reak }} \\
/{ }^{\circ} \mathrm{C}\end{array}$ & $\begin{array}{c}\mathrm{E} \\
/ \mathrm{g} \cdot \mathrm{h}^{-1}\end{array}$ & $\begin{array}{c}\mathrm{MMA} \\
/ \mathrm{g} \cdot \mathrm{h}^{-1}\end{array}$ & $\begin{array}{c}f_{\mathrm{MMA}} \\
/ 10^{-3}\end{array}$ & $\begin{array}{c}X \\
/ \%\end{array}$ & $\begin{array}{c}F_{\mathrm{MMA}} \text { theo } \\
/ 10^{-2}\end{array}$ \\
\hline EMMA 6.27 & 2000 & 260 & $271-272$ & 1000 & 47.2 & 13.0 & 10.6 & 6.4 \\
EMMA 6.30 & 1200 & 260 & $275-276$ & 1000 & 47.2 & 13.0 & 11.6 & 6.2 \\
EMMA 8.3 & 2300 & 240 & $275-284$ & 1800 & 66.0 & 18.1 & 17.1 & 5.2 \\
EMMA 8.6 & 1300 & 240 & $265-272$ & 1800 & 66.0 & 18.1 & 11.3 & 6.7
\end{tabular}

Tabelle 7.4: $\quad$ Syntheseparameter, Umsatz und Copolymerzusammensetzung der für weitere Auswertungen benutzten Proben (EMMA).

\begin{tabular}{l|cccccccc} 
Probe & $\begin{array}{c}p \\
/ \text { bar }\end{array}$ & $\begin{array}{c}T_{\text {Mantel }} \\
/{ }^{\circ} \mathrm{C}\end{array}$ & $\begin{array}{c}T_{\text {Reak }} \\
/{ }^{\circ} \mathrm{C}\end{array}$ & $\begin{array}{c}\mathrm{E} \\
/ \mathrm{g} \cdot \mathrm{h}^{-1}\end{array}$ & $\begin{array}{c}\mathrm{AA} \\
/ \mathrm{g} \cdot \mathrm{h}^{-1}\end{array}$ & $\begin{array}{c}f_{\mathrm{AA}} \\
/ 10^{-3}\end{array}$ & $\begin{array}{c}X \\
/ \%\end{array}$ & $\begin{array}{c}F_{\text {AA }} \text { theo } \\
/ 10^{-2}\end{array}$ \\
\hline EAA 10.2 & 2300 & 220 & $264-268$ & 1800 & 21.0 & 4.5 & 21.2 & 1.9 \\
EAA 10.5 & 1300 & 220 & $258-262$ & 1800 & 21.0 & 4.5 & 19.4 & 1.9 \\
EAA 11.2 & 2300 & 220 & $256-264$ & 1800 & 84.0 & 18.0 & 19.7 & 6.7 \\
EAA 12.7 & 1300 & 220 & $264-266$ & 1800 & 84.0 & 18.0 & 25.6 & 6.2
\end{tabular}

Tabelle 7.5: $\quad$ Syntheseparameter, Umsatz und Copolymerzusammensetzung der für weitere Auswertungen benutzten Proben (EAA). 


\section{Danksagung}

Mein besonderer Dank gilt meinem Doktorvater, Prof. M. Buback, ohne den diese Dissertation nicht möglich gewesen wäre. Über die interessante Themenstellung hinaus hatte er ein offenes Ohr für wissenschaftliche und technische Probleme. Besonders hervorheben möchte ich das in mich gesetzte Vertrauen, was die tägliche Arbeit in vielerlei Hinsicht erheblich vereinfachte.

Meinen Zweitbetreuern Prof. A. Zippelius und Prof. K. Samwer danke ich für das Vorantreiben dieser Arbeit und die mir entgegengebrachte Diskussionsbereitschaft.

Herrn Dr. H. P. Vögele gilt mein Dank für die Hilfe beim Umgang mit den Spektrometern und darüber hinausgehende Beratung.

Vielen Dank für die Hilfsbereitschaft der Institutswerkstätten und der Servicekräfte.

Für die Durchführung von Analysen, bzw. für die Möglichkeit selbst tätig zu werden danke ich Dr. M. Baldus, Prof. M. Busch, Prof. H.-U. Krebs, Dr. P. Lunkenheimer, Prof. K. Samwer und ihren Arbeitsgruppen sowie M. Mänz.

Besonders herausheben möchte ich in diesem Zusammenhang die sehr fruchtbare Zusammenarbeit mit Jörg Hachenberg, der unermüdlich neue ,zylinderartige“ Proben mittels DMA untersucht hat. Thorsten Scharf und Andreas Meschede waren eine große Hilfe bei der Durchführung von PLD-Experimenten. Robert Schneider hat unaufhaltsam nach benachbarten Säuregruppen mittels NMR-Messungen Ausschau gehalten.

Die Probenherstellung wäre ohne Undrakh Nergui, Heike Rohmann und Sandra Lotze nicht möglich gewesen.

Meinen Bürokollegen Matthias Müller, Elena Müller, Undrakh Nergui, Andreas Redeker, Thomas Theis, Chunqiao Wang, Toshihiko Arita möchte ich für zahlreiche Diskussionen im wissenschaftlichen Kontext und darüber hinaus danken.

Den „Kellerkindern“ Florian Becker, Henning Latz, Stefan Hinrichs, Stephan Jauer und besonders Matthias Müller möchte ich für die Einweihung in die unerklärlichen Geheimnisse des Minitechnikums danken.

Den Korrekturlesern Moritz Gadermann, Jörg Hachenberg, Martin Mänz, Andreas Meschede, Robert Schneider und Sonja Steisel danke ich für das Auffinden von Fehlern.

Den langjährigen Studienkollegen Daniel Boschmann, Florian Lotz und Martin Mänz danke ich für Rat und Tat während der gesamten Studiendauer.

Meiner Frau Sonja Steisel danke ich für Alles. 



\section{Lebenslauf}

23.09.1980 geboren in Kassel

\section{Schulausbildung}

$1987-1991$

Grundschule Helsa

1991-1997

Valentin-Traudt-Schule, Großalmerode (Gesamtschule)

1997-2000

Freiherr-vom-Stein-Schule, Hessisch Lichtenau (Gesamtschule)

$06 / 2000$

Abitur

\section{Hochschulausbildung}

$10 / 2000-07 / 2004$

Diplomstudiengang Chemie an der Georg-August-Universität Göttingen

$10 / 2002$

Diplomvorprüfung

$12 / 2003-05 / 2004$

07/2004

$10 / 2003-09 / 2004$

Anfertigung der Diplomarbeit am Institut für Physikalische Chemie im Fachbereich Technische und Makromolekulare Chemie unter der Anleitung von Herrn Prof. Dr. M. Buback mit dem Thema: „Messung und Modellierung der radikalischen Polymerisation von Methylacrylat bis zu hohen Drücken und Temperaturen“ Diplomprüfung

Studentische Hilfskraft am Institut für Physikalische Chemie der Georg-August-Universität Göttingen

09/2004-11/2007 Stipendiat der Deutschen Forschungsgemeinschaft

seit $09 / 2004$ Anfertigung einer Dissertation am Institut für Physikalische Chemie im Fachbereich Technische und Makromolekulare Chemie unter der Anleitung von Herrn Prof. Dr. M. Buback und Herrn Prof. Dr. K. Samwer

seit $10 / 2004$ Wissenschaftliche Hilfskraft am Institut für Physikalische Chemie der Georg-August-Universität Göttingen 
